\title{
Development of bacterial nitroreductase enzymes for noninvasive imaging in cancer gene therapy
}

\author{
By \\ Elsie May Williams \\ A thesis submitted to the Victoria University of Wellington \\ in fulfilment of the requirements for the degree of \\ Doctor of Philosophy \\ in Biotechnology
}

Victoria University of Wellington

(2013) 



\begin{abstract}
There is strong interest in developing novel targeted cancer therapies. It has been known for over a century that certain viruses and bacteria can preferentially infect and lyse cancerous cells. Clinical utility has lagged behind the initial promise of the idea; however three therapeutic agents from the oncolytic virus field are currently in Phase IIB/Phase III clinical trials. The development path of such therapies would be substantially smoothed by an ability to noninvasively monitor their location in the patient's body post-administration. This would allay fears that viral/bacterial distribution may not be confined to the tumour and provide real time information on vector localisation and replication. This could be achieved by positron emission tomography (PET) scanning if the vector expressed a reporter protein which could activate a PET suitable imaging agent. Furthermore the potency of such therapies could be increased by if this reporter protein could also act therapeutically by converting a systemically delivered benign prodrug into a potent chemotherapeutic - thus targeting the toxicity of the prodrug specifically to cancerous cells. A promising enzyme/prodrug combination is the use of bacterial nitroreductase (NTR) enzymes to activate DNA damaging prodrugs, such as the dinitrobenzamides CB1954 and PR-104A.
\end{abstract}

This thesis presents work aimed at developing the ability to noninvasively image bacterial NTR expression so that these enzymes can act as both therapeutic and reporter proteins. The primary focus of this study was to achieve this by repurposing pre-existing 2-nitroimidazole (NI) PET imaging agents, originally developed for imaging tumour hypoxia. Microplate based screening strategies were developed to enable detection of 2-NI bioreductive activation by different bacterial NTRs over-expressed heterologously in Escherichia coli, and these technologies were used to screen a 58-membered library of nitroreductase candidates. Although the most widely studied NTR for enzyme/prodrug therapy - NfsB from E. coli - was found to lack activity with 2-NI substrates, numerous NTRs from the NfsA family were able to metabolise these molecules to the cell entrapped form required for PET imaging. Following this discovery, a directed evolution study was conducted to improve the native activity of the enzyme NfsA from E. coli. In this study targeted mutagenesis of active site residues was carried out, resulting in identification of several NfsA multi-site mutants that were substantially improved in their ability to activate a range of 2-NI imaging agents.

In addition to repurposing existing PET probes, this work sought to identify and engineer NTRs for efficient activation of a next-generation PET probe that is designed to be substantially less responsive to hypoxia and hence give a cleaner signal for NTR imaging (i.e. low to no background resulting from tumour hypoxia). SN33623, a novel 5-NI analogue of the existing 2-NI PET probe EF5, was designed and synthesised by our University of Auckland collaborators. It was found that this novel probe was not only activated by NfsA enzymes, but also by a subset of NfsB enzymes. Although this subset did not include E. coli $\mathrm{NfsB}$, sequence alignment and site-directed mutagenesis were used to identify two key mutations that can be introduced into $E$. coli $\mathrm{NfsB}$ (as well as engineered variants thereof) to confer high levels of SN33623 activity.

Finally work was carried out, as part of a wider collaborative project, to generate NfsA mutants that retained the ability to metabolise 2-NI imaging agents while also showing increased activation of the nitroaromatic prodrug PR-104A. Ongoing evaluation of these enzymes will include assessment of their therapeutic effect in preclinical models and their ability to be noninvasively imaged (by microPET) when expressed from the tumour targeting bacterial strain Clostridium sporogenes. 


\section{Acknowledgments}

Firstly I would like to acknowledge and thank the Tertiary Education Commission and Victoria University for funding my studies so that I could complete this $\mathrm{PhD}$ without having to be too much of a poor starving student.

I am also extremely grateful for our collaborators Adam Patterson and Jeff Smaill at the Auckland Cancer Society Research Centre, for the exciting ideas, experiments and fun new chemical compounds that they generate, with a particular thank you to Amir for synthesising so many of them. I would also like to thank them, and everyone at the ACSRC, for putting up with me bumbling my way around those fancy mammalian cells for two months, especially Alex and Chris for their help in the lab and for never making me feel like a bother with all of my tissue culture newby questions, even in the midst of their own super busyness. That I wasn't completely out of my depth is also greatly due to Laura down here at Vic, and the excellent crash course in tissue culture that she gave me.

Back in our own labs I need to give a massive thank you to Janine for both being such a lovely friend and for all the ways I've benefited in the last four years from the work you've put into the wider project and experimental advice on thorny issues. And as for the rest of you! I just want to say that over the last four years, even on days when the bacteria and the nitroretardases are misbehaving, it's still been easy to look forward to coming into work since I am lucky enough to have such an awesome group of people to share both the lab and Becky's office with and that even if nothing else gets accomplished in a day I can still take comfort in trying to beat you all at finding the nine letter word. Dave you really deserve so much credit for making our lab environment what is and I can't thank you enough for being a far more supportive, inspiring and all around better supervisor than anyone deserves - let alone me. That I wanted to keep on doing science after my honours year is really mostly all your fault.

Finally I want to thank all my other friends and my lovely family for being such a wonderful and occasionally much needed distraction from the world of science, and now that this "report" is actually handed in I am looking very much forward to actually being able to spend time with you all! I'd particularly like to thank my sister Rose for making sure I was well fed and watered/wined/beered during the last few weeks. I am also so grateful for both of my parents, whenever either of you have said that you're proud of me I really just think how proud I am to be your daughter and how grateful I am for all the help you've given me. 


\section{Table of Contents}

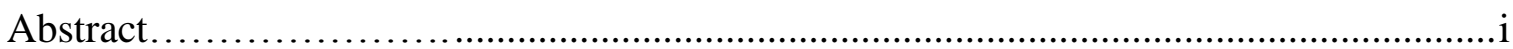

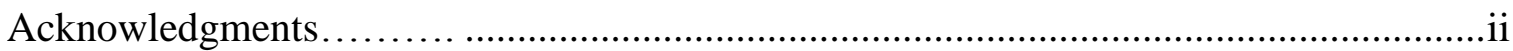

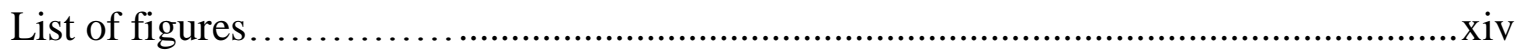

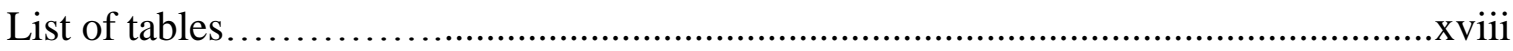

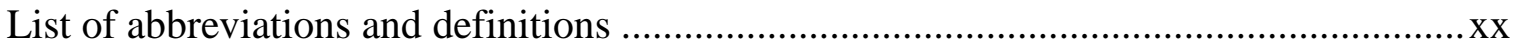

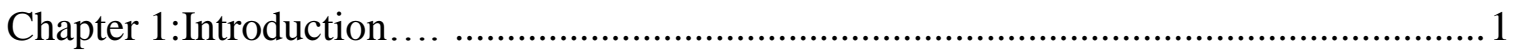

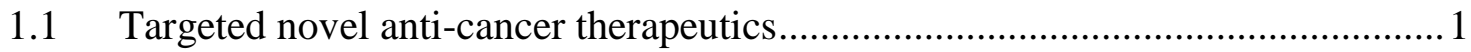

1.2 Oncolytic viruses as targeted anti-cancer therapeutics .................................. 1

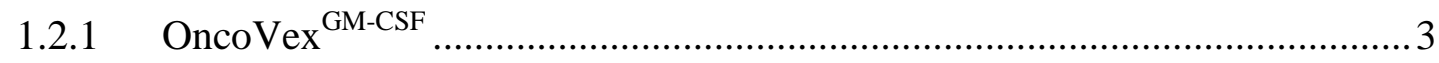

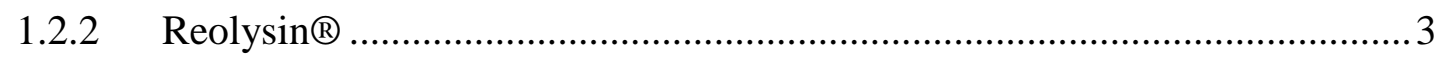

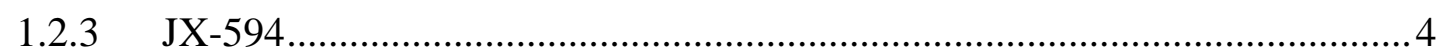

1.3 Bacteria as targeted anti-cancer therapeutics ............................................ 4

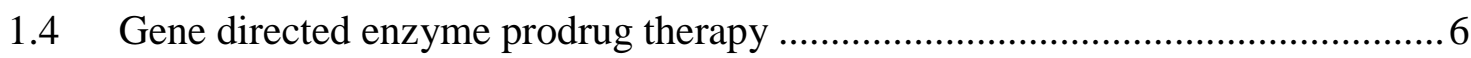

1.4.1 Herpes simplex virus thymidine kinase and ganciclovir ............................ 7

1.4.2 Bacterial nitroreductase NfsB and CB1954 _......................................... 7

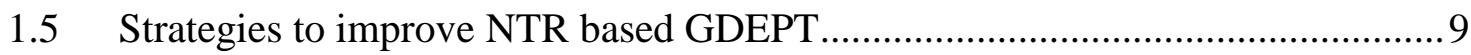

1.5.1 Alternative nitroaromatic prodrugs........................................................ 9

1.5.2 Alternative bacterial nitroreductases for GDEPT .................................... 12

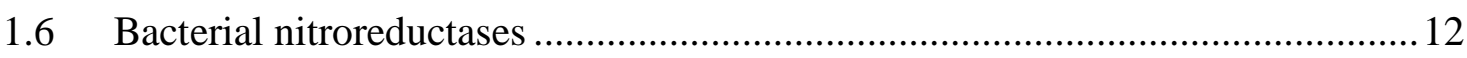

1.7 Non-invasive imaging of anti-cancer gene therapy ...................................... 14

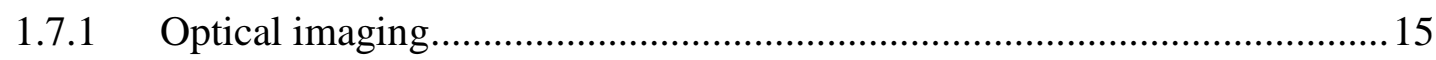

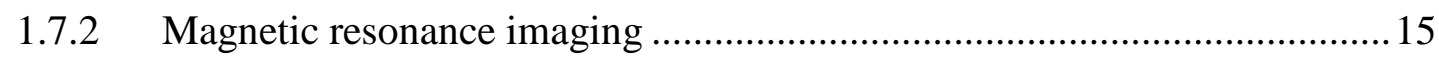

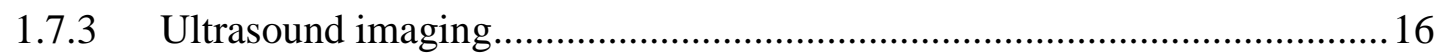

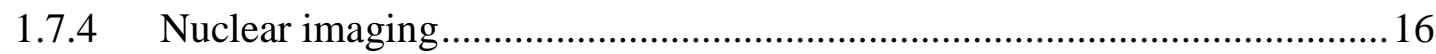

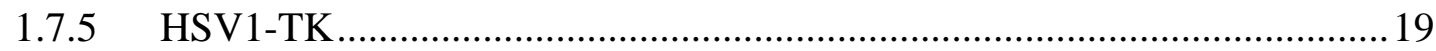

1.7.5.1 Preclinical nuclear imaging of bacterial cancer therapies ................................. 20 
1.7.6 Human sodium iodide symporter ............................................................20

1.8 Non-invasive imaging of bacterial nitroreductase enzymes.............................22

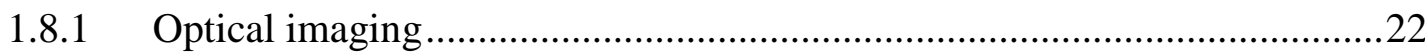

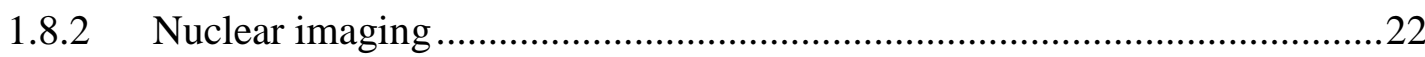

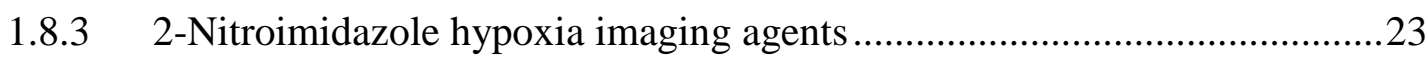

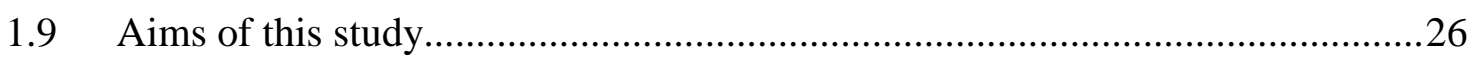

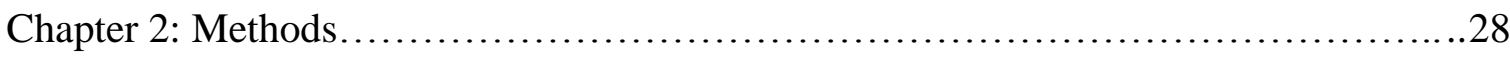

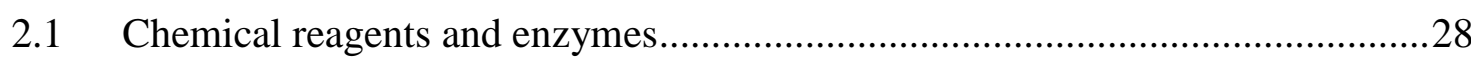

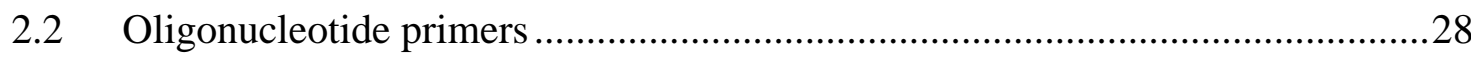

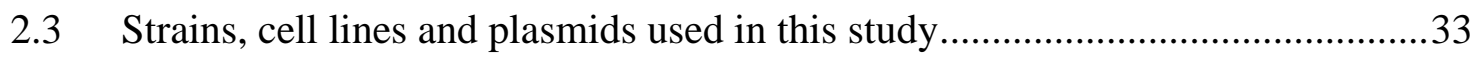

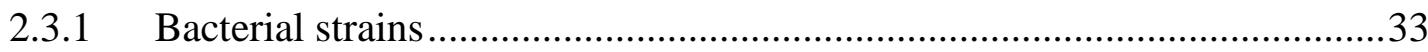

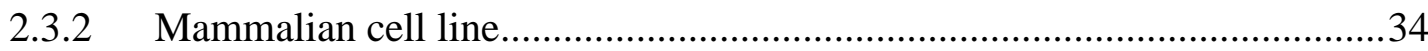

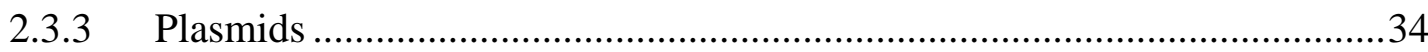

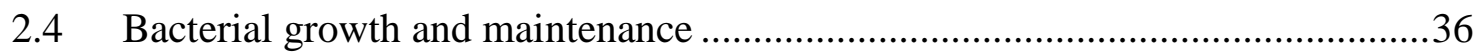

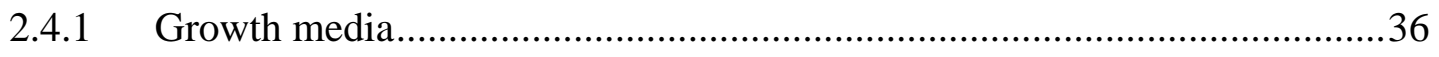

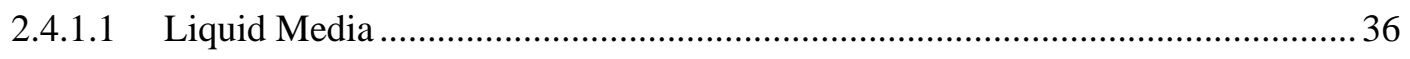

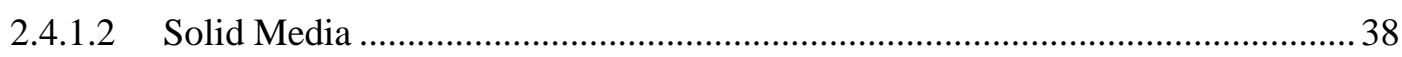

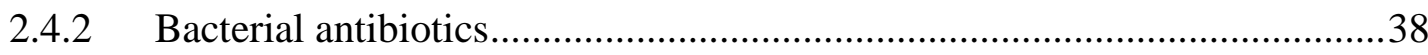

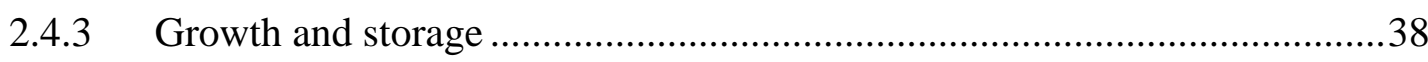

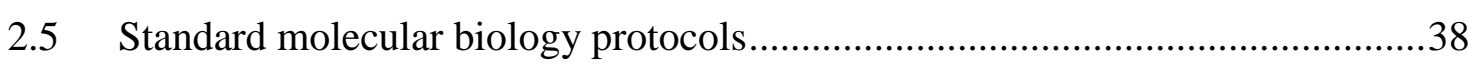

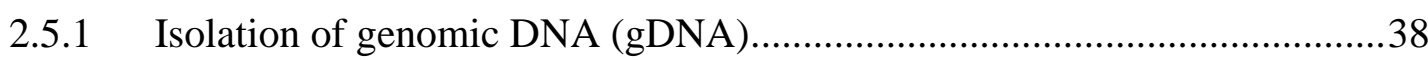

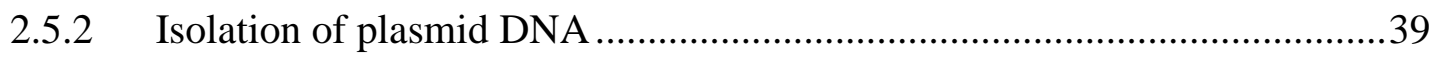

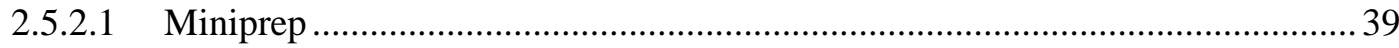

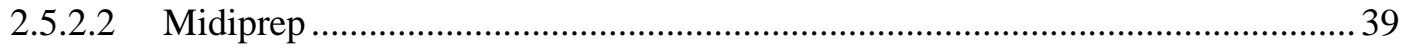

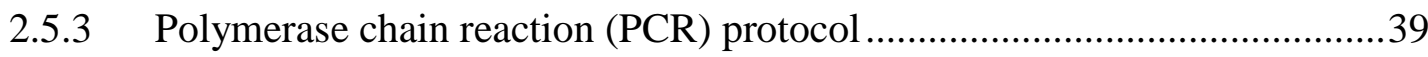

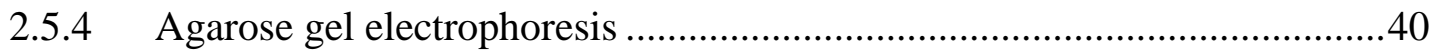

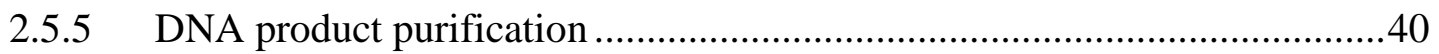

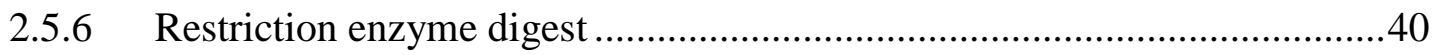


2.5.8 Cloning of high insert ratio mutant gene libraries ....................................41

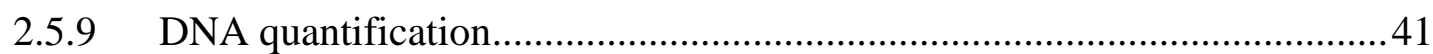

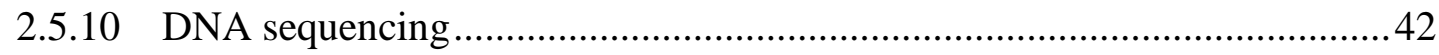

2.5.11 Transformation/electroporation of E. coli .......................................... 42

2.5.11.1 Generation of chemically competent cells ...................................................... 42

2.5.11.2 Transformation of chemically competent cells ........................................... 42

2.5.11.3 Generation of electrocompetent cells ......................................................... 43

2.5.11.4 Electroporation of electrocompetent cells ................................................. 43

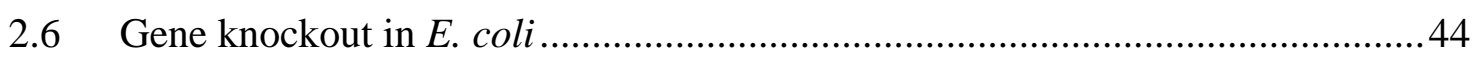

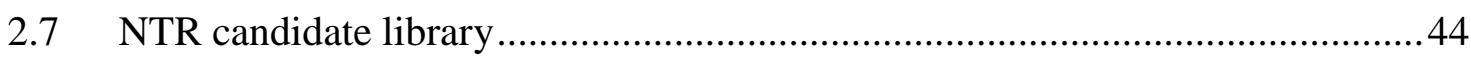

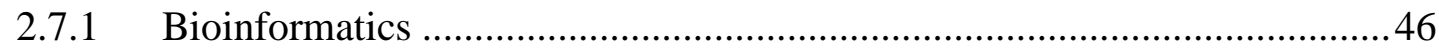

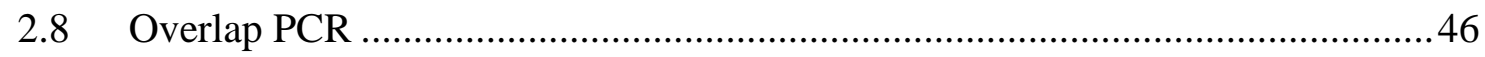

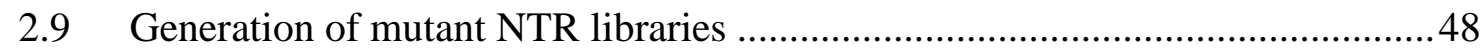

2.9.1 Modifications to electroporation protocol for the generation of mutant libraries which were to be assessed by FACS ...................................................... 49

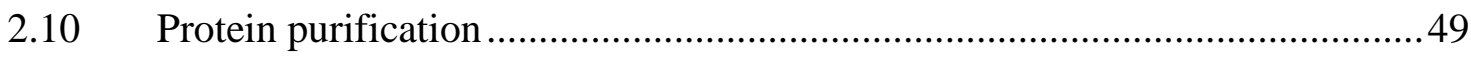

2.10.1 Expression using Autoinduction media .................................................49

2.10.2 Expression using low temperature LB + supplements..............................50

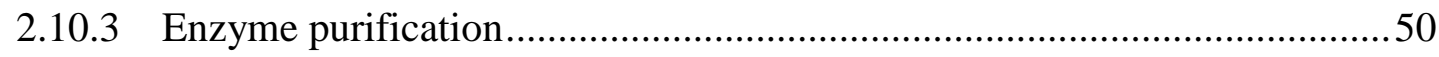

2.10.4 Determining enzyme concentration ................................................... 51

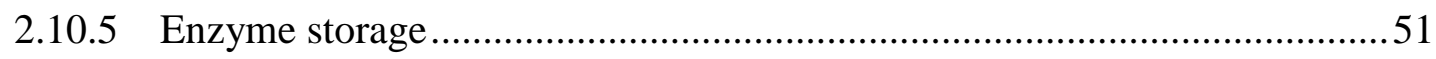

$2.11 \quad$ In vitro purified protein kinetics ......................................................... 51

2.11.1 Determination of 2-NI extinction coefficients ........................................51

2.12 Thermal scanning experiments to determine protein melting temperatures ...52

2.13 Sodium Dodecyl Sulphate Polyacrylamide Gel Electrophoresis (SDS-

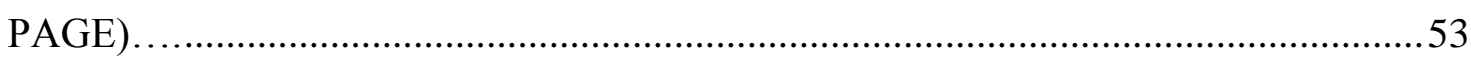

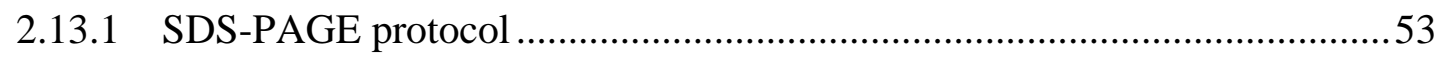




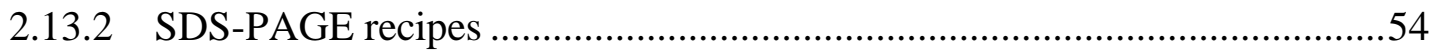

2.14 Assays for NTR activity with nitroaromatic substrates ...............................55

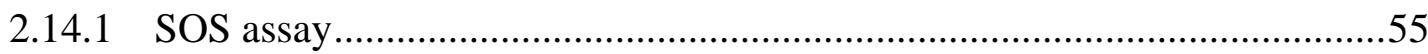

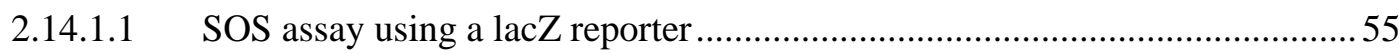

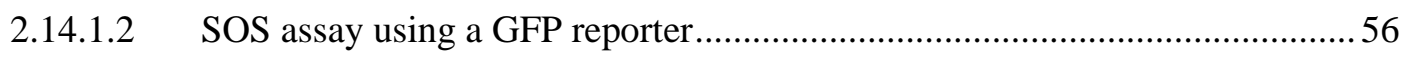

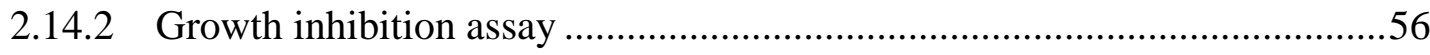

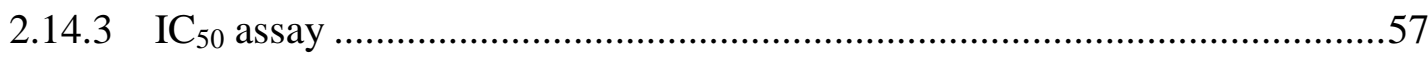

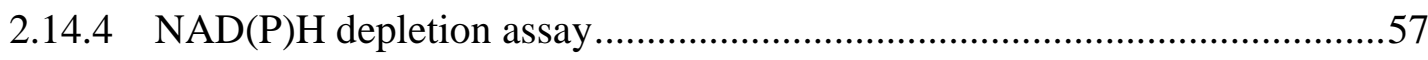

2.14.5 NTR activation of fluorogenic probes in E. coli .....................................58

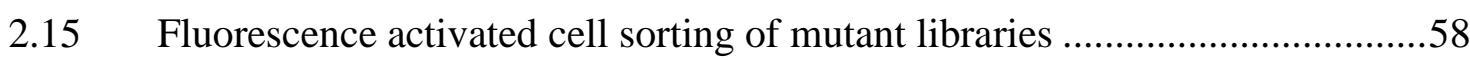

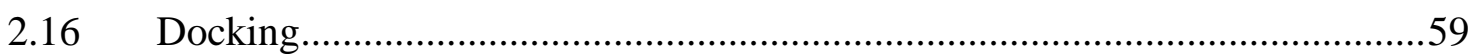

2.17 Generation, maintenance and storage of HCT-116:NTR cell lines ..................59

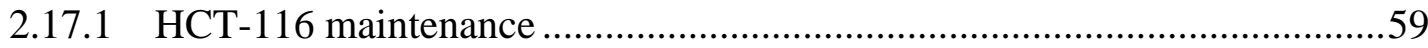

2.17.2 Storage and recovery from frozen stocks .............................................60

2.17.3 Generating F279-V5:ntr construct using Gateway ${ }^{\circledR}$ technology ................60

2.17.3.1 Generating Gateway ${ }^{\circledR}$ compatible PCR products .............................................60

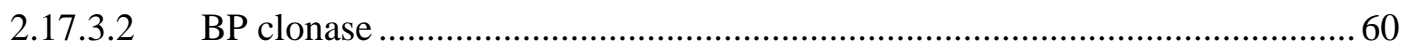

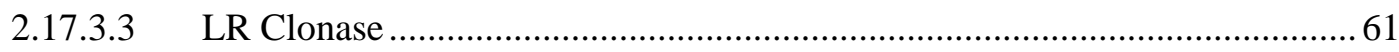

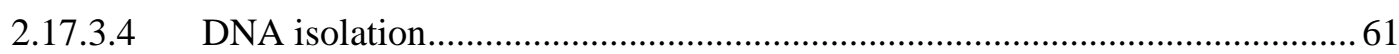

2.17.4 Transfection of HCT-116 cells ..............................................................61

2.17.4.1 FuGENE 6/X-tremeGENE HP transfection ..................................................... 61

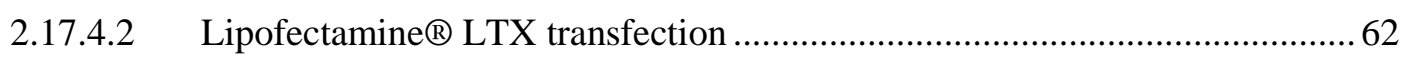

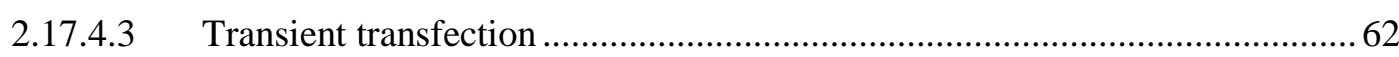

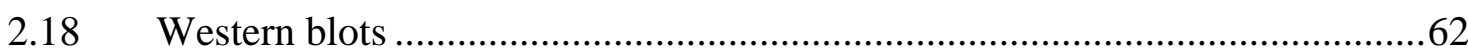

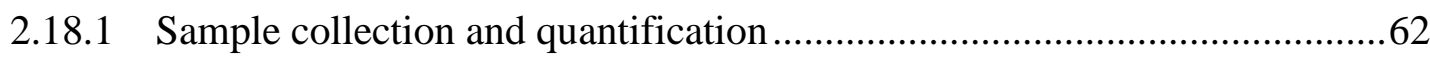

2.18.2 Gel electrophoresis separation and transfer of proteins ............................63

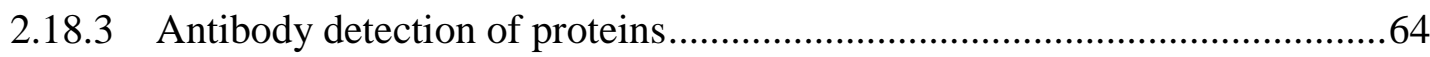

2.19 Analysis of activation of fluorogenic probes by HCT-116 cell lines ..............65

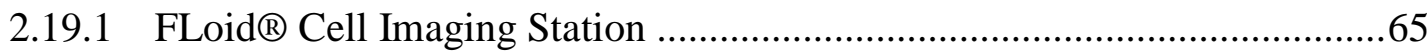


2.19.2 Flow cytometry 65

2.20 Analysis of EF5/SN33623 metabolism by HCT-116 cell lines. 65

2.21 Determining in vivo hypoxia dependant metabolism of EF5 and SN33623 ...66

2.21.1 Animal handling 66

2.21.2 Treatment of mice carrying HCT-116 tumours with imaging agents ..........66

2.21.3 Immunohistochemical staining of tumour sections .................................66

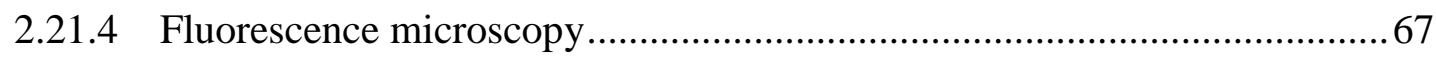

Chapter 3: Evaluation of $11 \mathrm{E}$. coli oxidoreductase enzymes for metabolism of 2-

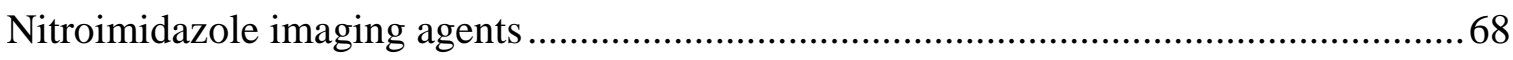

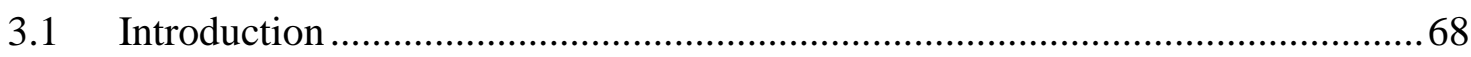

3.1.1 Identification of diverse Escherichia coli nitroreductase candidates ..........68

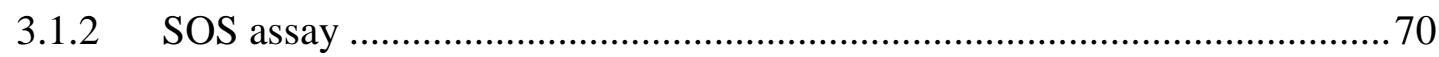

3.1.1 Nitroaromatic compounds designed for imaging tumour hypoxia ..............72

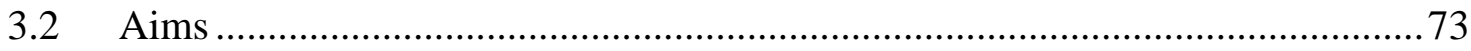

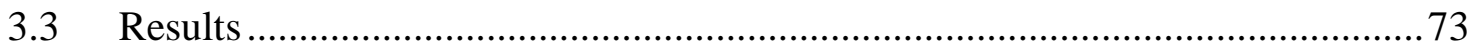

3.3.1 Evaluation of NTR activity with 2-NI imaging agents ............................. 73

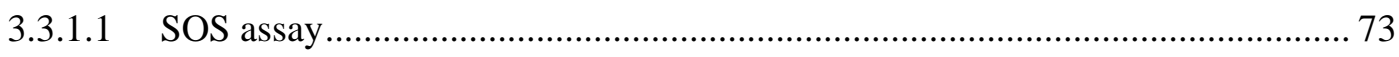

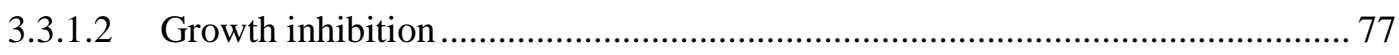

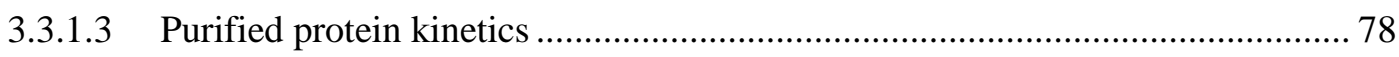

3.3.2 Optimisation of a microplate based NAD(P)H depletion assay .................. 80

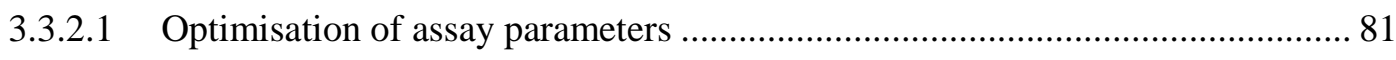

3.3.2.2 CB1954 reduction- SOS assay vs. NAD(P) H depletion assay......................... 81

3.3.3 Evaluation of NTR activity with 2-NI imaging agents using the NAD(P)H

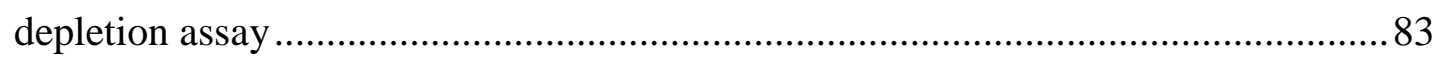

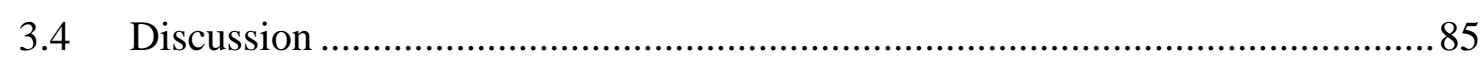

Chapter 4: Screening of an expanded bacterial oxidoreductase library for enzymes which

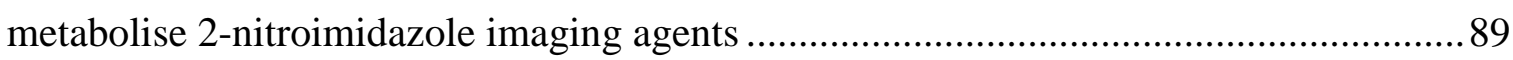

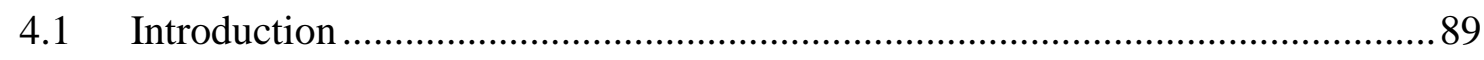

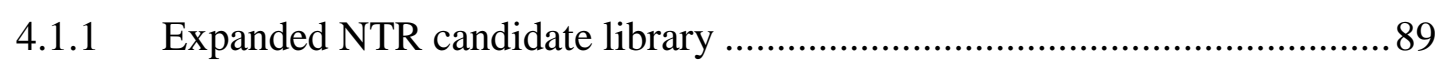




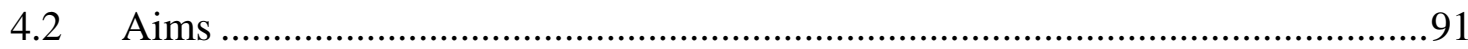

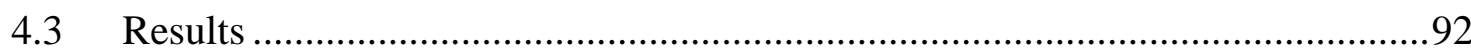

4.3.1 Screening of the expanded NTR candidate library by monitoring 2-NI

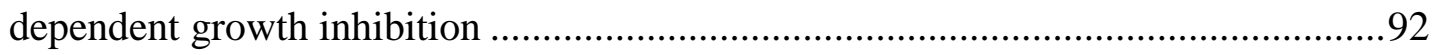

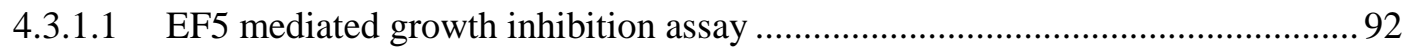

4.3.1.2 $\mathrm{IC}_{50}$ values for selected E. coli NTR over-expression strains challenged with

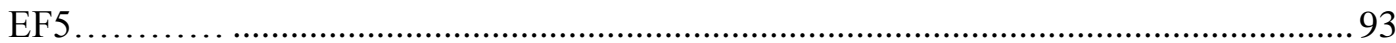

4.3.1.3 F-miso mediated growth inhibition and $\mathrm{IC}_{50}$ determination ...............................94

4.3.2 Screening of the expanded NTR candidate library using a RB6145 SOS assay 96

4.3.3 Evaluation of EF5 metabolism using the NADPH depletion assay .............98

4.3.4 Inconsistencies between the growth inhibition and NADPH depletion assays .99

4.3.5 Evaluation of EF5 reduction by purified His6-tagged NTRs .....................99

4.3.6 The ability of the kinetic parameters of purified protein reduction of EF5 to predict the results of the NADPH depletion assay and EF5 dependent growth inhibition. .101

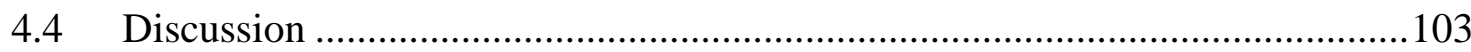

4.4.1 Screening of the expanded NTR library for 2-NI metabolism...................103

4.4.2 Expression of selected members of the expanded NTR candidate library

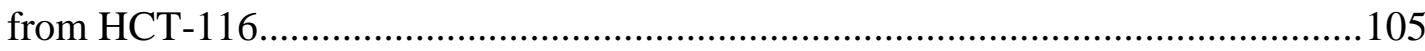

4.4.3 Conclusions regarding further E. coli based screening .............................106

Chapter 5: Directed evolution of NfsA_Ec for improved metabolism of 2-nitroimidazole

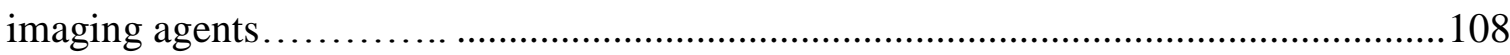

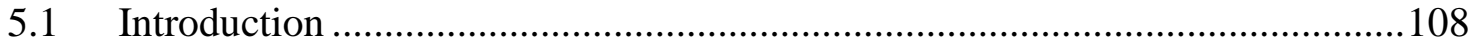

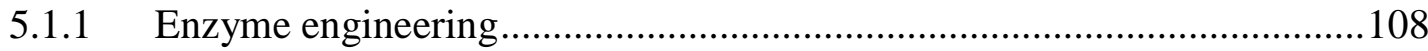

5.1.2 Docking of EF5 into the active site of NfsA_Ec ....................................110

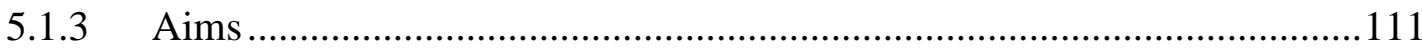

5.2 Results I: Directed evolution of NfsA_Ec using targeted site saturation mutagenesis..... 
5.2.1 Construction of targeted single site mutagenesis libraries

5.2.2 Screening of 12 site saturation mutagenesis libraries for single residue mutations conferring increased 2-NI metabolism

5.2.3 Combination of single mutations to generate NfsA_Ec variants further improved in 2-NI metabolism.

5.2.4 Further assessment of selected beneficial single mutations.

5.2.4.1 SDS-PAGE of relative protein expression 115

5.2.4.2 Reduction of EF5 by His6-tagged purified proteins 116

5.2.5 Construction of a multi-site mutant NfsA_Ec library..... 117

5.2.6 Screening of a multi-site mutant NfsA_Ec library for variants with improved 2-NI imaging agent metabolism

5.2.7 Further assessment of selected multi-site NfsA_Ec mutants.

5.2.7.1 SDS-PAGE of relative protein expression .................................................. 120

5.2.7.2 Reduction of EF5 by His6-tagged purified proteins 121

5.2.7.3 Activity with the nitroaromatic prodrug PR104-A and the 5-NI antibiotic metronidazole

5.2.8 Activity of previously identified mutants with the novel 2-NI imaging agent HX4. 122

5.2.9 Assessment of NfsA_Ec mutants showing improved 2-NI metabolism by expression in a human cancer cell line

5.2.10 Western blot of NfsA_Ec wild type and mutant expression from HCT116. 124

5.2.11 Testing for functional NTR expression using NTR activated fluorogenic probes

5.2.12 Metabolism of EF5 by HCT-116: NTR cell lines. 126

5.3 Results II: Preliminary work investigating a high-throughput screen for identification of mutants with 2-NI improved metabolism 128

5.3.1 A fluorescence activated cell sorting (FACS) based screening strategy ... 128

5.3.1.1 Large NfsA_Ec mutant library fully randomised (NNK codon) at four amino acid sites. .

5.3.1.2 FACS selection of GFP expressing cells post RB6145 challenge 129 
5.3.1.3 Screening the 2-NI metabolism of FACS selected clones................................ 130

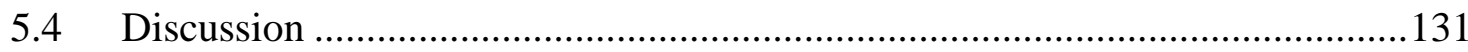

5.4.1 Generation of NfsA_Ec variants which show improved 2-NI metabolism on

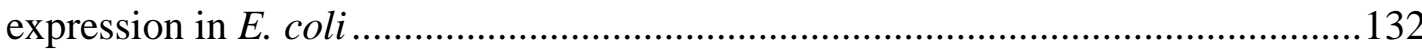

5.4.1.1 Fluorescent activated cell sorting of mutant libraries to identify 2-NI active

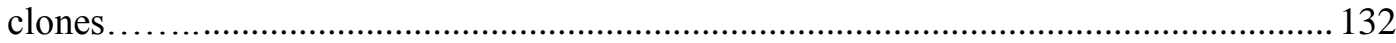

5.4.2 Models of multi-site mutants improved in 2-NI metabolism.....................133

5.4.3 Apparent kinetic parameters of NfsA_Ec variants ...................................134

5.4.4 Expression of NfsA_Ec variants from HCT-116 cells..............................135

5.4.5 Further testing of the 2-NI metabolism NfsA_Ec variants ........................136

Chapter 6: Identification and engineering of nitroreductases that activate a hypoxia

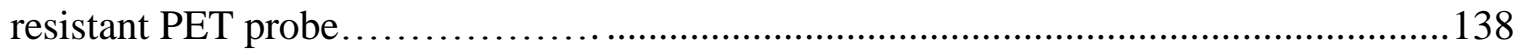

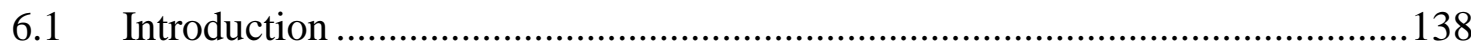

6.1.1 Design of a de novo NTR imaging agent with decreased hypoxic

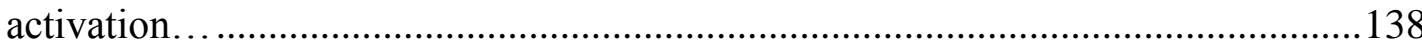

6.1.2 Reduction of SN33623 by bacterial NTRs ..........................................139

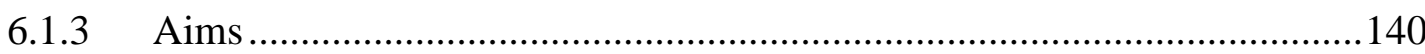

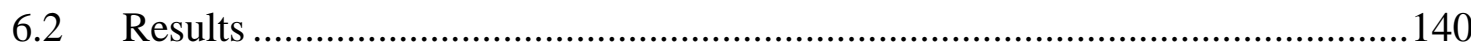

6.2.1 In vivo hypoxia dependent metabolism of SN33623 ..............................140

6.2.2 Screening of the expanded NTR candidate library with SN33623 ............142

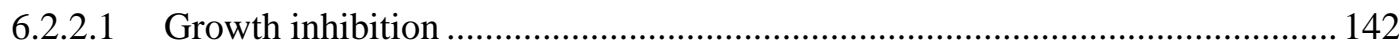

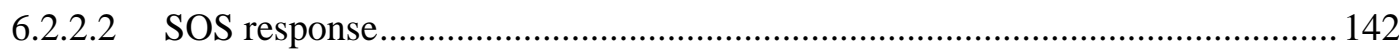

6.2.3 SN33623 metabolism and evolutionary relationships of NfsB family enzymes........................................................... 143

6.2.4 Identification of amino acid residues important in SN33623 activation ...144

6.2.5 Identification of residues playing a key role in SN33623 metabolism in

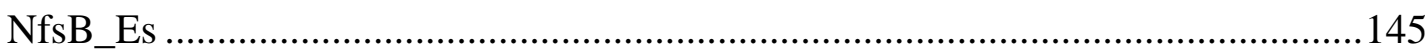

6.2.6 Specificity of the decrease in SN33623 metabolism ...............................146

6.2.7 Generation of NfsB_Ec mutants that can metabolise SN33623 ...............147 
6.2.8 Metabolism of 2-NI imaging agents by NfsB_Ec mutants engineered for

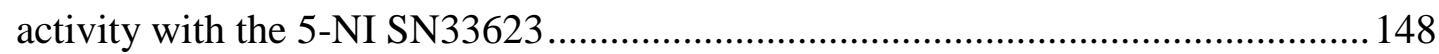

6.2.9 Metabolism of SN33623 by NfsB_Ec mutants evolved for superior CB1954 activation

6.2.10 Reduction of SN33623 by His6-tagged purified proteins.

6.2.11 Docking of SN33623 and CB1954 into the active mutant NfsB_Es F70A.

6.2.12 Testing of bacterial NTR activity when expressed by the human cancer cell line HCT-116 155

6.2.13 SN33623 metabolism by stable expression HCT-116:ntr cell lines 156

6.2.14 Western blot analysis of NTR expression from stable HCT-116:ntr cell lines 159

6.2.15 Metabolism of the NTR activated fluorogenic probe FSL61 by HCT116 159

6.2.16 Transient expression and exposure to SN33623 160

6.2.17 Thermostability 163

6.3 Discussion 164

6.3.1 Further investigation of hypoxia resistance and NTR labelling 164

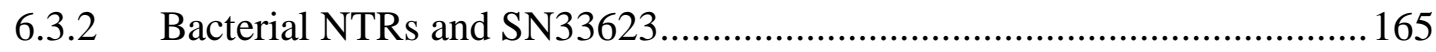

6.3.3 Expression of NfsB_Ec mutants from HCT-116 ..................................... 166

Chapter 7: Directed evolution of NfsA_Ec variants with improved prodrug activation 168

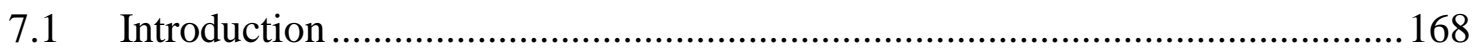

7.2 Clostridium directed enzyme prodrug therapy........................................... 168

7.2.1 Translation of nitroreductase armed Clostridium to a clinical setting....... 169

7.2.2 Evolution of NfsA_Ec for improved PR-104A activation........................ 170

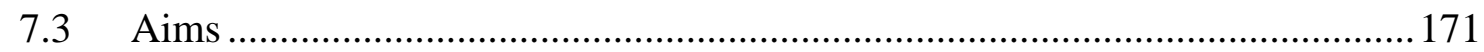

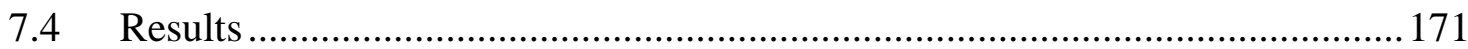


7.4.1 Evaluation of the activity of wild type NTRs with HX4 _........................171

7.4.2 Metabolism of 2-NI imaging agents by NfsA_Ec single mutants showing improved activity with PR-104A ....................................................................... 173

7.4.3 Screening of a multi site NfsA_Ec gene library for mutants exhibiting

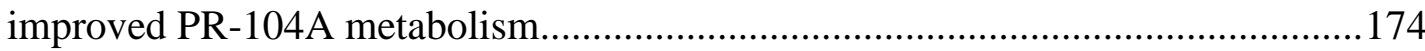

7.4.4 Reduction of PR-104A by His6-tagged purified proteins..........................176

7.4.5 2-NI metabolism of multi site NfsA_Ec mutants selected for improved PR104A activity

7.4.6 Metabolism of CB1954 and metronidazole by multi site NfsA_Ec mutants selected for improved PR-104A activity ............................................................. 178

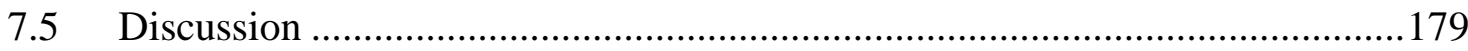

7.5.1 Selection of the NfsA_Ec variants to be introduced into C. sporogenes...180

7.5.2 MicroPET imaging of Clostridium expressing NfsA_Ec variants..............180

7.5.3 Comparison of the NfsA_Ec variants abilities to metabolise PR-104A and CB1954.. 181

Chapter 8: Key findings, conclusions and future directions ......................................183

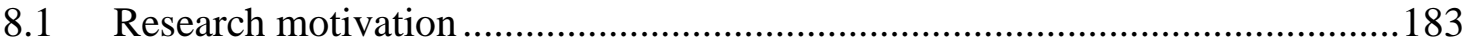

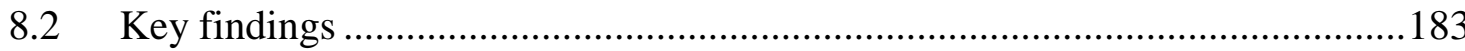

8.3 Critical evaluation of the E. coli screens used in this study ...........................185

8.3.1 Variations in bacterial NTR expression levels from human cells..............186

8.3.2 Purified protein activities of evolved NTR variants ................................187

8.3.2.1 Additional points to consider in assessment of the kinetic assays ................... 189

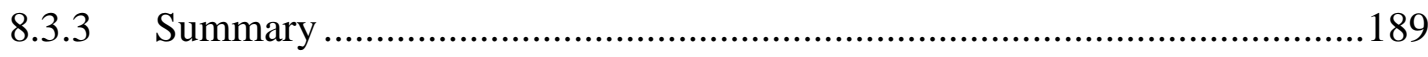

8.4 Further possible directed evolution studies ...................................................190

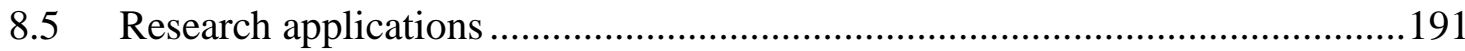

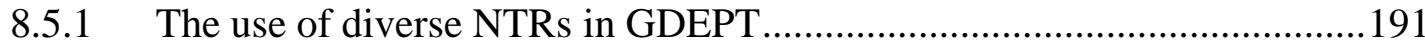

8.5.2 Noninvasive PET imaging of NTR based therapy ..................................192

8.5.3 The potential use of bacterial NTRs as vector reporter genes independent of GDEPT. . 


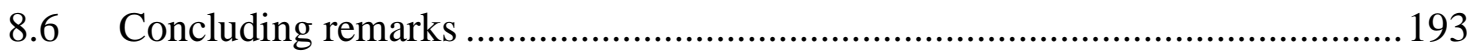

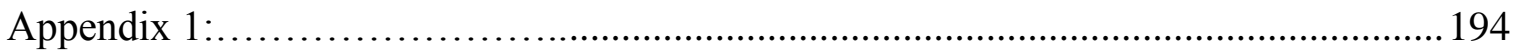

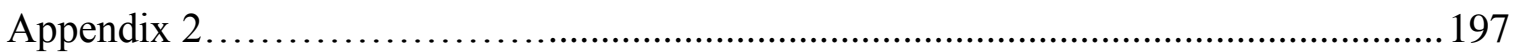

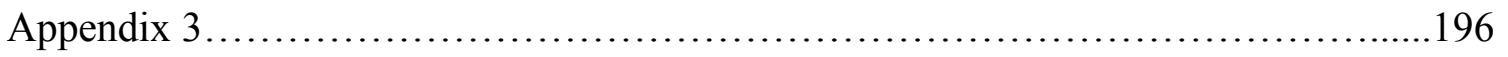

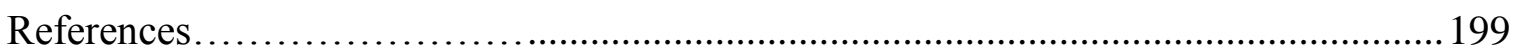




\section{List of figures}

Figure 1-1 Schematic showing principle of gene directed enzyme prodrug therapy...........6

Figure 1-2 CB1954 reduction by rat NQO1 .............................................................

Figure 1-3 Chemical structure of the dinitrobenzamide mustard PR-104 _.......................9

Figure 1-4 DNA cross-linking by a nitrogen mustard, .............................................

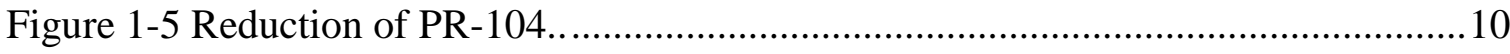

Figure 1-6 Schematic of hypoxia dependent and independent nitroaromatic reduction,...11

Figure 1-7 Schematic showing the nuclear imaging of a reporter gene/probe. ................. 17

Figure 1-8 Structures of ${ }^{18} \mathrm{~F}$ 2-NI hypoxia imaging agents with prior human use.............25

Figure 2-1 Plasmid map and multiple cloning site of E. coli expression vector pUCX

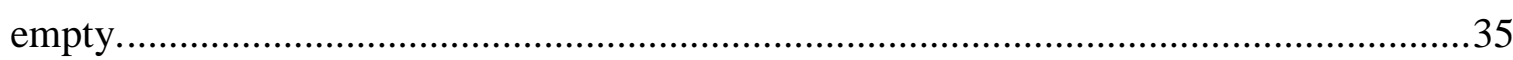

Figure 2-2 Plasmid map of F279-V5, the plasmid vector used for NTR expression from

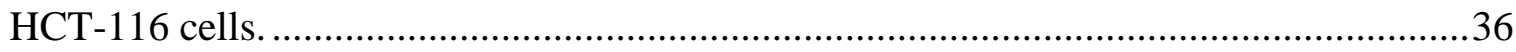

Figure 2-3 Example of primers used for generating site directed mutants .......................47

Figure 2-4 Schematic of overlap PCR for generation of site directed mutants .................47

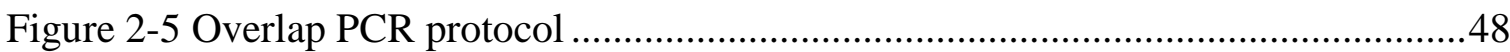

Figure 3-1 Schematic of the SOS assay used to monitor NTR activity with nitroaromatic

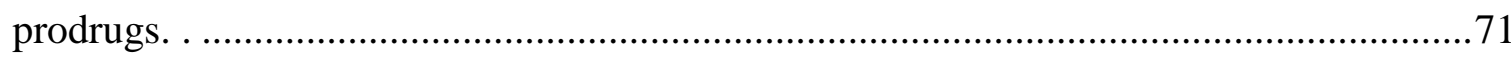

Figure 3-2 CB1954-induced SOS response induction of NTR over-expressing SOS-R1

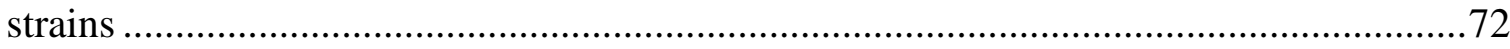

Figure 3-3 Chemical structures of 2-NI hypoxia imaging agents available to this study..73 Figure 3-4 E. coli NTR over-expression library exposed to titrated concentrations of EF5 .75

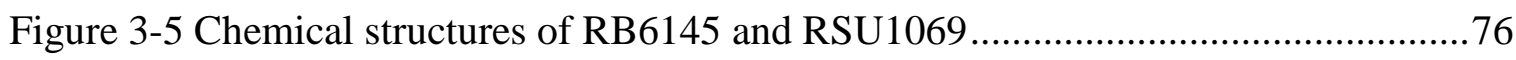

Figure 3-6 RB6145 induced SOS response of the E. coli NTR over-expression library ..77 Figure 3-7 $\mathrm{IC}_{50 \text { s }}$ of 2-NI compounds for the SOS-R2 E. coli NTR over-expression library .78

Figure 3-8 NAD(P)H depletion assay. 81

Figure 3-9 $\mathrm{NAD}(\mathrm{P}) \mathrm{H}$ consumption by SOS-R1:ntr cell lysates in the presence of CB1954.

Figure 3-10 NADPH consumption by SOS-R2:ntr cell lysates in the presence of 2-NI compounds .84 
Figure 3-11 In vivo binding of EF5 in HCT-116:ntr tumour xenografts 87

Figure 3-12 Comparison of in vivo PR-104A tumour cell kill and EF5 binding. .88

Figure 4-1 Phylogeny tree of the 58 oxidoreductases in the expanded NTR candidate library

Figure 4-2 EF5 mediated growth inhibition of SOS-R2 E.coli NTR over-expression strains .93

Figure 4-3 EF5 IC 50 values for SOS-R2 E. coli NTR over-expression strains. .94

Figure 4-4 F-miso mediated growth inhibition of SOS-R2 E.coli NTR over-expression strains.

Figure 4-5 F-miso $\mathrm{IC}_{50}$ values for SOS-R2 E. coli NTR over-expression strains. 96 Figure 4-6 RB6145 mediated induction of the SOS response E. coli SOS-R2 NTR overexpression strains

Figure 4-7 Linear correlation between the SOS response induced by RB6145 and the growth inhibitory effects of EF5 and F-miso in E. coli SOS-R2 NTR over-expression cultures.

Figure 4-8 NADPH consumption by SOS-R2:ntr cell lysates in the presence of EF5....98 Figure 4-9 Linear correlations between kinetic parameters of NTR catalysed EF5 reduction and EF5 reduction as determined by $\mathrm{A}$ ) the NADPH depletion assay $\mathrm{B}) \mathrm{IC}_{50}$ values. 102

Figure 4-10 Expression and functional validation of top nitroreductase candidates in a human cancer cell line model.

Figure 5-1 Docking of EF5 into the active site of solved crystal structure of NfsA_Ec.110 Figure 5-2 SDS-PAGE gel showing NfsA_Ec single mutant relative protein expression levels 116

Figure 5-3 Kinetic parameters for the reduction of EF5 by purified (His6-tagged) NfsA_Ec single mutants.

Figure 5-4 Representation of a multi-site mutant NfsA_Ec gene library

Figure 5-5 A) EF5 and B) F-miso $\mathrm{IC}_{50}$ values for SOS-R2 E. coli strains over-expressing NfsA_Ec wild type or mutants evolved for improved 2-NI metabolism.

Figure 5-6 SDS-PAGE gel showing NfsA_Ec relative protein expression levels of multisite mutants

Figure 5-7 PR-104A and metronidazole (Metro) $\mathrm{IC}_{50}$ values for SOS-R2 E. coli strains over-expressing NfsA_Ec wild type or mutants evolved for improved 2-NI metabolism.. 
Figure 5-8 HX4 IC $_{50}$ values for SOS-R2 E. coli strains over-expressing NfsA_Ec wild type or mutants evolved for improved 2-NI metabolism.

Figure 5-9 Western blot showing expression of NfsA_Ec wild type and mutant proteins from stable HCT-116:NTR cell lines.

Figure 5-10 Activation of three different NTR specific fluorogenic probes by HCT-116 cells expressing NfsA_Ec wild type and mutant proteins. 126

Figure 5-11 Metabolism of EF5 by stable HCT-116:NTR expression cell lines.

Figure 5-12 Schematic of the GFP-SOS assay designed for the screening of large mutant libraries using FACS

Figure 5-13 Mutations and 2-NI metabolism of NfsA_Ec mutants recovered from a FACS sort of an NfsA_Ec 414583134 NNK mutant library. 131

Figure 5-14 Possible effects of S41Y L103V N134A mutations on predicted EF5 binding modes.

Figure 6-1 Chemical structures of EF5 and the de novo potential NTR imaging agent SN33623.

Figure 6-2 Hypoxia dependent binding of A) EF5 and B) SN33623 in a mouse HCT-116 tumour xenograft model 141

Figure 6-3 SN33623 mediated growth inhibition of SOS-R2 E. coli NTR over-expression strains. 142

Figure 6-4 SN33623 mediated induction of the SOS response E. coli SOS-R2 NTR overexpression strains. 143

Figure 6-5 Relationships between NfsB family enzymes 144

Figure 6-6 Identification of amino acid residues important in SN33623 activation .145 Figure 6-7 SN33623 induced growth inhibition of E. coli SOS-R2 NfsB_Es mutant overexpression strains. .146

Figure 6-8 Metabolism of SN33623, PR-104A, Nitrofurazone (NFZ) or FSL41 by SOSR-2 E. coli strains over-expressing NfsB_Es mutants 147

Figure 6-9 SN33623 mediated growth inhibition of E. coli SOS-R2 over-expressing engineered NfsB_Ec variants. 148

Figure 6-10 A)EF5 and B)HX4 mediated growth inhibition of E. coli SOS-R2 overexpressing NfsB_Ec mutants engineered for improved SN33623 metabolism 149

Figure 6-11 SN33623 and CB1954 mediated growth inhibition of E. coli SOS-R2 overexpressing engineered $\mathrm{NfsB}$ _Ec mutants. 150 Figure 6-12 Stereo view of CB1954 docked into the NfsB_Ec F70A model. 153 
Figure 6-13 Docked structures of SN33623 in NfsB_Ec F70A model. 155

Figure 6-14 Metabolism of SN33623 by stable HCT-116:ntr expression cell lines 158

Figure 6-15 Western blot showing expression of NfsB_Ec wild type and mutant proteins from stable HCT-116:ntr cell lines.

Figure 6-16 Metabolism of the fluorogenic probe FSL61 by stably transfected HCT-116 NTR cell lines 160

Figure 6-17 Metabolism of A) FSL61 and B) SN33623 by stable and transient HCT-116 NTR expression cell lines. 162

Figure 7-1 HX4 mediated growth inhibition of SOS-R2 E. coli NTR over-expression strains.

Figure 7-2 Kinetic parameters for the reduction of HX by purified His6-tagged NTRs 173 Figure 7-3 2-NI IC 50 values of SOS-R2 E. coli strains over-expressing NfsA_Ec wild type or single mutants evolved for improved PR-104A metabolism. 174 Figure 7-4 PR-104A IC I0 $_{50}$ values of SOS-R2 E. coli strains over-expressing mutants identified as showing improved PR-104A activation 175 Figure 7-5 2-NI IC $_{50}$ values of SOS-R2 E. coli strains over-expressing NfsA_Ec wild type or mutants evolved for improved PR-104A metabolism. 177

Figure 7-6 A) CB1954 and B) metronidazole $\mathrm{IC}_{50}$ values of SOS-R2 E. coli strains overexpressing NfsA_Ec wild type or mutants evolved for improved PR-104A metabolism.

Figure 7-7 Heat map representation of activities of NfsA_Ec multi site mutants 181 
xviii

\section{List of tables}

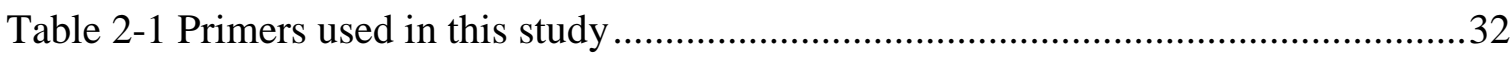

Table 2-2 Escherichia coli strains used in the study........................................................3

Table 2-3 Strains used as sources of oxidoreductases in expanded NTR candidate library .33

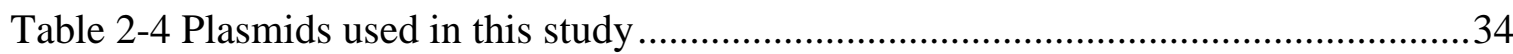

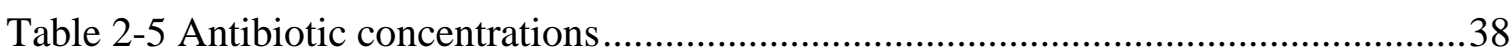

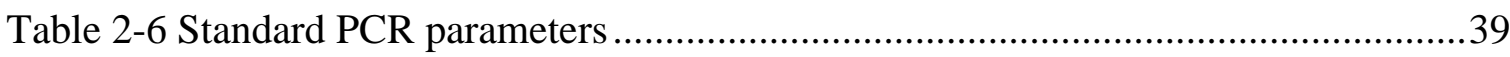

Table 2-7 Components of Phusion ${ }^{\mathrm{TM}}$ and Biomix ${ }^{\mathrm{TM}}$ Red PCR reactions...........................40

Table 2-8 Ligation components ................................................................................

Table 2-9 58 oxidoreductase enzymes in the expanded NTR candidate library,..............46

Table 2-10 Experimentally derived extinction coefficients for 2-NI compounds used in

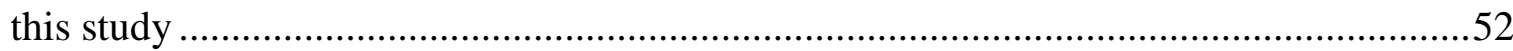

Table 2-11 Antibody dilutions for use in western blots...................................................64

Table 3-1 Kinetic parameters for the reduction of 2-NI compounds by purified His6-

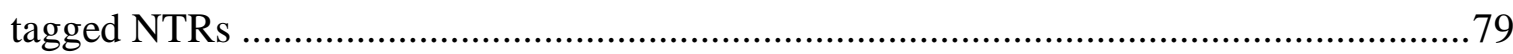

Table 4-1 Kinetic parameters for the reduction of EF5 by purified His6-tagged NTRs. 100 Table 5-1 NfsA_Ec single mutants showing increased EF5 and F-miso metabolism on over-expression in E. coli SOS-R2 . .....................................................................113

Table 5-2 Table showing IUB degenerate base code....................................................114

Table 5-3 Amino acid residue changes found in NfsA_Ec mutants exhibiting improved

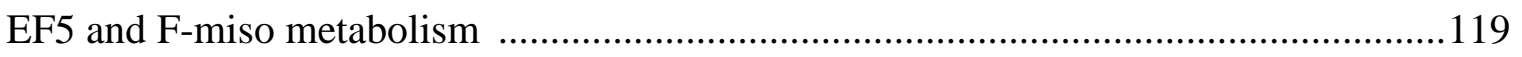

Table 5-4 Kinetic parameters for the reduction of EF5 by purified (His6-tagged) NfsA_Ec

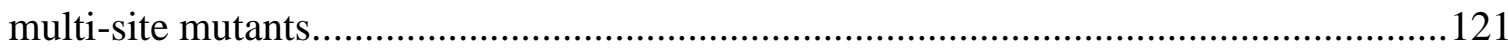

Table 6-1 NADPH consumption rate in the presence of SN33623 by purified His6-tagged NTRs.

Table 6-2 Bacterial NTRs selected for testing of SN33623 metabolism on expression from the human cancer cell line HCT-116..................................................................156

Table 6-3 Melting temperatures $\left(\mathrm{T}_{\mathrm{m}}\right)$ of His6-tagged purified NfsB_Ec and NfsB_Ec

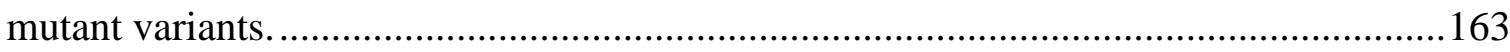

Table 7-1 Single mutations to NfsA_Ec determined by Dr Janine Copp to confer improved PR-104A metabolism.....................................................................................171 
Table 7-2 Amino acid residue changes found in NfsA_Ec mutants exhibiting improved PR-104A metabolism......

Table 7-3 Kinetic parameters of reduction of PR-104A by purified His6-tagged NTRs 176 Table A2-1 All possible degenerate codons which designated only the NfsA_Ec wild type amino acid residue or the indicated mutant residue. 195

Table A3-1Table of mutations found in random clones from NfsA_Ec mutant library.... 196

Table A3-2 Table of mutations found in random clones from NfsA_Ec mutant library with NNK codons. .196 


\section{List of abbreviations and definitions}

\begin{tabular}{|c|c|}
\hline 2-NI & 2-Nitroimidazole \\
\hline $5-\mathrm{NI}$ & 5-Nitroimidazole \\
\hline ACSRC & Auckland Cancer Society Research Centre \\
\hline Amp & Ampicillin \\
\hline BCA & Bicinchoninic Acid \\
\hline BLAST & Basic Local Alignment Search Tool \\
\hline BSA & Bovine Serum Albumin \\
\hline $\mathrm{CD}$ & Cytosine Deaminase \\
\hline Chl & Chloramphenicol \\
\hline CNOB & 6-chloro-9-nitro-5-oxo-5H-benzo \\
\hline CT & Computed tomography \\
\hline DMEM & Dulbeccos modified eagle medium \\
\hline DMSO & Dimethylsulfoxide \\
\hline e- & Electron \\
\hline EDTA & Ethylenediaminetetraacetic acid \\
\hline FACS & Fluorescence-activated cell sorting \\
\hline FAD & Flavin adenine dinucleotide \\
\hline FCS & Fetal calf serum \\
\hline F-miso & Fluoromisonidazole \\
\hline FRT & Flippase recognition target \\
\hline FMN & Flavin mononucleotide \\
\hline GCV & Ganciclovir \\
\hline GDEPT & Gene directed enzyme prodrug therapy \\
\hline GFP & Green fluorescent protein \\
\hline GM-CSF & Granulocyte macrophage colony stimulating factor \\
\hline HSV1-TK & Herpes simplex virus thymidine kinase \\
\hline $\mathrm{IC}_{50}$ & Concentration of drug required to inhibit normal growth of organism by $50 \%$ \\
\hline Kan & Kanamycin \\
\hline$k_{\text {cat }}$ & $\begin{array}{l}\text { Kinetic constant describing theoretical maximum rate of catalysis for a specific } \\
\text { enzyme with a specific substrate, under the conditions tested. }\end{array}$ \\
\hline$K_{m}$ & $\begin{array}{l}\text { Kinetic constant describing the concentration of substrate at which rate of } \\
\text { catalysis of a specific enzyme is exactly half of the Kcat, under the conditions tested. }\end{array}$ \\
\hline LB & Luria broth \\
\hline MCS & Multi cloning site \\
\hline MRI & Magnetic resonance imaging \\
\hline MTZ & Metronidazole \\
\hline NADH & Nicotinamide adenine dinucleotide (reduced) \\
\hline NADPH & Nicotinamide adenine dinucleotide phosphate (reduced) \\
\hline NCBI & National Centre for Biotechnology Information \\
\hline $\mathrm{NH}_{2}$ & Amino derivative \\
\hline $\mathrm{NHOH}$ & Hydroxylamino derivative \\
\hline
\end{tabular}




$\begin{array}{ll}\mathrm{NO}_{2} & \text { Nitro group } \\ \text { NTR } & \text { Nitroreductase } \\ \text { NQO1 } & \text { NAD(P)H:Quinone oxidoreductase 1 } \\ \text { OD }_{\text {xxx }} & \text { Optical density at xxx wavelength } \\ \text { ONPG } & \text { ortho } \text {-Nitrophenyl- } \beta \text {-galactoside } \\ \text { OYE } & \text { Old yellow enzyme } \\ \text { PCR } & \text { Polymerase chain reaction } \\ \text { NBT } & \text { Nitroblue tetrazolium chloride } \\ \text { PET } & \text { Positron emission tomography } \\ \text { PMS } & \text { Phenazine methosulfate } \\ \text { RPM } & \text { Revolutions per minute } \\ \text { SDS } & \text { Sodium dodecyl sulfate } \\ \text { SEM } & \text { Standard error of the mean } \\ \text { SPECT } & \text { Single photon emission computed tomography } \\ \text { Spec } & \text { Spectinomycin } \\ \text { TE } & \text { Tris-EDTA } \\ \text { TNT } & \text { trinitrotoluene } \\ \text { Tris } & \text { Tris(hydroxymethyl)aminomethane } \\ \text { v/v } & \text { Volume per volume } \\ \text { w/v } & \text { Weight per volume }\end{array}$





\section{Chapter 1: Introduction}

\subsection{Targeted novel anti-cancer therapeutics}

According to the most recent Ministry of Health statistics, cancer is now the leading cause of death in New Zealand (29.4\% of total) (Ministry of Health, 2012), highlighting the need for improved treatment strategies. Conventional treatments for cancer typically involve surgery, radiotherapy, chemotherapy, or some combination thereof; and can be successful particularly when accompanying early cancer detection e.g. the 5 year survival rate of melanoma diagnosed when confined to a primary site is $98.2 \%$ versus $15.1 \%$ when diagnosed post distant metastasis (http://seer.cancer.gov/statfacts/html/melan.html, accessed 22/2/13). However, the still unacceptably high rate of cancer mortality points to a need for improved treatment strategies. A desire for novel therapies has been accompanied by a growing understanding of some of the specific features of the tumour micro-environment, such as local immune suppression (De Visser et al., 2006), and tumour hypoxia (Dachs and Tozer, 2000), which can make a major contribution to treatment failure or relapse. This has raised interest in developing treatments which can more specifically target toxicities to cancer cells, while avoiding the often brutal side effects of standard chemotherapies (Breitbach et al., 2011a; Denny, 2000; Forbes, 2010). One such strategy is to treat patients with viral or bacterial pathogens that can specifically target their toxicity towards cancer cells. The use of these experimental and replication competent therapies brings attendant safety concerns. As such the ability to track the location and behaviour of the therapeutic virus/bacteria inside the patient, and in preclinical settings, would be invaluable. This thesis primarily focuses on developing such imaging capability.

\subsection{Oncolytic viruses as targeted anti-cancer therapeutics}

It was first observed over 100 years ago that, in certain cases, viral infection of a cancer patient could lead to positive therapeutic effect and this was later attributed to the fact that some viruses are able to preferentially infect, replicate within, and directly lyse cancerous cells over healthy tissues (Kelly and Russell, 2007). In the modern era researchers have sought to take advantage of such attributes and various oncolytic viruses from multiple classes have been investigated as anti-cancer treatments, including but not limited to adenovirus (Garber, 2006), herpes viruses (Kaufman and Bines, 2010), reovirus (Kelly et 
al., 2012), poxvirus (Breitbach et al., 2011a) and measles virus (Msaouel et al., 2009a). In some cases treatment involves unmodified viruses that possess a native ability to preferentially infect tumour cells (Gollamudi et al., 2009) although more commonly used are oncolytic viruses that have been specifically adapted or engineered for increased tumour specificity. Such specificity can be achieved by deleting viral genes which are crucial for entry and replication within a healthy cell, but dispensable when infecting disregulated cancer cells. For example, deletion of the viral thymidine kinase forces the virus to rely on high thymidine pools found in many cancers (McCart et al., 2004). In another mechanism, cancer cells that have decreased their sensitivity to interferon mediated apoptotic and growth inhibitory regulatory signalling have simultaneously sensitised themselves to attack from some viruses, as interferon signalling can be a crucial component of a successful cellular anti-viral response (Gollamudi et al., 2009; Stojdl et al., 2000).

Interest in oncolytic viruses is not solely due to their targeted nature but also because of the multi-mechanistic ways in which they effect an anti-tumour response. This can be effected through direct lysis of infected cells, by stimulation of both innate and adaptive anti-tumour immunity (Kaufman et al., 2010) and, for some viruses, by initiation of tumour vasculature collapse (Breitbach et al., 2011b). That these mechanisms are distinct from the toxicity brought about by traditional, often anti-proliferative, chemotherapies suggests that oncolytic viruses may synergise well with current standard treatments (Harrington et al., 2010).

The "gold standard" for such therapies would be efficacy against advanced, treatmentrefractory disease (Breitbach et al., 2010). As this is often metastatic, meeting this standard would require that the virus be able to treat multiple, and often inaccessible or unidentifiable lesions. To date, however, the majority of clinical trials have involved intratumoural delivery (Russell et al., 2012). Some of these have observed that following intratumoural injection distal lesions can also show therapeutic response, thought to be due to viral dissemination in the blood infecting distal legions (Park et al., 2008) or through the development of an anti-tumour immune response (Kaufman et al., 2010). Generally speaking though, to effect sustained anti-tumoural response in metastatic disease is thought to require systemic, intravenous viral delivery. This in turn requires that the virus be able to evade the immune system and disseminate in the blood stream; research to improve such capabilities is ongoing (Russell et al., 2012). Use of viruses 
from classes such as vaccinia, which have evolved for intravenous spread, may also confer more efficacious systemic delivery, for example in a Phase I clinical trial intravenous delivery of the vaccinia virus JX-594 was demonstrated as safe, and the virus showed a dose dependent ability to specifically replicate within tumours (Breitbach et al., 2011a). In addition to all of the technical challenges, progress in intravenous delivery has been slowed by regulatory body safety concerns, which mandate a conservative trial escalation to intravenous administration (Harrington et al., 2010).

Demonstrated clinical utility of oncolytic viral therapies has lagged behind the inherent promise and interest in the modality, with only one clinical approval to date; a oncolytic adenovirus H101 approved for treatment of head and neck cancers in China (Garber, 2006). However numerous viral therapies are in active development or early stage clinical trials with two field leaders in phase III trials (OncoVex ${ }^{\text {GM-CSF }}$ and Reolysin ${ }^{\circledR}$ ), and a third (JX-594) in Phase IIb.

\subsubsection{OncoVex ${ }^{\mathrm{GM}-\mathrm{CSF}}$}

OncoVex ${ }^{\text {GM-CSF }}$ is a herpes simplex virus type $I$ that has been engineered to express granulocyte-macrophage colony-stimulating factor (GM-CSF), to encourage an antitumour immune response by stimulating recruitment of dendritic cells to the tumour (Kaufman et al., 2010). It is currently in Phase III clinical trial for treatment of advanced melanoma and head and neck cancer (Kaufman and Bines, 2010). In a Phase II trial for treatment of stage IIIc and IV melanoma, detectable disease was completely eliminated in eight patients and overall objective clinical responses were seen in $26 \%$ of the 50 patients treated (Senzer et al., 2009). In Jan 2011, based on the strength of Phase II results, BioVex was acquired by the publicly listed pharmaceutical company Amgen for $\$ 425$ million, with the deal containing a potential $\$ 575$ million more in milestone rewards (Schmidt, 2011).

\subsubsection{Reolysin ${ }^{\circledR}$}

Reolysin ${ }^{\circledR}$, developed by Oncolytics Biotech Inc, is a replication-competent form of the reovirus serotype 3 Dearing strain, which preferentially infects, replicates within, and stimulates the apoptosis of cancer cells with activated Ras signalling pathways (Kelly et al., 2012; Strong et al., 1998). Reolysin ${ }^{\circledR}$ in combination with paclitaxel and carboplatin is currently in Phase III clinical trials for the treatment of platinum refractory head and neck cancers. In addition, Reolysin ${ }^{\circledR}$ is in more than 10 ongoing Phase II clinical trials 
covering 12 different cancer indications including non-small cell lung cancer, glioma, sarcoma and melanoma, as either a sole agent or more commonly in combination with chemotherapies or radiotherapies (http://www.oncolyticsbiotech.com/clinical-trials accessed 19/12/2012).

\subsubsection{JX-594}

JX-594 is a Wyeth strain vaccinia virus engineered to express GM-CSF and developed by the private biotherapeutics company Jennerex (Parato et al., 2011). In multiple early stage trials (treating over 160 patients combined) JX-594 has been demonstrated as safe and associated with anti-tumour response, and a recently completed Phase II randomised dose finding trial for treatment of advanced hepatocellular carcinoma reported a difference in median survival of high dose and low dose patients (14.1 versus 6.7 months respectively (Heo et al., 2013)). JX-594 is now in a multi centre PhaseIIB trial which aims to enrol 120 patients, in which intratumoural injection will be used to treat patients suffering from advanced hepatocellular carcinoma who have failed sorafenib treatment, http://clinicaltrials.gov/show/NCT01387555.

\subsection{Bacteria as targeted anti-cancer therapeutics}

Viruses are not the only biological agents known to have innate tumour tropism. In preclinical models bacteria from a growing range of genera have been shown to, following intravenous delivery, preferentially infect and replicate inside of the tumour microenvironment, particularly the central necrotic core (Forbes, 2010). This ability is displayed by a number of facultative anaerobes such as Escherichia coli (Brader et al., 2008) and Salmonella typhimurium (Soghomonyan et al., 2005). Reasons for this are not entirely understood but are thought to involve a combinations of factors including the immune privileged nature of the tumour microenvironment (Yu et al., 2008), that the leaky vasculature and sluggish tumour blood flow aid the ability of blood-borne bacteria to adhere to tumour cells (Forbes et al., 2003) and that the increased available local supply of certain nutrients due to necrotic cell death promotes chemotaxis of the motile bacteria to such regions (Kasinskas and Forbes, 2007). This nutrient pool can be targeted to increase tumour specific localisation in these facultative anaerobes by gene-knockout to generate auxotroph strains (Zhao et al., 2005), for example the clinically tested Salmonella typhimurium VNP20009 strain carries a purI deletion that forces the bacteria to rely on external sources of adenine (Toso et al., 2002). 
For obligate anaerobes such as Clostridium (Liu et al., 2008b) or Bifidobacterium (Li et al., 2003) the primary mechanism for tumour targeting is better understood, as these bacteria can only replicate at pathologically low oxygen levels i.e. in the hypoxic and necrotic core of solid tumours. Therapeutically this is beneficial, as hypoxia is a well established negative prognostic factor for tumour treatment, but does not typically occur in healthy tissue (with the exception of the bone marrow compartment (Asosingh et al., 2005)) providing a chance to target toxic therapies specifically to cancerous cells. Hypoxia is a state of abnormally low oxygen levels (typically defined as an oxygen partial pressure of 2.5-10 mm Hg (Rasey et al., 1999), which arises to at least some extent in the majority of solid tumours larger than $1 \mathrm{~mm}^{3}$ (Dachs and Tozer, 2000) due to rapid tumour growth and irregular vasculature compromising blood supply (Vaupel et al., 1989). Hypoxic cells are resistant to radiotherapy, which requires generation of oxygen free radicals for full effect (Gray et al., 1953). Hypoxia also increases resistance to chemotherapeutic treatment as the slow growing nature of many hypoxic cells spares these cells from anti-proliferative chemotherapies (Kennedy et al., 1997) and as the compromised vasculature associated with hypoxia limits drug delivery (Denny, 2000). Hypoxia is also known to alter patterns of cell gene expression to promote a more aggressive cancer phenotype (Dachs and Tozer, 2000). As motile bacteria actually penetrate deep into the hypoxic/necrotic tumour core (Kasinskas and Forbes, 2007; Umer et al., 2012), this provides a method of delivering therapies specifically to problematic areas that should synergise well with existing chemotherapies whose diffusion to such areas is limited (Forbes, 2010).

In preclinical models bacteria have been shown to exert direct anti-tumour effects (Bettegowda et al., 2003; Weibel et al., 2008; Zhao et al., 2007), however it is primarily envisaged that for clinical use these effects would be augmented by engineering the bacteria to express proteins that confer enhanced toxicity (Forbes, 2010; Hoffman, 2011). One strategy is to engineer the bacteria to express directly toxic proteins such as TNFrelated apoptosis-inducing ligand (Ganai et al., 2009) or to increase immune stimulation through expression of cytokines such as interleukin-2 (al-Ramadi et al., 2009). An additional strategy is to "arm" the bacteria to express an enzyme that is capable of converting a systemically delivered benign prodrug into a potent chemotherapeutic (King et al., 2009; Theys et al., 2006). Broadly speaking this strategy is termed gene directed enzyme prodrug therapy (GDEPT). 


\subsection{Gene directed enzyme prodrug therapy}

GDEPT refers to experimental anti-cancer gene therapies in which a tumour targeting vector, most commonly viral, is used to deliver a gene specifically to tumour cells, wherein the enzyme product of this gene is then expressed. This enzyme is able to convert a non-toxic prodrug into a potent chemotherapeutic - thus targeting the toxicity of the compound specifically towards cancer cells. Crucially, the activated chemotherapeutic should be able to diffuse to and kill closely neighbouring cancer cells, conferring the possibility of achieving significant tumour ablation without having to achieve the (essentially impossible) aim of transfecting every tumour cell with the delivered gene Figure 1-1. This "bystander effect" can provide an attractive adjunct to oncolytic virotherapies as it confers an additional mechanism to destroy tumour cells that have not been directly infected with the virus (Kirn and Thorne, 2009).

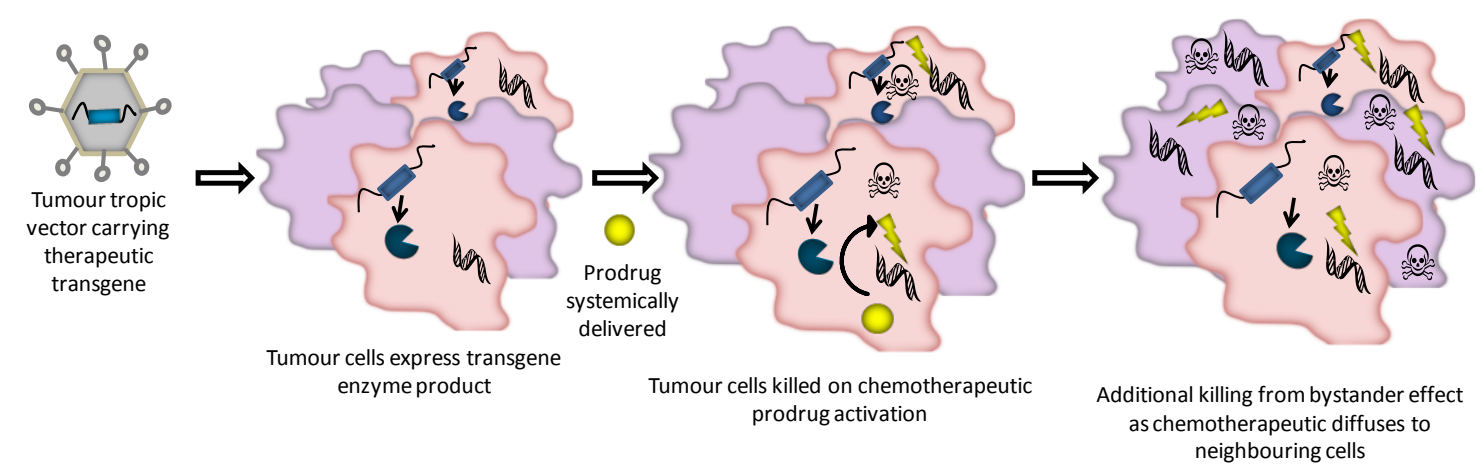

Figure 1-1 Schematic showing principle of gene directed enzyme prodrug therapy. A tumour tropic vector (in this example a virus), is used to deliver the therapeutic transgene specifically to tumour cells, which if transfected will then express the enzyme. When the systemically delivered prodrug encounters this enzyme, it is converted to a potent chemotherapeutic which is also able to diffuse to neighbouring tumour cells.

A number of possible enzyme/prodrug combinations have been investigated (Altaner, 2008). The most trialled enzyme/prodrug modality, the use of herpes simplex virus thymidine kinase (HSV1-TK) to activate the nucleoside analogue ganciclovir (GCV) is described in detail below (section 1.4.1). Other notable modalities include cytosine deaminase mediated conversion of 5-fluoroctyosine to the nucleoside analogue 5fluorouracil (Freytag et al., 2007), the activation of DNA damaging mustard prodrugs by cytochrome P450 variants (Ma and Waxman, 2007) and the modality most relevant to this 
thesis, the use of bacterial nitroreductase (NTR) enzymes to activate nitroaromatic DNAdamaging prodrugs.

\subsubsection{Herpes simplex virus thymidine kinase and ganciclovir}

HSV1-TK/GCV was the first suicide enzyme/prodrug pair suggested (Moolten, 1986) and has been the most widely investigated (Duarte et al., 2012). Triphosphorylated GCV can cause cell death by interfering with DNA replication via direct inhibition of DNA polymerase and by misincorporation into elongating DNA strands. Retroviral delivery of HSV1-tk followed by GCV therapy was incorporated in the treatment of glioblastoma multiforme in the first GDEPT phase III clinical trial (Rainov, 2000). While in this instance no additional therapeutic benefit was observed, clinical research has continued using different gene delivery strategies, and a number of Phase I and II clinical trials incorporating this treatment have seen responses suggestive of improvement in patient outcome (Freytag et al., 2007; Li et al., 2007; Nasu et al., 2007). In a more recent example of application of this therapeutic strategy, a replication competent adenovirus expressing both an engineered mutant version of $H S V 1-t k$, a yeast derived cytosine deaminase gene, and a viral protein that enhances oncolytic activity is currently in a randomised Phase II/III trial for treatment of prostate cancer in combination with valganciclovir (a pre-prodrug of gancicovir), 5-fluorocytosine and radiotherapy (Lu et al., 2011). It should be noted that one disadvantage of the HSV1-TK system is that as the chemotherapeutic is a nucleoside analogue only actively dividing cells are targeted (the same limitation applies to cytosine deaminase). An added disadvantage is that activated GCV is not freely cell permeable, making the bystander effect of the treatment primarily dependant on cell-cell contacts such as gap junctions (Mesnil and Yamasaki, 2000), which can be down-regulated in the dysfunctional tumour environment (Trosko, 2003).

\subsubsection{Bacterial nitroreductase NfsB and CB1954}

The prototypical NTR/prodrug pair is the E. coli enzyme NfsB and the aziridinyl dinitrobenzamide prodrug CB1954. Interest in CB1954 was triggered when it was observed as a sole agent to efficiently treat tumours arising from a transplanted Walker256 rat carcinoma cell line (Cobb et al., 1969). This potent tumour toxicity was later found to stem from CB1954 acting as a tumour activated prodrug, with reduction of CB1954 by tumour upregulated rat NQO1 converting the weak monofunctional alkylating agent to a 4-nitrohydroxylamine $(\mathrm{NHOH})$ derivative. This derivative, following further interaction with intracellular thioesters such as acetyl CoA, (either via non-specific (Knox 
et al., 1991) or enzymatic means (Mitchell and Minchin, 2008)) becomes a potent DNAcross linker (Figure 1-2) .<smiles>CC(=O)ONc1cc([N+](=O)[O-])c(C(N)=O)cc1N1CC1</smiles>

Figure 1-2 CB1954 reduction by rat NQO1 at the 4-nitro moiety converts the compound to first the nitroso (not pictured) and then nitrohydroxylamine derivative. Non-specific reaction of the latter product with intracellular thioesters results in a compound that binds to and cross-links DNA (Knox et al., 1991).

Unfortunately CB1954 is not suitable for use as a sole chemotherapeutic agent against human tumours, which do not exhibit the same CB1954 sensitivity, due to the inability of the human form of NQO1 to efficiently reduce the prodrug (Boland et al., 1991). However another possible application of CB1954 therapy became apparent when the $E$. coli NTR enzyme NfsB was shown to reduce CB1954 90-fold faster than even rat NQO1 (Anlezark et al., 1992), suggesting utility as a prodrug for GDEPT. Importantly, the reduced cytotoxic metabolites of CB1954 are cell permeable and demonstrate a bystander effect (Bridgewater et al., 1997), thought to be primarily effected by the reduced metabolites that arise from reduction of the 2-nitro group (Helsby et al., 2004). NfsB can reduce either nitro group with equal efficiency, generating equimolar mixes of the 2hydroxylamine and 4-hydroxylamine reduction products (Anlezark et al., 1992).

Phase I/II trials of CB1954 in conjunction with a replication defective-adenovirus expressing E. coli NfsB showed that treatment was safe, and a decline in levels of prostate specific antigen in some patients suggested some effect, however overall it was deemed that greater therapeutic efficacy would be desirable (Patel et al., 2009). While next generation viral vectors would presumably be beneficial to this therapy, trial data also suggested that the combination of CB1954 and NfsB may not be optimal. Hepatotoxicity in clinical trials limited the maximum tolerated drug dose, and achievable peak serum concentrations of CB1954 $(6.3 \pm 2.8 \mu \mathrm{M})$ (Chung-Faye et al., 2001) were less than one eightieth of the lowest reported $K_{m}$ for E. coli NfsB (Anlezark et al., 1992). As such, a search for both more efficient NTRs and effective prodrugs is warranted. 


\subsection{Strategies to improve NTR based GDEPT}

\subsubsection{Alternative nitroaromatic prodrugs}

NTR based GDEPT using the prodrug CB1954 does have the advantage of building off decades of research, as well as the pharmacokinetic data and compliance with good manufacturing practice conferred by phase I/II clinical trials (Chung-Faye et al., 2001; Patel et al., 2009). However another drug class also offers the same advantages, the hypoxia activated dinitrobenzamide mustards. Of these the lead candidate PR-104 (Figure 1-3), successfully passed Phase I clinical trial (Jameson et al., 2010; McKeage et al., 2011) as a hypoxia-activated therapeutic (i.e. independent of GDEPT application).<smiles>COCCNC(=O)c1cc([N+](=O)[O-])cc([N+](=O)[O-])c1N(CCBr)CCOC</smiles>

Figure 1-3 Chemical structure of the dinitrobenzamide mustard PR-104

Activated nitrogen mustards are extremely potent drugs due to their ability to bind to the $\mathrm{N}^{7}$ position of guanine and subsequently cross-link DNA (Mattes et al., 1986) (Figure 1-4), and were in-fact among the first small molecules ever used to treat cancer (DeVita and Chu, 2008).

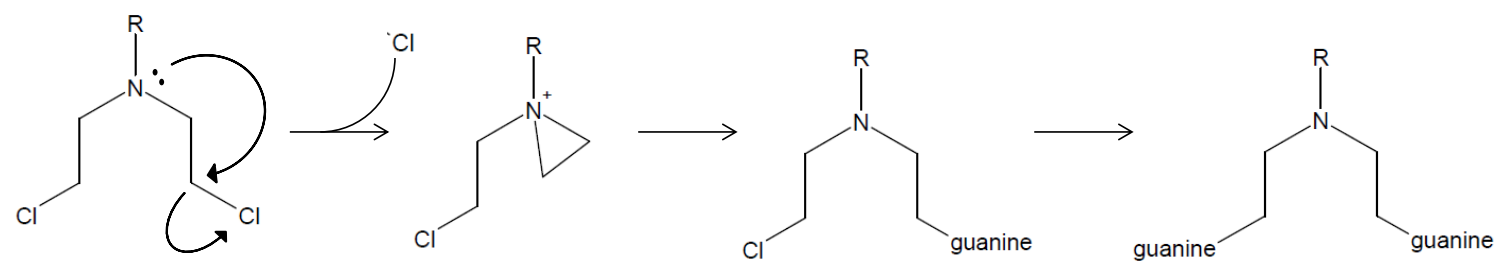

Figure 1-4 DNA cross-linking by a nitrogen mustard, shown here with chlorine leaving groups (Wardman, 2001).

The molecular basis for the use of dinitrobenzamide mustards as hypoxia activated prodrugs is that this potent toxicity can be "harnessed" by attachment of the mustard 
moiety to an electron withdrawing dinitrobenzamide ring, thus stabilising the molecule. However if a nitro group on the dinitrobenzamide ring is reduced, this change in electron density converts the dinitrobenzamide ring from an electron withdrawing to an electron donating group, re-activating the inherent toxicity of the mustard (Denny and Wilson, 1993; Guise et al., 2007a).

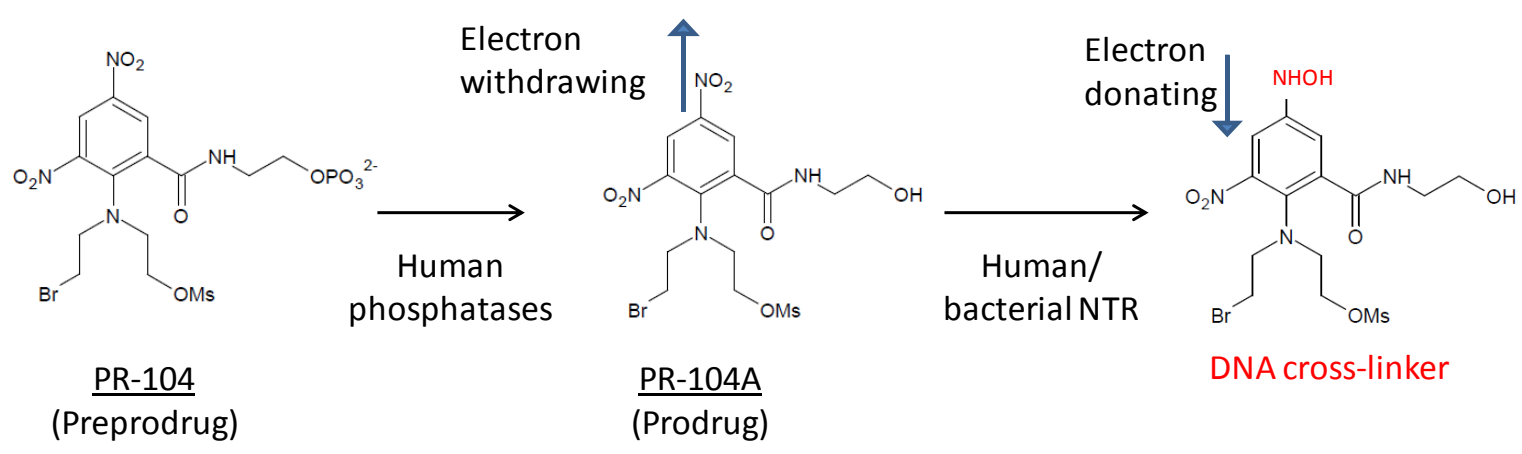

Figure 1-5 Reduction of PR-104. PR-104 is administered in "pre-prodrug" form, with an attached phosphate that increases solubility in the bloodstream. This is cleaved in vivo by human phosphatases to generate the more lipophilic prodrug PR-104A. Reduction of the para nitro group to either hydroxylamine or amine form (as per Figure 1-6) converts the dinitrobenzamide ring from an electron withdrawing to an electron donating group, activating the latent toxicity of the nitrogen mustard substituent.

The basis of hypoxia activation in vivo is that the dinitrobenzamide mustards are substrates for a number of endogenous human enzymes (e.g. cytochrome P450 reductase (Guise et al., 2007a)) that can carry out reduction in one electron increments. This mechanism of reduction produces an unstable one electron radical as the first reduction product, which in the presence of oxygen is rapidly back-oxidised to the original prodrug. This means that full reduction to a DNA cross-linking metabolite can occur only in the absence of oxygen. In contrast, NfsB and other oxygen insensitive NTRs reduce nitroaromatic substrates in simultaneous 2e- increments (Bryant et al., 1981; Koder et al., 2002; Peterson et al., 1979), meaning production of reduced metabolites in a GDEPT context is independent of the presence of oxygen (Figure 1-6). 


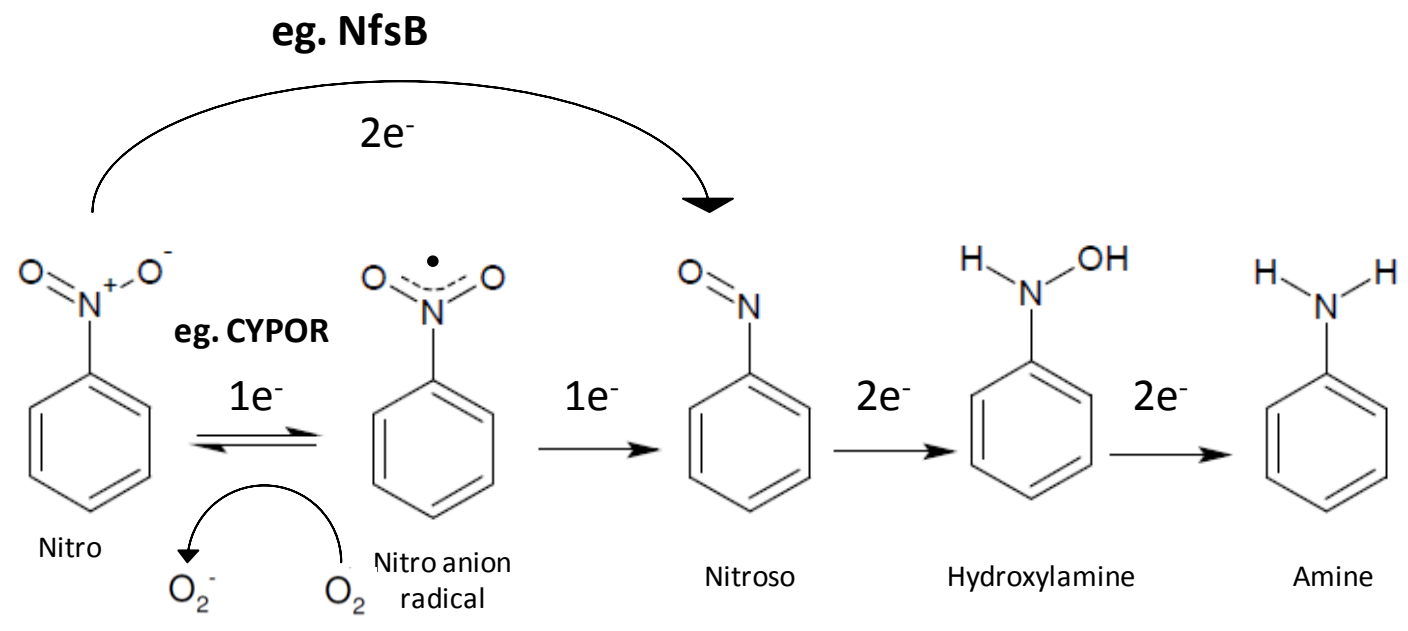

Figure 1-6 Schematic of hypoxia dependent and independent nitroaromatic reduction, proceeding from the parental compound to an amine derivative in $1 \mathrm{e}^{-}$and/or $2 \mathrm{e}^{-}$steps. If the first reduction is carried out by a 1e $\mathrm{e}^{-}$reductase (e.g. cytochrome P450 reductase (CYPOR)) the full reduction can only proceed in the absence of oxygen, otherwise the resulting nitro anion radical is back oxidised, regenerating the parental compound in a futile cycle. In contrast if all reduction is carried out in $2 \mathrm{e}^{-}$increments, as catalysed by bacterial NTRs (e.g. NfsB), then reduction proceeds irrespective of oxygenation status.

Disappointingly, the safe clinical dose of PR-104 was lower than had been anticipated, which may limit its efficacy as an independent chemotherapeutic agent (McKeage et al., 2011). PR-104 was found to undergo off-mechanism aerobic (2e-) reduction by human aldo-keto reductase 1C3, going some way to explain this observation (Guise et al., 2010) and suggesting that dinitrobenzamide mustards that are not substrates for human aldoketo reductase 1C3 may be more suited for sole agent use. Despite the off-mechanism AKR1C3 activity PR-104 is still far more dose potent than CB1954, with an up to 20 times higher maximum tolerated dose on a molar basis (Jameson et al., 2010). This, coupled with the advanced clinical status of PR-104, makes it an attractive (pre)prodrug for GDEPT.

Various NTRs, including NfsB, show better catalytic activity with PR-104A (Prosser et al., 2013) and other dinitrobenzamide mustards (Anlezark et al., 1995) than they do with CB1954, an observation that also suggests these molecules may prove more appropriate prodrugs for GDEPT. Importantly, dinitrobenzamide mustards show substantially greater potency and bystander effect in mixed population 3D cell models (which measure the level of bystander cell death caused by activated drug diffusing from a small population 
of NTR expressing cells), largely due to their increased lipophilicity compared to CB1954 (Atwell et al., 2007; Singleton et al., 2007; Wilson et al., 2002). This increased potency is reflected in vivo, for example mice carrying tumour xenografts containing $10 \% \mathrm{NfsB}$ positive cells showed an increase in median survival of only 11 days following CB1954 treatment compared to 62 days with a dinitrobenzamide mustard SN28343 (Singleton et al., 2007).

\subsubsection{Alternative bacterial nitroreductases for GDEPT}

A different but complementary approach to improving NTR GDEPT is to identify NTRs that better activate CB1954 and/or other nitroaromatic prodrugs such as PR-104. This has been pursued by researchers investigating the prodrug activating abilities of bacterial nitroreductases found in nature, both by examining NfsB homologues (Emptage et al., 2009; Swe et al., 2012; Theys et al., 2006) and NTRs that share little sequence similarity to NfsB such as Bacillus amyloliquefaciens YwrO (Anlezark et al., 2002) and homologues (Theys et al., 2006) or an engineered derivative of E. coli YieF (Barak et al., 2006). NfsB has been shown to be less effective at sensitising tumour cells to CB1954 than another E. coli enzyme, termed NfsA, (Vass et al., 2009), and at a purified protein level NfsA shows a near 10-fold higher specificity constant for CB1954 reduction (Prosser et al., 2010). More effective GDEPT NTRs have also been sought by generating mutants of NfsB with improved activities towards CB1954 (Guise et al., 2007b; Jaberipour et al., 2010). Some of these engineered NfsB derivatives are discussed in more detail in Chapter 6 of this thesis.

\subsection{Bacterial nitroreductases}

Bacterial NTRs are a ubiquitous and diverse enzyme class, with the best characterised NTRs belonging to the NfsA (Liu et al., 1997; Nokhbeh et al., 2002; Zenno et al., 1996a) or NfsB subfamilies (Anlezark et al., 1992; Bryant and DeLuca, 1991; Koike et al., 1998). While there is essentially no sequence identity shared between these two families, both types of enzyme function as FMN dependent homodimers. NTRs exhibit bi-bi ping pong kinetics, meaning overall substrate reduction proceeds via NADPH binding, transfer of 2e- from $\mathrm{NAD}(\mathrm{P}) \mathrm{H}$ to reduce the associated FMN cofactor and exit of the $\mathrm{NAD}(\mathrm{P})+$ from the active site; followed by binding of the second substrate close to the $\mathrm{FMNH}_{2}$ and subsequent transfer of the 2e- to reduce the second substrate (Koder and Miller, 1998; Zenno et al., 1996a). NfsA family members typically exhibit a pronounced preference for 
NADPH (Liu et al., 1997; Morokutti et al., 2005; Nokhbeh et al., 2002), whereas the NfsB family can use either co-factor (Koder and Miller, 1998; Zenno et al., 1994). That the reduction is carried out in obligate 2e- steps is believed to be because the $1 \mathrm{e}$ - reduced form of FMN is highly disfavoured while this cofactor is NTR associated (Koder et al., 2002).

NTRs are extremely promiscuous enzymes, and due to their broad substrate specificity are also under investigation for bioremediation, as they can reduce (and thereby detoxify) the pollutant chromate (Ackerley et al., 2004) or nitroaromatic explosives such as TNT (Williams et al., 2004). Certain NTR subfamilies can also reduce and cleave azo bonds, making them potentially useful in degrading recalcitrant azo-containing commercial dyes (Liu et al., 2007).

It is self evident that while the reduction of anthropogenic substances may be of biotechnological interest, it is not for such purposes that this widespread enzyme class evolved. As this thesis focuses on the ability of these enzymes to reduce nitroaromatic substrates they have been designated throughout with the term nitroreductase; but when tested directly they are typically more catalytically active with quinone substrates (Zenno et al., 1996a, 1996b). Quinones are prevalent in nature, and oxygen insensitive NTRs reduce quinones to their hydroquinone form in two electron steps. This bypasses the extremely reactive semi-quinone intermediate and thereby minimises ROS generation. It has been suggested that such a protective mechanism may in fact be the primary biological role of many of these enzymes (Deller et al., 2008; Liu et al., 2008a; Roldán et al., 2008). Consistent with this hypothesis, it has been shown that NfsA from E. coli is part of the soxRS (superoxide response) operon, an operon which includes at least 15 genes implicated in responding to oxidative stress (Liochev et al., 1999). Moreover, nitroreductases in various bacterial strains are induced or prove protective in the face of such stressors as $\mathrm{H}_{2} \mathrm{O}_{2}$, paraquat and heat shock (Gonzalez et al., 2005; Liochev et al., 1999; Liu et al., 2008a; Moch et al., 2000; Nokhbeh et al., 2002). In some bioluminescent bacteria however, e.g. Vibrio fischeri and Vibrio harveyi, NTRs act to maintain a pool of reduced flavin monomers, required for luciferase activity (Zenno et al., 1994; Tanner et al., 1996).

In addition to these known activities, a previously uncharacterised activity of these bacterial NTRs may offer the possibility of using these enzymes to noninvasively image 
the location and behaviour of a therapeutic virus/bacteria inside patients, and in preclinical settings.

\subsection{Non-invasive imaging of anti-cancer gene therapy}

It is widely recognised that the ability to non-invasively image the location and spread of these novel, complex and in some cases replication competent, therapies would be extremely beneficial for their clinical development (Baril et al., 2010; Barton et al., 2008; Bhaumik et al., 2011; Chiocca, 2002; Rojas and Thorne, 2012). Such imaging would have to encompass the whole body and be sensitive and repeatable to allow for visualization of vector location over time.

Current clinical practices for monitoring location and levels of therapeutic vector typically consist of biopsy analysis of treated patients (Adair et al., 2012; Breitbach et al., 2011a; Patel et al., 2009) and/or PCR based determination of levels of viral DNA/RNA in a patients plasma, urine and stool (Gollamudi et al., 2009; Immonen et al., 2004; Nasu et al., 2007). This is far from ideal; plasma virus levels give no indication of vector location, and in addition to patient discomfort the invasive nature of biopsy precludes the possibility of sampling over time and restricts locations where gene delivery can be assessed, meaning full determination of vector behaviour over time and detection of any off target delivery is near impossible. These considerations are particularly important for demonstrating the safety and efficacy of systemically delivered replication competent vectors, which offer the best chance to treat advanced metastatic disease (Breitbach et al., 2011a; Garber, 2006). Non-invasive whole body imaging would also allow a more accurate determination of the efficacy of a treatment, as clinicians would be able to assess patient response in light of the level of vector delivery achieved. In GDEPT based protocols, such imaging would help determine the optimal time point for prodrug administration. In addition to clinical utility, vector imaging would of course be extremely useful for assessing efficacy in experimental and preclinical settings.

Non-invasive whole body imaging has been largely pursued by engineering the therapeutic vector to carry a reporter gene. The protein product of this gene is then detected, typically by application of an exogenous substrate, a reporter probe, which can subsequently be visualized by the appropriate imaging technology. A number of strategies for imaging anti-cancer gene therapies are discussed below. 


\subsubsection{Optical imaging}

Optical imaging involves the emission and subsequent detection of a bioluminescent or fluorescent signal. Due to its low cost and relative ease of application, optical imaging has been widely used to successfully monitor transgene expression and vector localisation in both in vitro and small animal studies. When fluorescence is used in reporter gene imaging, the gene product can either fluoresce independently, e.g. GFP (Chen et al., 2010), or can generate a fluorescent signal by converting an exogenously delivered probe to a fluorescent form (Bhaumik et al., 2011). An in vivo signal can be best monitored when emitted fluorescence is in the red or better yet near-infrared (650-900 nm) spectrum, as these light frequencies are better able to penetrate tissue and avoid much of the background signal generated by tissue auto-fluorescence at shorter wavelengths (Nolting et al., 2011) .

Bioluminescence, wherein surface light emission due to a reporter gene is captured using cooled charged coupled device (CCD) cameras, has been used by a number of laboratories to monitor in vivo vector localization in small animal cancer models. Bioluminescent imaging can be extremely sensitive, as there is next to no background signal, but it suffers from low spatial resolution and is generally used to generate 2D images, although 3D reconstruction is possible (Cronin et al., 2012; Kuo et al., 2007). Most commonly bioluminescent signal is generated by reporter "luciferase" proteins such as firefly or Renilla luciferase which in conjunction with oxygen interact with exogenously delivered "luciferin" substrates to generate light emission as a by-product (Bhaumik and Gambhir, 2002). Engineering of a number of bacterial species to carry the lux $C D A B E$ gene cluster (which allows for continuous self-sufficient light emission) has also been used for monitoring bacterial tumour colonisation in small animal models (Cronin et al., 2012; Yu et al., 2004).

Ultimately though, however useful these techniques are in pre-clinical work, they are intrinsically unsuited to whole body imaging in a clinical setting as optical signal attenuation due to tissue absorption gives a depth limit of centimetres at best (Chen et al., 2010; Razgulin et al., 2011).

\subsubsection{Magnetic resonance imaging}

Magnetic resonance imaging (MRI) is clinically used to provide non-invasive anatomical images, constructed by detecting tissue dependent differences in behaviour of nuclei 
(typically the hydrogen nucleus) when exposed to the strong magnetic field generated by a MRI scanner. MRI can be used to detect reporter gene expression if the protein product can affect the behaviour of immediately surrounding nuclei, for example by manipulating local concentrations of paramagnetic iron (Hill et al., 2011; Zurkiya et al., 2008). While MRI has higher spatial resolution, it is less sensitive i.e. less able to detect low levels of reporter probe, than nuclear imaging (discussed below; section 1.7.4), and it has not yet received the same attention, development and validation for imaging of tumour tropic vectors (Gilad et al., 2008; Hill et al., 2011).

\subsubsection{Ultrasound imaging}

Ultrasound imaging can be adapted for non-invasive imaging of gene delivery, by codelivery of the gene with ultrasound detected microbubbles (Sirsi, 2012), but this technique as it stands is not appropriate for the imaging of the in vivo behaviour of biological vectors.

\subsubsection{Nuclear imaging}

In nuclear imaging a small amount of radioisotope labelled tracer is administered systemically to the patient. The tracer is designed so that interaction with a reporter protein causes it to accumulate in the cell, whereas tracer that has not encountered the reporter protein is cleared from the body. The location and level of the remaining cellsequestered tracer can then be visualized using specialized scanners that detect its radioactive decay (Figure 1-7). 


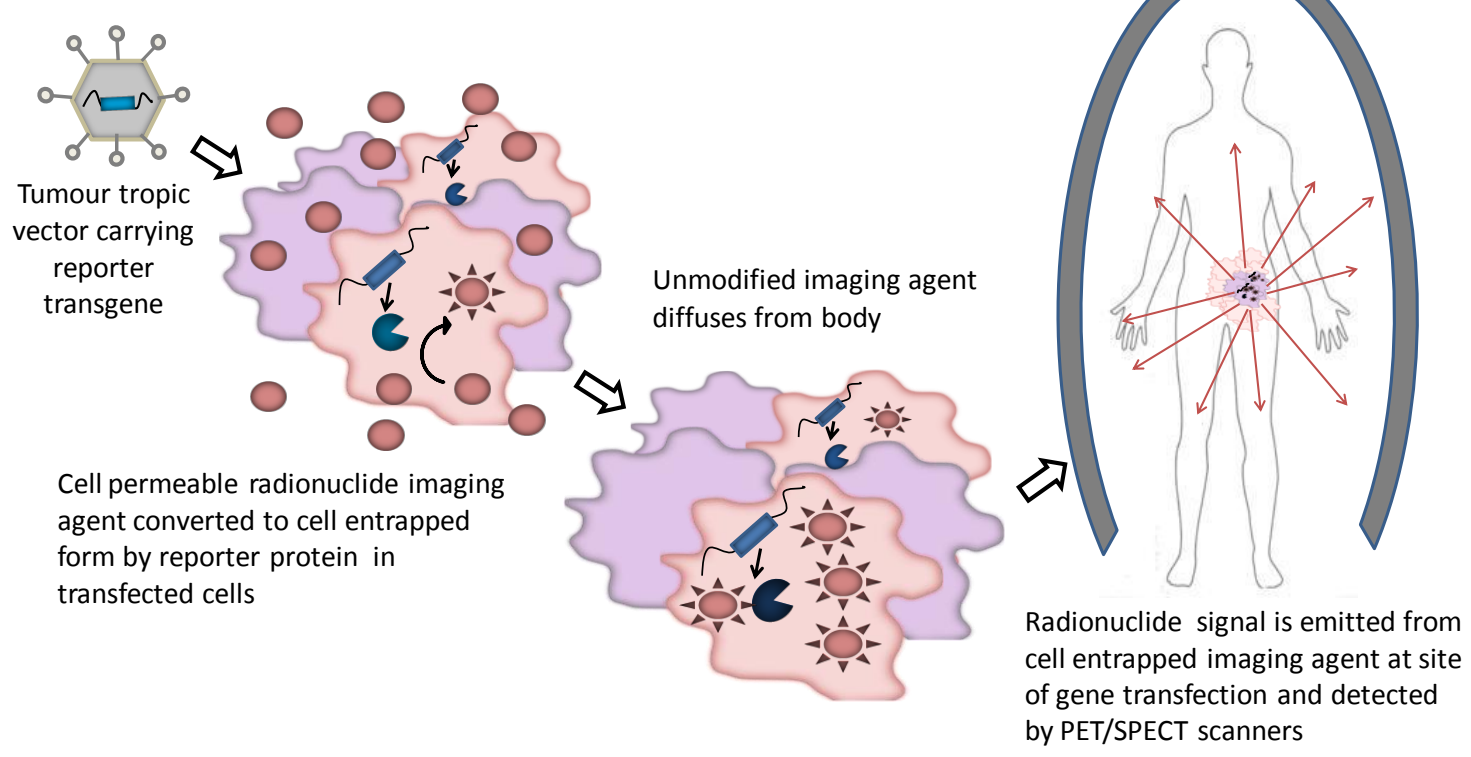

Figure 1-7 Schematic showing the nuclear imaging of a reporter gene/probe. In this example the reporter protein enzymatically modifies the radioisotope labelled probe to a cell entrapped form, the signal of which can be detected by PET/SPECT scanners enabling non-invasive identification of reporter gene location and thus the location of the tumour tropic vectors.

The radioisotope chosen to label the tracer determines whether positron emission tomography (PET) or single photon emission computed tomography (SPECT) is used. In PET scanning the radioisotope emits positrons, which quickly collide with electrons as they travel through the surrounding tissue. This collision causes two high energy gamma rays $(511 \mathrm{keV})$ to be simultaneously released at a precise (near $\left.180^{\circ}\right)$ angle from each other. This 'coincidence' event is registered by surrounding detectors and used to reconstruct a precise tomographical image of the location and level of the reporter probe (Briat and Vassaux, 2006; Rahmim and Zaidi, 2008). Radioisotopes detectable by PET include ${ }^{18} \mathrm{~F},{ }^{11} \mathrm{C},{ }^{124} \mathrm{I},{ }^{64} \mathrm{Cu},{ }^{13} \mathrm{~N}$ and ${ }^{15} \mathrm{O}$ (Waerzeggers et al., 2009). In the late 1990s, integrated PET/CT scanners were developed (Beyer et al., 2000) and the vast majority of PET scans are now conducted in concert with commuted tomography (CT) scans to give anatomical images that are precisely combined with the PET derived functional information. SPECT detects radioisotopes such as ${ }^{123} \mathrm{I},{ }^{99 \mathrm{~m}} \mathrm{Tc}$ or ${ }^{111} \mathrm{In}$, which directly emit low energy gamma rays. These are detected by a gamma camera which is rotated around the patient to provide whole body images of the source of this decay. To allow localization of the origin of a signal, SPECT cameras are fitted with lead collimators, which only allow rays approaching at a precise angle to hit the camera. The consequent 
blocking of a large proportion of signal means that SPECT is inherently less sensitive than PET (Briat and Vassaux, 2006; Min and Gambhir, 2008).

Nuclear imaging has a number of distinct advantages that have led many commentators to suggest it as the most appropriate technology for clinical non-invasive whole body imaging (Briat and Vassaux, 2006; Peñuelas et al., 2005a; Ponomarev, 2009). It is highly sensitive, quantitative, repeatable, can give reasonable spatial resolution (3-4 mm), and has no clinically relevant depth limit (Razgulin et al., 2011; Waerzeggers et al., 2009). The primary drawback to nuclear imaging is the often complicated generation and handling of radionuclide probes, and the need for specialized dedicated instrumentation and personnel. One advantage of SPECT over PET is that radioisotopes suitable for SPECT are typically more stable (hour-to-day radioactive half-lives vs minute-to-hour half-lives for the more commonly used PET radioisotopes) (Hicks and Hofman, 2012). Moreover, synthesis of some of the most common SPECT radioisotopes, such as ${ }^{99 \mathrm{~m}} \mathrm{Tc}$, does not require access to a medical cyclotron (Dokić, 2005). However, as previously mentioned SPECT is inherently less sensitive than PET, detecting only around $0.01 \%$ of total photons as opposed to $1 \%$ by PET (Rahmim and Zaidi, 2008). PET also offers greater spatial resolution and a more quantitative report on probe levels (Hicks and Hofman, 2012; Min and Gambhir, 2008).

While PET-suitable radioisotope synthesis is more challenging, requiring the use of medical cyclotrons to bombard stable atoms to create short-lived radioisotopes, clinical use of PET is increasing. The number of clinical PET scans conducted p.a. in the USA increased over the period 2004-2010 from $\sim 900,000$ to $>1.7$ million and there are $\sim 2200$ clinical PET centres worldwide (Yuin et al., 2012). Increased use generally drives down costs, and it is reported that for a high throughput facility the purchasing and operating of a PET scanner has seen a $>50 \%$ decrease in real cost over the last 10 years (Hicks and Hofman, 2012). This growth has been largely driven by the clinical adoption of PET/CT imaging of $\left[{ }^{18} \mathrm{~F}\right] \mathrm{FDG}$ bio-distribution for identification of metastases and staging of certain cancers (Herrmann et al., 2011). Over 90\% of PET scans are conducted to monitor $\left[{ }^{18}\right.$ F]FDG (Coenen et al., 2010). However the investment in the infrastructure and technology required for $\left[{ }^{18} \mathrm{~F}\right] \mathrm{FDG}$ production and imaging can be leveraged to support more niche applications of ${ }^{18} \mathrm{~F}$ based PET/CT imaging, such as the monitoring of gene therapy vectors. 
A variety of existing reporter protein/probes are already under investigation and promote cell specific localization of radionuclide probes through one of three different mechanisms (Briat and Vassaux, 2006). Proteins such as HSV1-TK can enzymatically modify a cell permeable probe to a cell entrapped form (Yaghoubi and Gambhir, 2007); transmembrane proteins such as human sodium iodide symporter (hNIS) can actively cause cells to uptake unmodified probes (Barton et al., 2011); and finally radionuclide probes can accumulate by binding to specific cell receptors, such as the human type II somatostatin receptor (Kim et al., 2012). A number of investigated reporter genes such as the human type II somatostatin receptor and the human norepinephrine transporter (Brader et al., 2009a) carry the advantage that their nuclear imaging probe counterparts have already been clinically used for other imaging applications. However, to date the two most prominent reporter genes to have been tested for nuclear imaging of vectors in oncology applications are HSVl-tk and $h N I S$.

\subsubsection{HSV1-TK}

HSV1-TK can phosphorylate and thereby entrap within the host cell a number of radiolabelled nucleoside analogues, allowing for nuclear imaging of the location of active HSV1-TK. To date HSV1-tk (both wild type and mutant variants) has been the most widely used reporter gene for nuclear imaging. Radiolabelled substrates include the SPECT imageable ${ }^{125}$ I-FIAU and PET imageable pyrimidine derivatives such as $\left[{ }^{18} \mathrm{~F}\right]$ FEAU, $\left[{ }^{24} \mathrm{I}\right]$ FIAU and the acycloguanosine derivative $\left[{ }^{18} \mathrm{~F}\right]$ FHBG, which has FDA approval as a investigational new imaging agent (Yaghoubi and Gambhir, 2007).

Non-invasive imaging of $H S V 1$-tk expression was first accomplished by using SPECT to monitor $\left[{ }^{131}\right.$ I]FIAU accumulation in tumours expressing HSV1-tk in a rat cancer model (Tjuvajev et al., 1996). Nuclear imaging of the expression of HSVI-tk or a mutant derivative HSVI-sr39tk (Gambhir et al., 2000) has subsequently been used both to support preclinical development of HSV1-TK anti-cancer therapies (Abate-Daga et al., 2011; Fontanellas et al., 2009) and to investigate the possible detection of tumour metastases by imaging of HSVl-tk carrying tumour tropic vectors (Brader et al., 2009b). The first report of clinical non-invasive imaging of HSVI-tk was in 2001, where as part of a HSV1-TK/GCV trial to treat recurrent glioblastoma, five patients were also delivered $\left[{ }^{124}\right.$ I]FIAU and tumour specific accumulation was detected in one patient using PET (Jacobs et al., 2001). Subsequently in a Phase I trial involving treatment of hepatocellular carcinoma by intratumoural injection of a replication deficient adenovirus carrying HSVI- 
$t k$, followed by GCV application, it was reported that patients who received a dose of over $10^{12}$ virus particles showed a clear PET imageable accumulation of intravenously administered $\left[{ }^{18}\right.$ F]FHBG only in the tumour treated with virus (Peñuelas et al., 2005b). HSV1-TK based PET imaging has also been applied to the monitoring of infused therapeutic cytolytic T-cells (Yaghoubi et al., 2009) and HSV1-tk delivery through cationic liposome vectors (Sangro et al., 2010). It should be noted that there has also been one report in which the attempted non-invasive imaging of the replication of a herpes simplex virus aimed at treating glioma was unsuccessful, though this has been suggested to be a consequence of the less sensitive SPECT modality used to detect ${ }^{123}$ I-FIAU accumulation (Dempsey et al., 2006).

\subsubsection{Preclinical nuclear imaging of bacterial cancer therapies}

Thymidine kinase activated radionuclide probes have been used to non-invasively image tumour targeting bacteria in preclinical studies. The clinically trialled Salmonella VNP20009 strain, modified to carry HSVl-tk, can be localised using ${ }^{124}$ I-FIAU and microPET (Soghomonyan et al., 2005). Non-invasive imaging of Salmonella would be particularly beneficial, as in clinical trial it appeared that the fine needle biopsies taken to measure in vivo bacterial colonisation did in some instances not detect bacteria later shown to be present, possibly because of the bacteria's irregular hypoxic/necrotic dependent intratumoural localisation (Toso et al., 2002). Interestingly it has also been shown that some bacterial species do not require addition of HSVI-tk for imaging, as their endogenous thymidine kinases exhibit sufficient phosphorylation of radionucleotide probes. This property was used to image the localisation of the non-pathogenic E. coli Nissle 1917 strain in tumour bearing mice (Brader et al., 2008) and the tumour specific germination in BALB/c mice of the clinically relevant $C$. novyi-NT following $\left[{ }^{125}\right.$ I]FIAU treatment and SPECT/CT imaging (Bettegowda et al., 2005).

\subsubsection{Human sodium iodide symporter}

The human sodium iodide symporter (hNIS) has also been investigated as a reporter for non-invasive imaging. The physiological role of this transmembrane protein is to actively transport iodine into cells. It is primarily found in thyroid tissue but is also expressed in the salivary glands, the stomach and lactating mammary glands (Penheiter et al., 2012). As hNIS can also promote intracellular accumulation of radiolabelled iodine and anions such as ${ }^{99} \mathrm{TcO}_{4}{ }^{-}$, it has been investigated as both a therapeutic and reporter protein. In a therapeutic context it promotes uptake of ${ }^{131} \mathrm{I}$ and this uptake by endogenous hNIS is 
exploited as a standard treatment for thyroid cancer (Spitzweg et al., 2001). Exogenously over-expressed hNIS $/{ }^{131} \mathrm{I}$ has undergone investigation as a suicide enzyme/prodrug pair to treat a range of cancers including prostate (Barton et al., 2011; Msaouel et al., 2009b), hepatocellular carcinoma (Klutz et al., 2011) and pancreatic cancer (Haddad et al., 2012). PET imaging can be used to detect hNIS expression as it can promote the accumulation of ${ }^{124}$ I. However more commonly hNIS expression is detected by SPECT imaging of ${ }^{99} \mathrm{TcO}_{4}{ }^{-}$accumulation, as arguably the greatest advantage of hNIS as a reporter gene is the ready availability and low cost of ${ }^{99}{ }^{9} \mathrm{TcO}_{4}{ }^{-}$(Hicks and Hofman, 2012).

The clinical use of hNIS as a non-invasive imaging reporter was first described by Barton et al. (Barton et al., 2008) who used it to image a replication competent adenovirus armed with the suicide genes $\mathrm{yCD}$ and $m u t \mathrm{TK}_{\mathrm{SR} 39}$. Prostate cancer patients were administered the virus via intraprostatic injection and then dosed with $\mathrm{Na}^{99 m} \mathrm{TcO}_{4}$. SPECT imaging could detect hNIS expression in 7 out of 9 patients who received the trial's highest dose of $1 \times 10^{12}$ virus particles, with the mean pixel intensity of the SPECT image of injected areas on average 2.3 fold greater than in the uninjected prostate. Repeated imaging was sufficiently sensitive to determine that hNIS expression peaked 1-2 days post virus injection, was confined to the prostate region and that the initial $1 \mathrm{~cm}^{3}$ adenovirus deposits spread to an average of $6.6 \mathrm{~cm}^{3}$, but within too short a timeframe to be due to intratumoural viral replication. A separate research group based out of the Mayo clinic have armed an oncolytic measles virus with $h N I S$ and are currently conducting a Phase I trial (http://www.clinicaltrials.gov/ct2/show/NCT00450814) for treatment of multiple myeloma in which they aim to use repeated SPECT/CT imaging of ${ }^{123}$ I uptake to determine the bio-distribution and longevity the therapeutic virus (Msaouel et al., 2009b). There has also been one report of unsuccessful non-invasive imaging using hNIS, in which a patient treated with intratumoural injection of an adenovirus expressing hNIS $\left(\mathrm{Ad}_{5} / 3-24\right.$-hNIS) did not show SPECT imageable tumour accumulation of either ${ }^{123} \mathrm{I}$ or ${ }^{99 \mathrm{~m}}$ Tc. However, it was suggested that this may have been due to the rapidly acting oncolytic virus lysing infected cells before sufficient intracellular accumulation of radioprobe could occur; pointing to a need to select compatible vector and reporter gene/probe combinations (Rajecki et al., 2011). 


\subsection{Non-invasive imaging of bacterial nitroreductase enzymes}

\subsubsection{Optical imaging}

While nuclear imaging is better suited to clinical applications, the use of significantly more accessible optical imaging technology facilitates the preclinical development of nitroreductase based therapies. Two different quenched fluorescent reporter probes have been used for in vivo imaging of E. coli NfsB. One is 6-chloro-9-nitro-5-oxo-5Hbenzo (a) phenoxazine (CNOB), which on nitroreduction is converted to a fluorescent aminophenoxazin with an emission wave length of $525 \mathrm{~nm}$ that can be detected in small animals (Thorne et al., 2009). When mice carrying $\mathrm{SiHa}$ tumours were treated with Clostridium sporogenes expressing NfsB and co-injected with $\mathrm{CNOB}$, red fluorescence emitted from some regions of the tumour, with particular localisation around the central necrotic core (Liu et al., 2008a). The second compound is CytoCy5S, a quenched fluorophore commercially available from GE Healthcare. This is a freely cell permeable compound, which on nitroreduction is converted to a cell entrapped form that emits a redshifted fluorescent signal (McCormack et al., 2012). CytoCy5S has been shown to noninvasively detect stable expression of $\mathrm{NfsB}$ from multiple different cancer cell lines in mice xenograft models, not only by systemic injection of CytoCy5S, but also by preincubation of the tumour-NfsB cells prior to implantation, as in intact cells the CytoCy5S signal persists post-activation for a number of days (Bhaumik et al., 2011). CytoCy5S also allowed repeated in vivo monitoring of a HSV1-TK-NfsB fusion protein stably expressed from a breast cancer cell line xenograft as part of a GCV/CB1954 tumour growth delay study (Sekar et al., 2012).

\subsubsection{Nuclear imaging}

No bacterial NTR reporter probes suitable for nuclear imaging have previously been described, rendering non-invasive imaging via this modality currently unfeasible. One indirect way through which NTR based GDEPT could be imaged is through a linked gene strategy in which the tumour tropic vector would co-express a second reporter protein (e.g. HSV1-TK, which could report on levels and location of the therapeutic NTR). In such strategies the reporter and therapeutic gene are generally expressed from a bicistronic vector in a single promoter driven construct, which through the use of an internal ribosome entry site allows co-expression of two separate protein products (Tjuvajev et al., 1999). While not unworkable, such imaging would make the output 
signal independent of the presence of the functional NTR protein product, and would require careful experimental validation that the activity of the reporter gene is consistently reflective of the NTR expression in all clinical settings (Wang et al., 2005). This potential disconnect between levels of NTR and reporter gene expression could be avoided by fusion of a NTR and HSV1-TK reporter gene to generate a bi-functional single protein product (such as that generated by (Sekar et al., 2012)); while HSV1-TK based PET imaging of such a construct would again not report directly on NTR function, it would presumably provide a much more reliable indication of expression levels. Moreover, both strategies would have the advantage of enabling synergistic therapeutic effects to be pursued via simultaneous HSV1-TK and NTR based GDEPT (Sekar et al., 2012); and would simplify non-invasive imaging of NTR based therapies as existing imaging modalities and compounds would be used. However it should be noted that there is another possible strategy that would avoid novel compound generation while allowing for direct non-invasive imaging of bacterial NTRs. This could be accomplished by repurposing, as direct NTR reporter probes, PET imaging agents that have already been independently developed and clinically validated.

\subsubsection{2-Nitroimidazole hypoxia imaging agents}

A class of PET reporter probes wherein cellular accumulation is dependent on reduction of a nitro moiety are already in existence - the 2-nitroimidazole (2-NI) hypoxia imaging agents. 2-NI compounds have been researched in an oncology context for more than three decades. Initial interest focused on use in radiation therapy, investigating their ability to act as oxygen mimetics to sensitize hypoxic tumours to ionizing radiation. Drugs trialled under this application included the prototypical misonidazole (Kogelnik et al., 1978), and its analogues etanidazole and pimonidazole (Bleehen et al., 1991). Unfortunately in large scale human trials the compounds demonstrated dose limiting neurotoxicity and failed to show clear clinical benefit (Wardman, 2007). However this work led to the observation that, following nitro group reduction, these drugs covalently bind to cellular macromolecules, becoming cell entrapped. In vivo this reduction occurs only in the presence of hypoxia (Varghese and Whitmore, 1980). As with the previously discussed hypoxia activated prodrugs, this is because these compounds are substrates for a number of oxygen inhibited (i.e. one-electron transferring) human oxidoreductases (Joseph et al., 1994; Wang et al., 2012). 
This observation suggested that monitoring the intracellular accumulation of 2-NI molecules would enable clinicians to detect tumour hypoxia, an important negative prognostic factor. This would enable identification of patients who would benefit from more aggressive or hypoxia targeted treatments, particularly in a radiotherapy context (Marcu et al., 2012; Mees et al., 2009). The use of 2-NI compounds as reporters for clinical non-invasive imaging of hypoxia has most commonly been pursued through addition of an ${ }^{18} \mathrm{~F}$ radioisotope to the 2-NI to enable PET detection. Many of the compounds used are radiolabelled analogues of the initial radiosensitizers and include $\left[{ }^{18} \mathrm{~F}\right] \mathrm{F}-\mathrm{misonidazole}$ (Rasey et al., 1987) and the etanidazole analogues $\left[{ }^{18} \mathrm{~F}\right] \mathrm{EF} 5$ (Komar et al., 2008) and $\left[{ }^{18} \mathrm{~F}\right] \mathrm{EF} 3$ (Mahy et al., 2008). Others such as $\left[{ }^{18} \mathrm{~F}\right]$ FETNIM (Ganjoo et al., 2011), $\left[{ }^{18} \mathrm{~F}\right] \mathrm{HX} 4$ (Loon et al., 2010) and $\left[{ }^{18} \mathrm{~F}\right] \mathrm{FAZA}$ (Mortensen et al., 2012) include novel side groups. The chemical structures of these compounds can be seen in Figure 1-8. Multiple clinical trials in head and neck cancers have demonstrated that using such agents to non-invasively determine tumour oxygenation status provides a prognostic indicator of patient outcome (Mortensen et al., 2012; Rajendran et al., 2006; Rischin et al., 2006; Thorwarth et al., 2005). 
<smiles>O=[N+]([O-])c1nccn1CC(O)C[18F]</smiles>

[18F]F-misonidazole<smiles>O=C(Cn1ccnc1[N+](=O)[O-])NCCC(F)(F)F</smiles>

$\left[{ }^{18} \mathrm{~F}\right] \mathrm{EF} 3$<smiles>O=C(Cn1ccnc1[N+](=O)[O-])NCC(F)(F)C(F)(F)C(F)(F)F</smiles>

$\left.{ }^{18} \mathrm{~F}\right] \mathrm{EF} 5$<smiles>O=[N+]([O-])c1nccn1C[C@H](O)[C@@H](O)C[18F]</smiles>

${ }^{[18}$ F]FETNIM<smiles>O=[N+]([O-])c1nccn1[C@@H]1O[C@H](C[18F])[C@@H](O)[C@H]1O</smiles>

$\left.{ }^{18} \mathrm{~F}\right] \mathrm{FAZA}$<smiles>O=[N+]([O-])c1nccn1Cc1cn(C(CO)C[18F])nn1</smiles>

$\left[{ }^{18} \mathrm{~F}\right] \mathrm{HX} 4$

Figure 1-8 Structures of ${ }^{18} \mathrm{~F}$ 2-NI hypoxia imaging agents with prior human use.

Radiolabelled misonidazole derivatives were the first 2-NI compounds investigated for detection of in vivo hypoxia (Jerabek et al., 1986; Rasey et al., 1987). [ $\left.{ }^{18} \mathrm{~F}\right] \mathrm{F}-$ misonidazole has been the most widely used 2-NI PET imaging agent (Marcu et al., 2012), and along with $\left[{ }^{18} \mathrm{~F}\right] \mathrm{FAZA}$ is included in a International Atomic Energy Agency (IAEA) coordinated research project (CRP No. F22047) aimed at expanding and supporting the use of ${ }^{18} \mathrm{~F}$ radiopharmaceuticals (Coenen et al., 2010). Importantly $\left[{ }^{18} \mathrm{~F}\right] \mathrm{F}$-misonidazole can be can be labelled using nucleophilic fluorination (Lim and Berridge, 1993), a preferred radioisotope labelling method that is procedurally similar to preparing $\left[{ }^{18} \mathrm{~F}\right]-\mathrm{FDG}$, making it easier to adapt $\left[{ }^{18}\right.$ F]FDG existing technology at most medical cyclotrons (Coenen et al., 2010). However, as is evidenced by the number of other 2-NI analogues that have also been developed, $\left[{ }^{18} \mathrm{~F}\right] \mathrm{F}$-misonidazole has a number of features which could be improved 
upon, such as high liver uptake and a relatively high lipophilicity which contributes to slow clearance time and a modest uptake ratio between hypoxic tumours and comparative tissue (blood and/or muscle) (Carlin and Humm, 2012; Padhani, 2006). F-misonidazole signal attenuation has also been suggested to occur due to non-specific in vivo break down of the parental compound (Rasey et al., 1999).

More hydrophilic second generation 2-NIs such $\left[{ }^{18} \mathrm{~F}\right] \mathrm{FAZA}$ or $\left[{ }^{18} \mathrm{~F}\right] \mathrm{HX} 4$ have been designed to promote faster clearance. One $\left[{ }^{18} \mathrm{~F}\right] \mathrm{HX} 4$ human biodistribution study reported low liver uptake, that over a $3.6 \mathrm{~h}$ period $\sim 45 \%$ of the injected activity of ${ }^{18} \mathrm{~F}-\mathrm{HX} 4$ was excreted in the urine, and that $\left[{ }^{18} \mathrm{~F}\right] \mathrm{HX} 4$ remained essentially intact in the blood (Doss et al., 2010). A click chemistry based synthesis and nucleophilic radiolabelling of $\left[{ }^{18} \mathrm{~F}\right] \mathrm{HX} 4$ makes generation of the PET-ready molecule relatively straightforward (Loon et al., 2010). An alternative design strategy is that seen with $\left[{ }^{18} \mathrm{~F}\right] \mathrm{EF}$, where the lipophilicity of the compound has been purposely increased to instead provide high and near uniform tissue penetration and thus stable radiobiological background over the imaging period, $\left[{ }^{18} \mathrm{~F}\right]$ EF5 being chemically stable in vivo (Koch et al., 2010). The electrophilic ${ }^{18} \mathrm{~F}$ fluorination of EF5 is more challenging than nucleophilic displacement (Koch et al., 2010), however research to simplify synthesis and increase radiolabelled yields is ongoing (Chitneni et al., 2012) and could encourage wider use of the compound (Carlin and Humm, 2012).

It should be noted that, when assigning hypoxic status, both $\left[{ }^{18} \mathrm{~F}\right] \mathrm{F}-$ misonidazole and second generation hypoxia imaging agents typically report tumour/blood or tumour/muscle ratios of between 1.3 - 2 (Komar et al., 2008; Loon et al., 2010; Mortensen et al., 2012; Rajendran et al., 2006). Given these fairly modest hypoxia/normoxia ratios it is not implausible to propose that, if bacterial NTRs demonstrate efficient metabolism of these 2-NI compounds, then repurposing of these agents for effective in vivo imaging of bacterial NTR expression may be possible.

\subsection{Aims of this study}

The overall goal of this thesis was to progress the ability to noninvasively image NTR based oncolytic therapies.

The first aim was to interrogate the abilities of a range of naturally occurring bacterial NTRs to reduce 2-NI compounds, radiolabelled forms of which were originally developed 
for noninvasive PET imaging of hypoxia. To achieve this, microplate screens were developed to enable simple and accurate detection of 2-NI reduction by NTR candidates over-expressed in E. coli. This aim was investigated in Chapters 3 and 4. Results generated by our collaborators to assess whether this 2-NI activity could be used to detect in vivo NTR expression in preclinical models are also discussed.

A second aim was to apply the microplate screening methods in a directed evolution project to improve native levels of 2-NI reduction by a lead NTR candidate. This was investigated in Chapter 5.

As an alternative to re-purposing existing (2-NI) PET probes, a third aim of this research was to identify NTRs that can efficiently activate a novel PET suitable compound, designed to be less responsive to hypoxia. An important outcome of this work was the identification of key mutations that can be introduced into variants of E. coli NfsB to improve metabolism of this novel probe molecule. This work is presented in Chapter 6.

Finally, it was aimed that these and other screens would be used in a collaborative directed evolution project, to generate an NTR with improved ability to activate the nitroaromatic DNA damaging prodrug PR-104A, while retaining an ability to reduce 2-NI compounds. This was to generate evolved NTR(s) to progress for further testing in a clinically relevant tumour targeting Clostridium strain. This work is presented in Chapter 7. 


\section{Chapter 2: Methods}

\subsection{Chemical reagents and enzymes}

The compounds PR-104A, RB6154, EF5, SN33623 and all NTR fluorogenic probes were synthesised by various chemists at the Auckland Cancer Society Research Centre and provided to this study by our collaborators Dr. Adam Patterson and Dr. Jeff Smaill (ACSRC; University of Auckland, New Zealand). All compounds were characterised by ${ }^{1} \mathrm{H}$ NMR and mass spectrometry. HX4 was a gift from Prof. Philippe Lambin at the University of Maastricht. EF3 was a gift from Prof. Cameron Koch at the University of Pennsylvania. Pimonidazole was purchased from Bioscience Research Reagents (Temecula, CA, USA) and F-misonidazole from ABX Company (Chemical Division, Radeberg, Germany). Unless otherwise stated, stock solutions of all drug and imaging agent compounds were made up in DMSO and stored at $-80{ }^{\circ} \mathrm{C}$. Restriction enzymes were purchased from New England Biolabs (NEB; Ipswich, MA, USA). BioMix ${ }^{\mathrm{TM}}$ Red Polymerase Mastermix was purchased from Bioline (London, UK). Phusion ${ }^{\mathrm{TM}}$ highfidelity DNA polymerase was purchased from Finnzymes (Espoo, Finland). Unless otherwise stated all other chemicals and reagents were purchased from Sigma-Aldrich (St. Louis. MO, USA) or Thermo Fisher Scientific (Waltham, MA, USA).

\subsection{Oligonucleotide primers}

Oligonucleotide primers used in this study were supplied as lyophilised powders by Invitrogen (Carlsbad, CA, USA) or Integrated DNA Technologies (IDT; Coralville, IA, USA). Stock solutions of $100 \mu \mathrm{M}$ were made up in TE (10 mM Tris-Cl pH 8.0, 0.1 mM EDTA) and $10 \mu \mathrm{M}$ working solutions were diluted in autoclaved and filter sterilized ddH${ }_{2} \mathrm{O}$. Working and stock solutions were stored at $-20{ }^{\circ} \mathrm{C}$. The sequences of all primers used in this study are listed in Table 1-1. 


\begin{tabular}{|c|c|}
\hline Primer & Sequence $\left(5^{\prime} \rightarrow 3^{\prime}\right)$ \\
\hline \multicolumn{2}{|l|}{ NTR specific primer } \\
\hline NfsA_Ec_Fw & GGCATATGACGCCAACCATTGAAC \\
\hline NfsA_Ec_Rv & GGGTCGACTTAGCGCGTCGCCCAACCCTG \\
\hline NfsA_St_Fw & GGGGCATATGAGTCCGACCATTGAA \\
\hline NfsA_St_Rv & GGGGTCGACTTAGCGCGTCGCCCAGCC \\
\hline NfsA_Ck_Fw & GGGGCATATGACTCCAACCATTGATTT \\
\hline NfsA_Ck_Rv & GGGGGTCGACTTAGCGCGTCGCCCAA \\
\hline NfsA_Kp_Fw & GGGGCATATGACGCCGACCATTGAG \\
\hline NfsA_Kp_Rv & GGGGTCGACTCAGCGCGTTGCCCACC \\
\hline NfsA_Ecaro_Fw & GGGGCATATGATACCAACTATTGATTTGCTACA \\
\hline NfsA_Ecaro_Rv & GGGGCTCGAGCTAGCGTATCGCCCATCCTTGTTG \\
\hline NfsA_Es_Fw & CCCCСATATGACGCCAACGATTGAGCTGC \\
\hline NfsA_Es_Rv & GGGGCTCGAGTTAGCGTGTCGCCCAGCCCTG \\
\hline NfsA_Vf_Fw & CCCCATATGAACCCTGTTATCGATAC \\
\hline NfsA_Vf_Rv & CCCGTCGACTCACTTAATAGCTAGGC \\
\hline NfsA_Vv_Fw & GGGGCATATGAACGCTGTTATTGAC \\
\hline NfsA_Vv_Rv & GGGGTCGACTTACTTTGTCGCCAAGCC \\
\hline Frp_Vh_Fw & GGGCATATGAACAATACGATTGAAA \\
\hline Frp_Vh_Rv & GGGGTCGACTTAGCGTTTTGCTAGCC \\
\hline NfrA_Bs_Fw & GGGGCATATGAATAACACAATCGAAACC \\
\hline NfrA_Bs_Rv & GGGGGTCGACTTAGTTTTTATTAAAGCCCT \\
\hline NfsA_Li_Lw_Fw & GGGGCATATGAATCAGGCGATAGATGCCATTTT \\
\hline NfsA_Li_Lw_Rv & GGGGGTCGACTTATTTTTGATTTAAATGTTGC \\
\hline NfsA_Bc_Fw & GGGGCATATGAATACTATCATTGAAACGATTCTC \\
\hline NfsA_Bc_Rv & GGGGGTCGACTTACTTTTTATCAAACCCTTGGCGT \\
\hline NfsA_0199NP_Fw & GGCATATGCCTTTACAGATGGAA \\
\hline NfsA_0199NP_Rv & 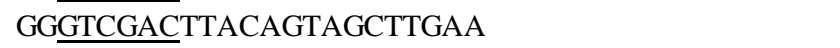 \\
\hline NfsA_Bth_Fw & GGGGCATATGAATGAAATGATACATAAAATGGAG \\
\hline NfsA_Bth_Rv & GGGGGTCGACTCATTTCTTATTTAATCCTCTCTCAT \\
\hline NfsA_Lsak_Fw & GGGGCATATGTCTGATTTAATCGCACAAATGCAAC \\
\hline NfsA_Lsak_Rv & GGGGGTCGACTTATGCTAATGTAAACCCCTGTTTCTT \\
\hline EcD_Pp_Fw & CCCCㅡㅡATATGAGCCTTCAAGACGAAG \\
\hline EcD_Pp_Rv & CTAGGTCGACTCAGCGCAGGCCGAAAC \\
\hline YcnD_Bs_Fw & 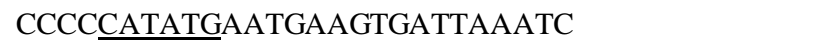 \\
\hline YcnD_Bs_Rv & 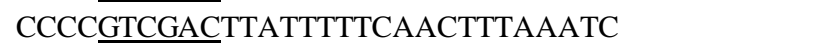 \\
\hline NfsA_Ms_Fw & GGGGCATATGACGGTCATCGCGCGCTACGCAGACGTCGAT \\
\hline NfsA_Ms_Rv & GGGGCTCGAGTCAGCGGATTCCCAGGCCCAACCGCTCG \\
\hline NfsB_Ec_Fw & GGGCATATGGATATCATTTCTG \\
\hline NfsB_Ec_Rv & GGGGAATTCTTACACTTCGGTTAAG \\
\hline NfsB_Ck_Fw & CCCCCATATGGATATCGTTTCTGTCGC \\
\hline NfsB_Ck_Rv & CCCGTCGACTCAGACTTCCGTCAGGGTG \\
\hline NfsB_St_Fw & GGGGCATATGGATATCGTTTCTGTC \\
\hline NfsB_St_Rv & GGGGGTCGACTTAGACTTCCGTCAGTG \\
\hline NfsB_Kp_Fw & GGGGCATATGGATATCGTATCGGTC \\
\hline NfsB_Kp_Rv & GGGGCTCGAGTTAAATCTCGGTAATGGT \\
\hline NfsB_Vv_Fw & GGGGCATATGACTATTGTTCAAGCT \\
\hline NfsB_Vv_Rv & GGGGTCGACTTAGATTTCGGTAAAAACAG \\
\hline 2432_Pp_Fw & GGGGCATATGGATACCGTATCGCTG \\
\hline 2432_Pp_Rv & GGGGCTCGAGTCAGAGGAAGGTGAACACTT \\
\hline NfsB_Es_Fw & CCCC $\overline{\overline{C A T A T G}} A$ ACCTTAATGAGATCATTCGC \\
\hline NfsB_Es_Rv & CCCGTCGACTCAGAGCTGCGTGATCAC \\
\hline FraseI_Vf_Fw & GGGGCATATGACGCATCCAATTATTCA \\
\hline FraseI_Vf_Rv & GGGGGTCGACCTAAAGAATGGTAATTAC \\
\hline NfsB_Vh_Fw & CCССCATATGTCTCATCAAATCATTACAGAC \\
\hline
\end{tabular}




\begin{tabular}{|c|c|}
\hline NfsB_Vh_Rv & CCCCTCGAGTTAAAGAGTAGTAATTACGTC \\
\hline YfkO_Bs_Fw & GGGGCATATGGCAGATCTAAAGACACA \\
\hline YfkO_Bs_Rv & CTAGGTCGACTTAAACCCACTTCACAACAT \\
\hline YdgI_Bs_Fw & GGGGCATATGATCAAAACAAACGATTTTATGG \\
\hline YdgI_Bs_Rv & GGGGGTCGACTTATTTCCATTCTGCAATTG \\
\hline 5190_Pa_Fw & GGGGCATATGCATATCGAAGACGCC \\
\hline 5190_Pa_Rv & GGGGGTCGACTCAGAAGCGGTCGCGAATGA \\
\hline AzoR_Ec_Fw & GGGGCATATGAGCAAGGTATTAGTTCTT \\
\hline AzoR_Ec_Rv & GGGGGTCGACTTATGCAGAAACAATGCTGT \\
\hline AzoR_St_Fw & CCCGCATATGAGCAAGGTATTAGTTCTT \\
\hline AzoR_St_Rv & CCCGTCGACTTAAGCGGCCACGAC \\
\hline AzoR_Vv_Fw & CCCGCATATGTCTCGTTTACTAGTATT \\
\hline AzoR_Vv_Rv & CCCGGTCGACTTAAGCTGTTAGCGCTGCAAG \\
\hline 4538_Pp_Fw & GGGGCATATGTCCCGCGTACTGATCATC \\
\hline 4538_Pp_Rv & GGGGCTCGAGTCAGGCCACCGCTGCCAGCTT \\
\hline NemA_Ec_Fw & CTAGGGATCCATGTCATCTGAAAAACTG \\
\hline NemA_Ec_Rv & CTAGGTCGACTTACAACGTCGGGTAATC \\
\hline NemA_St_Fw & CCCGGATCCATGTCATCAGCAAAACTG \\
\hline NemA_St_Rv & CCCGGTCGACTTACAGAGTAGGGTAGTC \\
\hline NemA_Kp_Fw & CCCCATATGTCGGAAGCAA \\
\hline NemA_Kp_Rv & CCCCTCGAGTTACAGAGTCGGGTAA \\
\hline NemA_Vv_Fw & CCCCATATGAGCAAACTGTTTGAA \\
\hline NemA_Vv_Rv & CCCGTCGACTCACACATGCAAAGCGGT \\
\hline YwrO_Bs_Fw & 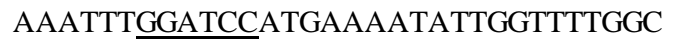 \\
\hline YwrO_Bs_Rv & CCCGTCGACTTAAACAAAAGGCTGCTG \\
\hline YwrO_Li_Fw & CCCCCATATGAAAACATTAGTTATTAT \\
\hline YwrO_Li_Rv & CCCGTCGACCTAATTTAACGTTTTAATA \\
\hline YwrO_Vf_Fw & AAATTTGGATCCATGACAAGCCCGGCT \\
\hline YwrO_Vf_Rv & CCCGTCGACTTATTCCTTAATAAATTTATC \\
\hline YieF_Ec_Fw & GGGGCATATGTCTGAAAAATTGCAGGTG \\
\hline YieF_Ec_Rv & GGGGGTCGACTTAGATCTTAACTCGCTG \\
\hline 1204_Pa_Fw & GGGGCATATGAGCGACGACATCAAG \\
\hline 1204_Pa_Rv & GGGGGTCGACTCAACCGCGCAGGCGGCGCA \\
\hline MdaB_Ec_Fw & GGGCATATGAGCAACATCCTG \\
\hline MdaB_Ec_Rv & GGGGTCGACTTAACCAAAAATTTC \\
\hline MdaB_Ps_Fw & GGGGCATATGAAAAAAGTATTGTTCCTCAAC \\
\hline MdaB_Ps_Rv & CTAGCTCGAGTTACTGCGCACCAAACACTT \\
\hline WrbA_Ec_Fw & GGGCATATGGCTAAAGTTCTGGTG \\
\hline WrbA_Ec_Rv & GGGGTCGACTTAGCCGTTAAGTTT \\
\hline WrbA_Ps_Fw & GGGGCATATGAGCAAACCTTACATTCTGG \\
\hline WrbA_Ps_Rv & CTAGGTCGACTCAGCTGCGTGTTGTGTC \\
\hline YdjA_Ec_Fw & CTAGCATATGGATGCACTCGAACTATTG \\
\hline YdjA_Ec_Rv & CTAGGTCGACTCAGAAATAAGTTACAAACGG \\
\hline YdjA_Kp_Fw & CCCC스ATATGATGCTCTCGATCTACTGC \\
\hline YdjA_Kp_Rv & CCCCGTCGACTCAGAATCGCGTCACAAACG \\
\hline 3720_Pp_Fw & GGGGCATATGCTTGTGAATGTACTG \\
\hline 3720_Pp_Rv & GGGGGTCGACTCACGTCAGCGGGTACAACG \\
\hline 4975_Pa_Fw & GGGGCATATGAACGTACTGATCGTC \\
\hline 4975_Pa_Rv & GGGGGTCGACTCAGCGCGCCAGCGGCTGGA \\
\hline YcdI_Ec_Fw & CTAGCATATGAACGAAGCCGTTAGCC \\
\hline YcdI_Ec_Rv & CTAGGTCGACTTACAACAGCCCGCAGG \\
\hline YcdI_Kp_Fw & GGGGCATATGAACGACGCGATAAACCA \\
\hline YcdI_Kp_Rv & CTAGCTCGAGTTATGCCAGGCAGGCT \\
\hline YcaK_Ec_Fw & GGGGCATATGCAGTCTGAACGTATT \\
\hline YcaK_Ec_Rv & GGGGGTCGACTTATAGTGCATCTACCATAT \\
\hline
\end{tabular}




\begin{tabular}{ll}
\hline 0853_Pa_Fw & GGGGCATATGGTTGAAACCGCCAAGACC \\
0853_Pa_Rv & GGGGGTCGACCTAGACCTGGCTGCCGAGCT \\
KefF_Ec_Fw & GGGGCATATGATTCTTATAATTTATGCG \\
KefF_Ec_Rv & GCCTGA $\underline{\text { GTCGACGTATGGCTATCCATGATGGG }}$
\end{tabular}

Primers used in constructing NfsA_Ec site saturation mutagenisis libraries

NfsAEC_40_NNKFwd

NfsAEC_40_41_Rv

NfsAEC_41_NNKFwd

NfsAEC_42_NNKFwd

NfsAEC_42_43_Rv

NfsAEC_43_NNKFwd

NfsAEC_45_NNKFwd

NfsAEC_45_Rv

NfsAEC_83_NNKFwd

NfsAEC_83_Rv

NfsAEC_103_NNKFwd

NfsAEC_103_Rv

NfsAEC_107_NNKFwd

NfsAEC_107_Rv

NfsAEC_134_NNKFwd

NfsAEC_134_Rv

NfsAEC_215_NNKFwd

NfsAEC_215_Rv

NfsAEC_224_NNKFwd

NfsAEC_224_Rv

NfsAEC_225_Rv

NfsA EC_41_45 NNK
GCGCCCGTGCGACGTCCAGTNNKAGTTTTTTGCAGTGC

ACTGGACGTCGCACGGGC

GCCCGTGCGACGTCCAGTTCCNNKTTTTTGCAGTGCAGTAG

GTGCGACGTCCAGTTCCAGTNNKTTGCAGTGCAGTAGCATT

ACTGGAACTGGACGTCGCAC

GTGCGACGTCCAGTTCCAGTTTTNNKCAGTGCAGTAGCATTC

CCAGTTCCAGTTTTTTGCAGNNKAGTAGCATTATTCGCAT

CTGCAAAAAACTGGAACTGG

TCTGGGTGTTCTGTGCCGACNNKAACCGCCATTTACAGATC

GTCGGCACAGAACACCCAGA

GCCTGGCGGAACAACTGTTGNNKGGTGTCGTTGATACG

CAACAGTTGTTCCGCCAGGC

AACTGTTGCTCGGTGTCGTTNNKCGGCAATGATGGCGCAG

AACGACACCGAGCAACAGTT

GTATATATCGGCGGCCTGCGCNNKAATATTGAAGCGGTGACG

GCGCAGGCCGCCGATATATACC

GCCGGGATACCTGGAGCGATNNKATCCGCCGAACAATCATT

ATCGCTCCAGGTATCCCGGC

GCCGAACAATCATTAAAGAANNKCGCCCATTTATTCTGGAT

TTCTTTAATGATTGTTCGGC

CCGTCGACTTAGCGCGTCGCCCAACCCTGTTTGTGCAAATAATCCAGAATAAATGGMNN GCTTTCTTTA

GCCCGTGCGACGTCCAGTTCCNNKTTTTTGCAGNNKAGTAGCATTATTCG

\section{Primers used in construction of Nfs A_Ec multi-site mutant library}

Reverse primers not shown were identical to those used in construction of site saturation mutagenisis libraries Combo 12 Fwd GGGGTATCATATGACGCCAACCATTGAACTTATTTGTGGCCATMGCTCCATTCGCC

Combo_40_41_42_fwd GCCCGTGCGACGTCCAGTWCTTVTWTTTTGCAGTGCAGTAGCATTATTCG

Combo_40_41_42_45_fwd GCCCGTGCGACGTCCAGTWCTTVTWTTTTGCAGWTGAGTAGCATTATTCG

Combo_40_41_42_45_rev CAACAGTTGTTCCGCCAGG

Combo_103_fwd CCTGGCGGAACAACTGTTGSTGGGTGTCGTTGATACGG

Combo_134_wt_fwd

Combo_134_A_fwd

GGTATATATCGGCGGCCTGCGCAATAATATTGAAGCGGTGACG GGTATATATCGGCGGCCTGCGCGCGAATATTGAAGCGGTGACG

Combo_215_fwd CGGGATACCTGGAGCGATCRTATCCGCCGAACAATCATT

Primers used in construction of Nfs $B \_E c$ and Nfs B_Es site specific mutants

NfsBesak67A_F Fwd NfsBesak67A_F Rev NfsBesak69Q_E Fwd NfsB EsakY105F Fwd NfsB EsakY105F Rev NfsBesak125N_H Fwd NfsBesak125N_H Rev NfsB EC_T41L fwd NfsB EC_T41L rev NfsBEC_F70A Fwd NfsBEC_F70A Rev NfsB EC F70A_N71S NfsB EC_N71S TCCTGCCGGCGCTGATGGACTTCAACCAGGTAAAAGCGA GTCCATCAGCGCCGGCAGGA TCCTGCCGGCGCTGATGGACGCCAACGAGGTAAAAGCGAAA GAGCAGCAGGATGGTCGCTTTGACGGCGAGGAAGCGCG GCGACCATCCTGCTGCTC CCGCTTTTTTGTGGGTCTGCACAGCGAAACGCCGGAGCA CAGACCCACAAAAAAGCGG CAATACAGCCCATCCAGCCTGAACTCCCAGCCGTGGC GCTGGATGGGCTGTATTG CGCTGCCGGTAATTACGTGGCCAACGAGCGTAAAATG CACGTAATTACCGGCAGCG CGCTGCCGGTAATTACGTGGCCAGCGAGCGTAAAATGCT CGCTGCCGGTAATTACGTGTTCAGCGAGCGTAAAATGCT 


\begin{tabular}{ll}
\hline NfsBEC_E72Q & CCGGTAATTACGTGTTCAACCAGCGTAAAATGCTTGAT \\
NfsBEC_F70A_72Q & CGCTGCCGGTAATTACGTGGCCAACCAGCGTAAAATGCTTG \\
NfsB EC_T108Y Fwd & GAAGATGCCGATGGCCGCTACGCCACGCCGGAAGC \\
NfsB EC_T108Y Rev & GCGGCCATCGGCATCTTC
\end{tabular}

Primers used in construction of Gate way constructs

NfsA_Ec_GW_Fw GGGGACAAGTTTGTACAAAAAAGCAGGCTTCGAAGGAGATAGAACCATGGGCACG CCAACCATTGAACT

NfsA_Ec_GW_Rv GGGGACCACTTTGTACAAGAAAGCTGGGTCCTAGCGCGTCGCCCAACCCTG

NfsB_Ec_GW_Fwd GGGGACAAGTTTGTACAAAAAAGCAGGCTTCGAAGGAGATAGAACCATGGGCGAT ATCATTTCTGTCGCCTT

NfsB_Ec_GW_Rev GGGGACCACTTTGTACAAGAAAGCTGGGTCCTACACTTCGGTTAAGGTGATGT

NfsB_Esak_GW_Fwd GGGGACAAGTTTGTACAAAAAAGCAGGCTTCGAAGGAGATAGAACCATGGGCAAC CTTAATGAGATCATTCG

NfsB_Esak_GW_Rev GGGGACCACTTTGTACAAGAAAGCTGGGTCCTAGAGCTGCGTGATCACCACAT

Primers used for gene knock out

$\begin{array}{ll}\text { TolC_KO_Fw } & \text { ATCGGCCTGAGCCTTTCTGGGTTCAGTTCGTTGAGCTGTGTAGGCTGGAGCTGCTTC } \\ \text { TolC_KO_ext_Fw } & \text { ATGAAGAAATTGCTCCCCATTCTTATCGGCCTGAGCCTT } \\ \text { TolC_KO_Rv } & \text { GTTACTGGTGGTAGTGCGTGCGGATGTTGCTGAACCATATGAATATCCTCCTTAG } \\ \text { TolC_KO_ext_Rv } & \text { TCAGTTACGGAAAGGGTTATGACCGTTACTGGTGGTAGT } \\ \text { NfsA_KO_Fw } & \text { GGGCAGAAAGAGAAAAAGATAATGACGCCAACCATTGAATGTGTAGGCTGGAGCT } \\ & \text { GCTTC } \\ \text { NfsA_KO_Rv } & \text { GGGTACATCGACGTGGCGGTTTTAGCGCGTCGCCCAACCCATATGAATATCCTCCTTAG } \\ \text { NfsB_KO_Fw } & \text { GGGCCCGGCAAGAGAGAATTACACTTCGGTTAAGGTGATTGTGTAGGCTGGAGCTGC } \\ & \text { TTC } \\ \text { NfsB_KO_Rv } & \text { GGGTCACATGGAGTCTTTATGGATATCATTTCTGTCGCCCATATGAATATCCTCCTTAG } \\ \text { AzoR_KO_Fw } & \text { TTATGCAGAAACAATGCTGTCGATGGCTGCTTTTGCTGTGTAGGCTGGAGCTGCTTG } \\ \text { AzoR_KO_ext_Fw } & \text { CAAACATCTATAAGGAAACACCATGAGCAAGGTATTAGTTC } \\ \text { AzoR_KO_Rv } & \text { ATGAGCAAGGTATTAGTTCTTAAATCCAGCATCCTGCATATGAATATCCTCCTTAG } \\ \text { AzoR_KO_ext_Rv } & \text { CCACATCAAGACCGTGTCCGGTTATGCAGAAACAATGCTG } \\ \text { NemA_KO_Fw } & \text { ATGTCATCTGAAAAACTGTATTCCCCACTGAAAGTGTGTGTAGGCTGGAGCTGCTTC } \\ \text { NemA_KO_ext_Fw } & \text { ATCACCAGACGACCGGGAGCCTTTATGTCATCTGAAAAACGT } \\ \text { NemA_KO_Rv } & \text { TTACAACGTCGGGTAATCGGTATAGCCTTCCGCGCCCATATGAATATCCTCCTTAG } \\ \text { NemA_KOext_Rv } & \text { CTTTACGCCGCTCGCAATGTTGGATTACAACGTCGGGTAAT }\end{array}$

Miscellaneous primers

pMMBFw GGCTCGTATAATGTGTGG

pMMBRv $\quad$ GACCGCTTCTGCGTTCTGAT

T7prom TAATACGACTCACTATAGGG

T7term GCTAGTTATTGCTCAGCGG

M13Fw GTAAAACGACGGCCAG

M13Rv CAGGAAACAGCTATGAC

Underlined text indicates restriction sites

Table 2-1 Primers used in this study 


\subsection{Strains, cell lines and plasmids used in this study}

\subsubsection{Bacterial strains}

All bacterial strains used in this study are described in Table 2-2 and Table 2-3.

\begin{tabular}{|c|c|c|}
\hline Strain & Relevant characteristics & Source \\
\hline DH5a & supE 44 DlacU 169 (Ø80 lacZ DM5) hsdR 17 & Invitrogen \\
\hline BL21 & $\mathrm{F}^{-}$ompT gal dcm lon hsdS $\mathrm{B}_{\mathrm{B}}\left(\mathrm{r}_{\mathrm{B}}^{-} \mathrm{m}_{\mathrm{B}}^{-}\right) \lambda(\mathrm{DE} 3)$ & Novagen \\
\hline ADA-510 & F lac-6 (del) $\quad($ sfiA::lacZ $)$ & (Shapiro and Baneyx 2002) \\
\hline SOS-R1 & ADA-510 $\Delta n f_{s} A \Delta n f_{s} B$ & (Prosser et al., 2010) \\
\hline SOS-R2 & SOS-R1 $\Delta$ azoR $\Delta$ nemA $\Delta$ tolC & (Prosser et al., 2013) \\
\hline W3110 & $\mathrm{F}-\lambda-\mathrm{IN}(\mathrm{rrnD}-\mathrm{rrnE}) 1 \mathrm{rph} 1$ & Lab stock \\
\hline $6 \mathrm{KO}$ & W3110 $\Delta n f s A \Delta n f s B \Delta y i e F \Delta y c a K \Delta m d a B \Delta a z o R$ & (Horvat, 2012) \\
\hline $7 \mathrm{KO}$ & $6 \mathrm{KO} \Delta$ nemA & This study \\
\hline
\end{tabular}

Table 2-2 Escherichia coli strains used in the study

Strains used as a source of bacterial oxidoreductase enzymes in this study

Lab stocks:

Escherichia coli $\mathrm{W} 3110$

Pseudomonas aeruginosa $\mathrm{PAO} 1$

Pseudomonas putida KT2440

Pseudomonas syringae pv. phaseolicola 1448a

Mycobacterium smegmatis str. $\mathrm{MC}^{2} 155$

ESR culture collection*:

Salmonella enterica (typhimurium) Ty2 ATCC19430

Klebsiella pneumoniae ATCC 13883

Vibrio vulnificus ATCC $27562 * *$

Vibrio harveyi ATCC $33843 * *$

Vibrio harveyi KCTC $2720 * *$

Vibrio fischeri ATCC7744**

Bacillus subtilis subsp. subtilis NZ isolate

Enterobacter (Chronobacter) sakazaki ATCC 29544

Citrobacter koseri ATCC 27156

Listeria innocua Clip11262

Bacillus coagulans ATCC 7050

Listeria welshimeri ATCC 35897

Lactobacillus sakei subsp. sakei $23 \mathrm{~K}$

Erwinia carotovora subsp. Atrosepticum SCRI1043

Bacillus thuringiensis serovar konkukian str. 97-27

Nostoc punctiforme PCC 73102

* Obtained from Institute of Environmental Science and Research, Porirua, NZ

** propagated in Marine Broth

Table 2-3 Strains used as sources of oxidoreductases in expanded NTR candidate library 


\subsubsection{Mammalian cell line}

HCT-116, an adherent colorectal carcinoma cell line, was purchased from ATCC (American Type Culture Collection, Manassas, VA, USA).

\subsubsection{Plasmids}

All plasmids used for transformation of bacterial and mammalian cells are listed in Table 2-4. Also shown are plasmid maps of the vectors used for expression of bacterial oxidoreductase enzymes, both from E. coli (pUCX- Figure 2-1) and HCT-116 (F279-V5-

Figure 2-2).

\begin{tabular}{|c|c|c|}
\hline Plasmid & Relevant characteristics & Source \\
\hline $\mathrm{pUCX}$ & $\mathrm{Amp}^{\mathrm{R}}$ E. coli expression vector. tac promoter, lac operator, $\mathrm{pET} 28 \mathrm{~A}(+) \mathrm{RBS}$. & (Prosser et al., 2010) \\
\hline pET28A $(+)$ & $\mathrm{Kan}^{\mathrm{R}}$ Expression vector for His6-tagged enzyme expression. $\mathrm{T} 7$ promoter. & Novagen \\
\hline pGFP & $\begin{array}{l}\mathrm{Spec}^{\mathrm{R}} \mathrm{Chl}^{\mathrm{R},} \text { pCloDF13 origin of replication, expresses } g f p \text {-mut } 3 \text { under control of } \\
\text { sulA/sfiA SOS promoter. }\end{array}$ & Dr. Janine Copp, unpublished ${ }^{1}$ \\
\hline pKD4 & $\mathrm{Amp}^{\mathrm{R}} \mathrm{Kan}^{\mathrm{R}}$ Pir-origin of replication. $\mathrm{kan}^{R}$ gene template for KO-PCR & (Datsenko and Wanner, 2000) \\
\hline pKD46 & $\begin{array}{l}\text { Amp }{ }^{R} \text { Heat sensitive origin of replication. Recombinase expression vector. ara } \\
\text { operon promoter. }\end{array}$ & (Datsenko and Wanner, 2000) \\
\hline pCP20 & $A m p^{R}$ Heat sensitive origin of replication. Encodes FLP recombinase. & (Datsenko and Wanner, 2000) \\
\hline pDONR221 & $\mathrm{Kan}^{\mathrm{R}}$ Entry vector for Gateway recombination cloning & Invitrogen \\
\hline F279-V5 & $\begin{array}{l}\text { Destination vector for gateway recombination cloning. Mammalian expression } \\
\text { vector, } \text { Puro }^{R} \text { Amp }^{R} \text { CMV promoter. }\end{array}$ & ACSRC, (Prosser et al., 2013) \\
\hline
\end{tabular}

${ }^{1}$ constructed by PCR-amplifying the sulA/sfiA promoter, gfp mut3 and downstream chloramphenicol cassette from pANO1 (Norman et al., 2005) and cloning this fragment into pCDFDuet-1 (Novagen).

Table 2-4 Plasmids used in this study 


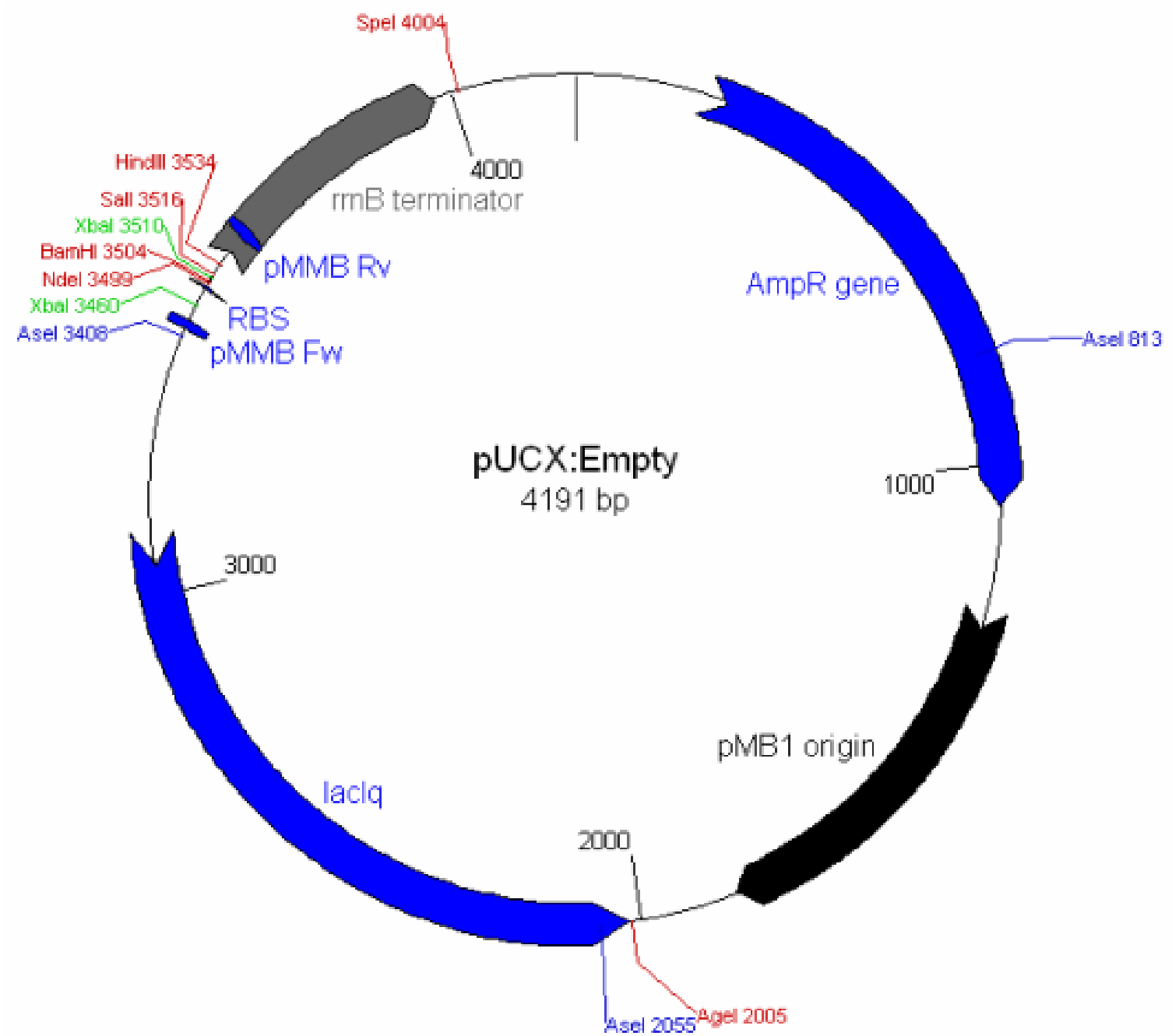

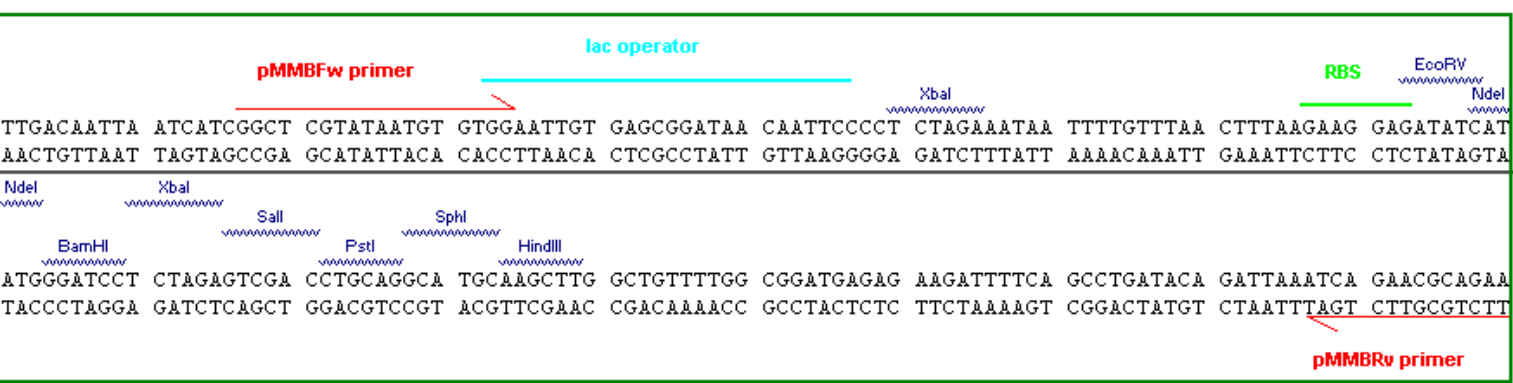

Figure 2-1 Plasmid map and multiple cloning site of $E$. coli expression vector pUCX empty. Post insertion of the DNA sequence of various bacterial NTRs into the multi-cloning site this vector was used for recombinant bacterial NTR expression from E. coli. The plasmid as pictured here was used as the empty plasmid control for all experiments where such a control was needed. 


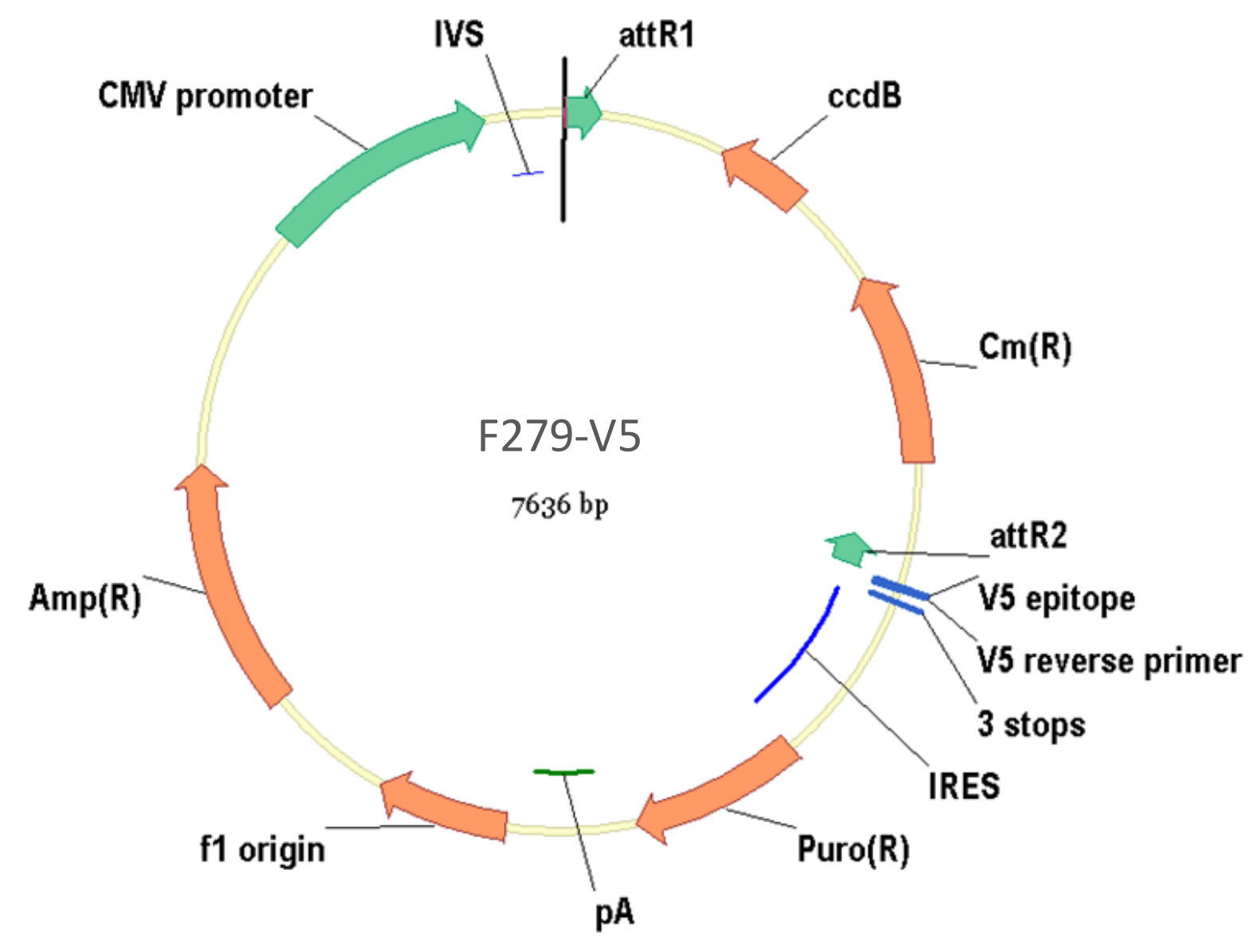

Figure 2-2 Plasmid map of F279-V5, the plasmid vector used for NTR expression from HCT-116 cells. NTR sequences were inserted in-between recombination sites attR1 and attr2 using Gateway® (Invitrogen) cloning.

\subsection{Bacterial growth and maintenance}

\subsubsection{Growth media}

Unless otherwise stated all media were made up in $\mathrm{ddH}_{2} \mathrm{O}$ and sterilised by autoclaving at $121{ }^{\circ} \mathrm{C}$ for $30 \mathrm{~min}$. Media were stored at room temperature, or at $4{ }^{\circ} \mathrm{C}$ if containing heat sensitive reagents.

\subsubsection{Liquid Media}

\section{Luria broth $(\mathbf{L B}) *$}

Tryptone 10 g.L ${ }^{-1}$

Yeast extract 5 g.L $\mathrm{L}^{-1}$

$\mathrm{NaCl} 5$ g.L $\mathrm{L}^{-1}$

*LB was provided as a premixed powder by manufacturer

\section{SOC*}

Tryptone 20 g.L L $^{-1}$

Yeast extract 5 g.L $\mathrm{L}^{-1}$ 
$\mathrm{NaCl} 10 \mathrm{mM}$

$\mathrm{KCl} 2.5 \mathrm{mM}$

$\mathrm{MgCl}_{2} 10 \mathrm{mM} * *$

Glucose $20 \mathrm{mM}^{* *}$

*Adjusted to $\mathrm{pH} 7.0$ using $\mathrm{NaOH}$

**Filter sterilized and added post-autoclave

\section{GYT Medium*}

$10 \%$ glycerol

Yeast extract 1.25 g.L - $^{-1}$

Tryptone 2.5 g.L $\mathrm{L}^{-1}$

*Filter sterilized

\section{Autoinduction media (ZYP-5052)}

ZY base 919 mL.L ${ }^{-1}$

20 x NPS 50 mL.L ${ }^{-1}$

$50 \times 505220 \mathrm{~mL} . \mathrm{L}^{-1}$

$100 \mathrm{x}$ Metal salts $10 \mathrm{ml} . \mathrm{L}^{-1}$

$\mathrm{MgSO}_{4} 1 \mathrm{mM}^{*}$

*filter sterilized and added post autoclave

\section{ZY base}

Tryptone 10 g.L L $^{-1}$

Yeast extract 5 g. $\mathrm{L}^{-1}$

\section{$20 \times$ NPS}

$\mathrm{NH}_{4} \mathrm{SO}_{2} 0.5 \mathrm{M}$

$\mathrm{KH}_{2} \mathrm{PO}_{4} 1 \mathrm{M}$

$\mathrm{Na}_{2} \mathrm{HPO}_{4} 1 \mathrm{M}$

\section{$50 \times 5052$}

Glycerol 250 g.L L $^{-1}$

Glucose 25 g.L ${ }^{-1}$

Lactose 100 g.L ${ }^{-1}$

100x Metal salts

$\mathrm{CaCl}_{2} .2 \mathrm{H}_{2} \mathrm{O} 0.1$ g.L $\mathrm{L}^{-1}$

$\mathrm{CoCl}_{2} .2 \mathrm{H}_{2} \mathrm{O} 0.04$ g. $\mathrm{L}^{-1}$

$\mathrm{CuSO}_{4} .5 \mathrm{H}_{2} \mathrm{O} 0.02$ g.L $\mathrm{L}^{-1}$

EDTA 1 g.L ${ }^{-1}$

$\mathrm{FeSO}_{4} .7 \mathrm{H}_{2} \mathrm{O} 0.5$ g. $\mathrm{L}^{-1}$

$\mathrm{MgCl}_{2} \cdot 6 \mathrm{H}_{2} \mathrm{O} 10 \mathrm{~g} . \mathrm{L}^{-1}$

$\mathrm{MnCl}_{2} .2 \mathrm{H}_{2} \mathrm{O} 0.1$ g. $\mathrm{L}^{-1}$

$\mathrm{NaMoO}_{4} .2 \mathrm{H}_{2} \mathrm{O} 0.02$ g.L $\mathrm{L}^{-1}$

$\mathrm{ZnSO}_{4} .7 \mathrm{H}_{2} \mathrm{O} 0.2$ g. $\mathrm{L}^{-1}$ 


\section{Marine Broth}

Marine broth was supplied by the manufacture (Sigma-Aldrich) as a ready mixed Powder and prepared as per manufacturer's instructions.

\subsubsection{Solid Media}

Agar plates for growth on solid media were made by addition of agar $1.5 \%(\mathrm{w} / \mathrm{v})$ to $\mathrm{LB}$ prior to autoclaving. Any necessary antibiotics were added post autoclave once the media had cooled to $<50{ }^{\circ} \mathrm{C}$.

\subsubsection{Bacterial antibiotics}

Antibiotics were made up in stock solutions at 1000x final concentration (Table 2-5) and filter sterilized using a $0.22 \mu \mathrm{M}$ filter (if made up in $\mathrm{ddH}_{2} \mathrm{O}$ ) and stored in aliquots at -20 ${ }^{\circ} \mathrm{C}$.

\begin{tabular}{lcc}
\hline Antibiotic & Final concentration & Solvent \\
\hline Ampicillin & $100 \mu \mathrm{g} . \mathrm{L}^{-1}$ & $\mathrm{dd} \mathrm{H}_{2} \mathrm{O}$ \\
Kanamycin & $50 \mu \mathrm{g} . \mathrm{L}^{-1}$ & $\mathrm{dd} \mathrm{H}_{2} \mathrm{O}$ \\
Spectinomycin & $50 \mu \mathrm{g} \cdot \mathrm{L}^{-1}$ & $\mathrm{dd} \mathrm{H}_{2} \mathrm{O}$ \\
Chloramphenicol & $34 \mu \mathrm{g} \cdot \mathrm{L}^{-1}$ & $100 \%$ ethanol \\
\hline
\end{tabular}

Table 2-5 Antibiotic concentrations

\subsubsection{Growth and storage}

Unless otherwise stated, bacteria were grown at $37{ }^{\circ} \mathrm{C}$, in a static incubator if on solid media or at $200 \mathrm{rpm}$ if in liquid media. For short term storage strains were kept at $4{ }^{\circ} \mathrm{C}$ on solid agar plates. For long term storage liquid cultures were mixed with 1:1 with autoclaved $80 \%$ (v/v) glycerol and stored indefinitely at $-80{ }^{\circ} \mathrm{C}$.

\subsection{Standard molecular biology protocols}

\subsubsection{Isolation of genomic DNA (gDNA)}

Bacterial $3 \mathrm{ml}$ overnight cultures were grown in conditions as appropriate for the strain (see Table 2-3) and gDNA was extracted using the DNeasy ${ }^{\mathrm{TM}}$ Blood \& Tissue kit (Qiagen; GmbH, Germany), following the manufacturer's instructions. 


\subsubsection{Isolation of plasmid DNA}

\subsubsection{Miniprep}

Preparation of plasmid DNA was carried out using either the Zyppy ${ }^{\mathrm{TM}}$ Plasmid Miniprep kit (Zymo Research; Irvine, CA, USA) or the High-Speed Plasmid Mini Kit (Geneaid Biotech Ltd; New Taipai City, Taiwan) according to the manufacturer's instructions.

\subsubsection{Midiprep}

DNA midipreps of plasmid F279:ntr were carried out using the Compact Prep ${ }^{\circledR}$ Plasmid Midi Kit (Qiagen, GmbH, Germany) according to manufacturer's instructions. DNA midipreps to provide large quantities of vector for the cloning of mutant gene libraries were prepared using a standard alkaline phosphate midiprep protocol from Sambrook (Sambrook and Russell, 2001).

\subsubsection{Polymerase chain reaction (PCR) protocol}

Where PCR products were to be used in downstream cloning applications, amplification was carried out using Phusion ${ }^{\mathrm{TM}}$ high-fidelity polymerase. For colony screening and diagnostic PCR purposes Biomix Red ${ }^{\mathrm{TM}}$ was used. Standard amplification was carried out as shown in Table 2-6 with PCR components shown in Table 2-7. Protocols for overlap PCR are described in section $\mathbf{2 . 8}$.

\begin{tabular}{|c|c|}
\hline PhusionTM $^{\text {TM }}$ & \\
\hline Temperature & Time \\
\hline $98^{\circ} \mathrm{C}$ & $1 \mathrm{~min}$ \\
\hline $98^{\circ} \mathrm{C}$ & 15 secs \\
\hline $56^{\circ} \mathrm{C}$ & $30 \mathrm{secs} / \mathrm{kB}$ \\
\hline $72{ }^{\circ} \mathrm{C}$ & $30 \mathrm{secs} / \mathrm{kB}$ \\
\hline $72{ }^{\circ} \mathrm{C}$ & $5 \mathrm{mins}$ \\
\hline
\end{tabular}

\begin{tabular}{|c|c|c|}
\hline \multicolumn{2}{|l|}{ Biomix $\operatorname{Red} d^{\mathrm{TM}}$} & \\
\hline Temperature & Time & \\
\hline $95^{\circ} \mathrm{C}$ & 5 mins & \\
\hline $95^{\circ} \mathrm{C}$ & $20 \mathrm{secs}$ & \\
\hline $56^{\circ} \mathrm{C}$ & $30 \operatorname{secs} / \mathrm{kB}$ & 15 cycles \\
\hline $72{ }^{\circ} \mathrm{C}$ & $1 \mathrm{~min} / \mathrm{kB}$ & \\
\hline $95^{\circ} \mathrm{C}$ & $20 \mathrm{secs}$ & \\
\hline $52{ }^{\circ} \mathrm{C}$ & $30 \mathrm{secs}$ & $15-20$ cycle \\
\hline $72{ }^{\circ} \mathrm{C}$ & $1 \mathrm{~min} / \mathrm{kB}$ & \\
\hline $72^{\circ} \mathrm{C}$ & 5 mins & \\
\hline
\end{tabular}

Table 2-6 Standard PCR parameters 
PhusionTM

\begin{tabular}{ll}
\hline Component & $\begin{array}{l}\text { Quantity added } \\
(\mathbf{2 0} \boldsymbol{\mu L} \text { reaction })\end{array}$ \\
\hline $5 \mathrm{X}^{2}$ husion \\
$10 \mathrm{mM}$ HF buffer & $4 \mu \mathrm{L}$ \\
Phusion $^{\mathrm{TM}}$ polymerase & $0.4 \mu \mathrm{L}$ \\
$10 \mu \mathrm{M}$ forward primer & $0.2 \mu \mathrm{L}$ \\
$10 \mu \mathrm{M}$ reverse primer & $1 \mu \mathrm{L}$ \\
Template DNA $(<100 \mathrm{ng} / \mu \mathrm{L})^{\mathrm{a}}$ & $0.5-1 \mu \mathrm{L}$ \\
dd $_{2} \mathrm{O}$ & to $20 \mu \mathrm{L}$ \\
\hline
\end{tabular}

Biomix TM Red

\begin{tabular}{ll}
\hline Component & $\begin{array}{l}\text { Quantity added (20 } \\
\boldsymbol{\mu L} \text { reaction) }\end{array}$ \\
\hline Biomix $^{\mathrm{TM}}$ Red & $10 \mu \mathrm{L}$ \\
$10 \mu \mathrm{M}$ forward primer & $1 \mu \mathrm{L}$ \\
$10 \mu \mathrm{M}$ reverse primer & $1 \mu \mathrm{L}$ \\
Template DNA $(<100 \mathrm{ng} / \mu \mathrm{L})^{\mathrm{a}}$ & $0.5-1 \mu \mathrm{L}$ \\
$\mathrm{ddH}_{2} \mathrm{O}$ & to $20 \mu \mathrm{L}$ \\
\hline
\end{tabular}

${ }^{\mathrm{a}}$ For PCR screening of colonies, small piece of colony template was added to reaction using a sterile pippette tip

Table 2-7 Components of Phusion ${ }^{\mathrm{TM}}$ and Biomix ${ }^{\mathrm{TM}}$ Red PCR reactions

\subsubsection{Agarose gel electrophoresis}

When analysis of DNA samples was necessary, e.g. for examining results of PCR reactions or restriction digests, aliquots of DNA were run on agarose gels $(1 \% \mathrm{w} / \mathrm{v}$ agarose in $1 \mathrm{x}$ TAE buffer (40 mM Tris-acetate, $1 \mathrm{mM}$ EDTA), $1 \mu \mathrm{g} / \mathrm{mL}$ ethidium bromide) covered with 1 x TAE at 120-140 V for 20-40 min. DNA was visualised under UV (ultra violet) light and size determined by comparison to Hyperladder I, (Bioline, London, $U K)$.

\subsubsection{DNA product purification}

DNA products were purified using the DNA Clean and Concentrator ${ }^{\mathrm{TM}}-5$ Kit (Zymo Research; Irvine, CA, USA) according to manufacturer's instructions. The DNA products that required purification included PCR products, DNA following restriction digest for cloning reactions and ligations prior to transformation of electrocompetent $E$. coli.

\subsubsection{Restriction enzyme digest}

When restriction digests were required for standard gene cloning and diagnostic purposes, digests were carried out as per manufacturer's instructions and purified using DNA Clean and Concentrator ${ }^{\mathrm{TM}_{-} 5}$ columns (section 2.5.5). The modified restriction digestion protocol used to generate high insert ratio mutant gene libraries is detailed in $\mathbf{2 . 5 . 8}$. 


\subsubsection{Ligation}

Bioline T4 DNA Ligase was used for ligation reactions as shown in Table 2-8. Ligations were incubated overnight at $16{ }^{\circ} \mathrm{C}$ before being used to transform cells.

\begin{tabular}{ll}
\hline Component & Quantity \\
\hline Vector & $<40 \mathrm{ng}$ \\
Insert & $<160 \mathrm{ng}$ \\
10X buffer & $2 \mu \mathrm{L}$ \\
Ligase $\left(1 \mathrm{U} \cdot \mu \mathrm{L}^{-1}\right)$ & $1 \mu \mathrm{L}$ \\
& To make up \\
$\mathrm{ddH}_{2} \mathrm{O}$ & to $20 \mu \mathrm{L}$ \\
\hline
\end{tabular}

Table 2-8 Ligation components

\subsubsection{Cloning of high insert ratio mutant gene libraries}

All mutant libraries in this thesis were cloned into a pUCX expression vector. For generation of mutant gene libraries, which ideally required $>85 \%$ of the plasmids to carry the mutant gene insert, certain modifications to restriction digest and ligation protocols were made. We found that higher insert ratios could be more routinely achieved if the vector source was digested pUCX:AzoR_Ec plasmid rather than digested pUCX empty (i.e. a "stuffer" region of DNA could be excised to ensure that both restriction sites in the vector had been digested). Prior to digestion the vector $(\sim 10 \mu \mathrm{g})$ was heated to $70{ }^{\circ} \mathrm{C}$ for 10 min before cooling to $37^{\circ} \mathrm{C}$. Vector and insert $(\sim 1 \mu \mathrm{g})$ were incubated with restriction enzymes (10 Units of each enzyme per $1 \mu \mathrm{g}$ DNA) for $16 \mathrm{~h}$ at $37{ }^{\circ} \mathrm{C}$ with DNA concentration kept $<20 \mu \mathrm{g} \cdot \mathrm{mL}^{-1}$. After $16 \mathrm{~h}$ an additional 10 Units of each restriction enzyme, and an additional enzyme BamHI-HF, (which cuts once in the AzoR_Ec gene sequence but not in the pUCX vector backbone) was added to each digest. The digests were incubated for another hour and then the restriction enzymes heat inactivated according to the manufacturer's instructions. Digested insert was cleaned up on a DNA Clean and Concentrator ${ }^{\mathrm{TM}_{-} 5}$ column (Section 2.5.5) and digested vector DNA was cleaned up using three individual Miniprep columns and eluted in pre warmed MQ. DNA was stored in single use aliquots at $-20^{\circ} \mathrm{C}$ or used immediately in ligation protocols.

\subsubsection{DNA quantification}

Where required, purity and concentration of DNA products were determined using a NanoDrop ND-1000 spectrophotometer (Thermo Fisher Scientific; Waltham, MA, USA), according to the manufacturer's instructions. 


\subsubsection{DNA sequencing}

Sequencing was carried out by Macrogen Inc. (Seoul, South Korea) to determine the sequence of any mutants of interest and verify correct insertion and sequence of every NTR gene post insertion into a novel vector backbone.

\subsubsection{Transformation/electroporation of $E$. coli}

\subsubsection{Generation of chemically competent cells}

An overnight culture of the strain to be made competent was grown in $3 \mathrm{ml} \mathrm{LB}$ amended with $10 \mathrm{mM} \mathrm{MgCl}$ and any relevant antibiotic. The overnight culture was inoculated into a 10-50 mL culture of $\mathrm{LB}+10 \mathrm{mM} \mathrm{MgCl}_{2}$ at a 50-fold dilution and the culture was grown until an $\mathrm{OD}_{600}$ of 0.4-0.6 was reached. When this was achieved the culture was placed on ice for $15 \mathrm{~min}$, spun down (4000 $\mathrm{rpm} 4{ }^{\circ} \mathrm{C}, 15 \mathrm{~min}$ ), the supernatant removed and the cell pellet resuspended in a $0.8 \mathrm{x}$ volume of ice cold TFBI. The culture was then left on ice for $2 \mathrm{~h}$, spun down (4000 rpm $4{ }^{\circ} \mathrm{C}, 15 \mathrm{~min}$ ), the TFBI discarded and the supernatant resuspended in $0.1 \times$ volume of ice cold TFBII. Aliquots $(50-200 \mu \mathrm{L})$ of the cells were then transferred into ice cold $1.5 \mathrm{ml}$ microcentrifuge tubes and either immediately transformed with plasmid DNA or snap frozen and stored indefinitely at $-80{ }^{\circ} \mathrm{C}$.

\section{TFBI}

Potassium acetate $30 \mathrm{mM}$

$\mathrm{MnCl}_{2} 50 \mathrm{mM}$

$\mathrm{CaCl}_{2} 10 \mathrm{mM}$

Glycerol 15\% (w/v)

\section{TFBII}

MOPS, pH $7.010 \mathrm{mM}$

$\mathrm{CaCl}_{2} 75 \mathrm{mM}$

$\mathrm{KCl} 10 \mathrm{mM}$

Glycerol 15\% (w/v)

\subsubsection{Transformation of chemically competent cells}

Frozen aliquots of cells to be transformed were removed from $-80{ }^{\circ} \mathrm{C}$ storage and fully defrosted on ice. DNA to be transformed into the cells was added and the mix left on ice for $15 \mathrm{~min}$. Ligations to be transformed were added to the cells at $<10 \%$ of the total cell volume. The cells were then heat shocked by incubation in a $42{ }^{\circ} \mathrm{C}$ heat block for $90 \mathrm{~s}$ and placed back on ice for 3-5 min. $900 \mu \mathrm{L}$ of LB was added to the cell mix and the cells allowed to recover at $37{ }^{\circ} \mathrm{C}, 200 \mathrm{rpm}$ for 45-60 min. Cells were then pelleted (13,000 $\mathrm{rpm}, 30 \mathrm{~s}$ ), the supernatant removed, cells resuspended in $200 \mu \mathrm{L}$ of LB and spread onto 
an agar plate containing the appropriate antibiotics. Plates were incubated overnight at 37 ${ }^{\circ} \mathrm{C}$.

\subsubsection{Generation of electrocompetent cells}

A $10 \mathrm{~mL}$ overnight culture of the strain to be made competent was inoculated from verified glycerol stocks. This overnight was used to inoculate a $500 \mathrm{~mL}$ flask containing LB and appropriate antibiotics and the resulting culture was grown at $37{ }^{\circ} \mathrm{C}, 200 \mathrm{rpm}$ until an $\mathrm{OD}_{600}$ of $0.35-0.4$ was reached, at which point the culture was transferred into 10 sterile $50 \mathrm{ml}$ tubes and placed on ice for 15-30 min with occasional swirling. The cells were then collected by centrifugation $\left(2500 \mathrm{rpm}, 30 \mathrm{~min}, 4{ }^{\circ} \mathrm{C}\right)$ supernatant discarded and the pellets resuspended in a total volume of $500 \mathrm{~mL}$ ice cold sterile $\mathrm{ddH}_{2} \mathrm{O}$ by gentle pipetting. Following this first washing step the cells were again collected by centrifugation $\left(2500 \mathrm{rpm}, 30 \mathrm{~min}, 4{ }^{\circ} \mathrm{C}\right.$ ) and the resulting pellet resuspended in $250 \mathrm{ml}$ ice-cold sterile $10 \% \mathrm{v} / \mathrm{v}$ glycerol. The cells were again pelleted by centrifugation (2500 $\mathrm{rpm}, 30 \mathrm{~min}, 4{ }^{\circ} \mathrm{C}$ ) and the resulting pellet resuspended in $125 \mathrm{ml}$ ice cold sterile $10 \% \mathrm{v} / \mathrm{v}$ glycerol. The cells were again collected by centrifugation and the pellet resuspended in $500 \mu \mathrm{L}$ ice cold sterile GYT. The $\mathrm{OD}_{600}$ of a 1/100 dilution of the cell mixture was then determined and GYT added to give a final concentration of $2-3 \times 10^{10}$ cells $/ \mathrm{mL}\left(\mathrm{OD}_{600}\right.$ $1.0=2.5 \times 10^{8}$ cells $\left./ \mathrm{ml}\right) .40 \mu \mathrm{L}$ aliquots were then distributed into pre-chilled $1.5 \mathrm{~mL}$ microcentrifuge tubes on ice. Cell aliquots were then snap-frozen using a metal tube block cooled to $-80{ }^{\circ} \mathrm{C}$. Frozen aliquots of cells were stored at $-80{ }^{\circ} \mathrm{C}$ until needed.

\subsubsection{Electroporation of electrocompetent cells}

Aliquots of frozen cells $(40 \mu \mathrm{L})$ were defrosted on ice. DNA to be electroporated was first purified and eluted in $\mathrm{ddH}_{2} \mathrm{O}$. Then 25-50 ng of DNA added to the cells, mixed, and the cells left on ice for a further $15 \mathrm{~min}$. The cells were then moved to ice cold $0.2 \mathrm{~mm}$ gap electroporation cuvettes and electroporated using a Bio-Rad GenePulser Xcell ${ }^{\mathrm{TM}}$ (Hercules, CA, USA). The cells were pulsed $(2500 \mathrm{~V}, 25 \mu \mathrm{F}, 200 \Omega)$ and $1 \mathrm{~mL}$ of room temperature SOC added immediately after. Cells were allowed to recover for $60 \mathrm{~min}$ at 37 ${ }^{\circ} \mathrm{C}, 200 \mathrm{rpm}$ before plating on the appropriate antibiotic agar plate to select for positive transformants. Modifications to this protocol when used for the generation of large mutant libraries which were to be assessed by FACS are presented in section $\mathbf{2 . 1 5}$. 


\subsection{Gene knockout in $E$. coli}

Genes were knocked out using the FLP/FRT recombinase system developed by Datsenko and Wanner (2000). Firstly a primer pair were designed that contained, at the 5' ends, 30 nt extensions homologous to the $30 \mathrm{bp}$ at beginning and end of the gene to be removed, with the 3 ' ends of the primers able to act as a priming sequence and allow amplification of a $\sim 1500 \mathrm{~kb}$ section of the plasmid pKD4. This PCR amplified pKD4 sequence contained a kanamycin resistant $\left(\mathrm{Kan}^{\mathrm{R}}\right)$ cassette, flanked by flippase recognition target (FRT) excision sites. Through homologous recombination at the $30 \mathrm{nt}$ regions of homology, the targeted gene is replaced with the exogenous $\operatorname{Kan}^{\mathrm{R}}$ cassette. To allow this homologous recombination to occur, the strain in which the gene is to be removed is first transformed with the bacteriophage plasmid pKD46, which when expressed by E. coli allows for the homologous recombination of short linear DNA. Upon L-arabinose dependent induction of pKD46, followed by electroporation, the pKD4 fragment is inserted into the genomic DNA, knocking out the gene of interest. To remove the $\mathrm{Kan}^{\mathrm{R}}$ cassette cultures were grown at $42{ }^{\circ} \mathrm{C}$ to allow for removal of heat sensitive pKD46 followed by transformation of the strain with the plasmid pCP20. pCP20 contains an ampicillin resistant cassette and encodes for a FLP recombinase which, on temperature dependent induction, recognizes the FRT excision sequence and removes the $\operatorname{Kan}^{\mathrm{R}}$ cassette. Further high temperature culturing eventually cures the strain of the heat sensitive pCP20 leaving only a 200 bp "scar" in place of the knocked out gene.

\subsection{NTR candidate library}

The 58 bacterial oxidoreductase enzymes tested for activity with various nitroaromatic substrates are shown in Table 2-9. Each was individually PCR amplified from gDNA preparations of indicated bacterial species and cloned using restriction digest into the pUCX expression vector before transformation into specialised E. coli assay strains. 


\begin{tabular}{|c|c|c|c|c|}
\hline Gene & Organism NTR originated from & Family & $\%$ ID/Sim ${ }^{\mathrm{a}}$ & Accession number \\
\hline NfsA_Ec & Escherichia coli & NfsA & $100 / 100$ & AP_001482 \\
\hline NfsA_St & Salmonella enterica & NfsA & $87 / 95$ & NP_455401 \\
\hline NfsA_Ck & Citrobacter koseri & NfsA & $86 / 92$ & YP_001453815* \\
\hline NfsA_Kp & Klebsiella pneumoniae & NfsA & $83 / 92$ & YP_001334548 \\
\hline NfsA_Es & Enterobacter sakazakii & NfsA & $82 / 94$ & YP_001438568 \\
\hline NfsA_Eca ${ }^{b}$ & Erwinia carotovara & NfsA & $65 / 82$ & YP_050771* \\
\hline NfsA_Vf & Vibrio fischeri & NfsA & $52 / 68$ & YP_002158635* \\
\hline NfsA_Vv & Vibrio vulnificus & $\mathrm{NfsA}$ & $51 / 65$ & NP_763284* \\
\hline Frp_Vh & Vibrio harveyi $^{c}$ & NfsA & $51 / 65$ & AAM73721* \\
\hline CO-Frp_Vh & Vibrio harveyi $^{c}$ & $\mathrm{NfsA}$ & $51 / 65$ & AAA21331 \\
\hline NfrA_Bs & Bacillus subtilis & NfsA & $39 / 62$ & CAB15837* \\
\hline NfsA_Lw & Listeria welshimeri & NfsA & $41 / 60$ & YP_849114 \\
\hline NfsA_Li & Listeria innocua & NfsA & $39 / 60$ & NP_470273 \\
\hline NfsA_Bc & Bacillus coagulans & NfsA & $38 / 60$ & ZP_01697274* \\
\hline NfsA_Np & Nostoc punctiforme & $\mathrm{NfsA}$ & $38 / 59$ & ZP_00110199 \\
\hline NfsA_Bt & Bacillus thuringiensis & NfsA & $35 / 58$ & YP_035832* \\
\hline NfsA_Ls & Lactobacillus sakei & NfsA & $40 / 57$ & YP_396325* \\
\hline EcD_Pp & Pseudomonas putida & NfsA & $35 / 57$ & AAL09699 \\
\hline YcnD_Bs & Bacillus subtilis & NfsA & $35 / 56$ & CAB12194* \\
\hline NfsA_Ms & Mycobacterium smegmatis & $\mathrm{NfsA}$ & $36 / 54$ & YP_887240 \\
\hline NfsB_Ec & Escherichia coli & $\mathrm{NfsB}$ & $100 / 100$ & AP_001223 \\
\hline NfsB_Ck & Citrobacter koseri & $\mathrm{NfsB}$ & $88 / 95$ & YP_001454141* \\
\hline NfsB_St & Salmonella enterica & $\mathrm{NfsB}$ & $88 / 91$ & NP_806031. \\
\hline NfsB_Kp & Klebsiella pneumoniae & $\mathrm{NfsB}$ & $84 / 93$ & YP_001334233* \\
\hline NfsB_Vv & Vibrio vulnificus & $\mathrm{NfsB}$ & $61 / 75$ & NP_762888* \\
\hline NfsB_Pp & Pseudomonas putida & $\mathrm{NfsB}$ & $52 / 71$ & NP_744580 \\
\hline NfsB_Es & Enterobacter sakazakii & $\mathrm{NfsB}$ & $46 / 66$ & YP_001437964* \\
\hline FraseI_Vf & Vibrio fischeri & $\mathrm{NfsB}$ & $34 / 53$ & BAA04595 \\
\hline NfsB_Vh & Vibrio harveyi & $\mathrm{NfsB}$ & $30 / 48$ & ZP_01985918* \\
\hline YfkO_Bs & Bacillus subtilis & $\mathrm{NfsB}$ & $29 / 46$ & CAB12612* \\
\hline YdgI_Bs & Bacillus subtilis & $\mathrm{NfsB}$ & $25 / 47$ & CAB12385* \\
\hline $\mathrm{NfsB} B \mathrm{~Pa}$ & Pseudomonas aeruginosa & $\mathrm{NfsB}$ & $24 / 42$ & NP_253877 \\
\hline AzoR_Ec & Escherichia coli & AzoR & $100 / 100$ & AP_002037 \\
\hline AzoR_St & Salmonella enterica & AzoR & $87 / 95$ & NP_805329. \\
\hline AzoR_Vv & Vibrio vulnificus & AzoR & $56 / 73$ & NP_761189* \\
\hline AzoR_Pp & Pseudomonas putida & AzoR & $48 / 66$ & NP_746647 \\
\hline NemA_Ec & Escherichia coli & NemA & $100 / 100$ & AP_002272 \\
\hline NemA_St & Salmonella enterica & NemA & $92 / 96$ & NP_805105* \\
\hline NemA_Kp & Klebsiella pneumoniae & NemA & $86 / 92$ & YP_001335650* \\
\hline NemA_Vv & Vibrio vulnificus & NemA & $47 / 65$ & NP_762994* \\
\hline
\end{tabular}




\begin{tabular}{|l|l|l|l|l|}
\hline YwrO_Bs & Bacillus subtilis & YwrO & $70 / 82^{\mathrm{d}}$ & NP_391480* \\
\hline YwrO_Li & Listeria innocua & YwrO & $42 / 57^{\mathrm{d}}$ & NP_471232* \\
\hline YwrO_Vf & Vibrio fischeri & YwrO & $47 / 62^{\mathrm{d}}$ & YP_002157853 \\
\hline YieF_Ec & Escherichia coli & YieF & $100 / 100$ & AP_004074 \\
\hline YieF_Pa & Pseudomonas aeruginosa & YieF & $45 / 60$ & NP_249895 \\
\hline MdaB_Ec & Escherichia coli & MdaB & $100 / 100$ & AP_003578 \\
\hline MdaB_Ps & Pseudomonas syringae & MdaB & $60 / 74$ & YP_273002 \\
\hline WrbA_Ec & Escherichia coli & WrbA & $100 / 100$ & AP_001635 \\
\hline WrbA_Ps & Pseudomonas syringae & WrbA & $39 / 55$ & YP_275866 \\
\hline YdjA_Ec & Escherichia coli & YdjA & $100 / 100$ & AP_002384 \\
\hline YdjA_Kp & Klebsiella pneumoniae & YdjA & $79 / 89$ & YP_001334868 \\
\hline NQO1_Pp & Pseudomonas putida & NQO1 & $37 / 56^{\mathrm{e}}$ & NP_745853 \\
\hline NQO1_Pa & Pseudomonas aeruginosa & NQO1 & $41 / 54^{\mathrm{e}}$ & NP_253662 \\
\hline YcdI_Ec & Escherichia coli & YcdI & $100 / 100$ & AP_001639 \\
\hline YcdI_Kp & Klebsiella pneumoniae & YcdI & $76 / 85$ & YP_001334699 \\
\hline YcaK_Ec & Escherichia coli & YcaK & $100 / 100$ & AP_001531 \\
\hline YcaK_Pa & Pseudomonas aeruginosa & YcaK & $27 / 48$ & NP_249916 \\
\hline KefF_Ec & Escherichia coli & KefF & $100 / 100$ & AP_000710 \\
\hline
\end{tabular}

${ }^{a}$ ID and \% Sim. indicate amino acid identity and similarity, (as calculated by the blastp program "identities" and "positives", respectively) of each NTR to the E. coli orthologue within enzyme family unless otherwise stated

${ }^{\mathrm{b}}$ Three letter species abbreviation to differentiate from Nfs A_Ec

${ }^{c}$ Frp and CO-Frp from Vibrio harveyi strains KCTC 2720 and ATCC 33843 respectively

${ }^{\mathrm{d}} \mathrm{Sim}$. and ID values for all YwrO family enzymes are relative to B. amyloliquefaciens YwrO

${ }^{\mathrm{e}} \mathrm{Sim}$. and ID values are relative to Rattus norvegicus NQO1/DTD

*Due to differing strain isolate exact NTR protein sequence slightly differs from Accession number and can be found in Appendix 1

Table 2-9 58 oxidoreductase enzymes in the expanded NTR candidate library, cloned into E. coli expression vector pUCX. Where applicable previously published enzyme names are used, otherwise names indicate the enzyme family followed by a two letter abbreviation from the genus and species of bacteria the enzyme originated from. Strains used as NTR sources are as indicated in Table 2-3.

\subsubsection{Bioinformatics}

Protein homology searches were carried out using the National Centre for Biotechnology Information (NCBI) protein database using the algorithm protein-protein BLAST (blastp; http://blast.ncbi.nlm.nih.gov/)

\subsection{Overlap PCR}

Mutant NTR genes were created using overlap PCR. For each mutation to be made, two internal gene primers were designed. The "Fwd" primer was comprised of three parts, first a $\sim 18 \mathrm{nt}$ section homologous to the gene section immediately upstream of the 
mutation site, next the actual mutant codon and finally $\sim 15 \mathrm{nt}$ homologous to the gene region immediately after the mutation site. The "Rev" primer was the reverse complement of the first $\sim 18$ nucleotides of the "Fwd" primer. An example of such primers can be seen in Figure 2-3.

\author{
NfsB EC T41L Fwd CAATACAGCCCATCCAGCCTGAACTCCCAGCCGTGG \\ NfsB EC 41 Rev GCTGGATGGGCTGTATTG
}

Partial NfsB (E.c) sequence

(bases 95-145)

-CGCTACTGCAATACAGCCCATCCAGCACCAACTCCCAGCCGTGGCATTTATTG-

Figure 2-3 Example of primers used to generate site directed mutants. Primers designed to generate NfsB_Ec T41L mutant from NfsB_Ec wild type. Site of mutation marked in red and primer overlap region in blue. Displayed 5'-3' direction.

A schematic of how such internal primers, in combination with gene specific primers, can be used to generate a full length gene using overlap PCR can be seen in Figure 2-4.

1)
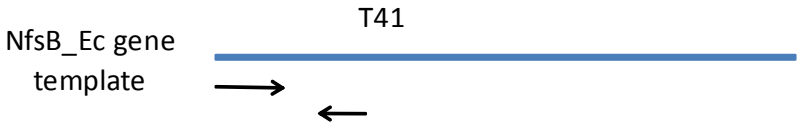

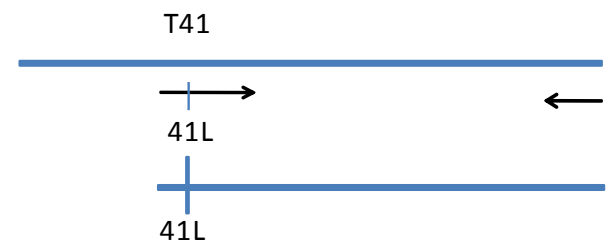

2)

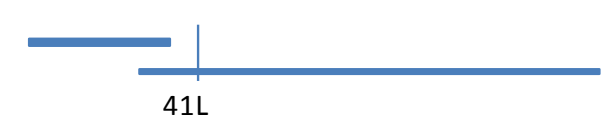

3)

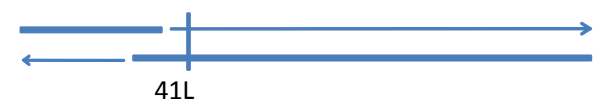

4)

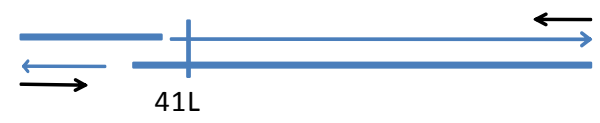

5)

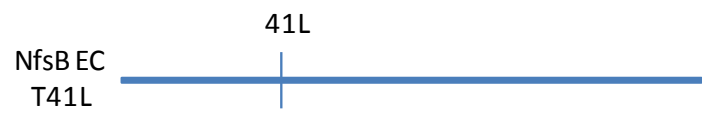

Figure 2-4 Schematic of overlap PCR for generation of site directed mutants 1) Two gene fragments are separately amplified using the wild type gene as template with one internal primer and complementary gene specific primer in each reaction. The second fragment contains the mutation specified by the internal primer. 2) These fragments, which have a $\sim 18$ base pair overlap due to the specific design of the internal primers are combined. 3) A primer-less PCR reaction is commenced and due to this overlap region the fragments self- prime. 4) After a number of PCR cycles gene specific primers are added and increase amplification of the full length gene, which now contains the specified mutation. 
The initial gene fragments were amplified in separate PCR reactions from a pUCX: wild type NTR gene template ( 500 pg of plasmid per reaction), using a standard Phusion ${ }^{\mathrm{TM}}$ protocol (2.5.3). PCR products were then purified, combined in a 1:1 molar ratio and a full length gene product amplified using an overlap PCR protocol Figure 2-5. When multiple mutations at different gene sites were simultaneously introduced, multiple fragments of the gene were amplified and then combined in an overlap PCR reaction, with the initial amplification of internal fragments carried out using complementary internal gene primers.

\begin{tabular}{|c|c|c|}
\hline \multicolumn{2}{|l|}{ Phusion $^{\mathrm{TM}}$} & \\
\hline Temperature & Time & \\
\hline $98^{\circ} \mathrm{C}$ & $2 \mathrm{~min}$ & \\
\hline $98^{\circ} \mathrm{C}$ & $10 \mathrm{secs}$ & \\
\hline $46-52^{\circ} \mathrm{C}$ & $20 \mathrm{sec}$ & 15 cycles \\
\hline $72{ }^{\circ} \mathrm{C}$ & $30 \mathrm{secs} / \mathrm{kB}$ & \\
\hline Pause & Add gene & ecific prime $r$ \\
\hline $98^{\circ} \mathrm{C}$ & $10 \mathrm{secs}$ & \\
\hline $56^{\circ} \mathrm{C}$ & 20 secs & $15-20$ cycles \\
\hline $72{ }^{\circ} \mathrm{C}$ & $30 \mathrm{secs} / \mathrm{kB}$ & \\
\hline $72{ }^{\circ} \mathrm{C}$ & 5 mins & \\
\hline
\end{tabular}

Figure 2-5 Overlap PCR protocol Both initial fragment annealing temperature and the number of replication cycles post primer addition were occasionally varied to increase yield of a specific full length product.

\subsection{Generation of mutant NTR libraries}

In addition to being used to introduce specific mutations, overlap PCR was also used to construct mutant gene libraries. Here the mutant codon(s) introduced contained redundant bases to allow the site to code for any one of multiple amino acids. An NNK codon was used in site saturation mutagenesis libraries, and restricted redundancy codons used when constructing mutant libraries designed to encompass all possible combinations of a small number of preselected mutations. In one instance a mutant gene library was ordered and synthesised by GenScript (GenScript, NJ, USA). Post delivery of this library it was digested and cloned into our pUCX expression vector as per the gene libraries constructed in house. 
Once the full length mutant gene was constructed for a library it was cloned into the pUCX over-expression vector, and the ligations either transformed into chemically competent E. coli SOS-R2 cells or electrocompetent E. coli 7KO-pGFP cells as appropriate. Entire transformations (SOS-R2 cells) or a $20 \mu \mathrm{L}$ aliquot (7KO-pGFP) were spread onto solid media + antibiotic plates for selection of transformed colonies, and 1015 random colonies were PCR screened with NfsA insert specific primers to determine the gene insert ratio of each library. For SOS-R2 libraries this insert ratio, in conjunction with the calculation of the number of possible variants existing in each library, was used to determine how many individual colonies would have to be screened to ensure a $>95 \%$ probability that every variant had been tested at least once (Reetz and Carballeira, 2007). Libraries with colony numbers not less than this were then picked individually into separate media filled wells of 96 well microplates. These plates were grown overnight, mixed with glycerol and then stored at $-80{ }^{\circ} \mathrm{C}$. Glycerol plates were used to inoculate day cultures for screening of mutants activities with nitroaromatic substrates. Treatment of 7KO-pGFP libraries which were to be assessed by FACS is further described in section 2.9.1)

\subsubsection{Modifications to electroporation protocol for the generation of mutant libraries which were to be assessed by FACS}

Here the initial electroporation protocol was identical to that of section 2.5.11, but positive transformants were selected by adding cells post the $60 \mathrm{~min}$ recovery period to $50 \mathrm{~mL}$ LB, Amp + Spec liquid media and the flasks left overnight with gentle agitation at room temperature. The overnight cultures were then spun down and the cell pellet resuspended in $1 \mathrm{~mL} \mathrm{LB}+\mathrm{Amp}$ media. The $\mathrm{OD}_{600}$ absorbance of a diluted aliquot was read to allow an estimation of the cell number of the library and the remainder mixed in a 50:50 (v/v) ratio with $80 \%$ glycerol and stored in $200 \mu \mathrm{L}$ aliquots at $-80{ }^{\circ} \mathrm{C}$ until required for assessment of mutant activity using FACS.

\subsection{Protein purification}

\subsubsection{Expression using Autoinduction media}

A single colony or small inoculum of $E$. coli BL21(DE3) carrying pET28a(+):ntr was used to inoculate $50 \mathrm{~mL}$ ZYP-5052 + Kan. This culture was left to incubate overnight at $30{ }^{\circ} \mathrm{C}, 200 \mathrm{rpm}$ for at least $16 \mathrm{~h}$, before chilling on ice for $15 \mathrm{~min}$. The cell pellet was then collected by centrifugation $\left(4,000 \mathrm{rpm}, 25 \mathrm{~min}, 4{ }^{\circ} \mathrm{C}\right)$. After discarding the 
supernatant, the cell pellet was either processed immediately, or stored at $-80{ }^{\circ} \mathrm{C}$ until required.

\subsubsection{Expression using low temperature LB + supplements}

A $1.5 \mathrm{~mL}$ volume of overnight culture of $E$. coli BL21(DE3) carrying pET28a(+):ntr was used to inoculate a $50 \mathrm{~mL}$ volume of $\mathrm{LB}+\mathrm{Kan}+2.5 \mathrm{mM}$ Betaine $+1 \mathrm{M}$ D-sorbitol media. This culture was grown at $37{ }^{\circ} \mathrm{C}, 200 \mathrm{rpm}$ until an $\mathrm{OD}_{600}$ of $\sim 0.5$ was reached. Following this the temperature was lowered to $18{ }^{\circ} \mathrm{C}$ and after $45 \min 0.5 \mathrm{mM}$ IPTG added to induce NTR over-expression. Cultures were left to grow for $\sim 40 \mathrm{~h}$ before the cell pellet was collected as per 2.10.1.

\subsubsection{Enzyme purification}

Cell pellets were lysed by the addition of $4 \mathrm{~mL}$ Bugbuster® (Novagen; Merck Millipore, $M A, U S A$ ) for every $50 \mathrm{~mL}$ of initial culture. The pellets were resuspended and incubated at room temperature on an orbital shaker for 20 min prior to removal of the insoluble fraction by centrifugation for $15 \mathrm{~min}$ at $4{ }^{\circ} \mathrm{C}, 12,000 \mathrm{rpm}$. Purification of $\mathrm{His}_{6}$ - tagged proteins from the soluble fraction was carried out using a Novagen Hisbind ${ }^{\mathrm{TM}}$ Ni-NTA chromatography kit (Merck Millipore, MA, USA), according to the manufacturer's instructions. The final settled-bed resin volume per column was $\sim 1 \mathrm{~mL}$. Proteins were eluted in $500 \mu \mathrm{L}$ fractions and the three most yellow fractions by eye were pooled and incubated with excess $(1 \mathrm{mM})$ FMN or FAD cofactor as appropriate for $1 \mathrm{~h}$ on ice. Buffer-exchange into $40 \mathrm{mM}$ Tris- $\mathrm{Cl}$ pH 7.0 was then conducted using a $5 \mathrm{~mL}$ HiTrap $^{\mathrm{TM}}$ Desalting column (GE Healthcare, Chalfont St. Giles, UK) according to the manufacturer's instructions. Only $1 \mathrm{~mL}$ of column flow-through was collected upon elution to minimize potential contamination with excess free FMN.

In one instance $\mathrm{His}_{6}$-tagged NfsA_St was prepared by passage through a French press. Here cell pellets were resuspended in ice cold $10-20 \mathrm{~mL} 1 \times$ His $^{\bullet}$ Bind $^{\mathrm{TM}}$ Binding buffer (Novagen Hisbind $^{\mathrm{TM}}$ Ni-NTA chromatography kit ) and pressure at 40,000 psi applied three times to achieve uniform cell lysis.

His $_{6}$-tags were not removed from purified proteins as tag presence had previously been determined to not affect the catalytic activity of a wide range of purified NTRs (Prosser, 2011) . 


\subsubsection{Determining enzyme concentration}

Concentrations of purified protein samples were measured using the DCTM protein assay kit (Bio-Rad, Hercules, CA, USA), following the manufacturer's instructions. Protein standards used consisted of Bovine Serum Albumin (BSA) made up in $40 \%$ glycerol, 40 $\mathrm{mM}$ Tris-Cl pH 7.0 and spanning from 0.125-1.5 mg.ml ${ }^{-1}$.

\subsubsection{Enzyme storage}

Immediately post purification samples were mixed 1:1 with $80 \%$ glycerol and stored at $20{ }^{\circ} \mathrm{C}$ until use, typically within a few weeks of purification. Glycerol was found to have no detrimental effects on enzyme activity and when tested NTRs have been found to retain initial activity $>6$ month post storage (results not shown).

\subsection{In vitro purified protein kinetics}

Apparent steady state enzyme kinetics for NTRs and substrates were assessed via monitoring decrease in absorbance at $340 \mathrm{~nm}$, with extinction coefficients determined as outlined in section 1.11.1. Reactions were carried out in $60 \mu \mathrm{L}$ volumes in UVettes ${ }^{\mathrm{TM}}$ (Eppendorf, Hamburg, Germany), using the $2 \mathrm{~mm}$ path length, and the wavelength was measured using a Helios y UV-Vis spectrophotometer (Thermo Fisher Scientific; Waltham, MA, USA). Reactions were in $10 \mathrm{mM}$ Tris-Cl $\mathrm{pH} 7.0$ buffer and consisted of $200 \mu \mathrm{M}$ NAD(P)H, 2\% DMSO and substrate concentrations ranging from 0-1200 $\mu \mathrm{M}$. Reactions were initiated by addition of $6 \mu \mathrm{L}$ of an appropriate dilution of enzyme and the linear decrease in absorbance monitored for the first 20-40 s post enzyme addition. Where possible reaction rates were measured at substrate concentrations ranging from $0.2-5 \mathrm{x}$ apparent $K_{m}$, although for some enzymes this was not possible as the sensitivity and linear absorbance maxima of the spectrophotometer meant only concentrations ranging from $6.25-1200 \mu \mathrm{M}$ substrate could be accurately assayed. Non-linear regression analyses and Michaelis-Menten curve fitting was performed using SigmaPlot ${ }^{\mathrm{TM}} 10.0$ (Systat $^{2}$ Software, Richmond, CA, USA).

\subsubsection{Determination of 2-NI extinction coefficients}

Both the oxidation of $\mathrm{NAD}(\mathrm{P}) \mathrm{H}$ and the reduction of the 2-NI substrates (absorbance maximas typically near $320 \mathrm{~nm}$ (Grunbaum et al., 1987; Olekhnovich et al., 2009)) contributes to the decrease in light absorbance at $340 \mathrm{~nm}$ as the oxidoreductase reaction proceeds (Varghese and Whitmore, 1980). As such, combined molar extinction coefficients encompassing the change in absorbance due to both compounds were derived 
as previously described (Olekhnovich et al., 2009). These values combined the $340 \mathrm{~nm}$ extinction coefficient of the 2-NI compound (experimentally determined) with $\varepsilon=12,440$ $\mathrm{M}^{-1} \mathrm{~cm}^{-1}$ representing the assumed oxidation of two moles of NAD $(\mathrm{P}) \mathrm{H}\left(2 \times \varepsilon=6,220 \mathrm{M}^{-1}\right.$ $\mathrm{cm}^{-1}$ ) for every mole of 2-NI reduced. The extinction coefficient for each nitroimidazole substrate was experimentally determined by measuring the decrease in $340 \mathrm{~nm}$ absorbance that occurred when $100 \mu \mathrm{M}$ of 2-NI was incubated with $300 \mu \mathrm{M}$ NADPH and excess NfsA (purified enzyme) for $30 \mathrm{~min}$. NfsA was used as it possesses low-level NADPH oxidase activity, such that it slowly reduces dissolved oxygen in the buffer, ensuring the eventual oxidation of all NADPH remaining in the cuvette post full reduction of the 2-NI substrate. A list of calculated extinction coefficients is presented in Table 2-10.

\begin{tabular}{ccc}
\hline Compound & $\begin{array}{c}\Delta \mathbf{3 4 0} \mathbf{~ n m}\left(\mathbf{c m}^{-1}\right) \\
\text { when } \mathbf{1 0 0} \boldsymbol{\mu M} \\
\text { reduced }\end{array}$ & $\begin{array}{c}\text { Extinction } \\
\text { coefficient } \\
\left(\mathbf{M}^{-1} \mathbf{c m}^{-\mathbf{1}}\right)\end{array}$ \\
\hline EF5 & -0.651 & 19,000 \\
EF3 & -0.665 & 19,100 \\
F-miso & -0.65 & 18,900 \\
Pimo & -0.611 & 18,600 \\
RB6145 & -0.706 & 19,500 \\
HX4 & -0.666 & 19,000 \\
\hline
\end{tabular}

Table 2-10 Experimentally derived extinction coefficients for 2-NI compounds used in this study, determined at $340 \mathrm{~nm}$ to take into account loss in absorbance that occurs due to both 2-NI reduction and $\mathrm{NAD}(\mathrm{P}) \mathrm{H}$ oxidation.

\subsection{Thermal scanning experiments to determine protein melting temperatures}

Thermal scanning experiments were performed using a Rotor-Gene 6000 (Corbett Research, Concorde, Australia). Experiments were performed as previously published ((Niesen et al., 2007) and http://thermofluor.org/index.html). In brief, duplicate samples containing $5 \mu \mathrm{g}$ protein and Sypro Orange dye (Sigma-Aldrich, St. Louis, MO,USA) in Tris- $\mathrm{HCl}$ pH 7 buffer were subjected to a thermal melt cycle $\left(35-95{ }^{\circ} \mathrm{C}\right)$, using a $5 \mathrm{~s}$ ramp. Temperature induced protein melt was determined by monitoring fluorescence intensity. Data was analysed using Rotor-Gene 6000 Series software 1.7 (Corbett Research, Concorde, Australia). 


\subsection{Sodium Dodecyl Sulphate Polyacrylamide Gel Electrophoresis (SDS-PAGE)}

\subsubsection{SDS-PAGE protocol}

For visualisation of proteins, 15\% SDS-PAGE gels were cast using a Bio-Rad Protean $\mathrm{II}^{\mathrm{TM}}$ apparatus. Roughly $5 \mathrm{~mL}$ of $15 \%$ separating gel (see 2.13.2) was applied to the gel cast, covered with $1 \mathrm{~mL}$ of drum grade $100 \%$ isopropanol and left to set for $30 \mathrm{~min}$. The isopropanol was then discarded and roughly $1.5 \mathrm{~mL}$ of $4 \%$ stacking gel poured over and a multi-well comb inserted and left to solidify for $30 \mathrm{~min}$.

Samples to be run on each SDS-PAGE gel were added in a 2:1 ratio to 3 x SDS-loading buffer. Samples were boiled at $95{ }^{\circ} \mathrm{C}$ for $5 \mathrm{~min}$ prior to electrophoresis. Gels were run in $1 \mathrm{x}$ SDS-Run buffer, at constant voltage $(150 \mathrm{~V})$ for $\sim 60 \mathrm{~min}$, roughly corresponding to the time at which the SDS load buffer ran off the gel. Protein bands were stained by gentle shaking in Coomassie blue stain solution for $30 \mathrm{~min}$. Destaining was carried out by washing the gel of all Coomassie blue stain in running tap water, followed by gentle shaking in destain solution for as long as necessary to achieve the desired contrast. Protein size was determined by comparison to a pre-stained Protein Ladder (10-250 kDa) (NEB; Ipswich, MA, USA) 


\subsubsection{SDS-PAGE recipes}

SDS-PAGE Recipes

$15 \%$ Se perating gel $(10 \mathrm{~mL})$

$2.5 \mathrm{~mL} 1.5 \mathrm{M}$ Tris-Cl (pH 8.8)

$100 \mu \mathrm{L}$ SDS

$2 \mathrm{~mL}$ Bis-Acrylamide

$3.65 \mathrm{~mL}$ Acrylamide

$1.75 \mathrm{~mL} \mathrm{ddH}_{2} \mathrm{O}$

$100 \mu \mathrm{L} 10 \%$ APS $\mid$ Added immediately

$6 \mu \mathrm{L}$ TEMED $\quad$ prior to pouring gel

4\% Stacking gel (3 mL)

$750 \mu \mathrm{L} 0.5 \mathrm{M}$ Tris-Cl (pH 6.8)

$30 \mu \mathrm{L} 10 \%$ SDS

$156 \mu \mathrm{L} \mathrm{2 \%}$ Bis-Acrylamide

$288 \mu \mathrm{L}$ Acryalmide

$1.77 \mathrm{~mL} \mathrm{ddH_{2 } \mathrm { O }}$

$30 \mu \mathrm{L} 10 \%$ APS $\quad$ Added immediately

$5 \mu \mathrm{L}$ TEMED $\quad$ prior to pouring gel

3 x SDS Loading Buffer

$150 \mathrm{mM}$ Tris-Cl (pH6.8)

$6 \%$ SDS

$0.3 \%$ Bromophenol Blue

$30 \%$ Glycerol

$300 \mathrm{mM} \beta$-Mercaptoethanol

10 x SDS Run Buffer (per Litre)

$144 \mathrm{~g}$ Glycine

$30.3 \mathrm{~g}$ Tris

$10 \mathrm{~g}$ SDS

Coomassie Blue Stain (per Litre)

$2.5 \mathrm{~g} / \mathrm{L}$ Coomassie Brilliant Blue

$450 \mathrm{~mL} 100 \%$ Ethanol

$100 \mathrm{~mL} 100 \%$ Acetic Acid

$450 \mathrm{~mL}$ dd $2 \mathrm{O}$

Destain (per Litre)

$400 \mathrm{~mL} 100 \%$ Methanol

$100 \mathrm{~mL} 100 \%$ Acetic Acid

$500 \mathrm{~mL}$ ddH $2 \mathrm{O}$ 


\subsection{Assays for NTR activity with nitroaromatic substrates}

\subsubsection{SOS assay}

\subsubsection{SOS assay using a lacZ reporter}

Individual 96 microplate wells containing $200 \mu \mathrm{L} \mathrm{LB}+$ Amp + glucose $(0.4 \%)$ were inoculated from glycerol stocks with SOS-R2 pUCX:ntr strains and grown overnight $(\sim 16 \mathrm{~h})$ at $30^{\circ} \mathrm{C}, 200 \mathrm{rpm}$. The next morning $15 \mu \mathrm{L}$ of each overnight culture was used to inoculate $200 \mu \mathrm{L}$ of assay media ( $\mathrm{LB}+\mathrm{Amp}+0.05 \mathrm{mM}$ IPTG $+0.2 \% \mathrm{v} / \mathrm{v}$ glucose) in a 96 well microplate and cultures were grown for $3.5 \mathrm{~h}$ at $30^{\circ} \mathrm{C}, 200 \mathrm{rpm}$. Cultures were then split, with $100 \mu \mathrm{L}$ aliquots inoculated into two fresh microplates, one containing 100 $\mu \mathrm{L}$ assay media + drug at $2 \times$ challenge concentration and one containing $100 \mu \mathrm{L}$ assay media + DMSO control. The $\mathrm{OD}_{600}$ absorbance for each well was determined using an EnSpire 2300 Multilabel Reader (Perkin Elmer, Waltham, MA, USA). The plate was returned to $30{ }^{\circ} \mathrm{C}, 200 \mathrm{rpm}$ for 3-4 h prior to again measuring culture $\mathrm{OD}_{600}$ followed by quantification of $\beta$-galactosidase induction. This was accomplished by removing a $20 \mu \mathrm{L}$ cell aliquot to $130 \mu \mathrm{L}$ of ONPG assay buffer (see below) and incubating the plate for 1530 minutes without shaking at $37{ }^{\circ} \mathrm{C}$ until sufficient colour development as a result of ONPG cleavage. This reaction was then halted by addition of $50 \mu \mathrm{L}$ of $1 \mathrm{M} \mathrm{Na}_{2} \mathrm{CO}_{3}$ to each well. Aborbance readings at $420 \mathrm{~nm}$ and $550 \mathrm{~nm}$ were taken and used to calculate Miller units using the Miller equation (Miller, 1972). Any drug dependent growth inhibition was monitored by comparing the $\mathrm{OD}_{600}$ increase for drug challenged and unchallenged cultures.If growth inhibition was observed for any over expression strains assays were repeated at lower compound concentrations.

\section{ONPG assay buffer:}

Fresh ONPG assay buffer was made up prior to each use. This consisted of $9 \mathrm{~mL} 50 \mathrm{mM}$ $\mathrm{NaPO}_{4}$ buffer (pH 7.0), 4 mL Z-buffer and $1 \mathrm{~mL}$ T-base per 96 well microplate assayed.

\section{Z-buffer}

$\mathrm{NaH}_{2} \mathrm{PO}_{4} 0.074 \mathrm{M}$

$\mathrm{Na}_{2} \mathrm{HPO}_{4} 0.126 \mathrm{M}$

$\mathrm{MgSO}_{4} 2 \mathrm{mM}$

$\mathrm{MnSO}_{4} 0.4 \mathrm{mM}$

CTAB 399 mg.L ${ }^{-1}$

Sodium deoxycholate $199.5 \mathrm{mg} . \mathrm{L}^{-1}$ 
$\beta$-mercaptoethanl $0.174 \mathrm{M}^{*}$

\section{$\underline{\text { T-base }} * *$}

$\mathrm{K}_{2} \mathrm{HPO}_{4} 0.08 \mathrm{M}$

$\mathrm{KH}_{2} \mathrm{PO}_{4} 0.044 \mathrm{M}$

$\left(\mathrm{NH}_{4}\right)_{2} \mathrm{SO}_{4} 15.1 \mathrm{mM}$

Tri-sodium citrate 1 g. $\mathrm{L}^{-1}$

ONPG 8 mg.mL $\mathrm{m}^{-1}$

*added to assay buffer immediately prior to use, works out to be $52 \mu \mathrm{L}$ per 96 well microplate

** Post ONPG addition stored in single use aliquots at $-20{ }^{\circ} \mathrm{C}$

\subsubsection{SOS assay using a GFP reporter}

Cultures of 7KO pGFP pUCX:ntr were inoculated from glycerol stocks and grown overnight $(\sim 16 \mathrm{~h})$ in 96 well plates containing $200 \mu \mathrm{L} \mathrm{LB}+\mathrm{Amp}+\mathrm{Spec}+0.4 \% \mathrm{v} / \mathrm{v}$ glucose at $30{ }^{\circ} \mathrm{C}, 200 \mathrm{rpm}$. The next morning $10 \mu \mathrm{L}$ of overnight cultures was used to inoculate $200 \mu \mathrm{L}$ of assay media $(\mathrm{LB}+\mathrm{Amp}+\mathrm{Spec}+0.05 \mathrm{mM} \mathrm{IPTG}+0.2 \% \mathrm{v} / \mathrm{v}$ glucose) in a 96 well microplate and cultures were grown for $3 \mathrm{~h}$ at $30{ }^{\circ} \mathrm{C}, 200 \mathrm{rpm}$. Cultures were then split, with $100 \mu \mathrm{L}$ aliquots inoculated into two fresh microplates, one containing $100 \mu \mathrm{L}$ assay media + drug at $2 \times$ challenge concentration and one containing $100 \mu \mathrm{L}$ assay media + DMSO control. OD $_{600}$ absorbance for each well was determined and the plate was returned for further incubation at $30{ }^{\circ} \mathrm{C}, 200 \mathrm{rpm}$. At approprite time intervals (generally 4-6 h) culture $\mathrm{OD}_{600}$ and GFP-mut3 fluorscence readings (ex/em $488 / 510 \mathrm{~nm}$ ) were recorded and SOS induction determined by dividing raw fluorescence of drug challenged cultures by that of unchallenged controls. Any drug dependent growth inhibition was monitored for by comparing the $\mathrm{OD}_{600}$ increase of drug challenged and unchallenged cultures.

\subsubsection{Growth inhibition assay}

Overnight cultures and initial NTR induction proceeded as per the lacZ SOS assay, with the assays diverging when strains came to be challenged with drugs. At this point $40 \mu \mathrm{L}$ aliquots of each strain were moved into a 384 microplate wherein wells contained either $40 \mu \mathrm{L}$ assay media $(\mathrm{LB}+\mathrm{Amp}+0.05 \mathrm{mM} \mathrm{IPTG}+0.2 \% \mathrm{v} / \mathrm{v}$ glucose $)+$ drug at $2 \mathrm{x}$ challenge concentration or a media + DMSO only control. In each experiment strains were assayed in duplicate from seperate day cultures. The density of the cultures $\left(\mathrm{OD}_{600}\right)$ was measured, the plate returned to $30{ }^{\circ} \mathrm{C}, 200 \mathrm{rpm}$ for $4 \mathrm{~h}$ and then $\mathrm{OD}_{600}$ measured 
again. Relative increases in $\mathrm{OD}_{600}$ for challenged vs unchallenged wells were then used to calculate percentage growth inhibition.

\subsubsection{IC 50 assay}

Overnight cultures were grown as per the lacZ SOS assay. The next morning $50 \mu \mathrm{L}$ of each culture was used to inoculate $1 \mathrm{~mL}$ of assay media (LB + Amp + $0.05 \mathrm{mM} \mathrm{IPTG} \mathrm{+}$ $0.2 \% \mathrm{v} / \mathrm{v}$ glucose) in separate $15 \mathrm{~mL}$ Falcon tubes. These Falcons were incubated at 30 ${ }^{\circ} \mathrm{C}, 200 \mathrm{rpm}$ for $2.5 \mathrm{~h}$ before $40 \mu \mathrm{L}$ aliquots of each culture were added to wells of a 384 well microplate containing $40 \mu \mathrm{L}$ of assay media $\pm 2 \times$ final drug concentration. Each culture was exposed, in duplicate, to at least seven wells containing a two-fold increasing tiration of drugs and one media only control. Media was supplemented with DMSO as appropriate and DMSO in media never exceeded 4\%. Percentage growth inhibition at each drug concentration was determined as per the growth inhibiton assay and IC50 values (the drug concentration which caused 50\% strain growth inhibition) were calculated using non-linear regression analysis in SigmaPlot, fitting the data to a 4-parameter logistics curve. (Systat Software, Richmond, CA, USA).

\subsubsection{NAD(P)H depletion assay}

Overnight cultures and initial NTR induction proceeded as per the lacZ SOS assay. Cultures were then incubated at $30^{\circ} \mathrm{C}, 200 \mathrm{rpm}$ for $6 \mathrm{~h}$. Crude cell lysates were prepared by mixing cultures with BugBuster® Protein Extraction Reagent in a 1:1 (v/v) ratio at room temperature for $30 \mathrm{~min}$, lysates were then diluted as appropriate using $100 \mathrm{mM}$ $\mathrm{KPO}_{4}$ buffer $\mathrm{pH}$ 8.0. To monitor cell lysate cofactor consumption, replicate $200 \mu \mathrm{l}$ reactions consisting of $0.25 \%$ DMSO, $100 \mathrm{mM} \mathrm{KPO}_{4}$ buffer $\mathrm{pH} 8.0,50 \mu 1$ diluted crude cell lysate $\pm 150 \mu \mathrm{M}$ substrate were prepared and the reactions initiated by addition of 50 $\mu \mathrm{L}$ of $800 \mu \mathrm{M}$ NAD(P)H. Reactions were halted by addition of $50 \mu \mathrm{l}$ NBT solution (2

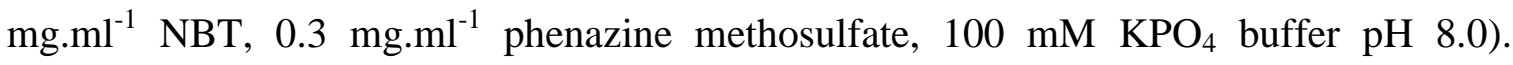
Phenazine methosulfate was mixed with NBT immediately prior to use. Levels of formazan production, corresponding to the amount of $\mathrm{NAD}(\mathrm{P}) \mathrm{H}$ remaining in each well, were quantified by measuring absorbance at $590 \mathrm{~nm}, 3-5 \mathrm{~min}$ post NBT addition. The range of activities able to monitored by the assay were controlled both by the dilution factor of the crude cell lysate and by varying the length of time for which the oxidoreductase reactions were allowed to proceed. 


\subsubsection{NTR activation of fluorogenic probes in $E$. coli}

Cultures of SOS-R2 or 7KO-pGFP were grown as per the lacZ or GFP SOS assay respectively until the point of prodrug challenge, whereupon cultures were instead treated with fluorogenic probe containing media to a final concentration of $25 \mu \mathrm{M}$. Cultures were returned to the incubator $\left(30{ }^{\circ} \mathrm{C}, 200 \mathrm{rpm}\right)$ and $30 \mathrm{~min}$ to $2 \mathrm{~h}$ later fluorescence determined with appropriate excitation and emission wavelengths using the EnSpire 2300 Multilabel Reader (Perkin Elmer, Waltham, MA, USA).

\subsection{Fluorescence activated cell sorting of mutant libraries}

Overnight cultures were set up by thawing one $200 \mu \mathrm{L}$ aliquot of a of $7 \mathrm{KO}$ pGFP pUCX:ntr mutant library (generation described in section 2.9.1) and using this to inoculate 10 wells of a 96 well microplate, each filled with $200 \mu \mathrm{L}$ of $\mathrm{LB}+\mathrm{Amp}+\mathrm{Spec}$ $+0.4 \% \mathrm{v} / \mathrm{v}$ glucose. These were then grown overnight at $30{ }^{\circ} \mathrm{C}, 200 \mathrm{rpm}$. The next morning cultures from these wells were pooled and $2 \times 10 \mu \mathrm{L}$ aliquots used to inoculate $200 \mu \mathrm{L}$ apeice of assay media (LB + Amp + Spec $+0.05 \mathrm{mM} \mathrm{IPTG} \mathrm{+} \mathrm{0.2 \%} \mathrm{v/v} \mathrm{glucose)}$ in a 96 well microplate. Cultures were then grown, split and challenged with $1 \mu \mathrm{M}$ RB6145 as described in the standard GFP based SOS assay (Section 2.14.1.2). After $4 \mathrm{~h}$ incubation with $1 \mu \mathrm{M}$ RB6145 the 2 x $200 \mu \mathrm{L}$ of drug challenged culture was collected, pooled, spun down in a microcentrifuge $(13,000 \mathrm{rpm}, 30 \mathrm{~s})$, and the cells washed three times in phosphate buffered saline (PBS, pH 7.0) resuspended in $1000 \mu \mathrm{L}$ PBS then diluted (50 $\mu \mathrm{L}$ cells into $1000 \mu \mathrm{L}$ PBS) in FACS tubes. Samples were analysed on a FACS Vantage SE-Diva (Becton Dickinson Biosciences, Bedford, MA) with GFP-mut3 fluorescence excited using a $100 \mathrm{~mW}$ blue laser (488 nm) and detected using a photomultiplier tube with a 530/30 nm band pass filter. Gates for sorting were set using a culture of 7KO pGFP NfsA_Ec cells challenged with $3 \mu \mathrm{M}$ RB6145 (positive fluorescence) or unchallenged controls (negative fluorescence). Sorted events were collected, spun down in a microcentrifuge tube and then resuspended in $1 \mathrm{~mL}$ of $\mathrm{LB}+$ $\mathrm{Amp}+\mathrm{Spec}+0.4 \% \mathrm{v} / \mathrm{v}$ glucose and allowed to recover at $37^{\circ} \mathrm{C} 200 \mathrm{rpm}$ for $1 \mathrm{~h}$ before plating in $100 \mu \mathrm{L}$ aliquots on solid media plates containing Spec + Amp antibiotics. Plates were grown overnight at $37{ }^{\circ} \mathrm{C}$ in a static incubator, before colonies were picked individually into separate media filled wells of 96 well microplates. These plates were grown overnight, mixed with glycerol and then stored at $-80{ }^{\circ} \mathrm{C}$ until required to inoculate day cultures for screening activity with nitroaromatic substrates. 


\subsection{Docking}

This modelling work was carried out in conjunction with Dr Jack Flanagan at the ACSRC. Molecular docking was performed using the X-ray crystal structure of NfsB_Ec (PDB code 1DS7). In preparing the structures for docking, waters were removed and the structures protonated using SYBYL8.0.3 (TRIPOS). After visual inspection of the crystal structure the orientation of Asn117 from chain A was manually flipped $180{ }^{\circ} \mathrm{C}$ to give a favourable interaction with nearby Arg120 and Glu101 residues (this orientation was already seen in chain B). SN33623 and CB1954 were docked into the NfsB_Ec active site using GOLD v5.1, with a docking cavity of $12 \AA$ centred on N5 of the FMN co-factor. The docking was performed using the ChemPLP scoring function, with 20 poses per ligand saved. Search efficiency was set at $200 \%$, and diverse solutions were allowed with $1.5 \AA$ freedom. One $\mathrm{H}$ bond constraint was set to the hydrogen of the backbone amide of Thr41, as this bond was found in the crystal structure of CB1954 bound in NfsB_Ec ((Johansson et al., 2003) PDB code 1IDT). In regard to CB1954 docking the solutions were visually inspected for poses which recapitulated CB1954 binding as seen in the Xray crystal structure 1IDT. The poses returned for SN33623 docking were visually inspected for solutions which showed favourable confirmation of the amide bond within SN33623 and positioned the $\mathrm{NO}_{2}$ functional group of the ligand directly over the FMN cofactor.

\subsection{Generation, maintenance and storage of HCT-116:NTR cell lines}

\subsubsection{HCT-116 maintenance}

HCT-116 cell lines were, unless otherwise stated, maintained in $\alpha$-MEM (Gibco, Grand Island, NY) media supplemented with 5\% Fetal Calf Serum (FCS) and grown in monolayer up to $80 \%$ confluency in a humidified incubator $\left(37{ }^{\circ} \mathrm{C}, 5 \% \mathrm{CO}_{2}\right)$. Cells were sub-cultured at least every 7 days, and kept in culture for $<3$ months post growth from frozen stocks confirmed to be mycoplasma free by PCR-ELISA (Roche Diagnostics Mannheim, Germany). Cell lines carrying a F279-V5 plasmid were maintained in media supplemented with $1 \mu \mathrm{M}$ puromycin.

When required, cells were harvested from growth flasks and microplates using a standard Trypsin/EDTA protocol. Briefly growth media was aspirated and cells washed once with $1 \times$ PBS, then warm $0.05 \%$ Trypsin/EDTA (Invitrogen Corporation, Carlsbad, CA, USA) sufficient to cover flask/well surface and left until cells detached (typically $<5 \mathrm{~min}$ ). 
Trypsin was inactivated by re-suspending the cells in FCS containing media and a cell aliquot taken to determine cell count using a Z2 Coulter Particle Count and Cell Analyzer, (Beckman Coulter ${ }^{T M}$, Fullerton, CA, USA). If required for sub-culture, the cell volume necessary for cell maintenance was transferred to a new flask containing fresh media.

\subsubsection{Storage and recovery from frozen stocks}

For long term storage cells were harvested from T175 flasks, centrifuged for $5 \mathrm{~min}$ at 300 $\mathrm{RCF}$, the media discarded and cells resuspended to a concentration of $1-2 \times 10^{6}$ cells. $\mathrm{mL}^{-1}$ in a solution of FCS containing 10\% DMSO. The cell mix was placed in $1 \mathrm{~mL}$ aliquots in cryotubes and then frozen in $1{ }^{\circ} \mathrm{C}$ increments using a Mr. Frosty® Cryo $1{ }^{\circ} \mathrm{C}$ Freezing Container (Thermo Fisher Scientific; Waltham, MA, USA). When a temperature of $-80{ }^{\circ} \mathrm{C}$ was reached the cryotubes were placed in liquid nitrogen for long term storage.

Cell recovery was accomplished by removal of cell aliquots from nitrogen storage, thawing in a $37{ }^{\circ} \mathrm{C}$ water bath and rapid addition of thawed cells to a T175 flask containing $40 \mathrm{~mL}$ of pre warmed $\alpha \mathrm{MEM}+5 \% \mathrm{FCS}$.

\subsubsection{Generating F279-V5:ntr construct using Gateway® technology}

\subsubsection{Generating Gateway ${ }^{\circledR}$ compatible PCR products}

NTR genes to be transfected were amplified using Gateway® compatible primers (see Table 2-1). These introduced a mammalian Kozak consensus sequence, a Shine-Dalgarno prokaryote consensus sequence and Invitrogen Gateway® BP recombination sites, as well as an extra GGC (glycine) codon added to each gene immediately after the ATG start codon to ensure a uniform ATGG four-base start sequence, and conversion of all stop codons to TAG. PCR products were confirmed to be of correct size using gel electrophoresis and purified using the DNA Clean and Concentrator ${ }^{\mathrm{TM}}-5$ Kit (Zymo Research; Irvine, CA, USA).

\subsubsection{BP clonase}

Recombination of the PCR product into an entry vector, pDONR ${ }^{\mathrm{TM}} 221$ (Invitrogen Corporation, Carlsbad, CA, USA) was accomplished using Gateway ${ }^{\circledR}$ BP Clonase ${ }^{\mathrm{TM}}$ II enzyme mix (Invitrogen). $150 \mathrm{ng}$ of pDONR221 was mixed with 20-50 fmol of PCR product, $1 \mu \mathrm{L}$ of BP Clonase enzyme mix and TE $\mathrm{pH} 8.0$ buffer to $10 \mu \mathrm{L}$ and incubated at $25{ }^{\circ} \mathrm{C}$ for $14 \mathrm{~h} .1 \mu \mathrm{l}$ of the $2 \mu \mathrm{g} . \mu \mathrm{l}^{-1}$ Proteinase $\mathrm{K}$ solution supplied with the kit was added 
to the mixture which was left for a further $10 \mathrm{~min}$ at $37^{\circ} \mathrm{C}$. The resulting mix was transformed into E. coli cloning strain $\mathrm{DH} 5 \alpha$, and plated on LB + Kan agar plates. Colonies were grown and analysed for insertion of NTR sequence by restriction enzyme digest with NheI.

\subsubsection{LR Clonase}

Recombination of the NTR construct from pDONR221 into the ACSRC constructed mammalian expression vector F279/V5 (Prosser et al., 2013) was accomplished using Gateway ${ }^{\circledR}$ LR Clonase ${ }^{\mathrm{TM}}$ II enzymes mix (Invitrogen Corporation, Carlsbad, CA, USA). $150 \mathrm{ng}$ of pDONR221:ntr was mixed with150 ng of F279/V5, $1 \mu \mathrm{L}$ of LR Clonase enzyme mix and TE pH 8.0 buffer to $10 \mu \mathrm{L}$ and incubated at $25^{\circ} \mathrm{C}$ for $14 \mathrm{~h} .1 \mu \mathrm{l}$ of the $2 \mu \mathrm{g} . \mu \mathrm{l}^{-1}$ Proteinase $\mathrm{K}$ solution was added to the mixture which was left for a further $10 \mathrm{~min}$ at $37^{\circ} \mathrm{C}$. The resulting mix was transformed into E. coli cloning strain $\mathrm{DH} 5 \alpha$, and plated on LB + Amp agar plates. Colonies were grown and analysed for insertion of NTR sequence by restriction enzyme digest with HindIII. Correct insertion was confirmed by DNA sequencing of positive constructs.

\subsubsection{DNA isolation}

DNA for transfection was prepared using the Compact Prep® Plasmid Midi Kit (Qiagen, $\mathrm{GmbH}$, Germany) according to manufacturer's instructions. Prior to transfection midiprep samples were cleaned using a standard ethanol precipitation protocol (Sambrook and Russell, 2001).

\subsubsection{Transfection of HCT-116 cells}

Stable HCT-116:ntr cell lines described in this thesis were transfected with F279-V5 plasmids using one of the following protocols.

\subsubsection{FuGENE 6/X-tremeGENE HP transfection}

150,000 cells in $4 \mathrm{~mL}$ media were seeded into each well of a 6 well plate and incubated over night for $24 \mathrm{~h}$. For every well to be transfected a Falcon tube was set up containing $2 \mu \mathrm{g}$ of the relevant plasmid DNA diluted in $200 \mu \mathrm{L}$ of $\alpha$-CMEM, not supplemented with FCS. Room temperature transfection reagent $(2-8 \mu \mathrm{L}$ per tube) was added directly to Falcon tube media, mixed gently and incubated at room temperature for 20- $30 \mathrm{~min}$. Transfection reagent was either FuGENE 6 or X-tremeGENE HP DNA Transfection reagent (both Roche Diagnostics GmBH, Mannheim, Germany). The transfection/DNA mix was then added drop wise to each well, mixed gently by rocking and the plate 
returned to the incubator. For each strain to be generated 2-6 wells of cells were treated with transfection/DNA mixes and cells later pooled. 2-3 days post transfection media was aspirated and replaced with media supplemented with $1 \mu \mathrm{M}$ puromycin. Cell growth was then inspected daily using a microscope and media containing dead cells aspirated and replaced as required, with puromycin concentration in the media gradually increased to $3 \mu \mathrm{M}$. When cells reached sufficient quantity they were harvested and maintained in T75-T175 flasks. This was continued for 2-4 weeks until all cells displayed puromycin resistance and cell populations had grown to a sufficient quantity to both freeze cell-line samples for long term storage and maintain the cells in subculture.

\subsubsection{Lipofectamine® LTX transfection}

500,000 cells in $2 \mathrm{~mL}$ media were seeded into each well of a 6 well plate and incubated over night for $24 \mathrm{~h}$. Media was aspirated and replaced with fresh media. For every well to be transfected a Falcon tube was set up containing 2.5-3.75 $\mu \mathrm{g}$ of the relevant plasmid DNA diluted in $500 \mu \mathrm{L}$ of OptimMem (reduced serum) (Gibco). Plus ${ }^{\mathrm{TM}}$ transfection reagent (2.5-3.75 $\mu \mathrm{L}$ per tube) was added directly to Falcon tube media, mixed gently and incubated at room temperature for $5 \mathrm{~min}$ followed by addition of $7.5-15 \mu \mathrm{L}$ of Lipofectamine ${ }^{\circledR}$ LTX transfection reagent (Invitrogen). This was gently mixed, incubated at room temperature for $30 \mathrm{~min}$ and then added drop wise to cells. Generation of stable cell lines then proceeded as in section 1.18.2.1.

\subsubsection{Transient transfection}

In some instances nitroreductase expression and activity was assessed following transient transfection. This was accomplished using identical initial transfection protocols, but $\sim 24$ $\mathrm{h}$ post transfection the cells, which comprised a mixed population of those which had or had not taken up the F279-V5 plasmid, were collected and used in subsequent protocols.

\subsection{Western blots}

\subsubsection{Sample collection and quantification}

Protein was extracted from $1 \times 10^{6}$ cells. These were adhered in the well of a 6 well plate, rinsed once with cold PBS and lysed by exposure to $250 \mu \mathrm{L}$ Radio Immuno Precipitation Assay (RIPA) buffer for 30 min on ice. Lysates were removed from the wells and spun in a microcentrifuge for $15 \mathrm{~min}$ at $4{ }^{\circ} \mathrm{C}, 13,000 \mathrm{rpm}$ to pellet remaining cell debris. The clear lysate was removed to a new tube and stored at $-20{ }^{\circ} \mathrm{C}$ until required. 
Sample protein concentration was determined using a Bicinchoninic Acid (BCA) Protein Assay. Briefly in a 96 well microplate protein samples were diluted 1:3 in $0.1 \mathrm{M} \mathrm{NaOH}$ and mixed with $100 \mu \mathrm{L}$ of BCA reagent (prepared from mixing $1 \times 4 \%$ of $\mathrm{CuSO}_{4}$ with 50 $\mathrm{x}$ BCA). The plate was incubated at room temperature with gentle agitation for 10-15 minutes and optical absorbance at $562 \mathrm{~nm}$ read. Sample protein concentration was determined by comparison to identically prepared protein standards of BSA spanning in a serial dilution from $0-1000 \mu \mathrm{g} / \mathrm{mL}$.

\section{RIPA Buffer}

Tris $\mathrm{HCl} 50 \mathrm{mM} \mathrm{pH} 8.0$

$\mathrm{NaCl} 150 \mathrm{mM}$

EDTA $1 \mathrm{mM}$

Na Deoxycholate $0.5 \%$

SDS $0.1 \%$

$\beta$-mercaptoethanol 5\%

Protease inhibitor cocktail 1\% (Sigma-Aldrich, St. Louis, MO,USA)

$1 \%(\mathrm{v} / \mathrm{v})$ Nonidet P40 (Roche Diagnostics, Indianapolis, IN, USA)

\subsubsection{Gel electrophoresis separation and transfer of proteins}

Protein samples were thawed and diluted in RIPA buffer to allow for loading of $20 \mu \mathrm{g}$ of protein per well. Samples were mixed with $3 \mathrm{x}$ SDS loading buffer and incubated at $98{ }^{\circ} \mathrm{C}$ for 4 min. Samples were run on $1.5 \mathrm{~mm}$ thick, 10-15 well, NuPAGE® 4-12\% Bis-Tris gels (Invitrogen Corporation, Carlsbad, CA, USA) using a XCell SureLock ${ }^{\mathrm{TM}}$ Electrophoresis Mini-Cell system (Invitrogen) filled with NuPAGE® MES SDS Running Buffer. One lane was loaded with $5 \mu \mathrm{L}$ of Bio-Rad pre-stained MW marker to determine protein size. Proteins were separated by running electrophoresis at $150 \mathrm{~V}$ for $90 \mathrm{~min}$. Following this the gel cast was dissembled and protein transfer onto nitrocellulose membrane (Bio-Rad Trans-Blot ${ }^{\circledR}$ Transfer Medium) accomplished using the Bio-Rad Mini Trans-Blot ${ }^{\circledR}$ Electrophoretic Transfer Cell system filled with ice cold Transfer Buffer with electrophoresis run at $100 \mathrm{~V}$ for $70 \mathrm{~min}$.

Prior to antibody staining the membrane was blocked in Blocking Buffer with gentle agitation for $60 \mathrm{~min}$. Blocking solution was removed by decanting and membrane was washed by soaking in PBS-T (PBS, Tween-20 0.05\%) for 5 min and decanting 3 times.

\section{Transfer Buffer}

Glycine 14.4 g.L. ${ }^{-1}$

Tris Base 3 g.L ${ }^{-1}$

Methanol 20\% v/v 
MQ

\section{Blocking Buffer}

PBS

Tween-20 0.05\%

Milk powder 5\%

\subsubsection{Antibody detection of proteins}

Incubation with primary antibody was carried out in Blocking Buffer at $4{ }^{\circ} \mathrm{C}$ overnight with gentle agitation at concentrations as stated in Table 2-11. Membranes which required secondary antibody staining were decanted, washed 3 times for $5 \mathrm{~min}$ in PBS-T and the appropriate secondary antibody added diluted in Blocking Buffer and incubated with the membrane at room temperature for $2 \mathrm{~h}$. Following $3 \times 5$ min washes in PBS-T the membranes were incubated with SuperSignal ${ }^{\circledR}$ West Pico Chemiluminescent Substrate (Thermo Fisher Scientific; Waltham, MA, USA) for $5 \mathrm{~min}$ with gentle agitation. Image acquisition was accomplished using a Fujifilm LAS-4000 (Fujifilm, Tokyo, Japan). Protein expression was quantified using ImageJ (version 1.46 of the public domain Java image processing program) and normalised against $\beta$-actin levels. As this required sequential staining of the same membrane with two different primary/secondary antibodies, the membranes were stripped using Restore ${ }^{\mathrm{TM}}$ western blot stripping buffer (Thermo Fisher Scientific; Waltham, MA, USA) following collection of the $\beta$-actin image.

\begin{tabular}{ll}
\hline Antibody & Dilution \\
\hline Mouse $\alpha$-actin & $1: 10,000$ \\
Sheep $\alpha$-NfsA & $1: 20,000$ \\
Sheep $\alpha$-NfsB & $1: 20,000$ \\
Goat $\alpha$-mouse IgG-HRP & $1: 10,000$ \\
Rabbit $\alpha$-sheep IgG-HRP & $1: 10,000$ \\
\hline
\end{tabular}

Table 2-11 Antibody dilutions for use in western blots

Sheep anti-NfsB serum was raised against purified NfsB by South Pacific Sera (Timaru, New Zealand). The NfsA antibody was a generous gift from Dr Peter Searle (University of Birmingham). Mouse anti-actin antibody was purchased from Millipore (Merck Millipore, MA, USA) and Goat and Rabbit secondary antibodies from Santa Cruz Biotechnology, Inc (Santa Cruz Biotechnology, Inc., TX, USA). 


\subsection{Analysis of activation of fluorogenic probes by HCT-116 cell lines}

\subsubsection{FLoid® Cell Imaging Station}

Cells from each HCT-116:ntr expression line to be assessed were harvested and 100,000300,000 cells seeded into individual wells of a 6 well plate and incubated with $1 \mathrm{~mL}$ media containing $50 \mu \mathrm{M}$ of fluorogenic probe for $2 \mathrm{~h}$. Images were taken using the preset channels (relief phase, blue fluorescence, green fluorescence, and red fluorescence) of a FLoid® Cell Imaging Station (Invitrogen Corporation, Carlsbad, CA, USA), with identical focus, exposure and overlay settings for each cell line when comparing activation of the same fluorophore.

\subsubsection{Flow cytometry}

Cells from each HCT-116:ntr expression line to be assessed were harvested and $0.5 \times 10^{6}$ cells in $450 \mu \mathrm{L}$ of media seeded into wells of a non-tissue culture treated 24 -well plate and returned to the incubator for $30 \mathrm{~min}$ to equilibrate to $37{ }^{\circ} \mathrm{C} .50 \mu \mathrm{L}$ of media containing the probe FSL 61 was added to each well to give a final concentration of 50 $\mu \mathrm{M}$ and the plate returned to the incubator for $2 \mathrm{~h}$. Cells were then moved into tubes for flow cytometry and placed on ice to halt probe metabolism before immediate analysis of the fluorescence of single cell events on a Becton Dickinson LSRII Flow Cytometer (Becton Dickinson Biosciences, Bedford, MA). Single cells were gated using forward- and side-scatter with a $488 \mathrm{~nm}$ laser. Fluorescence was excited using the $355 \mathrm{~nm}$ UV laser and fluorescence emission monitored at $450 \mathrm{~nm} \pm 25$.

\subsection{Analysis of EF5/SN33623 metabolism by HCT-116 cell lines}

Cells from each HCT-116:ntr expression line to be assessed were harvested and 1 x $10^{6}$ cells seeded into the well of a 6 well plate. Plates were returned to the incubator for 30 min to equilibrate to $37{ }^{\circ} \mathrm{C}$, appropriate volumes of EF5/SN33623 were then added to media and plates were incubated with imaging agent for 1- $2 \mathrm{~h}$. Cells were collected, spun down (300 RCF, $5 \mathrm{~min}$ ) and resuspended in $1 \mathrm{~mL} \mathrm{PBS}+4 \%$ paraformaldehyde for $1 \mathrm{~h}$ at $4{ }^{\circ} \mathrm{C}$ to fix cells. Paraformaldehyde was aspirated and cells were spun down and washed twice using $1 \mathrm{~mL}$ PBS, resuspended in $1 \mathrm{~mL}$ blocking solution (20\% non-fat milk, 5\% FCS, $0.3 \%$ Tween-20 and $1.5 \%$ albumin in PBS) and left overnight at $4{ }^{\circ} \mathrm{C}$. The next day cells were washed once using $1 \mathrm{~mL}$ PBS and the cell pellet resuspended in $100 \mu \mathrm{g} \cdot \mathrm{mL}^{-1}$ Alexa488-ELK5.1 antibody in antibody carrier (PBS, 0.3\% Tween-20, 1.5\% albumin). Post antibody addition, care was taken to protect samples from light and to use pre-cooled 
reagents. Cells were left overnight at $4{ }^{\circ} \mathrm{C}$. The next day prior to cell analysis cells were rinsed twice using $1 \mathrm{~mL}$ PBS $0.3 \%$ Tween-20 and once using $1 \mathrm{~mL}$ PBS before being resuspended in $500 \mu \mathrm{L}$ PBS and transferred to a FACS tube. Fluorescence of single cell events was monitored using a Becton Dickinson LSRII Flow Cytometer, with samples excited using the $488 \mathrm{~nm}$ (blue-green) laser and fluorescence emission monitored at 530 $\mathrm{nm} \pm 20$.

\subsection{Determining in vivo hypoxia dependant metabolism of EF5 and SN33623}

Where indicated methods outlined in this section were carried out by collaborators at the ACSRC.

\subsubsection{Animal handling}

All animal handling was conducted by Alex Mowday.

Specific pathogen-free homozygous nude NIH-III (Charles River Laboratories, Wilmington, MA, USA) mice were bred by the Vernon Jansen Unit (shared vivarium, University of Auckland). They were housed in Techniplast microisolator cages and fed Harlan Teklad diet 2018i. All animal studies were approved by the University of Auckland Animal Ethics Committee (approval CR830).

\subsubsection{Treatment of mice carrying HCT-116 tumours with imaging agents}

\section{All animal handling was conducted by Alex Mowday.}

Xenograft HCT-116 tumours were established by subcutaneous inoculation of $10 \times 10^{6}$ HCT-116 cells in $100 \mu \mathrm{L}$ FCS free media into the flank of male NIH-III nude mice. When tumours reached $\sim 10 \mathrm{~mm}$ diameter mice were given $60 \mathrm{mg} / \mathrm{kg}$ pimonidazole (in saline) by intraperitoneal injection. After $60 \mathrm{~min}$ they were also treated with either 60 mg.kg ${ }^{-1}$ EF5 (in PBS) or 60 mg. $\mathrm{kg}^{-1}$ SN33623 (in PBS) by intraperitoneal injection. After 120 min mice were culled and tumours excised and fixed in $10 \%$ formalin dissolved in PBS (48 h), transferred to $70 \%$ ethanol then embedded in paraffin wax. Tumour section were cut $(5 \mu \mathrm{M})$ and mounted onto glass slides.

\subsubsection{Immunohistochemical staining of tumour sections}

Paraffin was removed and tissue samples were rehydrated. This was accomplished by heating cells for $30 \mathrm{~min}$ at $58{ }^{\circ} \mathrm{C}$, followed by soaking slides in a number of steps at room 
temperature Soaking steps were $10 \mathrm{~min}$ in Xylene, repeating with fresh Xylene, 2 min in $100 \% \mathrm{EtOH}$, repeating in fresh $100 \% \mathrm{EtOH}, 2$ min in $95 \% \mathrm{EtOH}, 5$ min in $\mathrm{ddH}_{2} \mathrm{O}$ and 10 min in 0.01 M TBS (Tris buffered saline $\mathrm{pH}$ 7.4). Antigen retrieval was achieved by boiling samples in $10 \mathrm{mM}$ sodium citrate buffer $\mathrm{pH} 6$ for $20 \mathrm{~min}$. Slides were cooled in citrate buffer on ice for $10 \mathrm{~min}$ then washed once in TBS and washed 3 times for $5 \mathrm{~min}$ in TBS-T (TBS- 0.1\% Tween-20). Samples were blocked for $1 \mathrm{~h}$ in $10 \%$ goat serum then stained overnight at $4{ }^{\circ} \mathrm{C}$ with $40 \mu \mathrm{g} \cdot \mathrm{mL}^{-1}$ of anti-pimonidazole antibody FITCHypoxyprobe-1 (Chemicon® International, clone 4.3.11.3) diluted in 5\% goat serum TBS-T. The next day the antibody was removed by washing $3 \times 5$ min in TBS-T and the samples stained with $75 \mu \mathrm{g} \cdot \mathrm{mL}^{-1}$ of Cy5 conjugated ELK5.1 (anti-EF5/SN33623 antibody) for $5 \mathrm{~h}$ at $4{ }^{\circ} \mathrm{C}$, antibody in $5 \%$ goat serum in TBS-T. Slides were washed 2 times for $5 \mathrm{~min}$ in TBS-T and 1 time in TBS, mounted using Prolong Gold antifade reagent (Invitrogen) and sealed with nail polish.

As it has been exhaustively confirmed that this protocol does not result in cross-reactivity of the Hypoxyprobe-1 and ELK5.1 antibodies (Dr. Maria Abbattista personal communication) single antibody stain controls were not carried out for this study.

\subsubsection{Fluorescence microscopy}

\section{Operation of the confocal microscope was carried out by Dr. Maria Abbattista.}

Images were acquired and overlaid using a Zeiss LSM 710 confocal microscope (Carl Zeiss AG, Oberkocken, Germany) and Zen2010 software. FITC fluorescence was excited using an argon laser (Excitation/Emission 499/519) and Cy5 fluorescence using a HeNe663 laser (650/670 nm). 
Chapter 3: Evaluation of 11 E. coli oxidoreductase enzymes for metabolism of 2-Nitroimidazole imaging agents

\subsection{Introduction}

This research has been part of a larger collaborative effort between Dr. Ackerley's laboratory and researchers at the ACSRC, seeking to translate NTR based GDEPT to human clinical trial. This progression would be greatly aided by an ability to noninvasively image the NTR in both clinical and preclinical settings.

This chapter describes the evaluation of 11 phylogenetically diverse bacterial oxidoreductases of $E$. coli origin for their ability to reduce a number of 2-NI probes which had been originally developed for the clinical imaging of hypoxia. These agents can image hypoxia because they are each reduced to a cell entrapped form, via an oxygen sensitive reaction, catalysed by endogenous human NTRs. Areas of hypoxia are then identified by biopsy based immunohistochemistry or, if the agents are radiolabelled, by noninvasive PET imaging. The central premise of our study was that if it was possible to identify oxygen insensitive bacterial NTRs that reduce the same agents, only in a more efficient manner, then these 2-NI compounds could potentially be repurposed to act as PET reporter probes for noninvasive imaging of bacterial NTRs.

The first section of this chapter focuses on assessing the $11 \mathrm{E}$. coli oxidoreductases by screening in an E. coli over-expression system, by purified protein kinetics and by a microplate based colorimetric NAD(P)H depletion screen.

\subsubsection{Identification of diverse Escherichia coli nitroreductase candidates}

The lack of clinical success of NfsB/CB1954, coupled with the ubiquity and diversity of bacterial NTRs, suggests that a wider examination of the enzyme superfamily could discover NTRs better suited for GDEPT. As a first step in this examination the Ackerley laboratory cloned NfsB and 10 other diverse NTR candidates to create a small E. coli over-expression library (Prosser et al., 2010). These 11 enzymes were all of E. coli origin, with the intention being that these would be used as a base to guide later library expansion using homologues from different bacterial species.

$\mathrm{NfsB}$, the prototypical NTR in a GDEPT context, was included as a matter of course. However, where as NfsB has been the most studied NTR in a GDEPT context it is the 
enzyme NfsA which is typically described as the "major" E. coli NTR (Bryant et al., 1981; Roldán et al., 2008), based on its superior activity with the antibiotic nitrofurazone (structure depicted in Figure 6-8). Though NfsB and NfsA share little sequence identity they both function as similarly sized FMN dependent homodimers with appreciable substrate overlap (Zenno et al., 1996). Furthermore NfsA has been shown to sensitize HeLa cells to CB1954 (Barak et al., 2006) and while our study was in progress, was further characterised by another group in a GDEPT context (Vass et al., 2009). A third enzyme included was $\mathrm{YieF}$, an $\mathrm{NAD}(\mathrm{P}) \mathrm{H}$ dependent oxidoreductase initially described for its ability to reduce chromate (Ackerley et al., 2004) included as a YieF mutant initially evolved for improved chromate reduction also shows activity with CB1954 (Barak et al., 2006).

A further four enzymes were chosen based on reported nitroreductase activity or annotation as NTRs in online databases. The first was NemA, originally identified due to its ability to reduce $N$-ethylmaleimide but added to the library as it can reduce trinitrotoluene (TNT) (Williams et al., 2004). A second library enzyme, AzoR, is so named for its ability to reductively cleave azo bonds $(-\mathrm{N}=\mathrm{N}-)$, a property of potential use in the degradation of a number of recalcitrant synthetic commercial dyes which contain this functional group (Nakanishi et al., 2001). In addition to this a close homologue from Rhodobacter sphaeroides has proven nitroreductase activity (Liu et al., 2007) again with TNT and various derivatives. Two other enzymes, YcdI (Loh et al., 2006) and YdjA (Choi et al., 2008), were annotated in the NCBI database as nitroreductase enzymes, though to the best of our knowledge no experimental data supporting these annotations have ever been published.

The remaining enzymes were chosen due to sequence or structural similarity to known CB1954 reducing enzymes. KefF was included as the closest $E$. coli orthologue of the CB1954-reducing NTR YwrO from B. amyloliquefaciens (40\% identity across 162 residues). YcaK was a hypothetical protein included based on its homology to the human enzyme NQO1 (44\% identity over a 127 amino acid stretch). The last two enzymes WrbA (Patridge and Ferry, 2006) and MdaB (Adams and Jia, 2006) were quinone oxidoreductases that have been observed to share structural similarity to NQO1 (Adams and Jia, 2006; Carey et al., 2007). In addition, MdaB (“modulator of drug activity B") was one of two enzymes discovered in a screen for proteins whose over-expression 
protected E. coli from a nitro-containing bis-naphthalimide drug DMP 840 (the other being NfsA, in this work termed MdaA) (Chatterjee and Sternberg, 1995).

To characterise all NTR candidates and to validate screening strategies for activation of DNA damaging nitroaromatic prodrugs these enzymes were first assessed for activity with CB1954 (Prosser et al., 2010). This allowed for adaption and optimisation of a tool called the SOS assay, a 96-well microplate screen for detecting nitroaromatic prodrug activation by NTRs over-expressed in E. coli.

\subsubsection{SOS assay}

To efficiently assess a large number of NTR candidates a simple, accurate and rapid screening system is needed. To achieve this the Ackerley laboratory had previously developed and optimised a microplate based assay to detect NTR catalysed activation of DNA damaging prodrugs (Prosser et al., 2010). The basis of this assay was the SOS assay, first developed to assess potential genotoxins by Quillardet et al. (1982).

This assay takes advantage of the SOS response, an endogenous process triggered by DNA damage in E. coli. It exists to promote the survival of DNA damaged E. coli by stalling cell division, enabling DNA repair and upregulating error prone polymerases to enable replication to proceed in the presence of DNA lesions. The SOS genes that are involved in this process are controlled by a dimeric transcriptional repressor termed LexA. LexA binds to the "SOS box", a palindromic DNA motif found in the promoter regions of these genes; and with its binding prevents access of RNA polymerase. The "trigger" of SOS induction is single stranded DNA (ssDNA) which can arise during repair of DNA lesions or at replication forks stalled due to DNA damage. The ssDNA binds to a protein called RecA, creating an active nucleoprotein filament which promotes autocatalytic cleavage of LexA thus releasing the repression of SOS genes.

This response can be quantitatively detected in an assay format by placing transcription of a reporter gene, typically either lacZ (Prosser et al., 2010; Quillardet et al., 1982; Shapiro and Baneyx, 2002) or the gene for GFP (Norman et al., 2005; Pennington and Rosenberg, 2007) under control of the promoter of an SOS induced gene. This is often (though not exclusively) sfiA (also known as sulA) (Norman et al., 2005) a gene induced late in the SOS response and involved in stalling cell division. In our adaptation of this test, cultures

of E. coli over-expressing individual NTR candidates are exposed to sub-lethal 
concentrations of a nitroaromatic prodrug. If NTR catalysed reduction occurs, the prodrug's latent DNA damaging toxicity is activated. The strains of E. coli we use for this assay contain chromosomally-integrated lacZ under the control of a sfiA promoter. The lac $Z$ gene encodes the enzyme $\beta$-galactosidase which cleaves the reporter substrate ortho-nitrophenyl galactoside (ONPG) to yield galactose and the yellow coloured onitrophenol, which directly correlates to levels of DNA damage present, and thus the activity of the prodrug activating NTR (Figure 3-1).

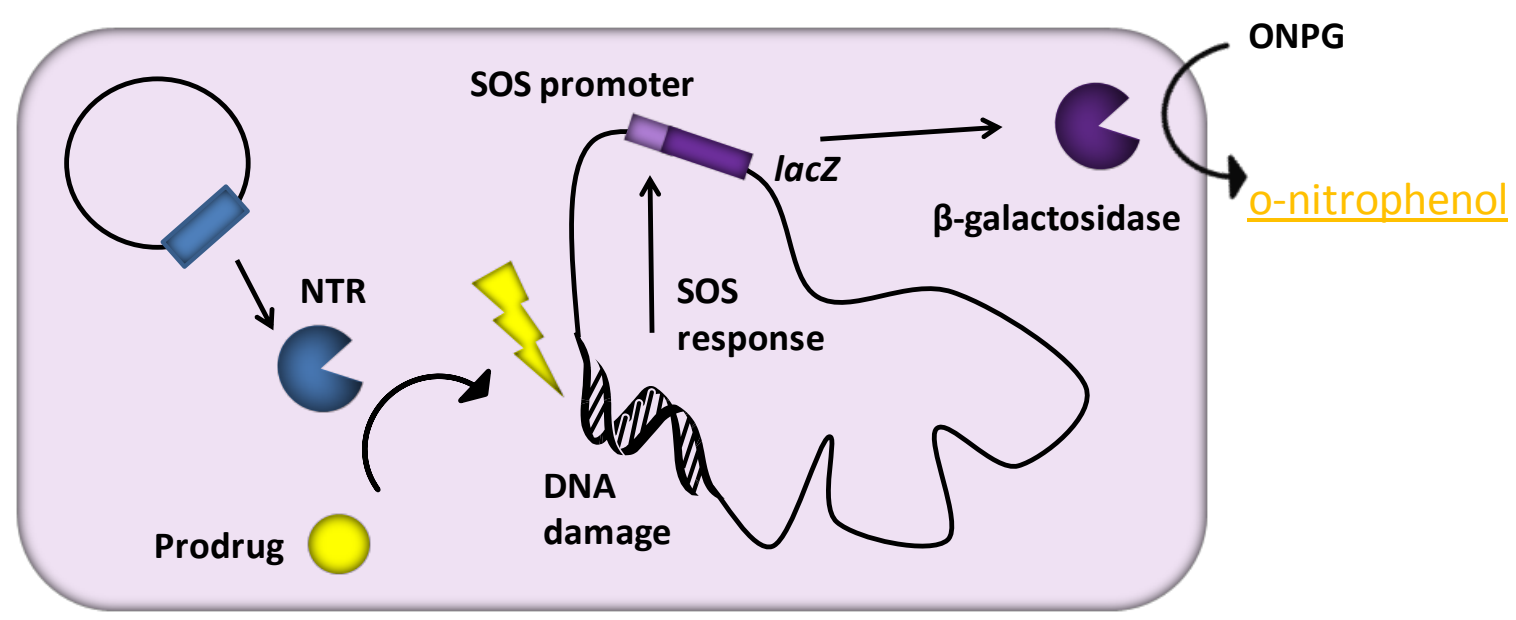

Figure 3-1 Schematic of the SOS assay used to monitor NTR activity with nitroaromatic prodrugs. In specialised E. coli SOS reporter strains the relative abilities of different NTRs to activate DNA damaging nitroaromatic prodrugs are quantitatively determined by measuring the levels of a $\beta$-galactosidase reporter protein, which is induced in the presence of $E$. coli DNA damage (Shapiro and Baneyx, 2002).

In Figure 3-2 can be seen an example of the use of this assay to detect nitroaromatic prodrug conversion, wherein Dr Gareth Prosser used it to interrogate the activity of the 11 E. coli NTR candidates with the prototypical nitroaromatic prodrug CB1954 (Prosser et al., 2010). 


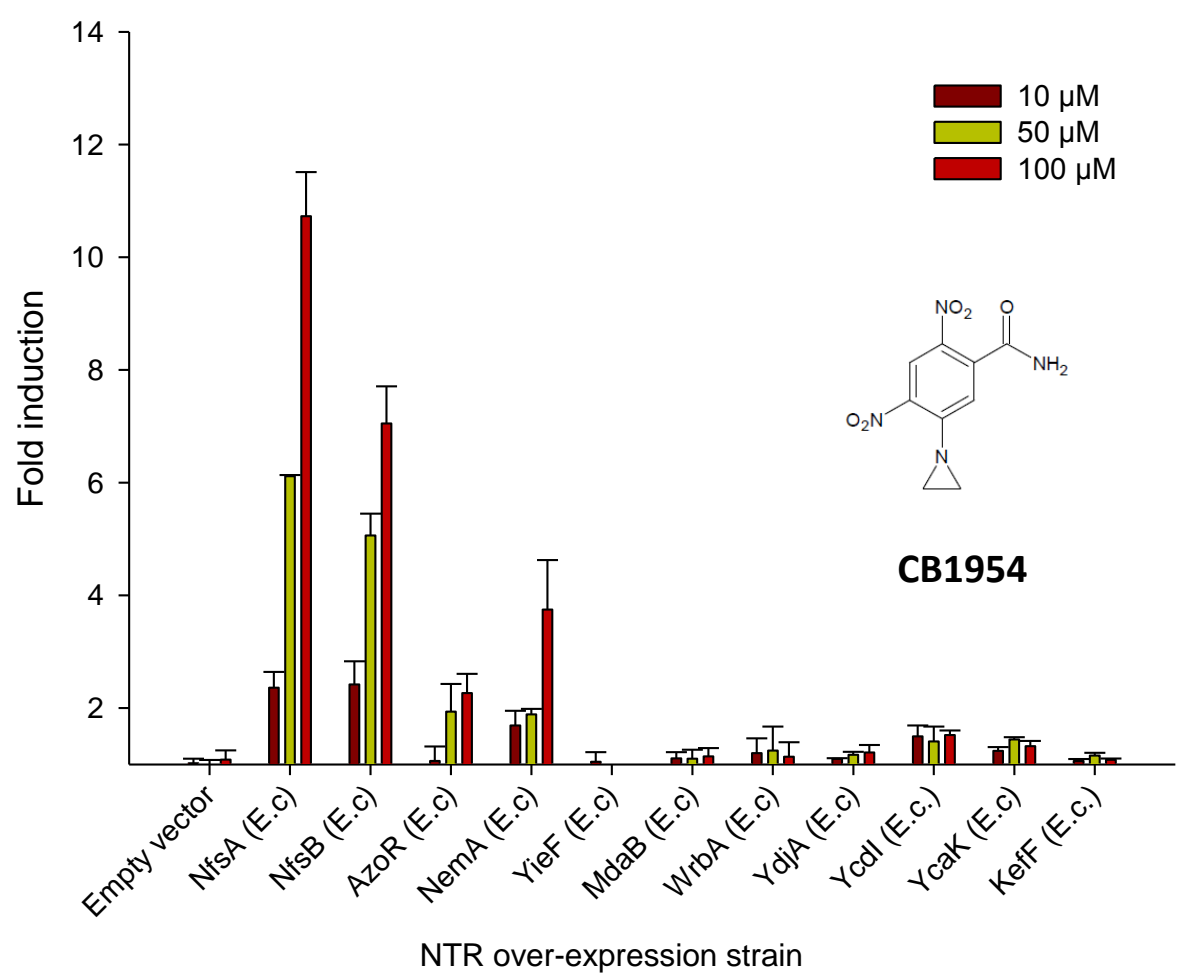

Figure 3-2 CB1954-induced SOS response induction of NTR over-expressing SOS-R1 strainsSOS-R1 strains over-expressing candidate NTRs were grown in liquid media and challenged with the indicated concentrations of CB1954 for $3 \mathrm{~h}$ prior to assessment of SOS response induction. Data are the average \pm 1 SEM of 4 independent assays. Reproduced from (Prosser, 2011).

\subsubsection{Nitroaromatic compounds designed for imaging tumour hypoxia}

The four 2-NI hypoxia imaging agents that were available to this study when work presented in this chapter was carried out are shown in Figure 3-3. All experimental work was performed using these "cold" versions of each imaging agent, which have normal F atoms in the place of where the PET imaging agent would have the radioisotope ${ }^{18} \mathrm{~F}$. Pimonidazole differs from the other compounds presented here in that it cannot be used for noninvasive imaging. It can however be used for biopsy based detection of in vivo hypoxia. This is because there are commercially available fluorescently conjugated monoclonal antibodies raised towards the compound, meaning that patients can be given pimonidazole, a biopsy taken, tumour sections prepared and the in vivo hypoxia dependent pimonidazole binding detected and quantified by immunostaining with the monoclonal antibody (Janssen et al., 2004). Monoclonal antibodies are also available and well validated for biopsy based detection of hypoxia dependent EF5 (Lord et al., 1993) and EF3 (Busch et al., 2000) binding, but not for F-miso. Throughout this thesis screening 
of NTR activity has focused more heavily on EF5 than the prototypical F-miso, as our ACSRC collaborators' preclinical models can detect EF5 binding using traditional immunohistochemistry rather than requiring radiobiological compounds, equipment or expertise.<smiles>O=C(Cn1ccnc1[N+](=O)[O-])NCC(F)(F)F</smiles>

EF5<smiles>O=C(Cn1ccnc1[N+](=O)[O-])NCCC(F)(F)F</smiles>

EF3<smiles>O=[N+]([O-])c1nccn1CC(O)CF</smiles>

F-misonidazole<smiles>O=[N+]([O-])c1nccn1CC(O)CN1CCCCC1</smiles>

Pimonidazole

Figure 3-3 Chemical structures of 2-NI hypoxia imaging agents available to this study

\subsection{Aims}

- Interrogate the ability of $11 \mathrm{E}$. coli NTR candidates to activate 2-NI hypoxia imaging agents

- Optimise microplate based screening methodologies for evaluation of NTR activity with these compounds

- Validate the use of 2-NI's to detect bacterial NTR over-expression by human cancer cells (in this chapter human cell validation was performed by Dr Sophie Syddall and Alex Mowday at the ACSRC)

\subsection{Results}

\subsubsection{Evaluation of NTR activity with 2-NI imaging agents}

\subsubsection{SOS assay}

Prior to this work 11 NTR of $E$. coli origin were cloned into the expression vector pUCX (Prosser et al., 2010) and these constructs were then transformed into the E. coli tester strain SOS-R2. The precursor of this strain, ADA510 (Shapiro and Baneyx, 2002), was kindly supplied by Dr Francois Baneyx and acts as an SOS reporter due to a genomic fusion of the promoter for the SOS inducible gene sfiA with a lacZ reporter gene. In a collaborative laboratory effort we further modified this strain by knocking out five genes using the recombination based protocol of Datsenko and Wanner (Datsenko and Wanner, 
2000) described in further detail in section 2.6. Four of these genes were the E. coli NTRs $n f_{s} A, n f s B, n e m A$ and $a z o R$, previously identified as able to reduce CB1954 (Prosser et al., 2010) and hence removed to lower the endogenous ability of the tester strain to activate nitroaromatic substrates. The fifth gene, tolC encodes an outer membrane protein which is an integral component of the E. coli efflux pump AcrABTolC (Fralick, 1996). A tolC knockout is known to improve sensitivity of E. coli SOS tester strains, particularly with more hydrophobic drugs (Chatterjee and Sternberg, 1995; Shapiro and Baneyx, 2002). It should be noted that, within this chapter, all assays used to screen for CB1954 activation were carried out using our initial tester strain SOS-R1, which contained only the $n f s A$, and $n f s B$ gene deletions.

The SOS response induced in NTR over-expression cultures on exposure to titrated concentrations of the four 2-NI imaging agents were determined as described in detail in section 2.14.1.1. The optical densities $\left(\mathrm{OD}_{600}\right)$ of the cultures were also monitored to detect any growth inhibition. Shown in Figure 3-4A are results with the compound EF5, representative of those seen with all four imaging agents. It can be seen that EF5 does not trigger an SOS response of sufficient magnitude to allow accurate screening using this technique. In the NfsA over-expression culture EF5 does at $200 \mu \mathrm{M}$ elicit a small SOS response, however this is not clearly different than that of the empty control and at this concentration the growth of the NfsA culture has been substantially inhibited (Figure 3-4B). This is in marked contrast to the clear SOS response induced by sub-lethal concentrations of CB1954. Typically when a nitroaromatic compound is reduced in 2eincrements it becomes intrinsically more toxic (Roldán et al., 2008). This is because the increased reactivity of the resulting nitroso and hydroxylamine groups allows binding of the compound to biomacromolecules (i.e. proteins (Leitsch et al., 2007), DNA (Whitmore et al., 1986) and glutathione (Brezden et al., 1997)), which perturbs normal cellular function. That EF5 caused growth inhibition, but only on over-expression of NfsA, did provide preliminary evidence that NfsA could reduce the imaging agent. 


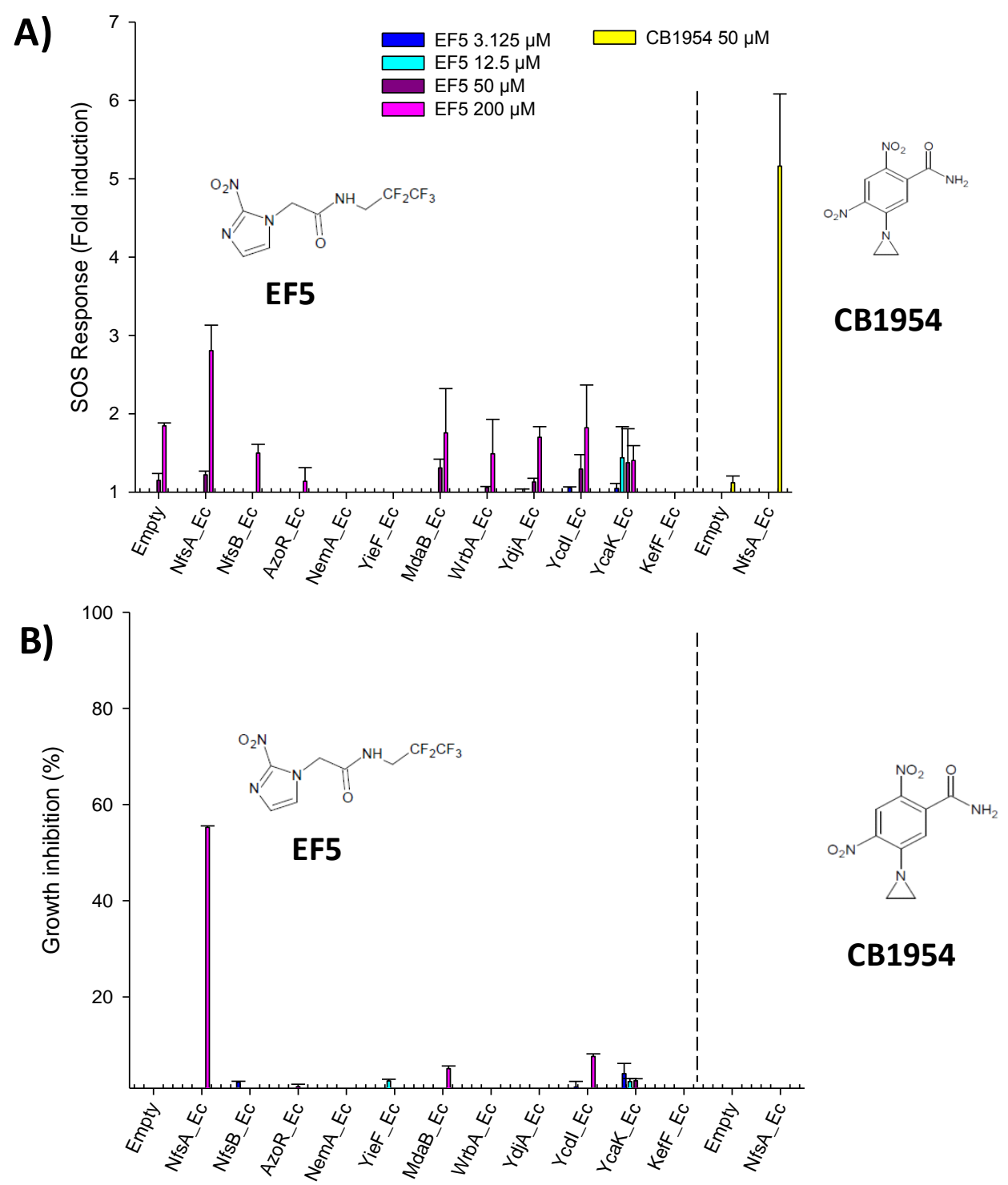

Figure 3-4 E. coli NTR over-expression library exposed to titrated concentrations of EF5 NTR over-expression strains were grown in 96 well microplates and challenged with indicated concentrations of EF5 or CB1954 for 4 hours A) Quantification of SOS response induction by $\beta$ galactosidase assay. Fold induction is derived from the Miller units recorded on drug exposure divided by those of unchallenged control wells B) Growth was measured as culture turbidity $\left(\mathrm{OD}_{600}\right)$ pre and post drug challenge. Growth inhibition is presented as the percentage decrease in the growth of challenged cultures relative to the unchallenged controls for each strain. Data presented here are from one experiment $\pm \mathrm{SD}$, and are representative of many similar experiments.

When initial testing of 2-NI imaging agents revealed that their reduced toxic forms do not induce an SOS response, a fifth compound was added to the testing panel. This was the 2NI drug RB6145, a drug which in physiological conditions shows a $\sim 30 \%$ conversion to a 
second compound RSU1069 (Bremner, 1993; Jenkins et al., 1990), both compounds are shown in Figure 3-5. RSU1069 is not an imaging agent but rather was designed as a dual function hypoxic cell radiosensitizer and hypoxia activated chemotherapeutic.

However severe dose limiting gastric toxicity in Phase I trials stymied its clinical development and spurred interest in analogues such as RB6145 (Jenkins et al., 1990). Similar to CB1954, the presence of the DNA alkylating aziridine ring means nitro reduction converts RSU1069 from a weak monofunctional alkylating agent to a potent DNA cross linker (Bremner, 1993).<smiles>O=[N+]([O-])c1nccn1CC(O)CNCCBr</smiles>

RB6145<smiles>O=[N+]([O-])c1nccn1CC(O)CN1CC1</smiles>

RSU1069

Figure 3-5 Chemical structures of the 2-NI RB6145 (used in this study) and the compound RSU1069 to which RB6145 shows $\sim 30 \%$ conversion to in physiological conditions (Jenkins et al., 1990).

Based on this, we hypothesised that reduction of RSU1069 or RB6145 would be detectable by the SOS assay, and this hypothesis was found to be true (Figure 3-6). As with the 2-NI imaging agents, only NfsA showed significant activity with RB6145, with a $2 \mu \mathrm{M}$ challenge concentration sufficient to generate a near 10 -fold increase in SOS induction (a response near saturation as evidenced by a doubling of RB6145 concentration failing to significantly increase the level of SOS induction). While other NTR candidates did induce a slight SOS response upon RB6145 challenge this was likely due to intrinsic genotoxicity of the parental molecule, with levels of SOS induction not significantly greater than those seen with the empty plasmid control. 


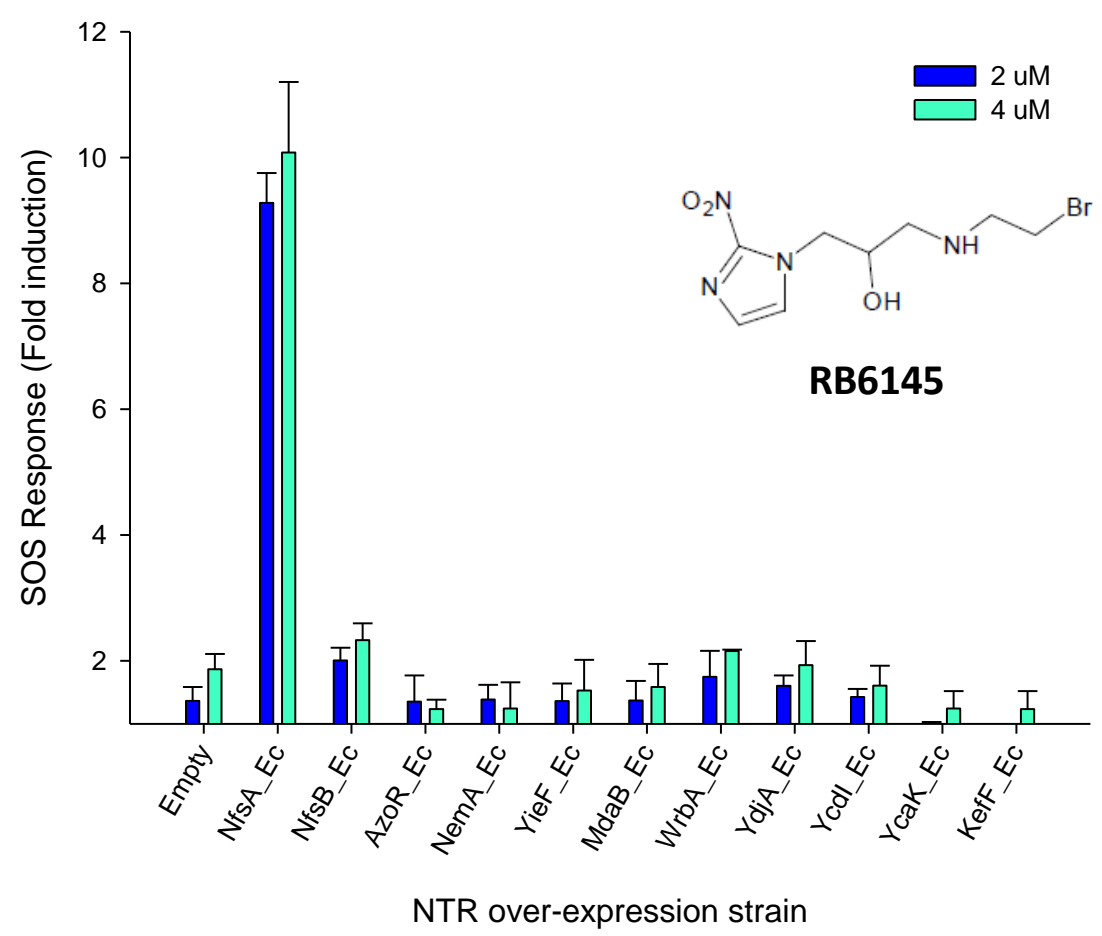

Figure 3-6 RB6145 induced SOS response of the $E$. coli NTR over-expression library SOSR2 NTR over-expression strains were grown in 96 well microplates and challenged with indicated concentrations of RB6145 for 4 hours prior to quantification of SOS response induction by $\beta$ galactosidase assay. Fold induction is derived from the Miller units recorded on drug exposure divided by those of unchallenged control wells. Data are the mean of two independent experiments \pm SEM.

\subsubsection{Growth inhibition}

To confirm the apparent ability of NfsA to sensitize E. coli to 2-NI compounds, IC 50 values were determined (Figure 3-7). The $\mathrm{IC}_{50}$ value is the drug concentration required to reduce strain growth to $50 \%$ of that of unchallenged controls, here determined by monitoring the culture density $\left(\mathrm{OD}_{600^{-}}\right.$the cultures light absorbance at $\left.600 \mathrm{~nm}\right)$ of overexpression strains exposed to titrated drug concentrations for four hours (described in section 2.14.3).

The only NTRs which showed detectable activity with any of the compounds were NfsA and NfsB. NfsA sensitised E. coli to all five of the 2-NI compounds at readily acheivable drug concentrations. The NfsB over-expression strain showed low-level activity with both $\mathrm{EF} 3$ and F-miso, with the $\mathrm{IC}_{50}$ of these compounds was $\sim 7.5$ to $\sim 8$ fold higher than that seen on NfsA over-expression. 


\begin{tabular}{cccccc}
\hline \multicolumn{7}{c}{ IC $_{50}(\mu \mathrm{M})$} \\
\hline Empty & $>1000$ & $>1000$ & $>1000$ & $>1000$ & $>250$ \\
NfsA (E.c) & $141 \pm 9$ & $83 \pm 4$ & $112 \pm 1$ & $175 \pm 5$ & $14 \pm 1$ \\
NfsB (E.c) & $>1000$ & $685 \pm 78$ & $859 \pm 22$ & $>1000$ & $>250$ \\
AzoR (E.c) & $>1000$ & $>1000$ & $>1000$ & $>1000$ & $>250$ \\
NemA (E.c & $>1000$ & $>1000$ & $>1000$ & $>1000$ & $>250$ \\
YieF (E.c) & $>1000$ & $>1000$ & $>1000$ & $>1000$ & $>250$ \\
MdaB (E.c) & $>1000$ & $>1000$ & $>1000$ & $>1000$ & $>250$ \\
WrbA (E.c) & $>1000$ & $>1000$ & $>1000$ & $>1000$ & $>250$ \\
YdjA (E.c) & $>1000$ & $>1000$ & $>1000$ & $>1000$ & $>250$ \\
YcdI (E.c.) & $>1000$ & $>1000$ & $>1000$ & $>1000$ & $>250$ \\
YcaK (E.c) & $>1000$ & $>1000$ & $>1000$ & $>1000$ & $>250$ \\
KefF (E.c.) & $>1000$ & $>1000$ & $>1000$ & $>1000$ & $>250$ \\
\hline
\end{tabular}

Figure 3-7 $\mathrm{IC}_{50 \mathrm{~s}}$ of 2-NI compounds for the SOS-R2 E. coli NTR over-expression library Drug dependent growth inhibition was monitored by measuring strain turbidity $\left(\mathrm{OD}_{600}\right)$ pre and post four hour incubation with a two-fold dilution series of respective drugs, with a top concentration of $1 \mathrm{mM}$ chosen to conserve imaging agent stocks. Percentage growth relative to unchallenged controls was determined and used to calculate the concentration at which $50 \%$ growth inhibition was seen using SigmaPlot ${ }^{\mathrm{TM}}$ 10.0. Data are the average of at least two independent assays \pm SEM.

\subsubsection{Purified protein kinetics}

To support the in vivo observations and also to confirm that each of the 2-NI compounds was reduced directly by candidate nitroreductases (as opposed to via other intermediate substrates) each of the enzymes were purified and their ability to reduce these 2-NI compounds was quantified in vitro. All 11 E. coli NTR candidates had been previously cloned into the plasmid pET-28a(+), which allows for the expression of N-terminal His6 tagged proteins that can be purified by nickel affinity chromatography (section 2.10 ). The pET:ntr constructs were each transformed into the E. coli strain BL:21, and NTRs were over-expressed by overnight growth of the strains in autoinduction media at $30{ }^{\circ} \mathrm{C}$. Consistent with previous observations (Prosser, 2011) only nine of the 11 E. coli NTRs were able to be purified, with YcdI and KefF being found predominantly in the insoluble fraction (results not shown). Strategies to improve their soluble over-expression were not pursued due to their lack of in vivo activity with 2-NI compounds. 
For the successfully purified NTR candidates, reaction rates were determined using spectrophotometry, as both the oxidation of $\mathrm{NAD}(\mathrm{P}) \mathrm{H}$ and the reduction of the 2-NI compound causes a decrease in light absorbance at $340 \mathrm{~nm}$ which is directly proportional to the quantity of compound metabolised by the NTR (section 2.11.1). This allowed measurement of the intial reaction rate when purified enzyme, a fixed concentration of $\mathrm{NADPH}$, and varying concentrations of 2-NI compound were incubated together.

\begin{tabular}{|c|c|c|c|c|}
\hline NTR & Compound & $K_{m}^{a}(\mu \mathrm{M})$ & $\begin{array}{l}k_{\text {cat }}^{a} \\
\left(s^{-1}\right)\end{array}$ & $\begin{array}{c}k_{\text {cat }} / K_{m} \\
\left(\mathrm{mM}^{-1} \mathrm{~s}^{-1}\right)\end{array}$ \\
\hline \multirow[t]{5}{*}{ NfsA (E.c) } & EF5 & $160 \pm 10$ & $12.1 \pm 0.2$ & 76 \\
\hline & EF3 & $160 \pm 21$ & $10.7 \pm 0.4$ & 66 \\
\hline & F-miso & $480 \pm 70$ & $8.8 \pm 0.6$ & 18 \\
\hline & Pimo & $240 \pm 26$ & $11.3 \pm 0.5$ & 47 \\
\hline & RB6145 & $200 \pm 33$ & $2.7 \pm 0.2$ & 14 \\
\hline \multirow[t]{4}{*}{ NfsB (E.c) } & EF5 & $1800 \pm 840$ & $0.4 \pm 0.1$ & 0.2 \\
\hline & EF3 & $5800 \pm 3700$ & $1.1 \pm 0.5$ & 0.2 \\
\hline & F-miso & $5200 \pm 3100$ & $2.1 \pm 1.0$ & 0.4 \\
\hline & Pimo, RB6145 & \multicolumn{3}{|c|}{ No detectable activity } \\
\hline AzoR (E.c) & EF5, EF3, F-miso, Pimo, RB6145 & \multicolumn{3}{|c|}{ No detectable activity } \\
\hline NemA (E.c) & EF5, EF3, F-miso, Pimo, RB6145 & \multicolumn{3}{|c|}{ No detectable activity } \\
\hline YieF (E.c) & EF5, EF3, F-miso, Pimo, RB6145 & \multicolumn{3}{|c|}{ No detectable activity } \\
\hline MdaB (E.c) & EF5, EF3, F-miso, Pimo, RB6145 & \multicolumn{3}{|c|}{ No detectable activity } \\
\hline WrbA (E.c) & EF5, EF3, F-miso, Pimo, RB6145 & \multicolumn{3}{|c|}{ No detectable activity } \\
\hline YdjA (E.c) & EF5, EF3, F-miso, Pimo, RB6145 & \multicolumn{3}{|c|}{ No detectable activity } \\
\hline YcaK (E.c) & EF5, EF3, F-miso, Pimo, RB6145 & \multicolumn{3}{|c|}{ No detectable activity } \\
\hline
\end{tabular}

Table 3-1 Kinetic parameters for the reduction of 2-NI compounds by purified His6-tagged NTRs Rates of reduction at varying 2-NI concentrations and a fixed concentration of excess NADPH $(0.20 \mathrm{mM})$ were monitored at $340 \mathrm{~nm}$, rates measured in quadruplicate. Apparent $K_{m}$ and $k_{\text {cat }}$ derived using Sigmaplot 10.0 (Systat Software Inc., Richmond, CA). Extinction coefficients used were EF5 $=19,000 \mathrm{M}^{-1} \mathrm{~cm}^{-1}, \mathrm{EF} 3=19,100 \mathrm{M}^{-1} \mathrm{~cm}^{-1}$, F-miso $=18,900 \mathrm{M}^{-1} \mathrm{~cm}^{-1}$, Pimo $=$ $18,600 \mathrm{M}^{-1} \mathrm{~cm}^{-1}, \mathrm{RB} 6145=19,500 \mathrm{M}^{-1} \mathrm{~cm}^{-1}$ for explanation of determination see section 2.10. Due to the lack of co-factor preference exhibited by NfsB and the strong NADPH preference of NfsA (Bryant et al., 1981) only apparent kinetic parameters with NADPH as cofactor were determined.

As per the in vivo results only $\mathrm{NfsA}$ and $\mathrm{NfsB}$ showed any activity with the 2-NI compounds, with NfsA substantially more active than NfsB. The highest concentration of drug able to be measured was $1200 \mu \mathrm{M}$, as above this the spectrophotometer did not register a linear relationship between light absorbance at $340 \mathrm{~nm}$ and compound 
concentration. For this reason the apparent kinetic values for NfsB show a high degree of error. Nonetheless, while this error should be noted, the difference between NfsA and $\mathrm{NfsB}$ activity is substantial. In regards to EF5, NfsA was 400 fold more active (in terms of the specificity constant $\left.k_{c a t} / K_{m}\right)$ and even in the case of F-miso, the compound with which NfsB showed the greatest activity, the specificity constant of NfsB was still 44 fold lower than that of NfsA.

\subsubsection{Optimisation of a microplate based $\mathrm{NAD}(\mathrm{P}) \mathrm{H}$ depletion assay}

Our inability to use the SOS assay to determine NTR activity with each of the 2-NI imaging agents led us to pursue other screening options. The requirement for $\mathrm{NAD}(\mathrm{P}) \mathrm{H}$ as an electron source for NTR activity pointed to the possibility of using an in vivo chemical assay which reported on $\mathrm{NAD}(\mathrm{P}) \mathrm{H}$ depletion. A major advantage of such an approach is that it would allow for screening of NTR activity irrespective of substrate.

While direct measurement of $\mathrm{NAD}(\mathrm{P}) \mathrm{H}$ usage is possible via monitoring $340 \mathrm{~nm}$ absorbance, doing so in a reproducible and high-throughput microplate screen is complicated by the relatively low signal and the background absorbance at this wavelength from both the microplate and crude lysates (Mayer and Arnold, 2002). One alternative is to use tetrazolium salts to indirectly report on $\mathrm{NAD}(\mathrm{P}) \mathrm{H}$ levels as, in the presence of a phenazine methosulfate (PMS) catalyst, NAD(P)H chemically converts a number of tetrazolium salts into formazan products with a concomitant large change in absorbance spectra (Luo et al., 2006).

Essentially a crude lysate of the NTR over-expression culture, the substrate in question and $\mathrm{NAD}(\mathrm{P}) \mathrm{H}$ are combined and left for a set period of time, with $\mathrm{NAD}(\mathrm{P}) \mathrm{H}$ being depleted if an oxidoreductase reaction occurs. The reaction is then be halted by addition of nitroblue tetrazolium chloride (NBT)/PMS and any remaining $\mathrm{NAD}(\mathrm{P}) \mathrm{H}$ then chemically reduces NBT to a formazan dye, the production of which is measured by an absorbance reading at $\mathrm{OD}_{590}$ and directly correlates to concentration of NADPH that was remaining in the reaction at that time (Glieder and Meinhold, 2003). A schematic is shown in Figure 3-8. 
A) 1. Reaction initiated on $\mathrm{NAD}(\mathrm{P}) \mathrm{H}$ addition

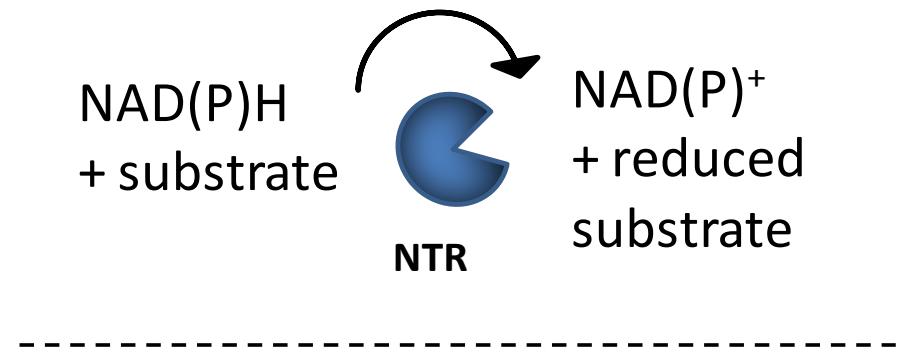

2. Reaction halted and residual $\mathrm{NAD}(\mathrm{P}) \mathrm{H}$ measured via NBT/PMS addition
B)

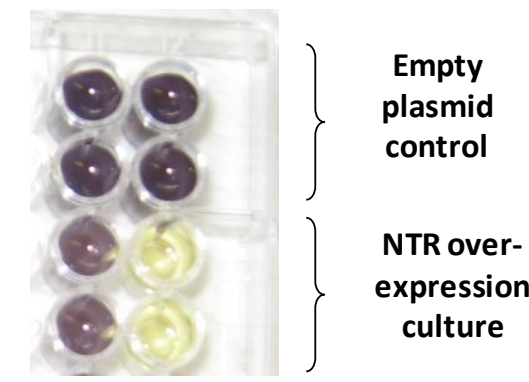

Substrate added
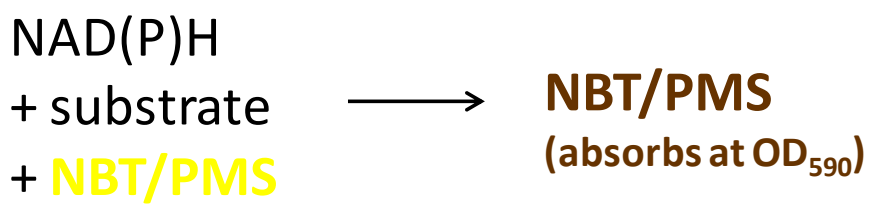

Figure 3-8 NAD(P)H depletion assay A) Schematic showing use of NBT/PMS to determine remaining $\mathrm{NAD}(\mathrm{P}) \mathrm{H}$ following an NTR catalysed oxidoreductase reaction $\mathbf{B})$ Photo of application in 96 well microplate, taken on measuring reduction of the prodrug substrate PR-104A by a NfsA over-expression culture.

\subsubsection{Optimisation of assay parameters}

Before the NAD $(\mathrm{P}) \mathrm{H}$ depletion assay was used to screen for NTR activity with 2-NI compounds a number of reaction parameters were optimised (results not shown). These included the concentrations of NBT and $\mathrm{NAD}(\mathrm{P}) \mathrm{H}$ used; development of a method for reproducibly achieving complete and even cell lysis of the E. coli cultures; identification of the substrate incubation periods and cell lysate volumes best suited for detection of NTR activity (section 2.14.4). It was also demonstrated that the possibility of NTR catalysed reduction of the nitro-group present in NBT did not interfere with the assay (results not shown).

\subsubsection{CB1954 reduction- SOS assay vs. NAD(P) H depletion assay}

To validate the $\mathrm{NAD}(\mathrm{P}) \mathrm{H}$ depletion assay it was used to measure the activity of the $E$. coli NTRs with the prodrug CB1954 and these results were compared to those of a SOS assay of the same enzymes as determined by Dr. Gareth Prosser (previously depicted in Figure 3-2). Separate assays were carried out using both NADPH and NADH cofactors. Initially the reactions were allowed to proceed for only 20 minutes and during this time only NfsA, NfsB and NemA showed significant activity. However allowing subsequent reactions to run for 80 minutes demonstrated that AzoR EC also utilized both NADH and 
NADPH in the presence of CB1954. NfsB was also equally efficient with both cofactors, whereas NfsA and NemA showed a preference for NADPH. As such both the NAD(P)H depletion assay and the SOS assay identified the same NTRs as able to reduced CB1954 and ranked their activities (NfsA $>\mathrm{NfsB}>\mathrm{NemA}>\mathrm{AzoR}$ ) in an identical manner.
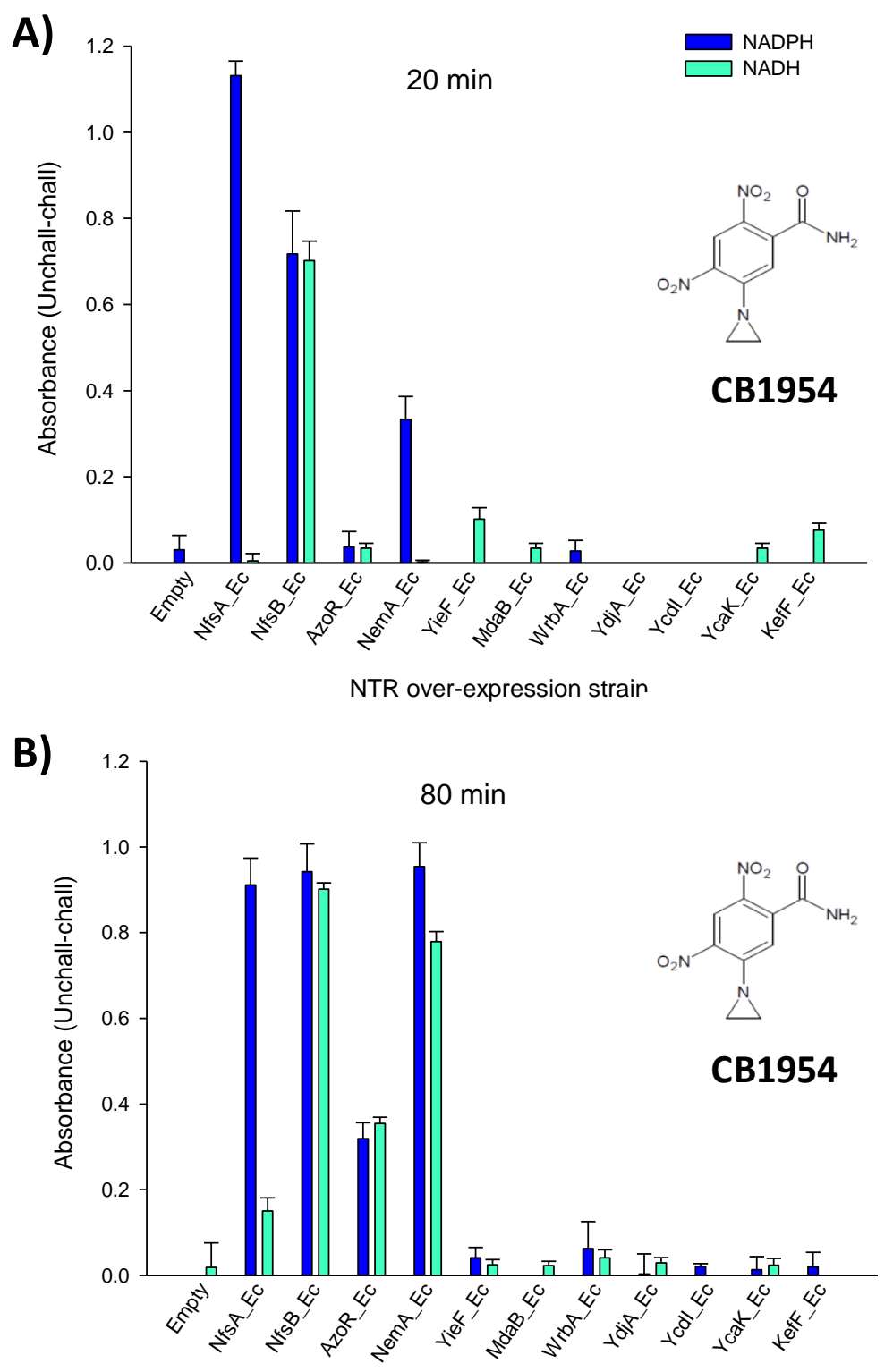

NTR over-expression strain

Figure 3-9 NAD(P)H consumption by SOS-R1:ntr cell lysates in the presence of CB1954. Crude cell lysates were incubated with equimolar concentrations of both CB1954 and either NADH or NADPH for (A) 20 min or (B) 80 min. Addition of NBT post-incubation yielded formazan dye in proportion remaining $\mathrm{NAD}(\mathrm{P}) \mathrm{H}$, which was quantified by measuring absorbance at OD590. Plotted values indicate the extent of CB1954 metabolism by each NTR over-expressing strain and were derived by subtracting the OD590 of CB1954-challenged lysates (chall) from the OD590 of unchallenged duplicate controls (Unchall). Data are the average of three independent assays \pm SEM. 


\subsubsection{Evaluation of NTR activity with 2-NI imaging agents using the NAD(P)H depletion assay}

Following this validation the NAD(P)H depletion assay was used to assess activity of the E. coli NTRs with each of the five 2-NI compounds (Figure 3-10A). The assay was left for 30 mins to allow potential detection of low levels of NTR activity (however NfsA consumed all supplied NADPH within $\sim 5$ mins of exposure to EF5 (Figure 3-10B). Not surprisingly only NfsA expressing cell lysates showed substantial depletion of NADPH in the presence of any of the 2-NI compounds. When NADH was used as the cofactor no NTR activity was detected, emphasising the strong cofactor preference of NfsA (results not shown). The assay proved somewhat less sensitive than purified protein kinetics as it could not detect the very low levels of activity NfsB showed with EF5, EF3 and F-miso, irrespective of the conditions employed.

It should be noted that to minimize the amount of (valuable and supply-limited) imaging agent consumed, only $150 \mu \mathrm{M}$ of each substrate was incubated with $200 \mu \mathrm{M}$ NADPH. As NTRs reduce nitroaromatic compounds to hydroxylamine derivatives in two consecutive 2e- steps, they oxidise two NAD(P)H molecules for every molecule of compound reduced and when $150 \mu \mathrm{M}$ of 2 -NI substrate was used reaction rates were not substantially affected (results not shown). 
A)

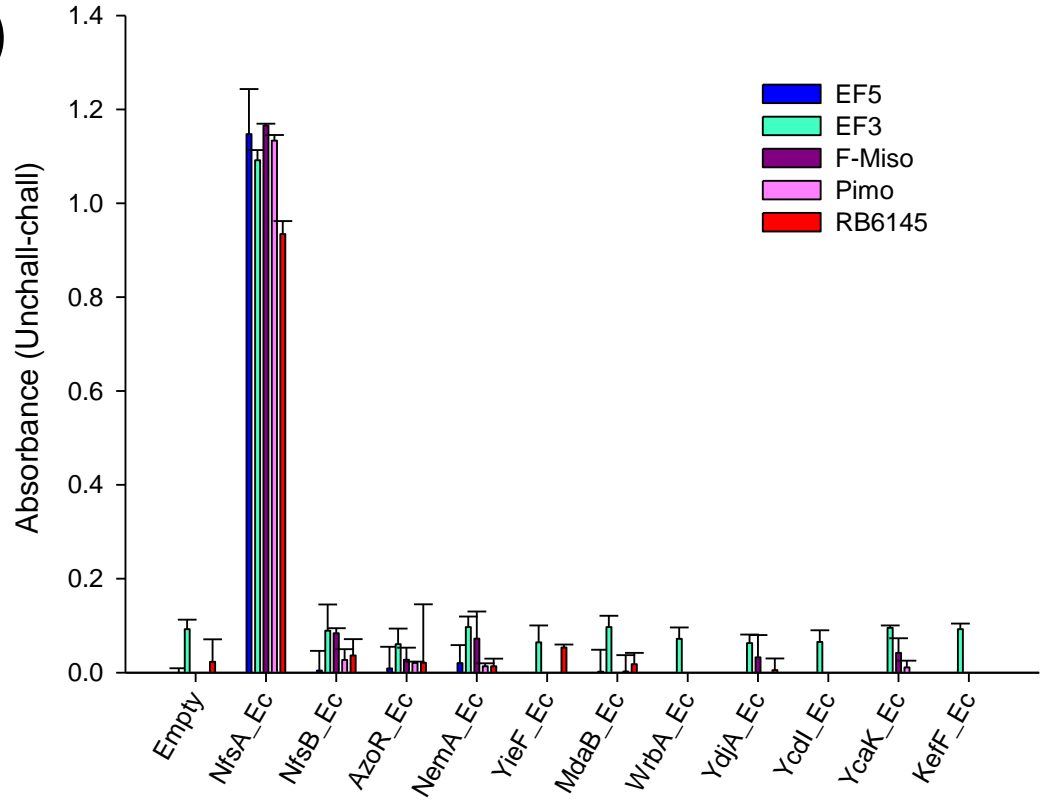

B) NTR over-expression strain

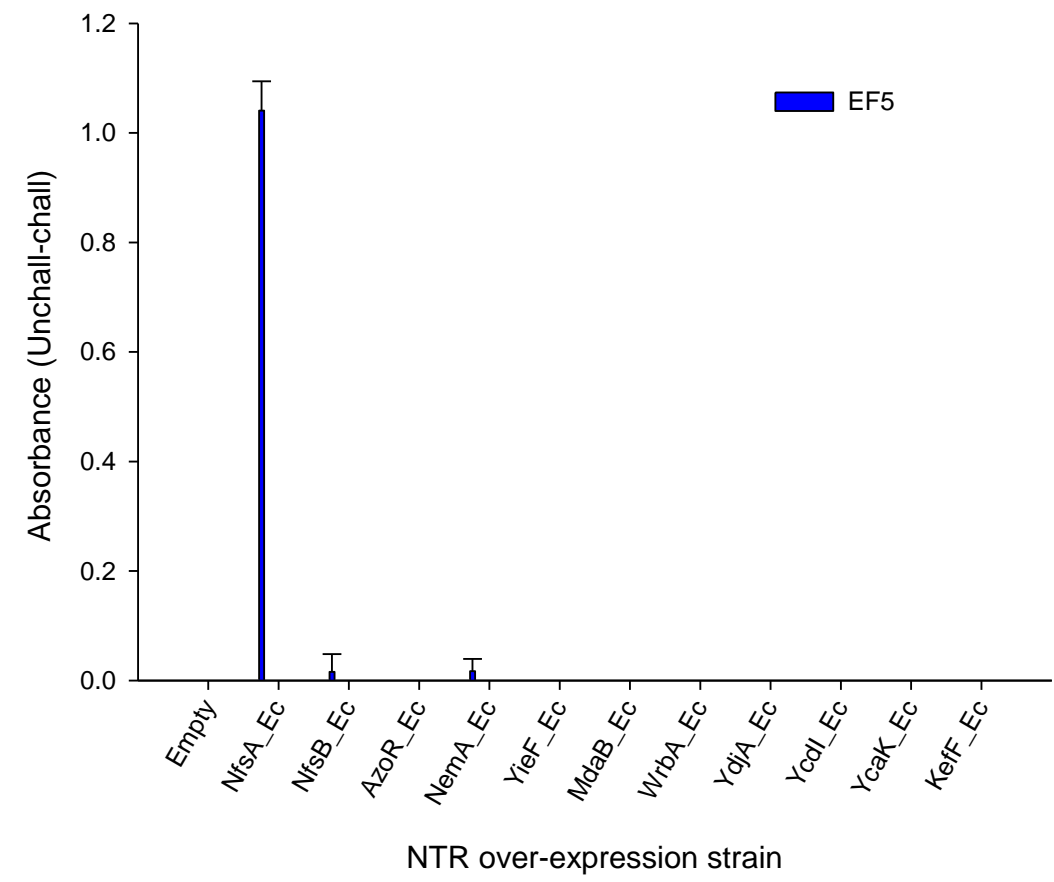

Figure 3-10 NADPH consumption by SOS-R2:ntr cell lysates in the presence of 2-NI compounds. Crude cell lysates were incubated with $200 \mu \mathrm{M}$ NADPH and $150 \mu \mathrm{M}$ stated compound for A) 30 mins B) 5 mins. Addition of NBT post-incubation yielded formazan dye in proportion to the remaining $\mathrm{NAD}(\mathrm{P}) \mathrm{H}$, which was quantified by measuring absorbance at $\mathrm{OD}_{590}$. Plotted values indicate the extent of 2-NI metabolism by each NTR over-expressing strain and were derived by subtracting the $\mathrm{OD}_{590}$ of compound challenged lysates (chall) from the $\mathrm{OD}_{590}$ of unchallenged duplicate controls (Unchall). Data are the average of two independent assays \pm SEM. 


\subsection{Discussion}

The ability to noninvasively image transgenic NTR expression level and localisation would be extremely advantageous to preclinical and clinical development of NTR cancer gene therapy. Successful demonstration of this technology could also position NTRs to be used as reporter enzymes for imaging of GDEPT-independent oncolytic viruses and bacteria. Development of this capability would be facilitated if we could repurpose imaging agents that have already undergone independent Phase I validation, namely the 2-nitroimidazole hypoxia imaging agents.

As part of a collaborative effort to develop NTR based GDEPT our laboratory generated a small E. coli based gene over-expression library of 11 diverse candidate NTRs. Exposure of $E$. coli over-expressing these NTRs to four 2-NI imaging agents demonstrated that one enzyme, NfsA, the "major" E. coli NTR could metabolise these agents; whereas the prototypical NTR used in GDEPT, NfsB, showed low or no detectable activity with the compounds. This reduction was determined to be a result of direct enzyme activity by spectrophotometric monitoring of the reaction as carried out by purified proteins.

E. coli based screening for NTR-catalysed 2-NI metabolism using the SOS assay, which reports on DNA damage, was successful only for detecting NTR activation of the nitromasked DNA cross-linking agent RB6145. However as the reduced metabolites of 2-NIs were found to be directly toxic to E. coli, 2-NI reduction can at least be detected by monitoring the 2-NI dependent growth inhibition of different E. coli NTR overexpression strains. In pursuit of other screening options I investigated the use of a microplate based colorimetric $\mathrm{NAD}(\mathrm{P}) \mathrm{H}$ depletion assay. This assay was shown to be able to detect NTR reduction of both the nitroaromatic prodrug CB1954 and of 2-NI imaging agents. In addition to supporting screening of 2-NIs, such an assay could widely applicable in our laboratory as it could be used to monitor NTR activity with other nitrotriggered prodrugs that would not elicit an SOS response (or any other phenotype in bacteria) for example nitro-triggered protein kinase inhibitors.

To validate this 2-NI metabolism, then PhD student Dr. Sophie Syddall at the ACSRC generated 11 HCT-116 (human colon carcinoma) cell lines stably expressing the different NTRs and assessed the ability of each transfected cell line to metabolise EF5. EF5 binding (as determined by fluorescent microscopy and flow cytometry of HCT-116:ntr cells exposed first to EF5 and then the fluorescent anti-EF5 antibody) was found, in oxic 
conditions, to occur only on NfsA expression (Syddall, 2009). This demonstrated that a bacterial NTR could metabolise a 2-NI imaging agent to its cell entrapped form in human cells, and provided initial validation for our strategy of discovering such NTRs by measuring 2-NI dependent growth inhibition and NADPH consumption in E. coli.

Figure 3-11 shows a later experiment performed by $\mathrm{PhD}$ candidate Alex Mowday at the ACSRC. This is an example of biopsy based detection of EF5 binding in HCT116:NfsA_Ec tumour xenografts from mice, showing that this reduction is substantially more efficient than both background activation in hypoxic regions of tumours and activation in NfsB expressing tumours. Furthermore Alex Mowday showed that when mice carrying mixed HCT-116/HCT-116:NfsA_EC xenografts were treated with both PR-104A and EF5, fluorescence of excised tumours correlated with tumour cell kill, an important result supporting the possible use of 2-NI imaging agents to predict efficacy of NTR based GDEPT (Figure 3-12).

These results demonstrate that we have the beginnings of a useful tool for detecting NTR over-expression in our preclinical models. However while immunohistochemically detected 2-NI activation by stably expressed NfsA is distinguishable from the hypoxic background, this does not guarantee that PET scanning will be able to detect vector mediated NTR expression. The feasibility of this strategy can only be truly determined by carrying out microPET in preclinical cancer models and later PET in patients. As such we have recently developed a collaboration with researchers at Maastricht University in the Netherlands (www.maastrichtuniversity.nl) and experiments to achieve this microPET validation are ongoing.

While we have discovered that NfsA from E. coli can carry out 2-NI metabolism this was of the result of screening only a small enzyme library. Restricting further study to the first NTR found to display this metabolism, without further assessment of what is a very widespread enzyme class, may cause us to overlook NTRs with substantially better activity. It is nonetheless promising that NfsA could metabolise all of the 2-NI substrates regardless of their exact side chain, as this substrate promiscuity suggests that 2-NI metabolism may be a general property of the NfsA NTR subfamily. 
A)
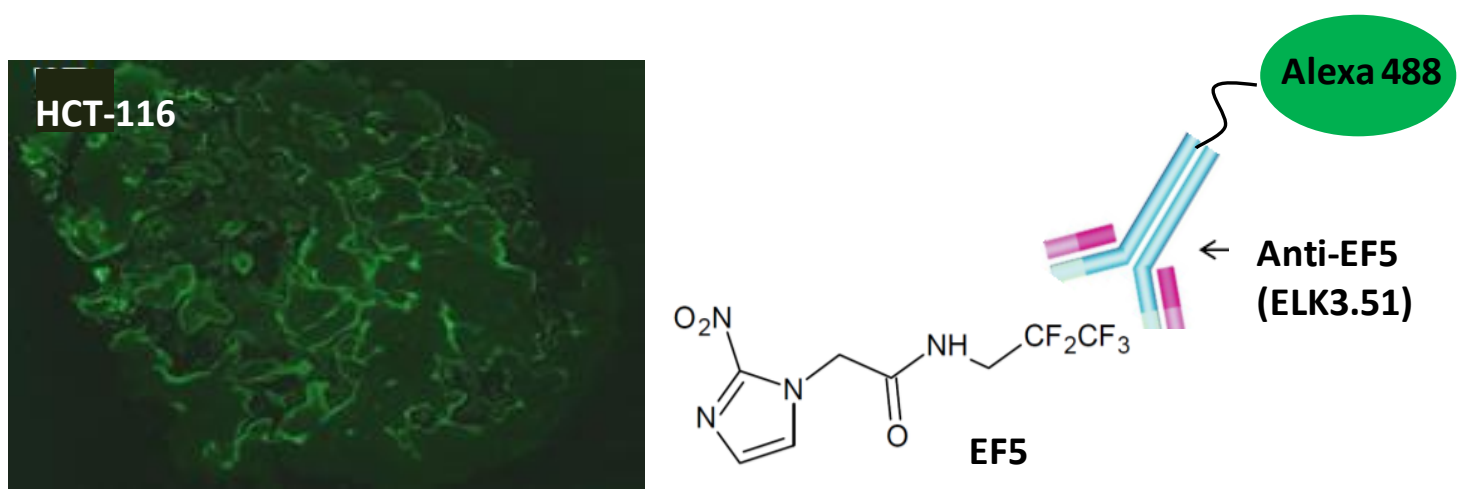

B)
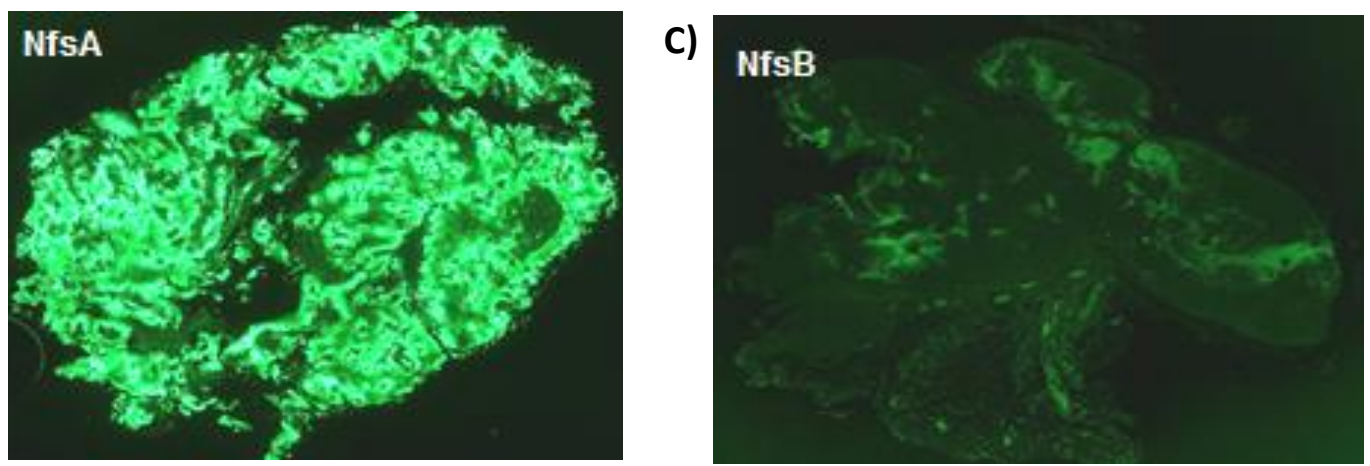

Figure 3-11 In vivo binding of EF5 in HCT-116:ntr tumour xenografts

HCT-116 tumours were established in NIH III nude mice via subcutaneous injection of $10 \times 10^{6}$ cells in $100 \mu \mathrm{L}$ serum free media. Cells added comprised A) $100 \%$ wild type HCT-116 cells B) 25\% HCT-116:NfsA_Ec cells C) 25\% HCT-116:NfsB_Ec cells. Mice were treated with $60 \mathrm{mg} / \mathrm{kg}$ EF5 by intraperitoneal injection and left for three hours. Tumours were then excised and fixed in $10 \%$ formalin for $48 \mathrm{hrs}$, then paraffin embedded, sectioned and mounted on glass slides for immunohistochemical analysis with anti-EF5 antibody Alexa-488 ELK3.51 (100 $\mu \mathrm{g} / \mathrm{ml})$. Sections were scanned on a Nikon 2000E Inverted microscope. Reproduced with permission from Alex Mowday and Adam Patterson (ACSRC). 


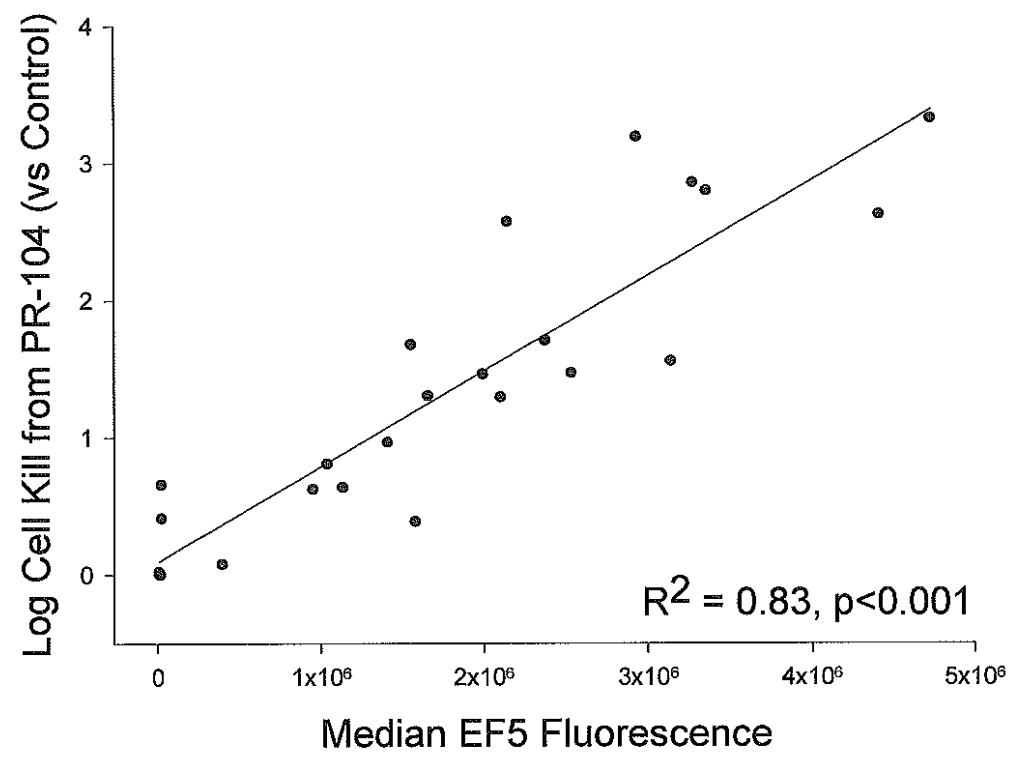

Figure 3-12 Comparison of in vivo PR-104A tumour cell kill and EF5 binding Mixed HCT-116 tumour xenografts were established in NIH III nude mice by subcutaneous injection of $10 \times 10^{6}$ cells comprised of mixtures of HCT-116 and HCT-116:NfsA_Ec cells using fixed ratios of $0 \%, 1 \%, 3 \%, 5 \%, 10 \%, 15 \%$ or $25 \%$ NfsA_Ec expressing cells. Mice bearing these established mixed tumours were treated with PR-104 (325 mg/kg; intraperitoneal injection). Three hours later EF5 (30 mg/kg, intraperitoneal injection) was administered to label cells and after a further two hours tumour tissue was excised and subjected to enzymatic disaggregation to form a single cell suspension. Cells were then processed in two ways; i) plated in triplicate over a range of cell densities $\left(10^{5}-10^{2}\right.$ cells/plate) and surviving colonies were counted on day 10 and used to determine log cell kill; ii) fixed in 4\% paraformaldehyde, labelled with anti-EF5 antibody Alexa-488 ELK3.51 $(100 \mu \mathrm{g} / \mathrm{ml})$ and subjected to flow cytometry using a Becton Dickinson LSRII Flow Cytometer (Becton Dickinson Biosciences, Bedford, MA). Fluorescence was excited using the $355 \mathrm{~nm}$ UV laser and fluorescence emission monitored at $450 \mathrm{~nm} \pm 25$. Reproduced with permission from Alex Mowday and Adam Patterson (ACSRC). 


\section{Chapter 4: Screening of an expanded bacterial oxidoreductase library for enzymes which metabolise 2-nitroimidazole imaging agents}

\subsection{Introduction}

The previous chapter presented work evaluating 11 E. coli NTR candidates for their ability to metabolise previously developed 2-NI PET imaging agents and showed that the enzyme NfsA demonstrated such metabolism. We next sought to interrogate NTR candidates from other bacteria to see if we could uncover NTRs with superior activity to E. coli NfsA.

The previous chapter also presented three different strategies for screening for 2-NI reduction by $E$. coli NTR over-expression strains, i.e. measurement of 2-NI dependent NADPH consumption by cell lysates, 2-NI dependent $E$. coli growth inhibition, and use of the SOS assay to monitor 2-NI induced DNA damage (this last strategy only useful for detecting NTR activity with the analogous 2-NI compound RB6145). In the work described in this chapter, these strategies are applied to screening an expanded NTR candidate library.

\subsubsection{Expanded NTR candidate library}

Bacterial oxidoreductase enzymes are ubiquitous throughout nature and have a number of potential biotechnological applications. As such, numerous researchers in our laboratory have collaborated in the construction of an E. coli over-expression library of 58 NTR candidate enzymes. Activities for which the enzymes in this library have been screened include DNA damaging prodrug activation (Prosser et al., 2013), chromate reduction for bioremediation purposes (Robins et al. 2013), the activation of novel NTR activated fluorogenic probes (Horvat, 2012) and, as presented here, 2-NI imaging agent metabolism.

The library comprises oxidoreductases derived from 20 different bacterial species and 13 different enzyme families; orthologs of the 11 E. coli NTR candidates identified in Chapter 3, and of two additional enzymes previously shown to metabolise CB1954- the rat oxidoreductase NQO1 (Knox et al., 1988) and the enzyme YwrO from $B$. amyloliquefaciens (Anlezark et al., 2002). Based on a range of preliminary studies, strong weighting was given to members of the NfsA and NfsB families in the final library, these contributing 20 enzymes and 12 enzymes respectively (Figure 4-1). Unless otherwise 
stated enzymes in this library were expressed in the previously described E. coli tester strain SOS-R2 using the expression vector pUCX

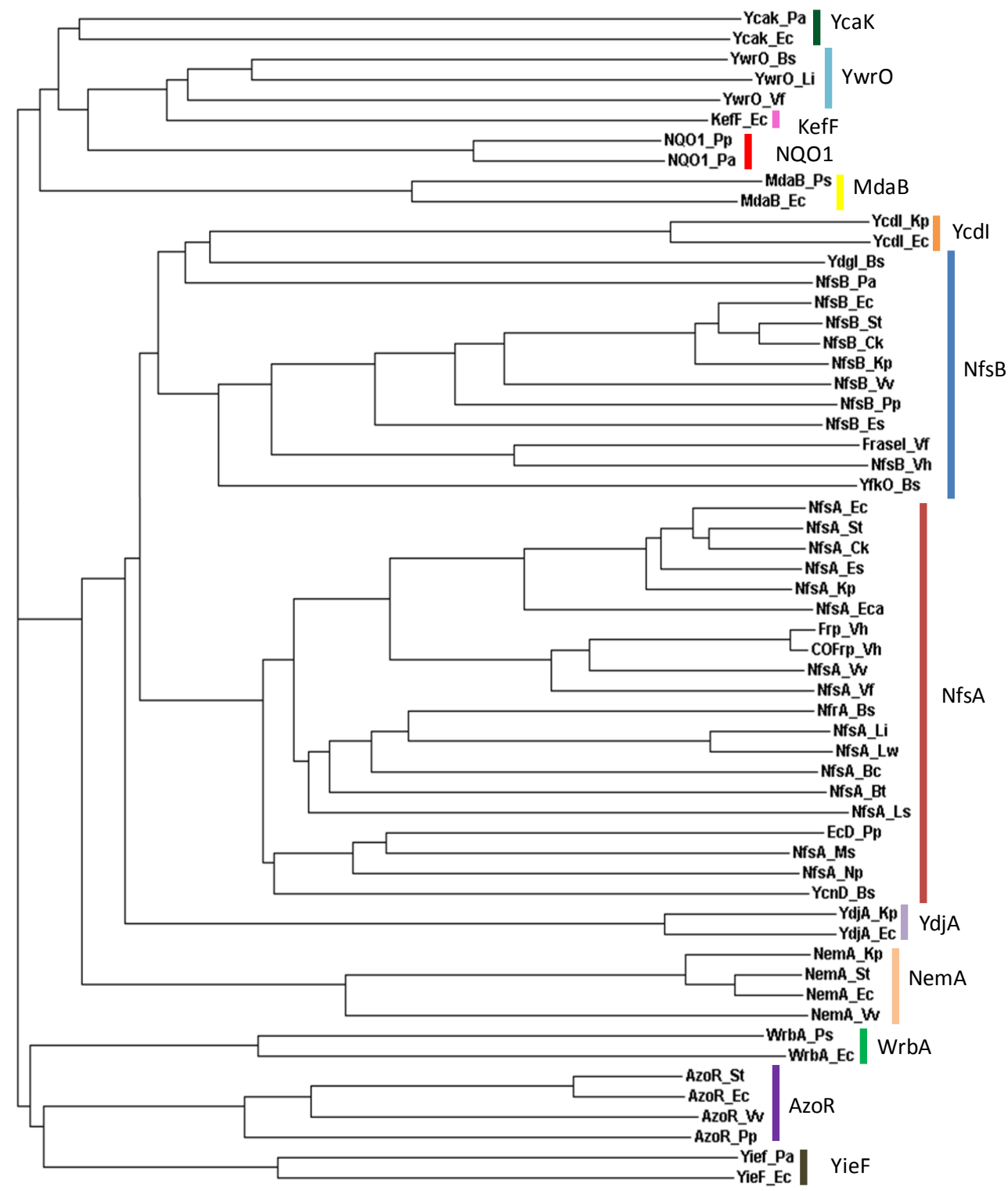

Figure 4-1 Phylogeny tree of the 58 oxidoreductases in the expanded NTR candidate library. Multiple sequence alignment was performed using Clustal Omega (http://www.ebi.ac.uk/Tools/msa/clustalo/) on default settings. Bootstrap values are not shown for clarity of presentation. Grouping of enzymes into families was based on the degree of shared sequence identity with the closest $E$. coli representative for all families ( $>24 \%$ protein sequence identity) except for YwrO and NQO1, which were assigned family names based on their shared identity with $B$. amyloliquefaciens YwrO and Rattus norvegicus NQO1, respectively. Individual NTR names indicate enzyme family, except in some instances where names previously reported in the literature have been used i.e. YcnD_Bs. This is followed by a two letter abbreviation for the genus and species of bacteria the enzyme originated from i.e. Bs- Bacillus subtilis, Vf- Vibrio fischeri (exception - Eca for Erwinia carotovora, to distinguish this from Ec for E. coli). For specific bacterial strain used see Table 2-3 and for levels of amino acid identity and similarity and accession numbers see Table 2-9. CO-Frp and Frp are slight variants of the same enzyme, derived from two different $V$. harveyi isolates. 
A handful of the NTR candidates included in this library have been described previously. In the NfsA family this includes the NADPH dependent FMN reductase Frp from $V$. harveyi, -the first "NfsA" to be crystallised (Tanner et al., 1996). In addition to a demonstrated ability to reduce nitro-compounds (Zenno et al., 1998) it is also a chromate reductase (Kwak et al., 2003). B. subtilis contains two different NfsA family members NfrA_Bs and YcnD_Bs, which share only 36\% amino acid identity. Both enzymes have X-ray crystal structures determined (Cortial et al., 2010; Morokutti et al., 2005) . It has been briefly noted that the 2-NI azomycin can be reduced by NfrA_Bs (Cortial et al., 2010); to my knowledge the only 2-NI activity ever previously described for a bacterial NTR. YcnD_Bs, which shares 35\% amino acid identity with NfsA_Ec, also shares a very similar protein structure - reported as having a root-mean-square deviation of $1.2 \AA$ over 220 aligned protein backbone carbon atoms when compared to the NfsA_Ec crystal structure 1IDT (Morokutti et al., 2005). EcD from Pseudomonas putida has been characterised as chromate reductase (Gonzalez et al., 2005a). Both NfsA and NfsB homologues from $S$. typhimurium have received attention due to their ability to activate the toxicity of nitro compounds in the S. typhimurium based Ames mutagenicity assay (Nokhbeh et al., 2002; Watanabe et al., 1998) and NfsB_St has been further characterised as an enoate reductase (Yanto et al., 2010). Additional NfsB family members that have been characterized include FRaseI_Vf, the major flavin reductase of the bioluminescent $V$. fischeri species (Zenno et al., 1994) - the structure of which (Koike et al., 1998) shows only a root-mean-square deviation of $1.4 \AA$ over backbone carbon atoms compared to NfsB_Ec (Parkinson et al., 2000).

\subsection{Aims}

- Interrogate the ability of enzymes from the expanded NTR candidate library to activate the 2-NI PET-capable imaging agents EF5 and F-miso, to identify any NTRs with greater activity than NfsA_Ec

- Compare different microplate based screening methodologies used for the evaluation of NTR activity with 2-NI compounds

- Determine the kinetic parameters of 2-NI active enzymes and compare these to the results of microplate based screens, to shed light on the key parameters measured in each screen 


\subsection{Results}

\subsubsection{Screening of the expanded NTR candidate library by monitoring 2-NI dependent growth inhibition}

\subsubsection{EF5 mediated growth inhibition assay}

The ability of enzymes within the expanded NTR candidate library to metabolise EF5 was first assessed by monitoring EF5 dependent growth inhibition of NTR over expression strains. Rather than performing detailed $\mathrm{IC}_{50}$ measurements for each of the 58 NTR candidate strains across a range of EF5 concentrations, the EF5 metabolism of the entire NTR library was first measured by monitoring growth inhibition over four hours in the presence of either $130 \mu \mathrm{M}$ or $400 \mu \mathrm{M}$ EF5 (130 $\mu \mathrm{M}$ representing a value close to the previously determined NfsA_Ec EF5 IC 50 ) (Figure 4-2). Reduction of EF5 was observed to be a general property of all NfsA family members, with the exception of the most distal NfsA_Ec relative, NfsA_Ms. At the higher EF5 concentration it was apparent that one NfsB family member, YfkO_Bs (29\% shared amino acid identity to NfsB_Ec), could metabolise the compound with substantially higher efficiency than the remaining NfsB enzymes. 
A)

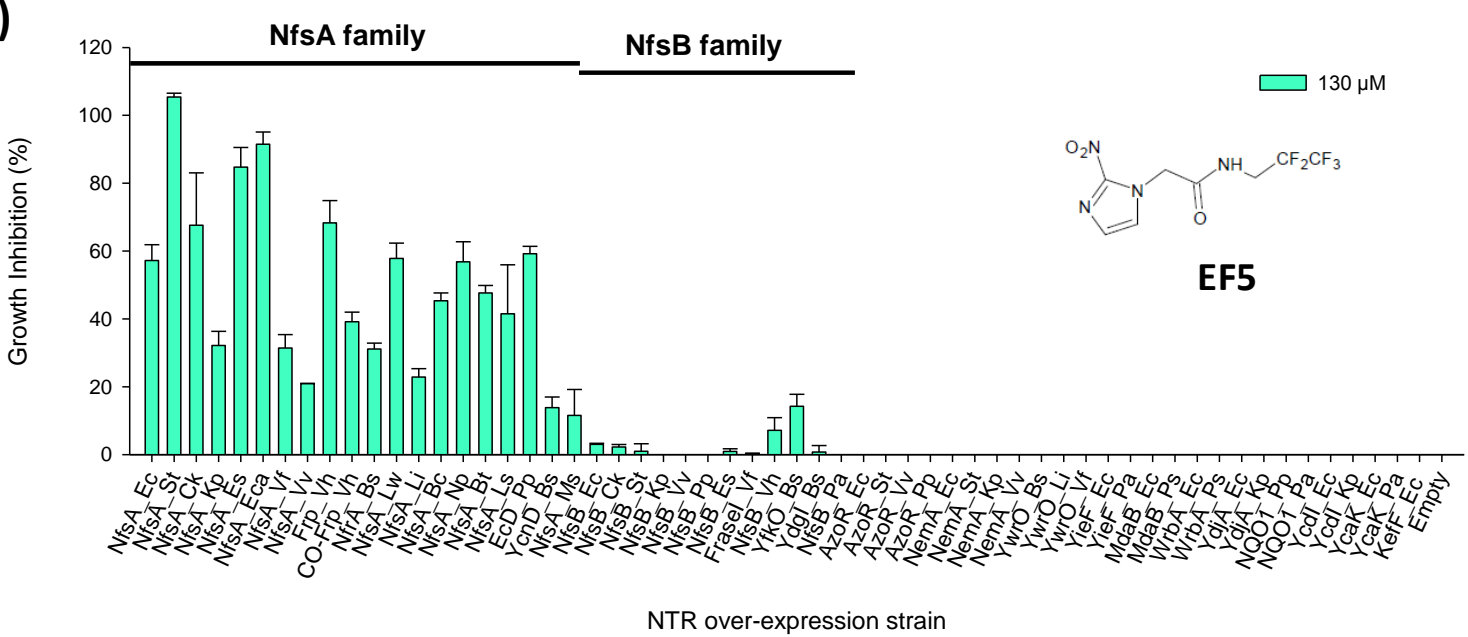

B)

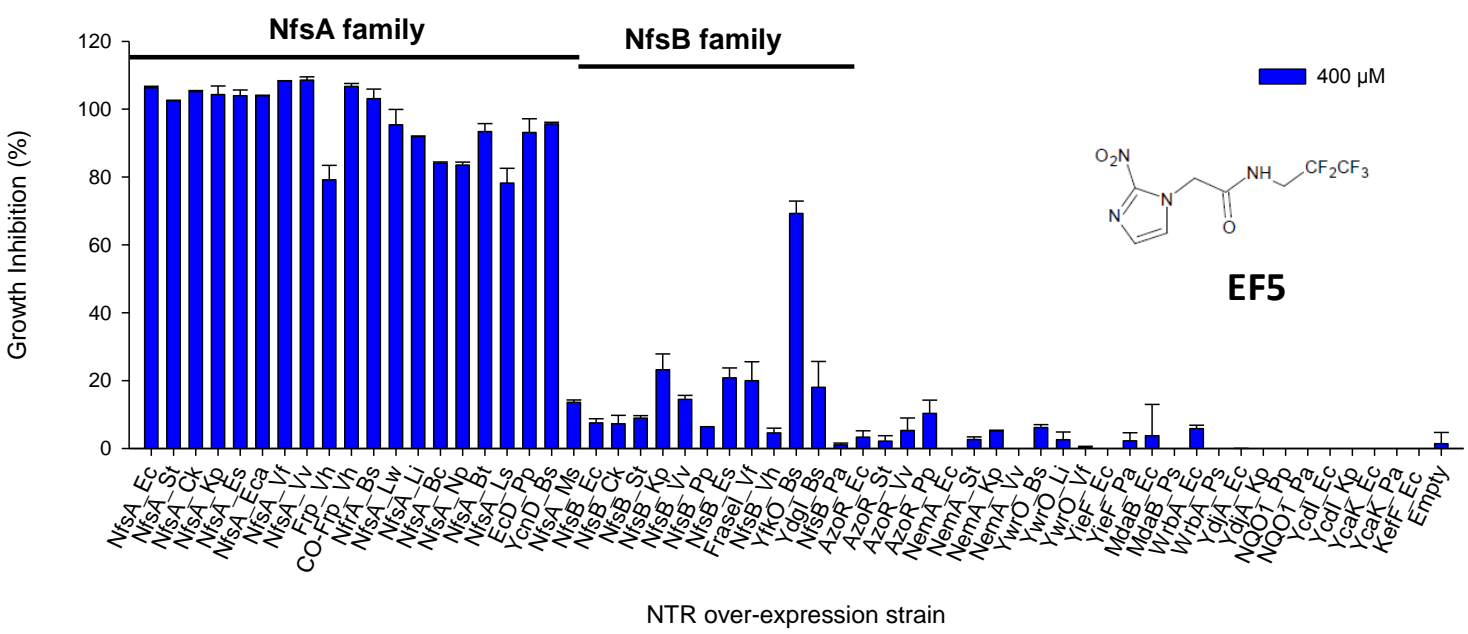

Figure 4-2 EF5 mediated growth inhibition of SOS-R2 E.coli NTR over-expression strains. Mid-exponential phase over-expression strains were challenged in 384 well plate format A) 130 $\mu \mathrm{M}$ or B) $400 \mu \mathrm{M}$ EF5for 4 hours. Growth was measured as culture turbidity $\left(\mathrm{OD}_{600}\right)$ pre and post drug challenge. Growth inhibition is presented as the percentage decrease in the growth of challenged cultures relative to the unchallenged controls for each strain. Data are the average of three independent experiments \pm S.E.M.

\subsubsection{IC $_{50}$ values for selected $E$. coli NTR over-expression strains challenged with EF5}

Next the EF5 IC $_{50}$ values were determined for the 20 NTR over-expression strains which had shown pronounced growth inhibition in the presence of $400 \mu \mathrm{M}$ EF5 (Figure 4-3). It appears that, as determined by this measure, NfsA_Ec is actually one of the most effective NTRs for metabolism of EF5, although the NfsA_St over-expression strain did exhibit the lowest EF5 IC 50 (NfsA_Ec $127 \mu \mathrm{M}$ vs NfsA_St $70 \mu \mathrm{M}$ ). 


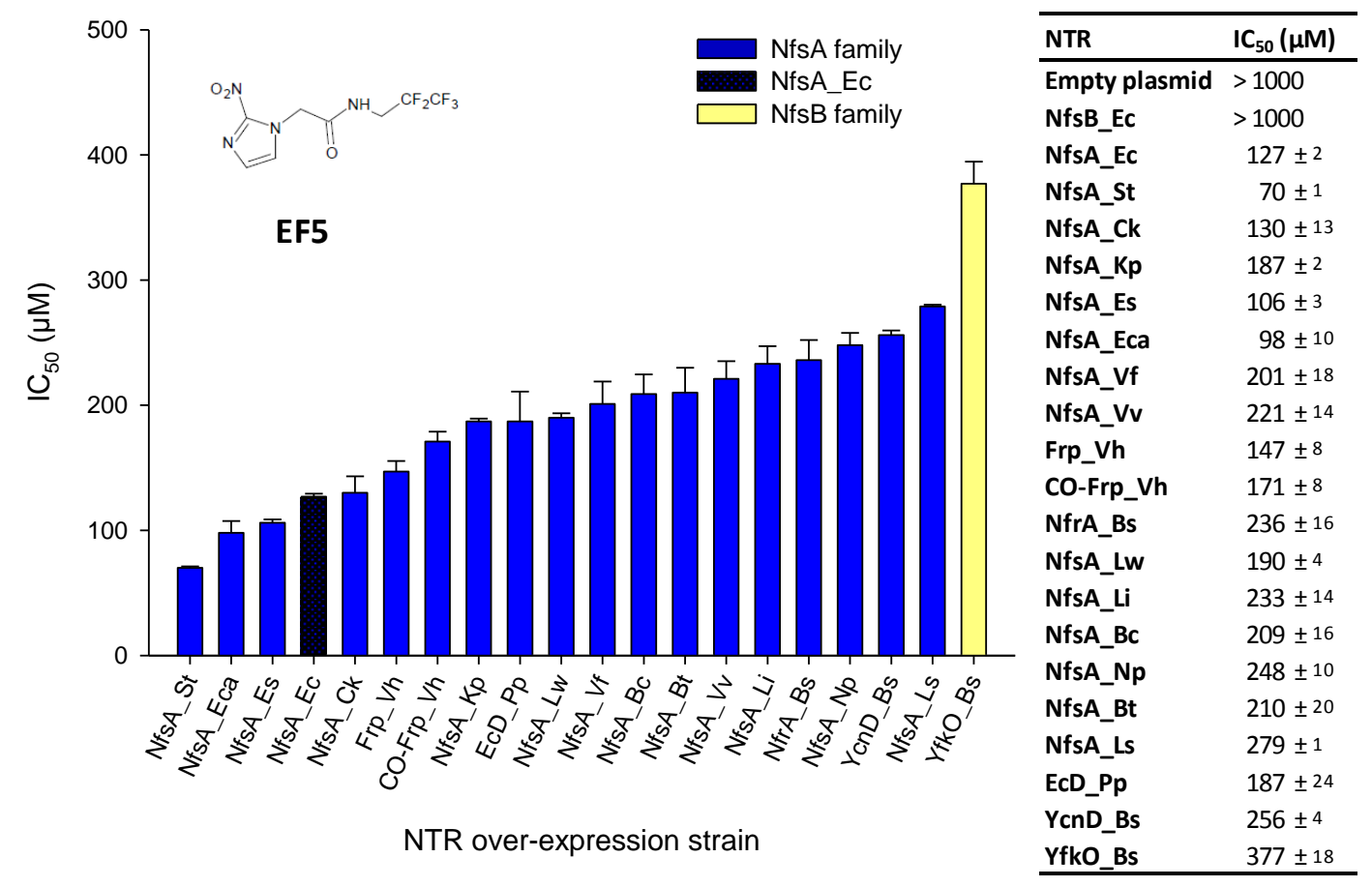

Figure 4-3 EF5 IC I0 $_{0}$ values for SOS-R2 E. coli NTR over-expression strains. Drug dependent growth inhibition was monitored by measuring strain turbidity $\left(\mathrm{OD}_{600}\right)$ pre and post four hour incubation with a two-fold dilution series of EF5. Percentage growth relative to unchallenged controls was determined and SigmaPlot ${ }^{\mathrm{TM}} 10.0$ used to calculate the $\mathrm{IC}_{50}$. Data are the average of three independent experiments \pm SEM. Graphed strains are ordered by increasing $\mathrm{IC}_{50}$.

\subsubsection{F-miso mediated growth inhibition and $\mathbf{I C}_{\mathbf{5 0}}$ determination}

Although screening efforts were primarily focused on EF5 for the reasons discussed in section 1.1, the metabolism of the prototypical 2-NI imaging agent F-miso was also assessed. This was again done by monitoring growth inhibition at $130 \mu \mathrm{M}$ and $400 \mu \mathrm{M}$ (Figure 4-4) and F-miso $\mathrm{IC}_{50}$ values were determined for all NfsA and NfsB family members, with the exception of the non-sensitising NfsB_Pa (Figure 4-5). 


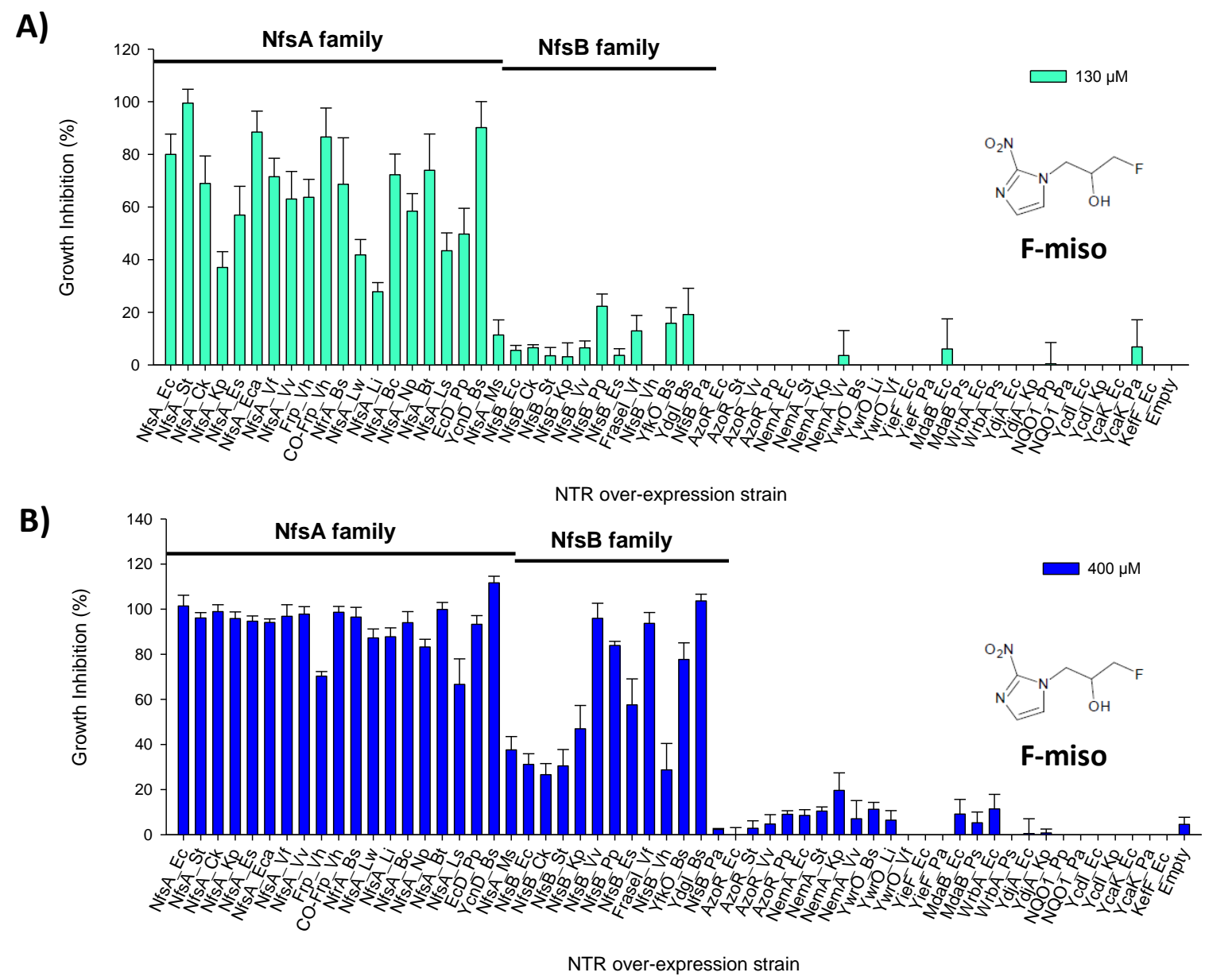

Figure 4-4 F-miso mediated growth inhibition of SOS-R2 E.coli NTR over-expression strains. Mid-exponential phase over-expression strains were challenged in 384 well plate format A) $130 \mu \mathrm{M}$ or B) $400 \mu \mathrm{M}$ F-miso for 4 hours. Growth was measured as culture turbidity $\left(\mathrm{OD}_{600}\right)$ pre and post drug challenge. Growth inhibition is presented as the percentage decrease in the growth of challenged cultures relative to the unchallenged controls for each strain. Data are the average of three independent experiments \pm S.E.M.

As was the case with EF5, all NfsA family members except NfsA_Ms could readily metabolise F-miso. Only three NTRs (YcnD_Bs, NfsA_Eca and NfsA_St) conferred Fmiso $\mathrm{IC}_{50}$ values lower than NfsA_Ec.

It is possible that the smaller side chain of F-miso (compared to EF5) may facilitate its reduction by some NfsB family members, with five NfsB like enzymes (YdgI_Bs, FraseI_Vf, NfsB_Vv, YfkO_Bs and NfsB_Pp) conferring F-miso $\mathrm{IC}_{50}$ values that are only 2-3 fold higher than that of NfsA_Ec (though still 4-6 fold higher than that of the "best” NfsA- NfsA_St). 


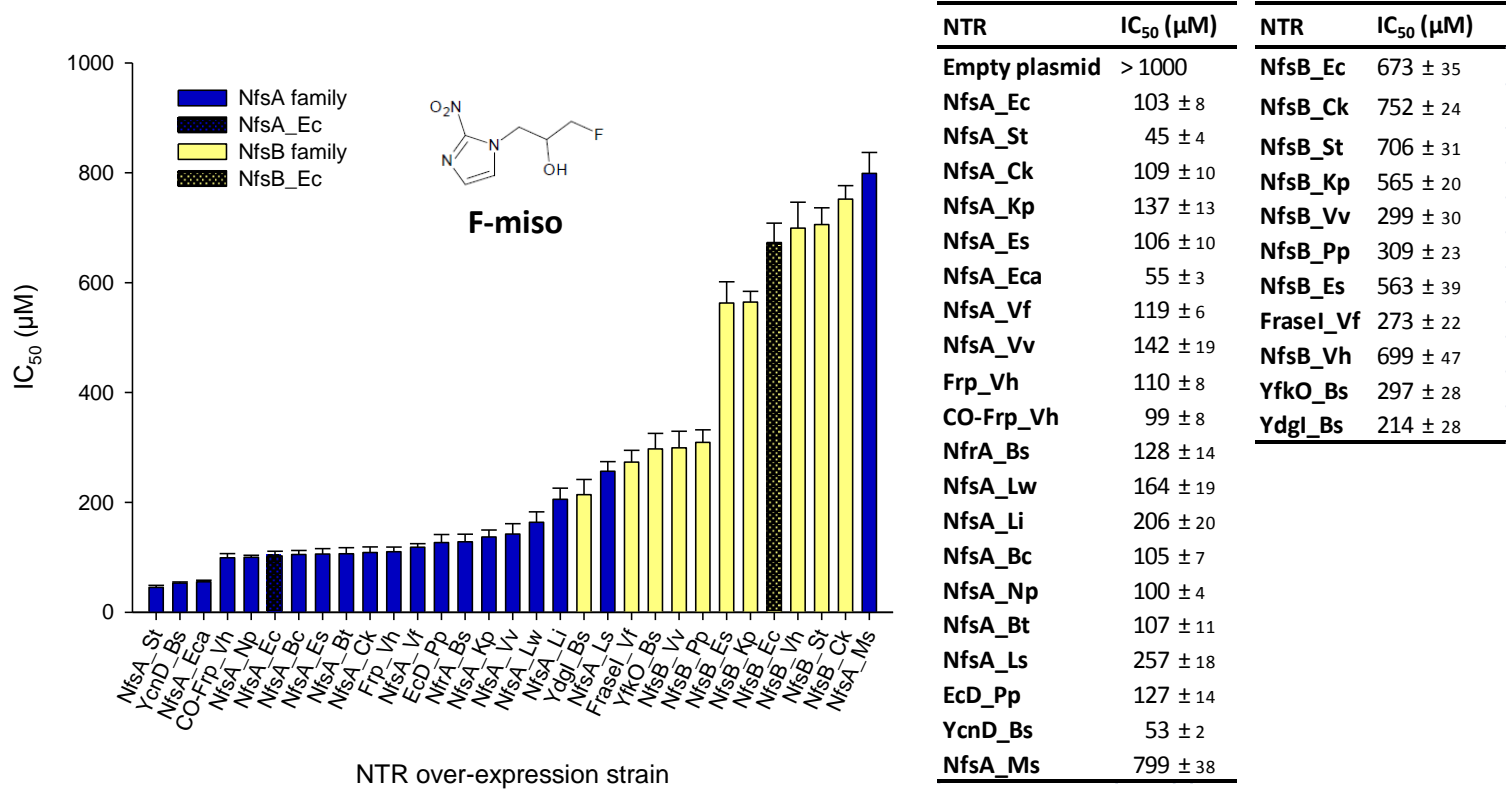

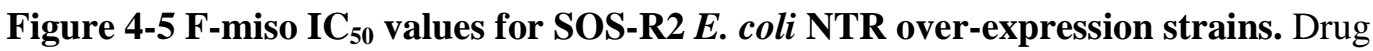
dependent growth inhibition was monitored by measuring strain turbidity $\left(\mathrm{OD}_{600}\right)$ pre and post four hour incubation with a two-fold dilution series of F-miso. Percentage growth relative to unchallenged controls was determined and SigmaPlot ${ }^{\mathrm{TM}} 10.0$ used to calculate the $\mathrm{IC}_{50}$. Data are the average of three independent experiments \pm SEM. Graphed strains are ordered by increasing $\mathrm{IC}_{50}$.

4.3.2 Screening of the expanded NTR candidate library using a RB6145 SOS assay

Our laboratory generally screens for NTR activity with DNA damaging prodrugs using the medium throughput microplate based SOS assay (Prosser et al., 2010; Swe et al., 2012). As demonstrated in Chapter 3, RB6145 is the only 2-NI compound in our collection that elicits a measurable SOS response following NTR-catalysed activation. While this is not itself a PET-capable molecule, for enzyme engineering studies it could potentially be useful to increase screening throughput with this assay. To determine whether RB6145 might reasonably predict metabolism of the other 2-NI molecules in our collection, the expanded NTR candidate library was assessed by RB6145 SOS assay (Figure 4-6). The activities of the entire expanded NTR candidate library with RB6145 were then correlated with EF5 and F-miso in turn (Figure 4-7). It was shown that activity with RB6145 correlated strongly with metabolism of both EF5 $\left(\mathrm{R}^{2}=0.79\right)$ and F-miso $\left(\mathrm{R}^{2}=0.76\right)$ - and in particular, that every NTR with avid imaging agent metabolism also generated an SOS reponse post challenge with RB6145 


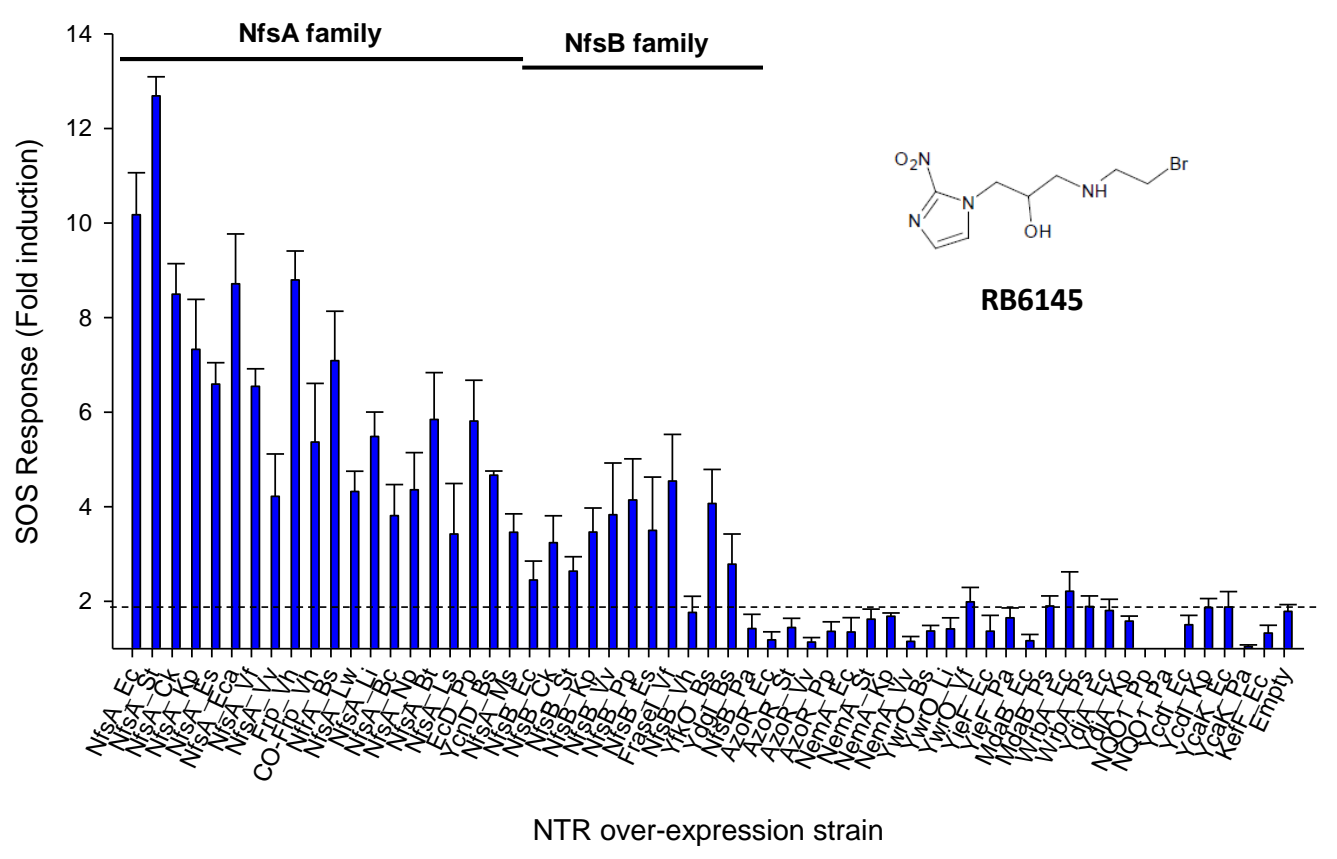

Figure 4-6 RB6145 mediated induction of the SOS response $E$. coli SOS-R2 NTR overexpression strains. Over-expression strains were grown in 96 well microplates and challenged with indicated concentration of RB6145 for 4 hours prior to quantification of SOS response by $\beta$ galactosidase assay. Fold induction is derived from the Miller units recorded on drug exposure divided by those of unchallenged control wells. Data are the average of two independent experiments $\pm \mathrm{SEM}$.

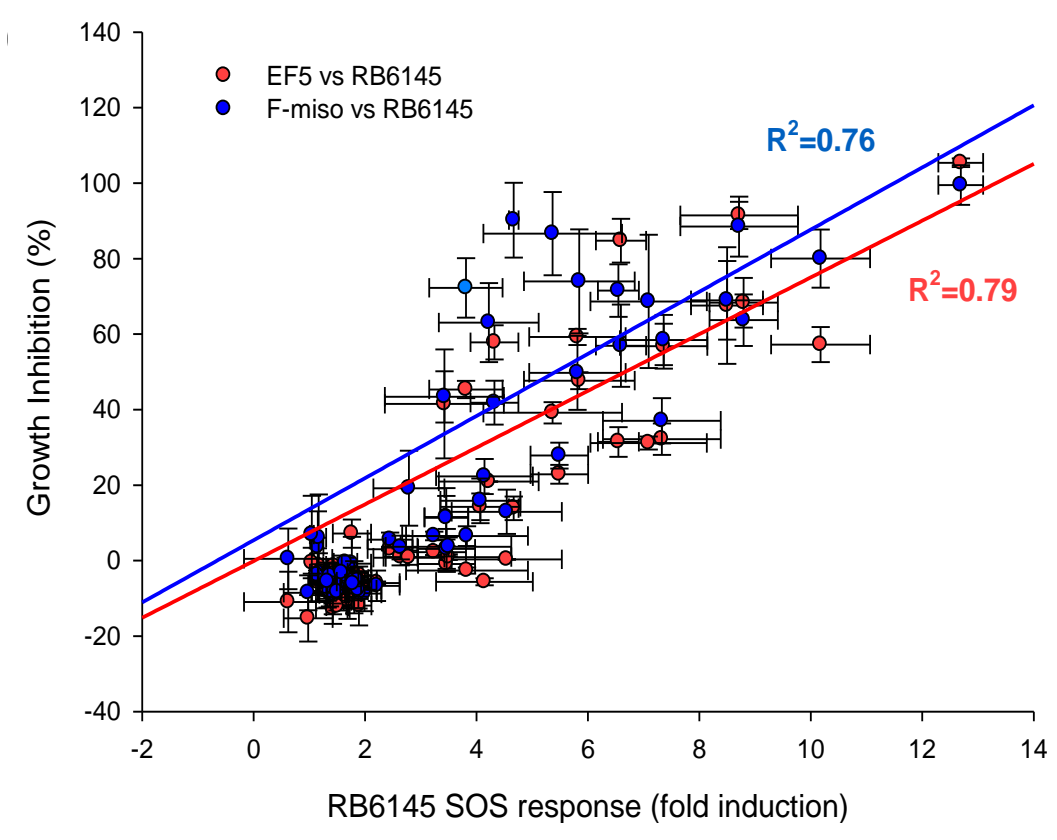

Figure 4-7 Linear correlation between the SOS response induced by RB6145 and the growth inhibitory effects of EF5 and F-miso in E. coli SOS-R2 NTR over-expression cultures. Response of the full library to $4 \mu \mathrm{M}$ RB6145 SOS assay (Data from Figure 4-6) and $130 \mu \mathrm{M}$ EF5 and F-miso growth inhibition assay (Figure 4-2 and Figure 4-4 respectively). 


\subsubsection{Evaluation of EF5 metabolism using the NADPH depletion assay}

As an additional screen the EF5 dependent NADPH oxidation by cell lysates of NTR over-expression cultures was measured using the NADPH depletion assay. First the activity of the entire expanded NTR library was assessed as seen in Figure 4-8 A. It was apparent that, over the time that the assay was allowed to progress, eight of the more active NTRs (marked by * in Figure 4-8 A) had when incubated with EF5entirely depleted all NADPH supplied. To further distinguish the relative activities of these NTRs they were re-assayed using less cell lysate and a shorter reaction time (Figure 4-8 B).
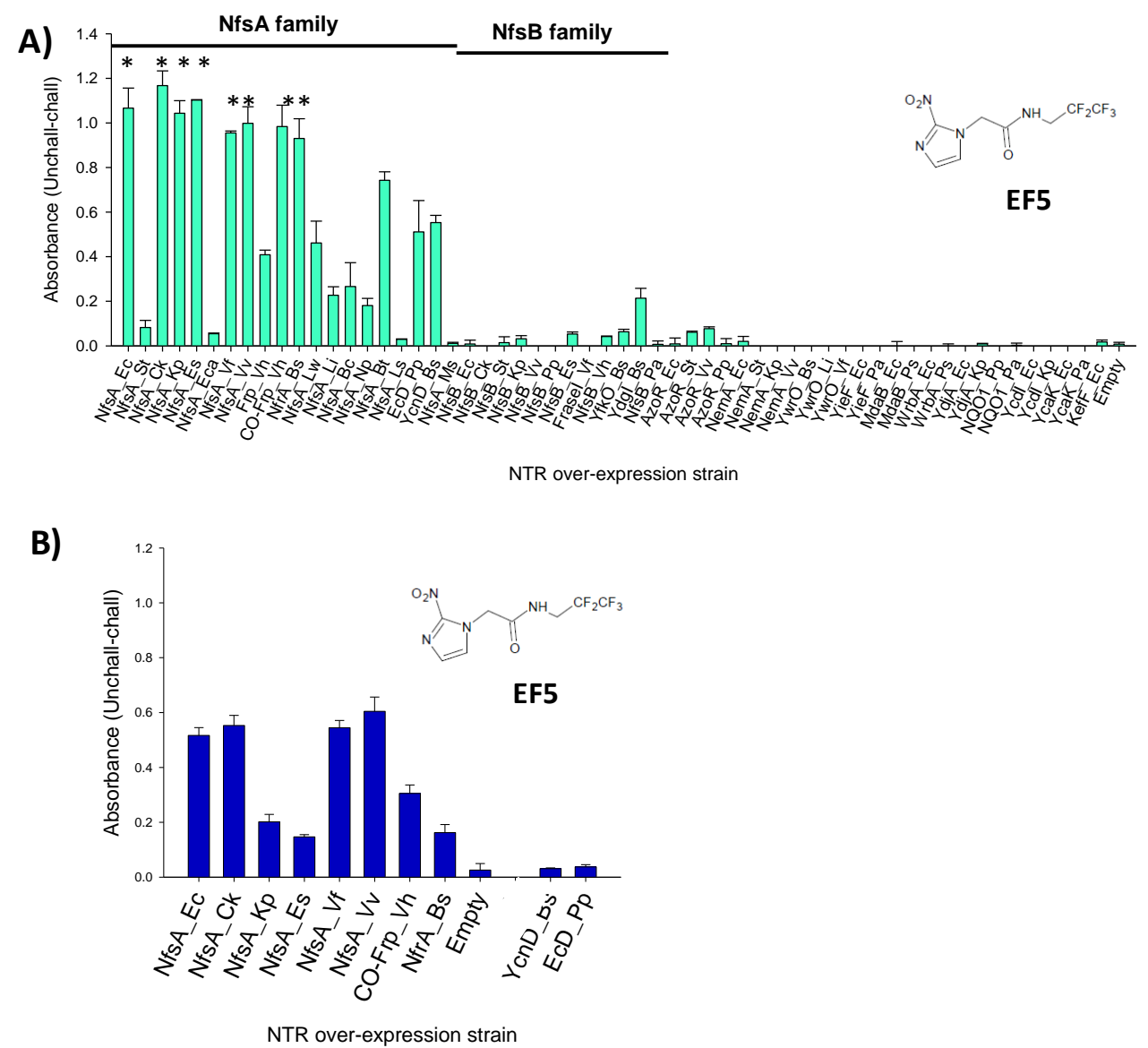

Figure 4-8 NADPH consumption by SOS-R2:ntr cell lysates in the presence of EF5. Crude cell lysates were incubated with $200 \mu \mathrm{M}$ NADPH and $150 \mu \mathrm{M}$ EF5. Addition of NBT post-incubation yielded formazan dye in proportion to the remaining $N A D(P) H$, quantified by measuring absorbance at OD590. Plotted values indicate the extent of EF5 metabolism by each NTR over-expression strain and were derived by subtracting the OD590 of EF5 challenged lysates (chall) from the OD590 of unchallenged duplicate controls (unchall). A) Assay run for 30 mins using $12.5 \mu \mathrm{L}$ cell lysate, the eight NTR over-expression strains which oxidised all supplied NADPH in the presence of EF5 are marked *. Data are the average of three independent assays \pm SEM B) Assay run for 10 mins using $5 \mu \mathrm{L}$ cell lysate - showing the * NTR over-expression strains plus empty plasmid, YcnD_Bs and Ecd_Pp controls. Data are the average of four independent assays \pm SEM. 


\subsubsection{Inconsistencies between the growth inhibition and NADPH depletion} assays

As determined by both the $\mathrm{IC}_{50}$ assay and NADPH depletion assay, only NfsA family members show avid metabolism of EF5. However when examining the NTRs within this family it became apparent that there is little correlation between the two assays $\left(\mathrm{R}^{2}=0.014\right.$ for a linear correlation between the activities of NfsA family members as determined by $\mathrm{IC}_{50}$ and NADPH depletion assays -result not shown). The greatest outliers in this correlation were the enzymes NfsA_St and NfsA_Eca (although even when these NTRs were removed from the correlation $\mathrm{R}^{2}$ rose to only 0.19 - result not shown). As measured by $\mathrm{IC}_{50}$ assay, both NTRs show EF5 metabolism comparable or better than that of NfsA_Ec; yet when using the NADPH depletion assay negligible EF5 metabolism is detected. This was not an issue of cofactor preference - when the expanded NTR library was re-assayed using NADH, again little cofactor depletion was observed for these NTRs (results not shown). A low level of EF5 dependent NADPH depletion by NfsA_St and NfsA_Eca over-expression cultures can eventually be detected, but this requires leaving the cell lysate to incubate with EF5 for > 60 mins (results not shown).

\subsubsection{Evaluation of EF5 reduction by purified His6-tagged NTRs}

In an effort to shed some light on this phenomenon, the apparent kinetic parameters of EF5 reduction by purified His6-tagged NTRs were determined as previously described. The enzymes successfully purified and tested were $12 \mathrm{NfsA}$ homologues spanning a range of EF5 activities, including NfsA_St; and the NfsB family members NfsB_Ec and YfkO_Bs (Table 4-1) (purification of NfsA_Eca was attempted also, using either autoinduction media or low temperature LBK supplemented with betaine and sorbitol, but in either case the soluble expression of protein was not sufficient to allow purification).

When examining the kinetic parameters of EF5 reduction by the NfsA family members it can be seen that the apparent $K_{m}$ values for all of the NTRs - with the exception of NfsA_St - span only a $\sim$ three fold range $($ NfsA_Bt $=150 \mu \mathrm{M}$, NfsA_Vf $=420 \mu \mathrm{M})$ and the apparent $k_{\text {cat }}$ only a $\sim$ fivefold range $\left(\mathrm{NfsA} \_\mathrm{Bt}=3.4 \mathrm{~s}^{-1}\right.$, NfsA_Vv $\left.=16.9 \mathrm{~s}^{-1}\right)$. In comparison NfsA_St shows a much lower apparent $k_{c a t}$ and $K_{m}$ (respectively $\sim 130$ and $\sim 17$ fold lower than those of NfsA_Ec). It was determined that the use of the detergent Bugbuster® for cell lysis was not adversely affecting the activity of the purified His6tagged NfsA_St as NfsA_St purified after performing cell lysis using a French pressure 
cell press did not show faster catalytic turnover of either EF5 or the prodrug substrate PR104A (results not shown).

The substantially lower apparent $k_{\text {cat }}$ of NfsA_St results in it having the lowest specificity constant of all the NfsA homologues tested, even though it was the most active enzyme in vivo as determined by EF5 dependent E. coli growth inhibition (Figure 4-3). This suggested that enzyme $k_{\mathrm{cat}}$ might be the dominant factor influencing results of the NADPH depletion assay; but that it might have less influence in the 2-NI dependent growth inhibition assays (a hypothesis that makes some intuitive sense given the large concentrations of substrate added in the NADPH depletion assay).

\begin{tabular}{lcrc}
\hline NTR & $\boldsymbol{K}_{\boldsymbol{m}}\left(\boldsymbol{\mu \mathrm { M } ) ^ { \mathrm { a } }}\right.$ & $\boldsymbol{k}_{\text {cat }}\left(\mathbf{s}^{-1}\right)^{\mathrm{a}}$ & $\begin{array}{c}\boldsymbol{k}_{\text {cat }} / \boldsymbol{K}_{\boldsymbol{m}} \\
\left(\mathrm{mM}^{-1} \mathbf{s}^{-1}\right)\end{array}$ \\
\hline NfsA_Ec & $160 \pm 10$ & $12.1 \pm 0.2$ & 76 \\
NfsA_St & $9 \pm 1$ & $0.1 \pm 0.0$ & 11 \\
NfsA_Ck & $210 \pm 26$ & $14.9 \pm 0.6$ & 71 \\
NfsA_Kp & $290 \pm 23$ & $9.4 \pm 0.3$ & 32 \\
NfsA_Es & $150 \pm 21$ & $9.0 \pm 0.4$ & 60 \\
NfsA_Vf & $420 \pm 45$ & $11.0 \pm 0.5$ & 26 \\
NfsA_Vv & $290 \pm 45$ & $16.9 \pm 1.4$ & 58 \\
Frp_Vh & $140 \pm 6$ & $4.9 \pm 0.6$ & 35 \\
CO-Frp_Vh & $200 \pm 30$ & $11.5 \pm 0.5$ & 58 \\
NfrA_Bs & $290 \pm 15$ & $6.8 \pm 0.1$ & 23 \\
NfsA_Bt & $150 \pm 17$ & $3.4 \pm 0.1$ & 22 \\
YcnD_Bs & $380 \pm 35$ & $5.6 \pm 0.4$ & 15 \\
& & & \\
NfsB_Ec & $1800 \pm 840$ & $0.4 \pm 0.1$ & 0.20 \\
YfkO_Bs & $1400 \pm 320$ & $3.5 \pm 0.5$ & 3 \\
\hline
\end{tabular}

a apparent $K_{m}$ and $k_{c a t}$ as determined at $0.20 \mathrm{mMNADPH}$

Table 4-1 Kinetic parameters for the reduction of EF5 by purified His6-tagged NTRs. Rates of reduction at varying concentrations of EF5 and a fixed concentration of excess NADPH $(0.20$ $\mathrm{mM}$ ) were monitored at $340 \mathrm{~nm}$, rates measured in quadruplicate. Apparent $K_{m}$ and $k_{\text {cat }}$ derived using Sigmaplot 10.0 (Systat Software Inc., Richmond, CA). Extinction coefficient used was $19,000 \mathrm{M}^{-1} \mathrm{~cm}^{-1}$ for explanation of determination see section 2.11.1. 


\subsubsection{The ability of the kinetic parameters of purified protein reduction of EF5 to predict the results of the NADPH depletion assay and EF5 dependent growth inhibition.}

To investigate the hypothesis that the NADPH depletion assay and EF5 IC 50 measurements were identifying different NTRs as the most active due to divergent enzyme kinetic parameters influencing each assay to a different degree, the results of each assay were compared with the apparent $k_{\text {cat,, }}$ apparent $K_{m}$ and specificity constant $\left(k_{\text {cat }} / K_{m}\right)$ for each purified enzyme with EF5 (Figure 4-9).

It can be seen that the activities determined by the NADPH depletion assay do not correlate well with either the apparent $K_{m}$ values or specificity constant for each NTR $\left(\mathrm{R}^{2}=0.16\right.$ and 0.46 respectively), but did correlate strongly with the apparent $k_{c a t}$ $\left(\mathrm{R}^{2}=0.82\right.$ ) (Figure 4-9A). An opposite scenario was observed for the $\mathrm{IC}_{50}$ assay, where $\mathrm{IC}_{50}$ values showed no correlation whatsoever with either the specificity constant or apparent $k_{\text {cat }}$ values $\left(\mathrm{R}^{2}=0.09\right.$ and 0.02 , respectively), but did show a moderate correlation with the apparent $K_{m}\left(\mathrm{R}^{2}=0.63\right.$; Figure 1-10B). 


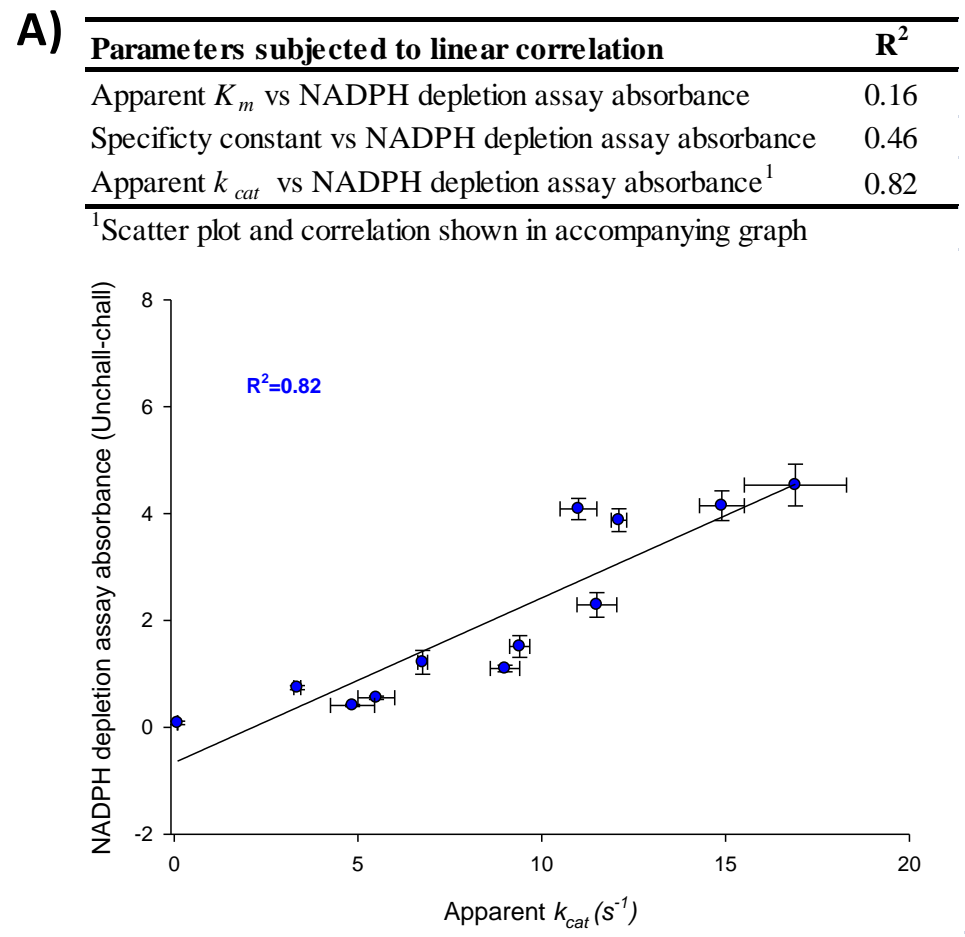

B) \begin{tabular}{lc}
\hline Parameters subjected to linear correlation & $\mathbf{R}^{\mathbf{2}}$ \\
\hline Apparent $K_{m}$ vs $\mathrm{IC}_{50}{ }^{1}$ & 0.63 \\
Specificty constant vs $\mathrm{IC}_{50}$ & 0.09 \\
Apparent $k_{\text {cat }}$ vs $\mathrm{IC}_{50}$ & 0.02 \\
\hline
\end{tabular}

${ }^{1}$ Scatter plot and correlation shown in accompanying graph

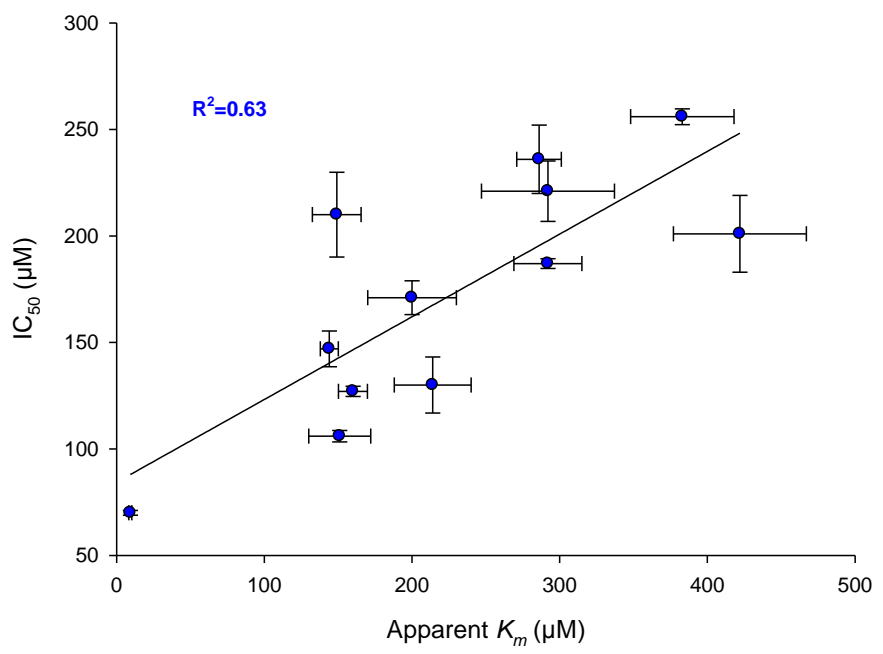

Figure 4-9 Linear correlations between kinetic parameters of NTR catalysed EF5 reduction and $\mathbf{E F 5}$ reduction as determined by $\mathrm{A}$ ) the $\mathrm{NADPH}$ depletion assay $\mathrm{B}$ ) $\mathrm{IC}_{50}$ values. Graphs shown represent the best correlation for each data set. The $\mathrm{R}^{2}$ values for the other relevant correlations are presented in inset tables. Kinetic parameters used are from Table 4-1, $\mathrm{IC}_{50}$ as presented in Figure 4-3 and NADPH depletion assay values as shown in Figure 4-8. To derive values for the eight NTRs with saturated activity in Figure 4-8A, NADPH depletion as determined in Figure 4-8B was taken and adjusted ( increased by a factor of 7.5) to take into account the shorter assay time and lower cell lysate volume. 


\subsection{Discussion}

\subsubsection{Screening of the expanded NTR library for 2-NI metabolism}

Work presented in this chapter assessed a range of oxidoreductase enzymes from an expanded NTR candidate library for the ability to metabolism 2-NI compounds. The primary driver behind this screening effort was to try and improve on NfsA_Ec as a preclinical candidate for a noninvasive PET imaging reporter gene.

To achieve this, the enzymes of the expanded NTR candidate library were first assessed for 2-NI metabolism by monitoring EF5 and F-miso dependent growth inhibition of $E$. coli NTR over-expression strains. Whereas efficient metabolism of EF5 was restricted to enzymes within the NfsA family, F-miso was found to have a wider activation profile, with a number of NfsB homologues (those within a range of $25-61 \%$ shared amino acid identity to NfsB_Ec) demonstrating levels of F-miso metabolism closer to that of the NfsA enzymes. While the lack of antibodies able to detect F-miso binding means we cannot further test NTR/F-miso imaging capabilities by immunohistochemistry, if in the future we are able to conduct a radiobiology based assessment of NTR dependent F-miso binding, then the activities of these NfsB family members could warrant further investigation.

When the NTR strains were ranked by decreasing 2-NI IC I0 $_{5}$, the NfsA_Ec strain was found to be one of the most sensitised, with only NfsA_St conferring a $\sim 2$ fold lower EF5 $\mathrm{IC}_{50}$, and only three NTRs showing a $\sim 2$ fold lower F-miso $\mathrm{IC}_{50}$. The library was further assessed by monitoring the RB6145 induced SOS response, yielding similar results. All NTRs that could metabolise an imaging agent could also metabolise RB6145, suggesting that RB6145 SOS assays could perhaps be used as a preliminary screening step for identifying NTRs that can metabolise 2-NIs. This observation was of particular significance for directed evolution work conducted later in this project, presented in Chapter Five.

Next the NADPH depletion assay was used to assess the activities of the expanded NTR candidate library with EF5. Surprisingly the results of this assay showed little correlation with the NTR activities determined by EF5 IC $_{50}$ measurement. The kinetic parameters for the reduction of EF5 by His6-tagged purified proteins were then determined for $12 \mathrm{NfsA}$ enzymes and compared to the results of the EF5 $\mathrm{IC}_{50}$ and NADPH depletion assays. The 
specificity constants of the purified protein reactions did not correlate particularly well with either assay (better for the NADPH depletion than the $\mathrm{IC}_{50}$ assay, $\mathrm{R}^{2}=0.46$ vs. $\mathrm{R}^{2}=0.09$ respectively). However when the individual kinetic parameters used to derive the specificity constant - the apparent $k_{\text {cat }}$ and $K_{m}$ - were compared to the results of the E. coli based screens, a possible explanation for the discrepancy between the results of the two screens began to emerge. The results of the NADPH depletion assay showed good correlation with the enzymes apparent $k_{c a t}$ i.e. the maximum theoretical rate of reduction $\left(\mathrm{R}^{2}=0.82\right)$. In contrast the $\mathrm{IC}_{50}$ values did not correlate at all with the apparent $k_{\text {cat }}$ but instead showed a moderate correlation with the apparent $K_{m}$ of the reaction; a parameter generally considered to indicate the affinity that an enzyme has for a particular substrate.

While somewhat speculative, these observations suggest that the outcome of each assay is primarily driven by a different aspect of NTR enzymatic activity. The $\mathrm{IC}_{50}$ values are a measure of the EF5 dependent toxicity generated as a result of sustained activity of the NTR over a period of four hours, at intracellular concentrations of EF5 that are presumably (due to the permeability barrier of the E. coli outer membrane and possibly the action of efflux pumps residing therein (Vaara, 1992)) far lower than those added to the extracellular growth medium. If the imaging agent substrate is substantially lower than the $K_{m}$, then $k_{c a t}$ would have less influence than it does at high substrate concentrations. In contrast, the NADPH depletion assay measures relatively short term metabolism by cell lysates at much higher EF5 concentrations, meaning the theoretical maximum rate of reaction should have a greater effect on results of the assay. While $k_{\text {cat }} / K_{m}$ is generally regarded as the most useful parameter to compare the activities of the same enzyme with different substrates, it has been shown that it can be misleading when used to compare the activities of different enzymes with the same substrate. An enzyme with a higher specificity constant but also a higher $K_{m}$ can, over certain (low) substrate concentrations actually catalyse a reaction more slowly than an enzyme that has a lower specificity constant but also a lower $K_{m}$ (Eisenthal et al., 2007). In short, the lower the available substrate concentration, the more important the $K_{m}$ parameter becomes.

A critical question here is which assay is most relevant to this project? Given the translational aspirations of this study, the most relevant assay is that which most reliably identifies NTRs effective in promoting 2-NI activation in transfected human cells. Our collaborators at the ACSRC attempted to address this issue for us. However, their efforts were complicated by a non-trivial technical concern. 


\subsubsection{Expression of selected members of the expanded NTR candidate library from HCT-116}

To assess the activities of the expanded NTR candidate library in human cells, 15 stably transfected NTR-expressing HCT-116 cell lines were generated by our collaborators at the ACSRC - encompassing nine NTRs from the NfsA family and six from the NfsB family. These were initially generated to assess metabolism of the prodrugs PR-104A and CB1954 (as presented in (Prosser et al., 2013)) but were also intended to evaluate metabolism of EF5.

Prior to testing HCT-116:ntr activity the level of NTR expression in each cell line was quantified. Monoclonal antibodies which can detect the expression of specific NTRs are only available for NfsA_Ec and NfsB_Ec. However the expression of the diverse NTRs tested here can be detected using Invitrogen Tag-On-Demand ${ }^{\mathrm{TM}}$ technology (Prosser et al., 2013; Syddall, 2009). NTRs genes cloned into the F279-V5 vector terminate in a TAG stop codon, immediately after which is C-terminus V5 epitope tag. NTR expression can thus be quantified by infecting a sample of each cell line with a TAG stop suppressor adenovirus (Tag-On-Demand ${ }^{\mathrm{TM}}$, Invitrogen, Carlsbad, CA), permitting read through of the TAG-terminated NTR gene to give an immunoreactive V5-epitope at the C-terminus of the expressed protein, which is then detected by western blot. One testing the expression levels of these NTRs a significant technical issue became apparent, in that the levels of stable and functional expression of the different NTRs from HCT-116 varied dramatically, with some NTRs showing no expression whatsoever Figure 4-10.

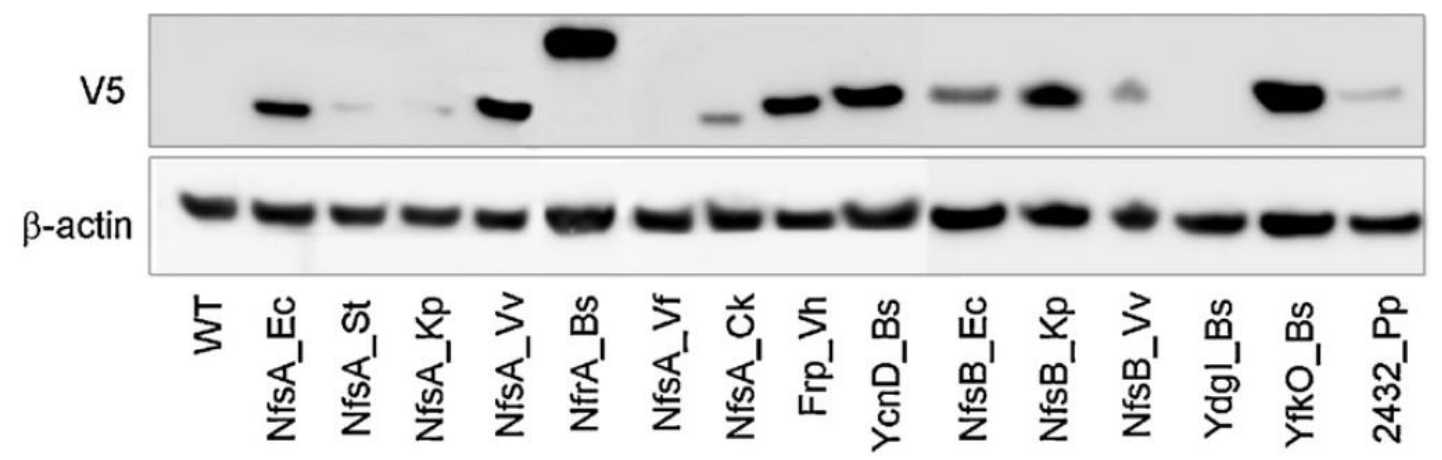

Figure 4-10 Expression and functional validation of top nitroreductase candidates in a human cancer cell line model. Expression of bacterial nitroreductase genes in stably transfected HCT-116 cells was determined by western blotting. Cells were infected with an adenovirus encoding a TAG suppressor tRNA that enables transient induction of C-terminal V5 tags (Tag-onDemand, Invitrogen, Carlsbad, CA; multiplicity of infection 50, 27 h). Reproduced with permission from (Prosser et al., 2013) 
Furthermore, initial levels of protein expression have been shown to sharply decrease for some NTRs over the time period that the cell lines are kept in culture (Christopher Guise and Alex Mowday, personal communication, (Mowday, 2010). This was unexpected as at the ACSRC long term stable expression of NfsA_Ec and NfsB_Ec had previously been observed over the course of multiple in vitro and in vivo experiments, via expression from different vector back bones (Syddall, 2009) and (for NfsB) on expression from different human tumour cell lines (Singleton et al., 2007). Moreover, to our knowledge no problems regarding expression of bacterial NTRs in human cells have previously been reported in the literature.

These issues greatly complicated assessment of NTR activity from HCT-116 cells. Alex Mowday did manage to test five NfsA family members (NfsA_Ec, NfsA_St, NfsA_Vv, NfrA_Bs and NfsA_Kp) for the ability to metabolise EF5. All cell lines except NfsA_Kp (which at time of testing showed only trace levels of expression as determined by western blot) showed some level of EF5 binding, as determined by flow cytometry of cells which had been incubated with EF5 and then exposed to a fluorescently labelled anti-EF5 antibody (Mowday, 2010). However, the variability in NTR expression levels from one cell line to the next and over time was such that it was not possible to draw sensible conclusions regarding relative activity levels in the human cellular environment.

At this point the exact reasons for these expression issues are unknown but possible theories will be advanced in later chapters of this thesis. It should be noted that while the variable and time dependent expression levels complicate assessment of NTR activity in our current pre-clinical models, which rely on stably transfected HCT-116 cell lines, it does not necessarily follow that NTRs unable to be stably expressed in HCT-116 are unsuitable for use in GDEPT. For example, viral GDEPT would be expected to be more akin to a transient expression scenario; whereas vectors such as Clostridium or Salmonella require stable NTR expression, but from a bacterial cell. Thus, these issues do point to the need to assess different NTRs in the context of the vector intended for patient therapeutic use, as these vectors will have different expression requirements.

\subsubsection{Conclusions regarding further $E$. coli based screening}

The next step in this research was a directed evolution study, in which mutant NTRs were generated and then screened for desired improvements in 2-NI metabolism. For this study it was important to determine that the screen used would accurately detect mutant NTRs 
with improved 2-NI metabolism in transfected human cells. The variable NTR expression from HCT-116 cells reported in this Discussion complicated our attempt to assess how accurately our E. coli based screens reported on this metabolism. However Alex Mowday did show that while the NADPH depletion assay and the purified protein $k_{\text {cat }}$ indicated negligible EF5 metabolism by NfsA_St, HCT-116 cell lines expressing NfsA_St could metabolise EF5 efficiently, at a level comparable to NfsA_Ec (of note NfsA_St was not expressed at a higher level than NfsA_Ec as determined by western blot) (Mowday, 2010). The EF5 metabolism of this NTR would not have been detected if we had solely screened the NTR expanded library using the NADPH depletion assay.

As such it was decided that future screening would focus on 2-NI dependent growth inhibition conferred by the mutant NTRs. This was based in part on our concerns regarding the inability of the NADPH depletion assay to detect NfsA_St metabolism; but also on indications from other parallel work in the Ackerley laboratory, that improvements in mutant NTRs ability to metabolise nitroaromatic prodrugs were often being driven by decreases in $K_{m}$ rather than increases in catalytic turnover rate (Swe et al., 2012). 


\section{Chapter 5: Directed evolution of NfsA_Ec for improved metabolism of 2- nitroimidazole imaging agents}

\subsection{Introduction}

\subsubsection{Enzyme engineering}

NTR catalysed reduction of xenobiotic 2-NI imaging agents is a serendipitous outcome of the enzymes' general substrate promiscuity, rather than a reaction which nature has specifically tailored them for through evolution. It is therefore highly likely that the reaction efficiency could be improved through lab based directed evolution of the NTRs. Essentially the idea is that a protein can be changed via random or targeted mutation of the DNA encoding it, followed by application of selection or screening strategies to identify variant proteins which display the desired property, i.e. increased metabolism of 2-NI imaging agents. Such enzyme engineering is often employed to improve proteins used as biocatalysts (Kumar and Singh, 2012) or therapeutics (Buchanan et al., 2012). An example of this with applications to PET imaging is the mutant HSV1-sr39tk, first identified as improved in metabolism of the prodrugs GCV and acyclovir (following screening of a mutant gene library generated via random mutagenesis of specific sections of HSV1-tk) (Black et al., 1996) and then fortuitously found to better activate a number of HSV1-tk reporter probes (Gambhir et al., 2000). In regard to NTR based GDEPT, a variant of YieF evolved for improved chromate reduction was found to also have serendipitously improved CB1954 metabolism (Barak et al., 2006) and two bacterial NTRs have been deliberately evolved for improved CB1954 reduction; FRase I by our research group (Swe et al., 2012) and an extensive evolution effort focused on NfsB_Ec carried out by Peter Searle's research group (Grove et al., 2003; Guise et al., 2007; Jaberipour et al., 2010). In ongoing work we have also evolved NfsA_Ec mutants for improved PR-104A activation, and this work will be overviewed in Chapter Seven.

This chapter will present efforts to evolve the NTR NfsA_Ec to improve its activation of 2-NI imaging agents. As shown previously, while the native activity of NfsA_Ec with EF5 does enable detection of in vivo NfsA_Ec expression from human cells using immunohistochemistry, there is still a level of hypoxia background present (Figure 3-11). At this point it is unknown if this background would substantially confound interpretation of PET images of NfsA_Ec-catalysed ${ }^{18} \mathrm{~F}$-2NI probe binding in a clinical context. The 
likelihood of this problem arising would be lessened by improvement of the ability of this NTR to metabolise EF5.

Three important considerations for any directed evolution study are how, and to what extent, to mutate the protein, and how to then identify any improved variants. The extent of protein mutation is often dictated by both the ease with which desirable variants can be identified and the extent of structural and functional knowledge available for that protein. This is because the majority of mutations will have deleterious or neutral effects on the relevant property and finding the rare improved clones becomes exponentially more challenging as additional sites are mutated and more variants are generated. This is not such a constraint if desired variants can be directly selected for or if an effective highthroughput screening strategy is available. However, as this is not the case for any screens of 2-NI activity developed thus far, it was decided to target mutagenesis to residues most likely to have maximal effect i.e. those around the active site. Such targeting is greatly facilitated by a solved X-ray crystal structure, as available for NfsA_Ec (Kobori et al., 2001), NfrA_Bs (Cortial et al., 2010), Frp_Vh (Tanner et al., 1996) and YcnD_Bs (Morokutti et al., 2005).

A thorough search for active site mutations that improve 2-NI metabolism was conducted by subjecting certain active site residues to site saturation mutagenesis (in which the effect of mutating a target residue to each of the other 19 standard amino acids is tested). Further improvements in 2-NI metabolism were then pursued by making multi-site mutants, as when individual beneficial residues are combined they can often have additive or even synergistic positive effects. One valid strategy for generating beneficial multi-site mutants is iterative saturation mutagenesis, a stepwise approach to accumulating beneficial mutations in which a gene containing a previously identified beneficial mutation(s) is iteratively used as the template for further site saturation mutagenesis at a different codon (Reetz and Carballeira, 2007). This stepwise approach does however require that all acquired mutations add benefit at every step of this process. An alternative approach that stands a greater chance of identifying synergistic mutations is to generate gene libraries which randomly combine multiple single mutations, followed by screening all resulting variants. The number of mutations to be combined can then be aligned with the efficiency of available screening strategies (Bornscheuer and Kazlauskas, 2011). 


\subsubsection{Docking of EF5 into the active site of NfsA_Ec}

NfsA_Ec was chosen as the NTR to be evolved due to its available crystal structure, relatively high native activity with both F-miso and EF5, and its demonstrated stability of expression in HCT-116 cells both in vitro and in vivo. As previously discussed, a lack of stable expression from HCT-116 does not necessarily preclude functional expression of an NTR from viral or bacterial clinical vectors, however to get to that stage it would be of immense value to first assess any improved mutants stably transfected HCT-116 preclinical models. EF5 was chosen for docking into the NfsA_Ec crystal structure as we are able to monitor NTR catalysed binding of EF5 in mammalian cells using immunohistochemistry.

Docking was performed by Dr Jack Flanagan at the ACSRC (Figure 5-1A and B). Two distinct orientations of EF5 binding in the active site were predicted. In one mode the pentafluorine tail of EF5 points back into the enzyme's binding pocket, whereas in the second the tail is closer to the FMN cofactor, forming a hydrogen bond between the EF5 amide hydrogen and the first hydroxyl group in the FMN phosphate side chain. From this docking Dr Flanagan generated a list of 12 NfsA_Ec residues likely to be involved in EF5 binding (Figure 5-1 C).

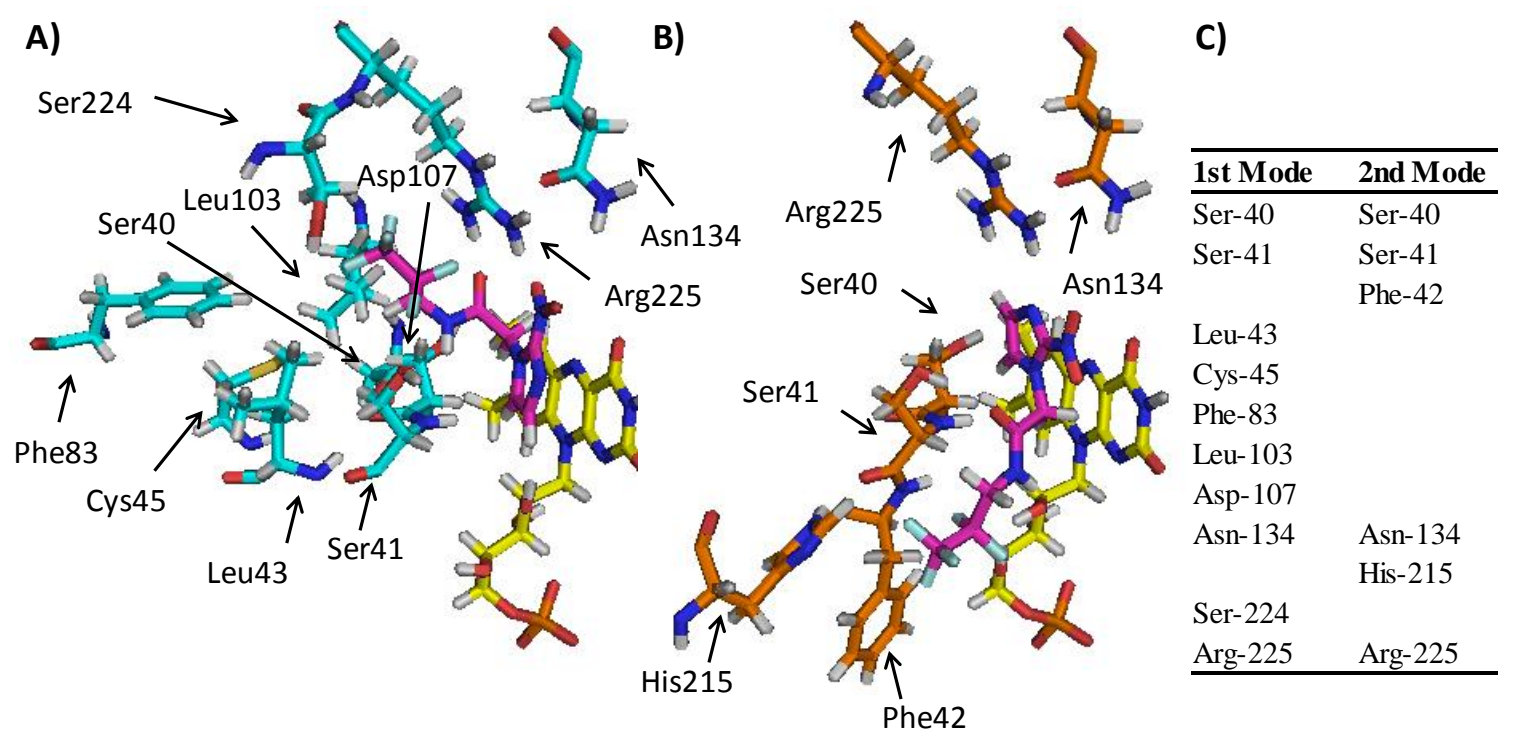

Figure 5-1 Docking of EF5 into the active site of solved crystal structure of NfsA_Ec Shown are A) the $1^{\text {st }}$ orientation and B) the $2^{\text {nd }}$ orientation of predicted EF5 binding. Docking was carried out by Dr Jack Flanagan at the ACSRC using Gold software (Hermes). Images generated in PyMOL (Schrödinger, LLC). and show both EF5 (pink carbons) the FMN co-factor (yellow carbons) and the protein residues (cyan or orange carbons) which were determined by Jack as involved in EF5 binding, either by direct proximity to EF5 or by packing directly behind those residues to hold them in place. C) The residues pictured in A) and B) summarised in table format. 


\subsubsection{Aims}

- Identify single residue changes in the NfsA_Ec active site that improve its reduction of F-miso and EF5

- Combine a selection of these mutations to identify additive or synergistic combinations of beneficial mutations

- Assess the ability of these mutants to reduce EF5 when stably expressed from the human cell line HCT116

- Investigate the suitability of a high-throughput FACS based screen for identifying clones with improved 2-NI metabolism from large multi-mutant libraries

\subsection{Results I: Directed evolution of NfsA_Ec using targeted site saturation mutagenesis}

\subsubsection{Construction of targeted single site mutagenesis libraries}

The 12 NfsA_Ec residues predicted to be involved in EF5 binding were individually subjected to site saturation mutagenesis. To do this, overlap PCR (as described in section 2.8) was used to generate an NfsA_Ec gene library which contained the degenerate bases NNK at the targeted mutation site. In the standard International Union of Biochemistry (IUB) degeneracy code $\mathrm{N}$ specifies that any DNA base can be incorporated at that position, while K indicates either thymine or guanine. Using NNK meant that the targeted mutation site contained any one of 32 different codons (instead of the normal 64) but could still code for all of the 20 common amino acids (and only one stop codon). This means only 94 individual colonies have to be screened to give $>95 \%$ certainty that every codon has been sampled at least once (Reetz and Carballeira, 2007).

These genes were cloned into the pUCX vector, and the resulting plasmids individually transformed into SOS-R2. For each library, PCR of 20 random colonies using NfsA_Ec gene specific primers was used to estimate the insert ratio (found to range from $50-90 \%$ per library- results not shown) and this was used to calculate the minimum number of colonies which had to be screened to give $>95 \%$ coverage for each library (105 - 188 per library). For each library a number of colonies not less than this minimum were picked into 96 well microplates containing NfsA_Ec wild type and empty plasmid controls, and these were subsequently screened for 2-NI metabolism. 


\subsubsection{Screening of 12 site saturation mutagenesis libraries for single residue mutations conferring increased 2-NI metabolism}

To minimise consumption of the supply-limited imaging agents it was decided to first target screening to clones that retained "generic" 2-NI metabolism. Activity with the more readily available 2-NI compound RB6145 can be assayed for by the medium throughput SOS assay using a very minimal drug quantity, and as previously shown (Figure 4-7) NTR activity with RB6145 was generally predictive of EF5 and F-miso metabolism.

All libraries were screened by RB6145 SOS assays in duplicate (results not shown) and clones which showed SOS induction equal or greater than the NfsA_Ec control were selected for further screening (ranging from 12\% - 32\% of clones for each library). These clones were tested by $150 \mu \mathrm{M}$ EF5 growth inhibition assays (results not shown). "Hits" from these assays were validated and quantified by repeated EF5 $\mathrm{IC}_{50}$ assays (Table 5-1) and the DNA sequences of the improved clones were determined. The activities of these mutants with F-miso were then assayed (results also shown in Table 5-1 ). Almost all the mutants selected for their improvement in EF5 reduction also showed an increased ability to metabolise F-miso, with only one mutation (N134W) detrimental and a handful of others (L43E, C45H, C45D and L103Q) giving neglible improvment.

Overall 47 clones with improved EF5 metabolism were identified and when sequenced found to contain 31 different mutations. These were spread over nine of the 12 sites targeted; no increase in activity with EF5 were found for any residue changes at positions 107,224 or 225 . Beneficial mutations were seen at residues involved in both predicted modes of binding.

At four sites (40,43, 103 and 215) only two possible residue changes were found to confer improvement, and each of these resulted in a less than two-fold increase in the enzyme's ability to sensitise E. coli to either imaging agent (as determined by the fold decrease in 2-NI $\mathrm{IC}_{50}$ for the mutant NTR compared to NfsA_Ec). Many of these mutations were relatively conservative; both the L103V and S40T mutations only removed or added a methyl group to the amino acid residue respectively, at site 43 both favourable changes were to acidic residues, and at residue 215 mutation of the basic His residue was only found to be favourable if substituted by either of the two other basic residues, Lys or Arg. 


\begin{tabular}{|c|c|c|c|c|c|c|}
\hline NTR & $\begin{array}{l}\text { Original } \\
\text { amino acid }\end{array}$ & $\begin{array}{c}\text { Mutant } \\
\text { amino acid }\end{array}$ & $\begin{array}{c}\mathrm{EF5}^{\mathrm{IC}} \mathrm{C}_{50} \\
(\mu \mathrm{M})\end{array}$ & $\begin{array}{l}\text { Fold increase in } \\
\text { EF5 sensitisation }{ }^{1}\end{array}$ & $\begin{array}{c}\text { Fmiso IC } C_{50} \\
(\mu \mathrm{M})\end{array}$ & $\begin{array}{c}\text { Fold increase in } \\
\text { F-miso sensitisation }\end{array}$ \\
\hline NfsA_Ec & & & $127 \pm 2$ & - & & - \\
\hline S40T & Ser & Thr & $81 \pm 4$ & 1.6 & $58 \pm 6$ & 1.7 \\
\hline$S 40 W^{2}$ & & Trp & $88 \pm 6$ & 1.4 & $53 \pm 3$ & 1.8 \\
\hline S41M ${ }^{2}$ & Ser & Met & $59 \pm 4$ & 2.1 & $34 \pm 5$ & 2.9 \\
\hline S41Q & & Gln & $62 \pm 6$ & 2.1 & $37 \pm 2$ & 2.6 \\
\hline$S 41 Y^{2}$ & & Tyr & $67 \pm 6$ & 1.9 & $38 \pm 4$ & 2.6 \\
\hline$S 41 C^{2}$ & & Cys & $68 \pm 2$ & 1.9 & $37 \pm 2$ & 2.6 \\
\hline S41G & & Gly & $71 \pm 6$ & 1.8 & $60 \pm 5$ & 1.6 \\
\hline$F 42 N^{2}$ & Phe & Asn & $58 \pm 4$ & 2.1 & $50 \pm 9$ & 2.0 \\
\hline$F 42 A^{2}$ & & Ala & $62 \pm 2$ & 2.0 & $62 \pm 5$ & 1.6 \\
\hline$F 421^{2}$ & & Ile & $67 \pm 0$ & 1.9 & $44 \pm 3$ & 2.2 \\
\hline $\mathrm{F} 42 \mathrm{H}$ & & His & $69 \pm 3$ & 1.8 & $59 \pm 3$ & 1.6 \\
\hline F42W & & Trp & $71 \pm 4$ & 1.8 & $49 \pm 3$ & 2.0 \\
\hline F42R & & Arg & $82 \pm 2$ & 1.5 & $47 \pm 2$ & 2.0 \\
\hline $\mathrm{L} \mathrm{CD}^{2}$ & Leu & Asp & $91 \pm 5$ & 1.4 & $74 \pm 5$ & 1.3 \\
\hline L43E & & Glu & $100 \pm 11$ & 1.3 & $86 \pm 7$ & 1.1 \\
\hline C45H & Cys & His & $57 \pm 2$ & 2.2 & $87 \pm 4$ & 1.1 \\
\hline$C 45 M^{2}$ & & Met & $58 \pm 1$ & 2.2 & $48 \pm 5$ & 2.0 \\
\hline $\mathrm{C} 45 \mathrm{E}^{2}$ & & Glu & $59 \pm 5$ & 2.2 & $57 \pm 4$ & 1.7 \\
\hline $\mathrm{C} 4 \mathrm{~L}^{2}$ & & Leu & $65 \pm 4$ & 2.0 & $56 \pm 6$ & 1.7 \\
\hline C45D & & Asp & $74 \pm 2$ & 1.7 & $89 \pm 8$ & 1.1 \\
\hline$F 83 A^{2}$ & Phe & Ala & $73 \pm 6$ & 1.7 & $73 \pm 7$ & 1.3 \\
\hline F83D & & Asp & $73 \pm 3$ & 1.7 & $46 \pm 2$ & 2.1 \\
\hline F83R & & Arg & $76 \pm 2$ & 1.7 & $68 \pm 5$ & 1.4 \\
\hline F83G & & Gly & $81 \pm 3$ & 1.6 & $55 \pm 6$ & 1.8 \\
\hline L103V $^{2}$ & Leu & Val & $74 \pm 2$ & 1.7 & $73 \pm 6$ & 1.3 \\
\hline $\mathrm{L}^{103 Q^{2}}$ & & Gln & $80 \pm 4$ & 1.6 & $85 \pm 4$ & 1.1 \\
\hline$N 134 R^{2}$ & Asn & Arg & $69 \pm 4$ & 1.8 & $51 \pm 2$ & 1.9 \\
\hline N134A & & Ala & $75 \pm 2$ & 1.7 & $42 \pm 3$ & 2.3 \\
\hline N134W & & Trp & $81 \pm 3$ & 1.6 & $117 \pm 7$ & 0.8 \\
\hline$H 215 R^{2}$ & His & Arg & $90 \pm 3$ & 1.4 & $64 \pm 3$ & 1.5 \\
\hline H215K & & Lys & $96 \pm 3$ & 1.3 & $62 \pm 4$ & 1.6 \\
\hline
\end{tabular}

Table 5-1 NfsA_Ec single mutants showing increased EF5 and F-miso metabolism on overexpression in $\boldsymbol{E}$. coli SOS-R2. Drug dependent growth inhibition was monitored by measuring strain turbidity $\left(\mathrm{OD}_{600}\right)$ pre and post four hour incubation with a two-fold dilution series of drug. Percentage growth relative to unchallenged controls was determined and SigmaPlot ${ }^{\mathrm{TM}} 10.0$ used to calculate the $\mathrm{IC}_{50}$. Data are the average of at least three independent experiments $\pm \mathrm{SEM}$. Displayed are the amino acid residue changes found in improved mutants, the $\mathrm{IC}_{50}$ values for each NTR over-expression strain on drug exposure and the fold increase in drug sensitisation compared to NfsA_Ec wild type. 
At other sites a greater number of residues were found to improve activity, ranging from three beneficial mutations at site 134 to six at site 42 . At each of these sites, at least one mutation was identified that conferred a > two-fold increase in the enzyme's ability to sensitise $E$. coli to at least one of the imaging agents. The largest effect was seen with changing Ser to Met at site 41, which conferred a 2.9 fold increase in F-miso sensitisation. At these sites the beneficial mutations tended to span a wide range of amino acid sidechain properties, for example replacing the polar Cys45 residue with either basic (His), acidic (Glu, Asp) or hydrophobic (Met, Leu) residues could improve activity. That there is so much variation in these beneficial mutations suggests that at these sites the wild type residue may be actively inhibiting 2-NI metabolism.

\subsubsection{Combination of single mutations to generate NfsA_Ec variants further improved in 2-NI metabolism}

To further improve metabolism of 2-NI imaging agents by NfsA_Ec, individual beneficial mutations were combined to create a library of multi-site mutants. To achieve this, a gene library was created where at each mutation site the codon contained degenerate bases such that it could designate only the desired mutant residue or the original wild type residue. By screening such a library all possible combinations of multiple mutations can be sampled. The degenerate base code and theoretical examples of its design are presented in Table 5-2.

\begin{tabular}{|c|c|c|c|c|c|c|c|c|c|c|c|}
\hline \multicolumn{12}{|c|}{ IUB Degenerate Bases Code Table } \\
\hline Code & $\mathrm{N}$ & V & B & $\mathrm{H}$ & $\mathrm{D}$ & $\mathrm{K}$ & $\mathrm{S}$ & W & $\mathrm{M}$ & $\mathrm{Y}$ & $\mathrm{R}$ \\
\hline Bases & $\mathrm{A}, \mathrm{C}, \mathrm{G}, \mathrm{T}$ & $\mathrm{G}, \mathrm{A}, \mathrm{C}$ & $\mathrm{G}, \mathrm{T}, \mathrm{C}$ & $\mathrm{A}, \mathrm{T}, \mathrm{C}$ & $\mathrm{G}, \mathrm{A}, \mathrm{T}$ & $\mathrm{G}, \mathrm{T}$ & $\mathrm{G}, \mathrm{C}$ & $\mathrm{A}, \mathrm{T}$ & $A, C$ & $\mathrm{C}, \mathrm{T}$ & $A, G$ \\
\hline & e.g & $\underline{\text { SSG }}$ & Codon & Amino acid & & CRT & Codon & Amino acid & & & \\
\hline & & & GGG & Gly & & & CAT & His & & & \\
\hline & & & CGG & Arg & & & CGT & Arg & & & \\
\hline & & & GCG & Ala & & & & & & & \\
\hline & & & CCG & Pro & & & & & & & \\
\hline
\end{tabular}

Table 5-2 Table showing IUB degenerate base code. Two examples of amino acids specified by degenerate codons included below.

Ideally any degenerate codons used would be restrictive enough to code only for either the wild type or the desired beneficial mutant residue(s), since if any additional residues are also "allowed" by the codon it unnecessarily increases the library size. Therefore mutants chosen for combination were selected after considering not only the 
improvements in 2-NI metabolism they conferred, but also whether codon restriction was possible. For example while at Ser41 the Met mutation conferred the greatest improvement in 2-NI metabolism, no redundancy codon for incorporating only Ser and Met is possible; so tested at this site instead were the next most active Cys and Tyr substitutions. For a number of sites (Ser40, Phe42, Leu103, His215) the most active or near most active substitution could be incorporated with appropriate redundancy codons. At remaining sites (Leu43, Cys45, Phe83, Asn134) no redundancy codon for incorporating only the wild type residue or any beneficial mutant residue existed. However using a slightly modified strategy mutations at these sites could be included in the library. This was done by designing two separate forward primers for amplification of the gene fragments containing these sites, one primer specifying the desired mutant and one primer specifying the original sequence, and including both resulting fragments in a 1:1 ratio when generating the full length gene using overlap PCR. Due to the high activity observed with the substitutions N134A and C45L/M (noting that Met and Leu can be simultaneously encoded for by one redundancy codon) these mutations were included in the library. All possible degeneracy codons can be seen in Appendix 2.

In summary ten mutations over eight sites were chosen: S40T, S41C, S41Y, F42I, C45L C45M, L103V, N134A and H215R and a final mutation R12S (found in separate prodrug focused evolution work by Dr. Janine Copp, and shown by me to confer 1.6 and 1.7 fold increases in EF5 and F-miso sensitisation - results not shown). The ten mutations were able to be combined in 576 possible ways.

\subsubsection{Further assessment of selected beneficial single mutations}

Prior to the construction of the multi-site mutant library the ten selected single mutations were further assessed, both by SDS-PAGE visualisation of their relative expression levels in E. coli SOS-R2 and by determination of their apparent kinetic parameters with EF5, carried out using purified His6-tagged recombinant proteins.

\subsubsection{SDS-PAGE of relative protein expression}

Protein expression levels of the mutant variants relative to NfsA_Ec were assessed by SDS-PAGE (Figure 5-2). Relative expression of the clones was measured using ImageJ. Both NfsA_Ec cultures showed similar expression levels (within 10\% of each other) and no single mutant cultures showed greater NTR expression than both controls. The only 
mutants showing notably different expression were S41C and L103V, at 60-70\% of wild type NfsA_Ec levels.

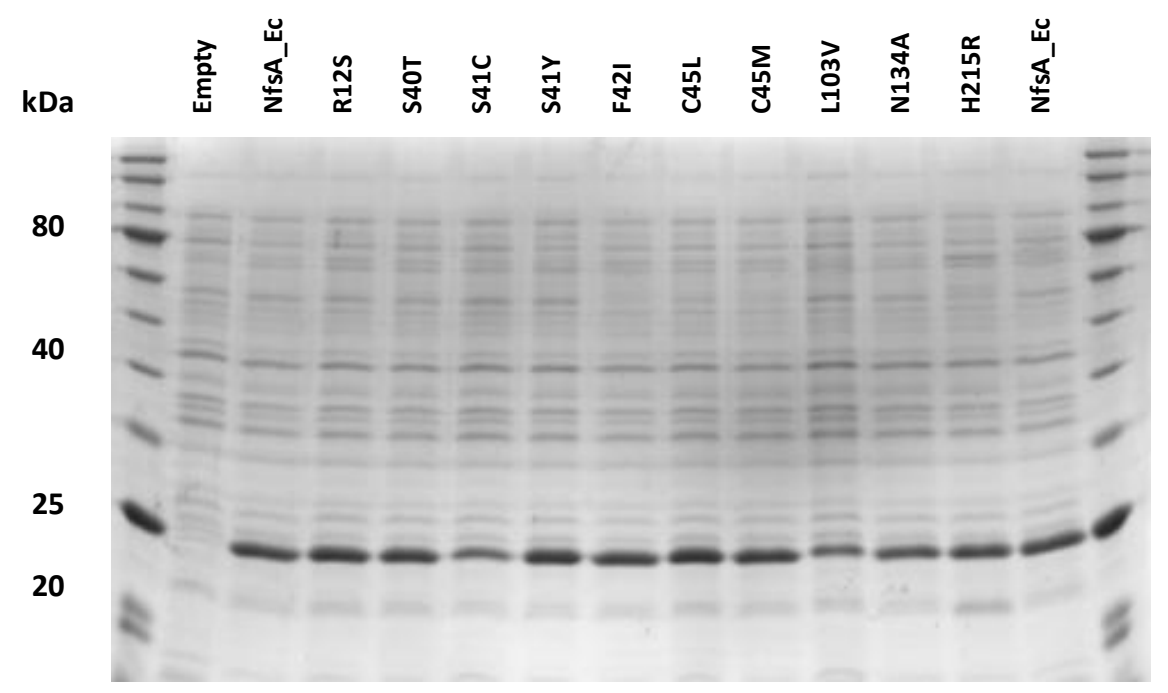

Figure 5-2 SDS-PAGE gel showing NfsA_Ec single mutant relative protein expression levels from SOS-R2 pUXC::ntr constructs. NTR over-expression was induced with $50 \mu \mathrm{M}$ IPTG for 6.5 hours, as per $\mathrm{IC}_{50}$ assay protocol, prior to sample collection. Samples were normalised for cell density $\left(\mathrm{OD}_{600}\right)$ and $10 \mu \mathrm{L}$ of sample loaded per lane.

\subsubsection{Reduction of EF5 by His6-tagged purified proteins}

To further investigate the effect of each single mutation on the reduction of EF5 by NfsA_Ec the apparent kinetic parameters of the reaction were determined. All 10 NfsA_Ec single mutant genes were cloned into the plasmid pET-28(a+), each pET:ntr construct was individually transformed into the E. coli strain BL:21, and NTR overexpression was induced by IPTG in LBK media containing betaine and sorbitol at $18{ }^{\circ} \mathrm{C}$ for 40 hours. Eight of the ten mutants were expressed in sufficient soluble quantities to allow purification by nickel affinity chromotography, however F42I and H215R were found predominantly in the insoluble fraction and as such purification of these variants was not successful. The NfsA_Ec wild type protein was prepared and purified in parallel with the mutants.

The apparent kinetic parameters for the reduction of EF5 by NfsA_Ec wild type and mutant proteins can be seen in Figure 5-3. The activity of NfsA_Ec was very similar to previous measurements (Table 3-1) Of the mutants only two variants, R12S and N134A, showed increased EF5 metabolism as measured by the specificity constant $k_{\text {cal }} / K_{m}$, while two further proteins S40T and S41C showed roughly the same activity as NfsA_Ec (52 $\mathrm{mM}^{-1} \mathrm{~s}^{-1}$ and $53 \mathrm{mM}^{-1} \mathrm{~s}^{-1}$ versus $56 \mathrm{mM}^{-1} \mathrm{~s}^{-1}$ respectively). The other four tested mutants 
all showed decreased activity by this measure. For three of the mutants (S41Y, C45L and $\mathrm{C} 45 \mathrm{M})$ this was due to a decrease in apparent $k_{\text {cat }}$. Overall six of the eight mutants tested showed a decrease in apparent $k_{c a t}$, however for all mutants in which this occurred the apparent $K_{m}$ was also decreased.
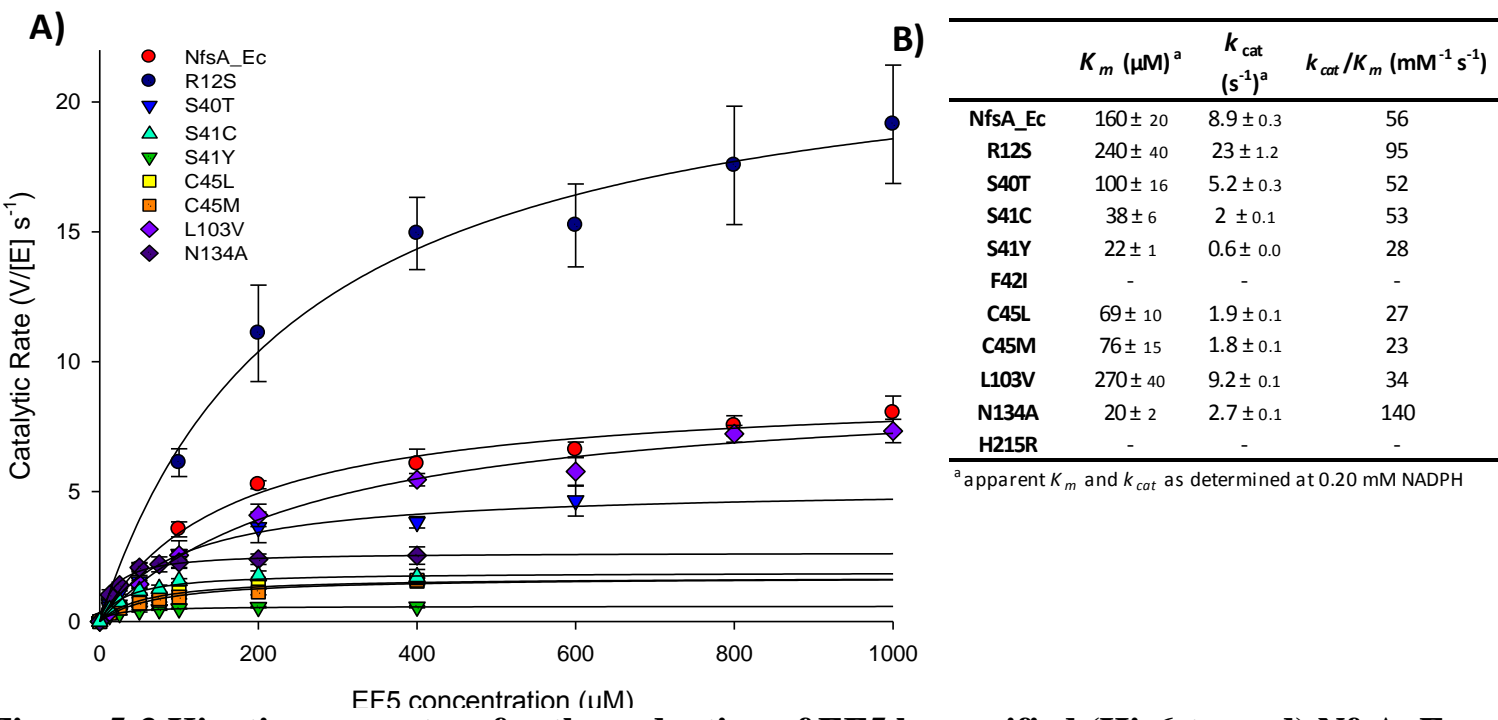

Figure 5-3 Kinetic parameters for the reduction of EF5 by purified (His6-tagged) NfsA_Ec single mutants A) Plots of initial rates of reaction/enzyme concentration with varying concentrations of EF5 and $0.2 \mathrm{mM}$ NADPH. At each EF5 concentration rates were measured in at least triplicate and errors represent \pm 1 standard deviation. B) Apparent $K_{m}$ and $k_{\text {cat }}$ at $0.2 \mathrm{mM}$ NADPH derived using Sigmaplot 10.0 (Systat Software Inc., Richmond, CA). Reaction rate was monitored at $340 \mathrm{~nm}$ and an extinction coefficient of $19,000 \mathrm{M}^{-1} \mathrm{~cm}^{-1}$ used see section 2.11.1.

\subsubsection{Construction of a multi-site mutant NfsA_Ec library}

A mix of full length NfsA_Ec genes comprising all possible combinations of the ten selected single mutations was generated via overlap PCR of five fragments of the NfsA_Ec gene. These fragments had themselves been PCR amplified from a wild type NfsA_Ec gene template using primers with the specifically designed degeneracy codons to introduce desired mutations (Figure 5-4).

This gene library was ligated into pUCX, transformed into SOS-R2 and 10 random clones were selected and sequenced to confirm correct assembly (See Appendix 3 for sequences). All ten individual mutations were represented within the 10 selected clones. PCR screening of 20 colonies revealed that 17 had inserts, enabling the insert ratio to be estimated at $85 \%$; based on this, to allow $95 \%$ coverage of the library 2024 colonies were picked into 96 well micro-plates that also contained empty plasmid, NfsA_Ec wild type and S41Y controls on each plate. 
GGGCATATGACGCCAACCATTGAACTTATTTGTGGCCATMGCTCCATTCGCCATTTCACTGA TGAACCCATTTCCGAAGCGCAGCGTGAGGCGATTATTAACAGCGCCCGTGCGACGTCCAGTW CTTVTWTTTTGCAGTGC/WTGAGTAGCATTATTCGCATTACCGACAAAGCGTTACGTGAAGA ACTGGTGACGCTGACCGGCGGGCAAAAACACGTAGCGCAAGCGGCGGAGTTCTGGGTGTTCT GTGCCGACTTTAACCGCCATTTACAGATCTGTCCGGATGCTCAGCTCGGCCTGGCGGAACAA CTGTTGSTGGGTGTCGTTGATACGGCAATGATGGCGCAGAATGCATTAATCGCAGCGGAATC GCTGGGATTGGGCGGGGTATATATCGGCGGCCTGCGCAAT/CGCAATATTGAAGCGGTGACG AAACTGCTTAAATTACCGCAGCATGTTCTGCCGCTGTTTGGGCTGTGCCTTGGCTGGCCTGC GGATAATCCGGATCTTAAGCCGCGTTTACCGGCCTCCATTTTGGTGCATGAAAACAGCTATC AACCGCTGGATAAAGGCGCACTGGCGCAGTATGACGAGCAACTGGCGGAATATTACCTCACC CGTGGCAGCAATAATCGCCGGGATACCTGGAGCGATCRTATCCGCCGAACAATCATTAAAGA AAGCCGCCCATTTATTCTGGATTATTTGCACAAACAGGGTTGGGCGACGCGCTAAGTCGACG GGG

Figure 5-4 Representation of a multi-site mutant NfsA_Ec gene library for testing of all 576 possible combinations of ten single mutations found to improve NfsA_Ec metabolism of EF5 and F-miso- R12S, S40T, S41C/Y, F42I, C45L/M, L103V, N134A and H215R. Constructed by combining five fragments (which contained the various mutations) using overlap PCR. Regions of the gene which corresponded to the forward primers used to amplify the individual fragments are displayed as blue, the reverse primers displayed as red and where these sequences overlap displayed as purple. Mutant codons used are displayed as green. For introducing mutations at site 45 and site 134, where two different primers be used to separately amplify gene fragments which were later mixed, the codon difference in each primer is denoted by a forward slash symbol (/). Restriction sites for cloning are underlined.

\subsubsection{Screening of a multi-site mutant NfsA_Ec library for variants with improved 2-NI imaging agent metabolism}

RB6145 SOS assays were not used for screening of the multi-site library. We thought it possible that the increased specialization of the active site due to multiple mutations could cause a larger differentiation between activities with the three compounds. Additionally, as single mutants were already more sensitised to EF5 and F-miso than wild type NfsA_Ec, growth inhibition screens could be conducted using lower concentrations of imaging agent than would have been previously possible.

Each plate was screened with one growth inhibition assay in which duplicate cultures were exposed to either EF5 $(60 \mu \mathrm{M})$ or F-miso $(45 \mu \mathrm{M})$. Clones exhibiting equal or greater drug dependent growth inhibition compared to the single mutant S41Y were retested by a second growth inhibition assay before hits were confirmed via determination of drug $\mathrm{IC}_{50}$ values (Figure 5-5). The 12 clones that showed a $>3.5$ fold increase in drug sensitisation for at least one of the compounds were sequenced, and collectively found to comprise a total of eight different sequences (Table 5-3). 
A)

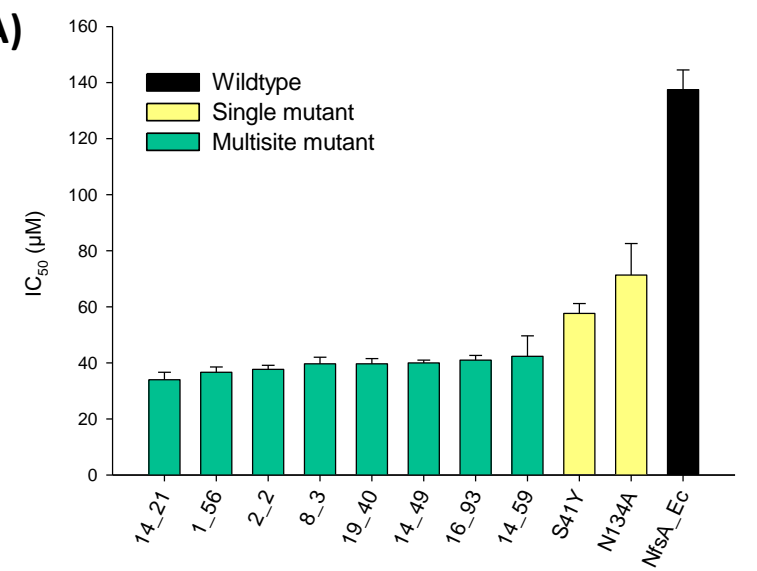

B)

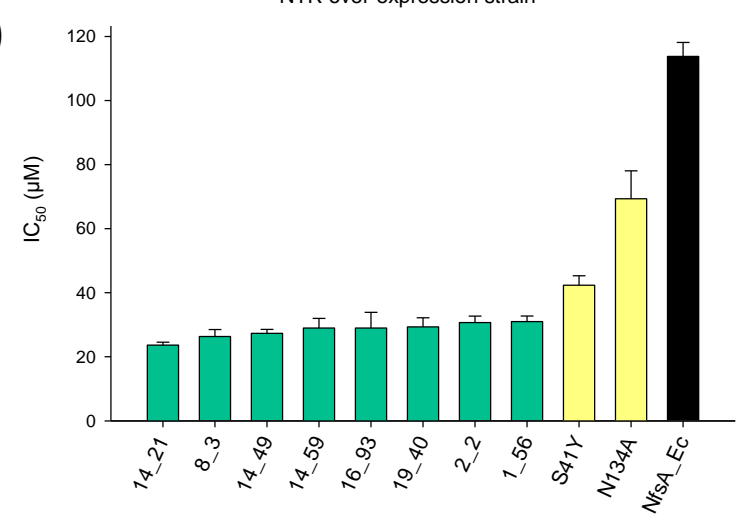

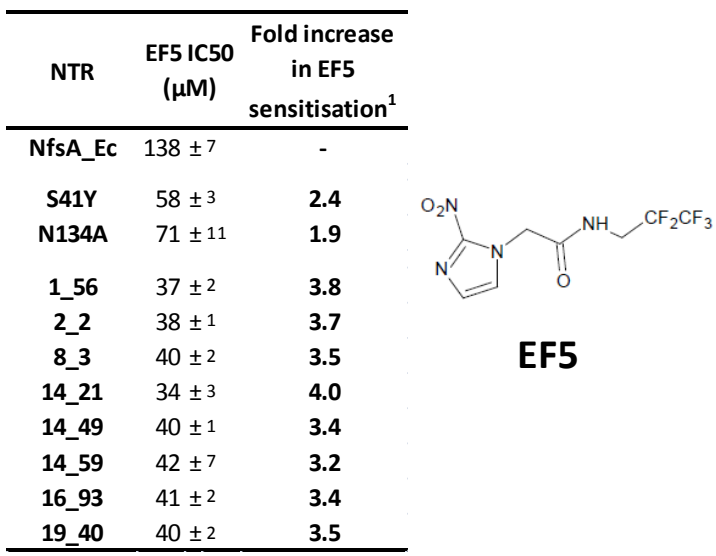

\begin{tabular}{ccc}
\hline NTR & $\begin{array}{c}\text { F-miso } \\
\text { IC50 }(\mu \mathrm{M})\end{array}$ & $\begin{array}{c}\text { Fold increase } \\
\text { in F-miso } \\
\text { sensitisation }\end{array}$ \\
\hline NfsA_Ec & $114 \pm 4$ & - \\
S41Y & $42 \pm 3$ & $\mathbf{2 . 7}$ \\
N134A & $69 \pm 9$ & $\mathbf{1 . 6}$ \\
1_56 & $31 \pm 2$ & $\mathbf{3 . 7}$ \\
2_2 & $31 \pm 2$ & $\mathbf{3 . 7}$ \\
8_3 & $26 \pm 2$ & $\mathbf{4 . 3}$ \\
14_21 & $24 \pm 1$ & $\mathbf{4 . 8}$ \\
14_49 & $27 \pm 1$ & $\mathbf{4 . 2}$ \\
14_59 & $29 \pm 3$ & $\mathbf{3 . 9}$ \\
16_93 & $29 \pm 5$ & $\mathbf{3 . 9}$ \\
19_40 & $29 \pm 3$ & $\mathbf{3 . 9}$ \\
\hline
\end{tabular}

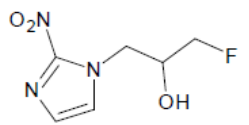

F-miso

NTR over-expression strain

${ }^{1}$ Derived by dividing 150 value on NfsA_Ec expression by the 56 Geen on expression of the improved mutant

Figure 5-5 A) EF5 and B) F-miso IC $_{50}$ values for SOS-R2 E. coli strains over-expressing NfsA_Ec wild type or mutants evolved for improved 2-NI metabolism. Drug dependent growth inhibition was monitored and SigmaPlot ${ }^{\mathrm{TM}} 10.0$ used to calculate the $\mathrm{IC}_{50}$. Data are the average of at least three independent experiments \pm SEM. $\mathrm{IC}_{50}$ values for the single site mutants S41Y and N134A were determined as controls. Multi-site mutants are referred to by original plate and well position in the mutant library. Graphed strains are ordered by increasing $\mathrm{IC}_{50}$.

\begin{tabular}{|c|c|c|c|c|c|c|c|c|c|c|c|}
\hline Mutants & Sequence & R12S & S40T & S41C & S41Y & $\mathrm{F} 42 \mathrm{I}$ & C45L & C45M & L103V & N134A & H215R \\
\hline \#1_56* & R12S/S40T/S41Y/F42I & $\checkmark$ & $\checkmark$ & & $\checkmark$ & $\checkmark$ & & & & & \\
\hline \#2_2 & R12S/S40T/S41Y/F42I/N134A & $\checkmark$ & $\checkmark$ & & $\checkmark$ & $\checkmark$ & & & & $\checkmark$ & \\
\hline \#8_3* & S40T/S41Y/L103V/N134A & & $\checkmark$ & & $\checkmark$ & & & & $\checkmark$ & $\checkmark$ & \\
\hline \# 12_9* & S40T/S41Y/N134A & & $\checkmark$ & & $\checkmark$ & & & & & $\checkmark$ & \\
\hline \# 14_21* & S41C/L103V/N134A & & & $\checkmark$ & & & & & $\checkmark$ & $\checkmark$ & \\
\hline \# 14_49 & S41Y/L103V/N134A & & & & $\checkmark$ & & & & $\checkmark$ & $\checkmark$ & \\
\hline \# 14_59 & S40T/S41C/L103V/N134A & & $\checkmark$ & $\checkmark$ & & & & & $\checkmark$ & $\checkmark$ & \\
\hline \# 16_93 & S41Y/F42I/L103V/N134A & & & & $\checkmark$ & $\checkmark$ & & & $\checkmark$ & $\checkmark$ & \\
\hline
\end{tabular}

* identical mutant found twice in improved clones

Table 5-3 Amino acid residue changes found in NfsA_Ec mutants exhibiting improved EF5 and F-miso metabolism as determined in $\mathrm{IC}_{50}$ assays presented in Figure 5-5. 
There were strong patterns in the mutations found in the improved clones. None of the sequences contained changes at the 45 or 215 position. In contrast 7 of the 8 improved variants contained the N134A mutation and all were mutated at position 41 with changes to Tyr (6/8) favoured over Cys. For five out of the eight sequences the crucial mutations appear to be S41X/L103V/N134A ("X" being either Tyr or Cys). There is no notable difference between 14_49 (S41Y/L103V/N134A) and 8_3 (14_49 + S40T) or 16_93 $\left(14 \_49+\right.$ F42I $)$ and also between 14_21 (S41C/L103V/N134A) and 14_59 (14_21 + S40T).

Three clones were selected for further study; these were 14_21 and14_49, based on the predominance of the S41X/L103V/N134A mutations; and clone 1_56, chosen to expand the diversity of the mutations examined.

\subsubsection{Further assessment of selected multi-site NfsA_Ec mutants}

\subsubsection{SDS-PAGE of relative protein expression}

Protein expression levels of the mutant variants relative to NfsA_Ec were assessed by SDS-PAGE (Figure 5-6). Both 14_21 and 14_49 expressed at identical levels to wild type NfsA_Ec, whereas 1_56 expression was slightly decreased (measured by densitometry at $\sim 75 \%$ of wild type NfsA_Ec, using ImageJ).

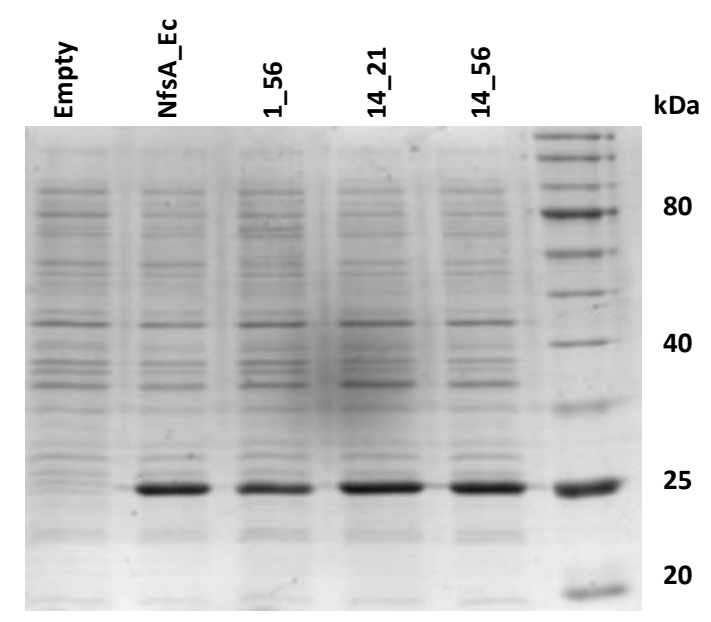

Figure 5-6 SDS-PAGE gel showing NfsA_Ec relative protein expression levels of multi-site mutants, expressed from SOS-R2 pUXC:ntr constructs. NTR over-expression was induced with $50 \mu \mathrm{M}$ IPTG for 6.5 hours, as per $\mathrm{IC}_{50}$ assay protocol, prior to sample collection. Samples were normalised for cell density $\left(\mathrm{OD}_{600}\right)$ and $15 \mu \mathrm{L}$ of sample loaded per lane. 


\subsubsection{Reduction of EF5 by His6-tagged purified proteins}

The three NfsA_Ec multi site mutants selected for further study were purified as His6tagged proteins as previously described, however 1_56 was found predominantly in the insoluble fraction and as such purification was not successful. Apparent kinetic parameters with EF5 were determined for 14_21 and 14_49. As can be seen in Table 5-4 the trend of in vivo high activity enzymes showing substantially lower apparent $k_{\text {cat }}$ but also substantially lower $K_{m}$ values was again apparent.

\begin{tabular}{cccc}
\hline & $\boldsymbol{K}_{\boldsymbol{m}}(\boldsymbol{\mu M})^{\mathrm{a}}$ & $\begin{array}{c}\boldsymbol{k}_{\text {cat }} \\
\left(\mathbf{s}^{-1}\right)^{\mathrm{a}}\end{array}$ & $\begin{array}{c}\boldsymbol{k}_{\text {cat }} / \boldsymbol{K}_{\boldsymbol{m}} \\
\left(\mathrm{mM}^{-\mathbf{1}} \mathbf{s}^{-\mathbf{1}}\right)\end{array}$ \\
\hline $\begin{array}{c}\text { NfsA_Ec } \\
\text { Single mutants: }\end{array}$ & $160 \pm 20$ & $8.9 \pm 0.3$ & 56 \\
S41Y & $22 \pm 1.1$ & $0.6 \pm 0.01$ & 28 \\
C45L & $69 \pm 10$ & $1.9 \pm 0.1$ & 27 \\
C45M & $76 \pm 15$ & $1.8 \pm 0.1$ & 23 \\
Multi mutants: & & & \\
14_21 & $6.5 \pm 1.8$ & $0.23 \pm 0.01$ & 36 \\
14_49 & $12 \pm 2.3$ & $0.19 \pm 0.01$ & 16 \\
\hline
\end{tabular}

a apparent $K_{m}$ and $k_{\text {cat }}$ as determined at $0.20 \mathrm{mM} \mathrm{NADPH}$

Table 5-4 Kinetic parameters for the reduction of EF5 by purified (His6-tagged) NfsA_Ec multi-site mutants. Rates of reduction at varying concentrations of EF5 and a fixed concentration of excess NADPH $(0.20 \mathrm{mM})$ were monitored at $340 \mathrm{~nm}$, rates measured in quadruplicate. Apparent $K_{m}$ and $k_{\text {cat }}$ derived using Sigmaplot 10.0 (Systat Software Inc., Richmond, CA). Extinction coefficient used was $19,000 \mathrm{M}^{-1} \mathrm{~cm}^{-1}$; for explanation of determination see section 2.11.1 . Previously measured apparent kinetic parameters of NfsA_Ec wild type and three single mutants are shown for comparison.

\subsubsection{Activity with the nitroaromatic prodrug PR104-A and the 5- NI antibiotic metronidazole}

To assess the effect these mutations had on the ability to metabolise prodrugs, E. coli SOS-R2 strains expressing the mutants S41Y, 1_56, 14_21 or 14_49 were exposed to PR$104 \mathrm{~A}$, and $\mathrm{IC}_{50}$ values for each strain determined (Figure 5-7A). In addition, each mutant's activity with metronidazole, a 5-NI compound which is a known NTR substrate, was tested (Figure 5-7 B). Pleasingly none of the multi-site mutants had lost any ability to sensitise E. coli to PR-104A metabolism. It should be noted though that the single mutant S41Y showed the greatest (1.9 fold) increase in sensitisation, suggesting that the other individual residue changes in 1_56 and 14_49 (which also contain the S41Y mutation) may somewhat reduce activity with PR-104A. In contrast all of the multi-site 
mutants showed decreased metabolism of metronidazole compared to NfsA_Ec WT and S41Y.

A)

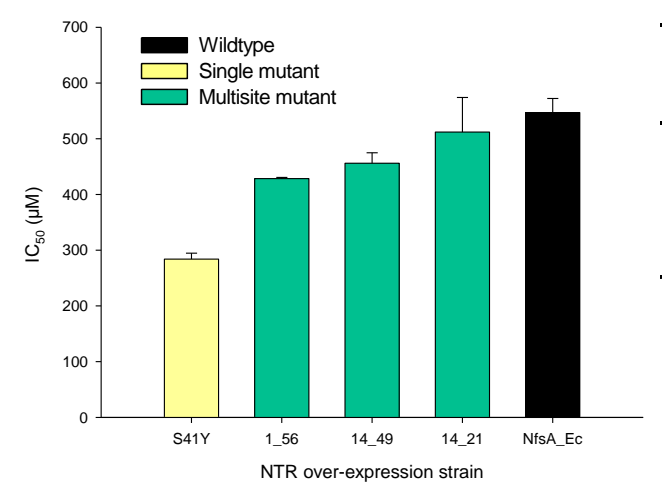

\begin{tabular}{lcc}
\hline \multicolumn{1}{c}{ NTR } & $\begin{array}{c}\text { PR-104A } \\
\text { IC50 } \\
(\boldsymbol{\mu M})\end{array}$ & $\begin{array}{c}\text { Fold increase } \\
\text { in PR-104A } \\
\text { sensitisation }\end{array}$ \\
\hline NfsA_Ec & $547 \pm 25$ & - \\
S41Y & $284 \pm 11$ & 1.9 \\
1_56 & $429 \pm 2$ & 1.3 \\
14_21 & $512 \pm 62$ & 1.1 \\
14_49 & $456 \pm 19$ & 1.2 \\
\hline
\end{tabular}

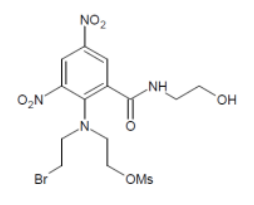

PR-104A

B)

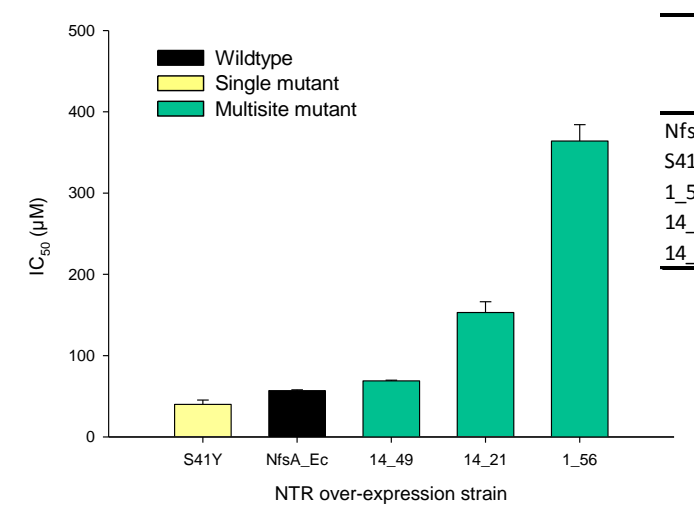

\begin{tabular}{lcc}
\hline \multicolumn{1}{c}{ NTR } & $\begin{array}{c}\text { Metro } \\
\text { IC }_{50} \\
(\boldsymbol{\mu} \text { M) })\end{array}$ & $\begin{array}{c}\text { Fold increase } \\
\text { in Metro } \\
\text { sensitisation }\end{array}$ \\
\hline NfsA_Ec & $57 \pm 1$ & - \\
S41Y & $40 \pm 5$ & 1.4 \\
$1 \_56$ & $364 \pm 20$ & 0.15 \\
14 21 & $153 \pm 13$ & 0.37 \\
$14 \_49$ & $69 \pm 2$ & 0.83 \\
\hline
\end{tabular}

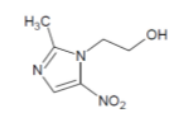

Metro

${ }^{1}$ Derived by dividing IC 50 value on $\mathrm{NfSA}$ _Ec expression by the IC 50 seen on expression of the improved mutant

Figure 5-7 PR-104A and metronidazole (Metro) $\mathrm{IC}_{50}$ values for SOS-R2 E. coli strains overexpressing NfsA_Ec wild type or mutants evolved for improved 2-NI metabolism. Drug dependent growth inhibition was monitored as previously described and SigmaPlot ${ }^{\mathrm{TM}} 10.0$ used to calculate the $\mathrm{IC}_{50}$. Data are the average of three independent experiments \pm SEM. Graphed strains are ordered by increasing $\mathrm{IC}_{50}$.

\subsubsection{Activity of previously identified mutants with the novel 2-NI imaging agent HX4}

During the course of the research described above our laboratory began collaborating with researchers at the University of Maastricht and the experimental 2-NI imaging agent HX4 became available to our study (background information and structure available in section 1.8.3). The ability of the NfsA_Ec mutants to sensitise E. coli to HX4 was therefore tested. Serendipitously, the ten single mutations that had been selected for combination all also improved NfsA_Ec in vivo metabolism with HX4 (Figure 5-8A). Likewise, the sequenced multi-site mutants showed further increases in HX4 metabolism (Figure 5-8B) of a comparable range (3.2-4.2 fold increase) to the improvements seen with EF5 and F-miso. 


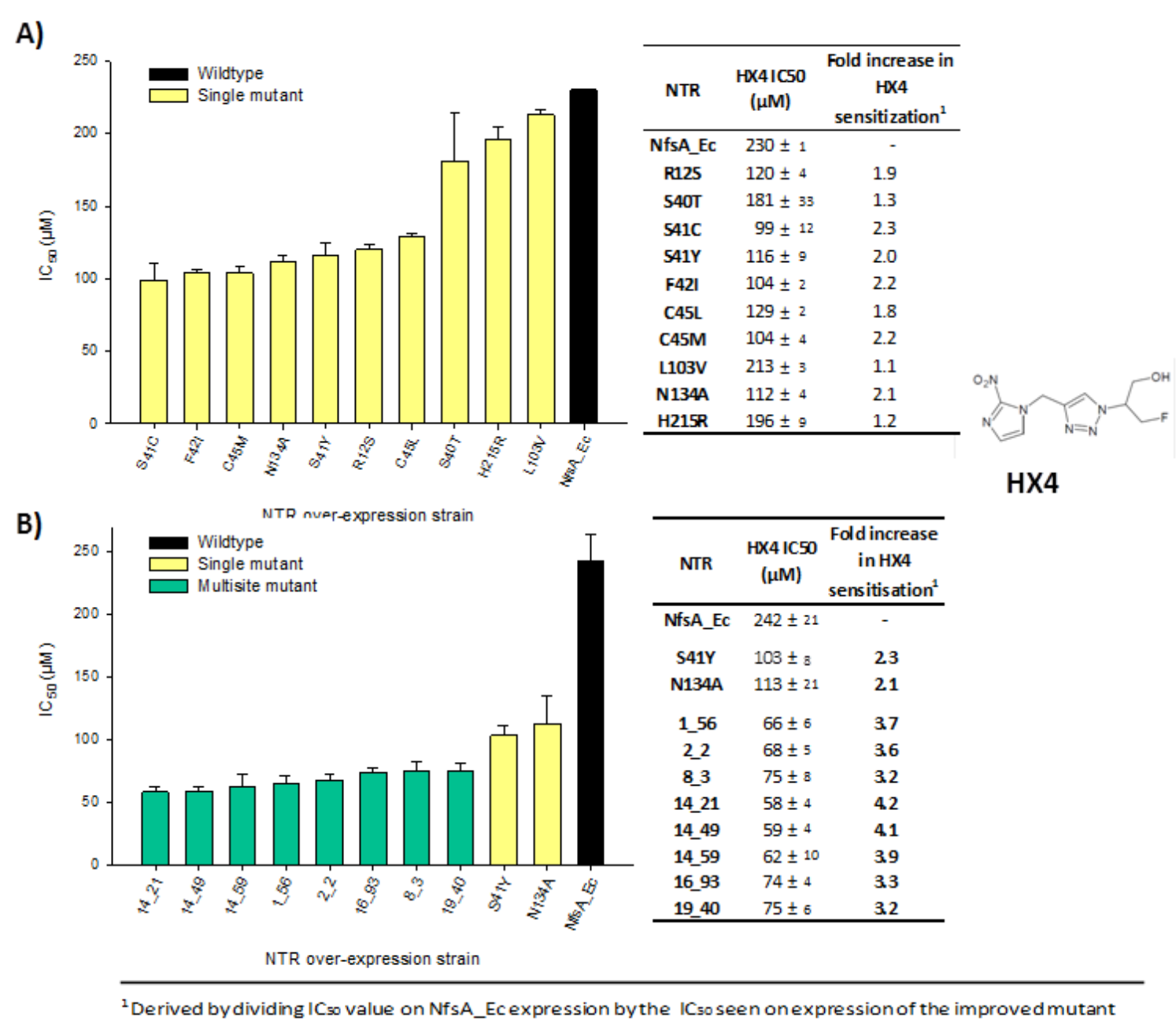

Figure 5-8 HX4 IC 50 values for SOS-R2 E. coli strains over-expressing NfsA_Ec wild type or mutants evolved for improved 2-NI metabolism. Shown are results with both single mutants (A) and multi-site mutants (B) Drug dependent growth inhibition was monitored as previously described SigmaPlot ${ }^{\mathrm{TM}} 10.0$ used to calculate the $\mathrm{IC}_{50}$. Data are the average of at least two (A) or three (B) independent experiments \pm SEM. Graphed strains are ordered by increasing $\mathrm{IC}_{50}$ value.

\subsubsection{Assessment of NfsA_Ec mutants showing improved 2-NI metabolism by expression in a human cancer cell line}

The experimental work presented in this section was performed during a short research visit to the laboratories of our collaborators at the ACSRC.

For further characterisation of the 2-NI metabolism of the NfsA_Ec variants described above, HCT-116:ntr stable cell lines were generated. Chosen for transfection of HCT-116 were the single mutant N134A and the three multi site mutants 1_56, 14_21 and 14_49. Transfer of the mutant NfsA_Ec genes into the HCT-116 expression plasmid F279-V5 was carried out using Invitrogen Gateway® technology (section 2.17.3). The F279-V5 plasmid is homebuilt by the ACSRC, and uses a CMV promoter to transcribe both the 
inserted gene sequence and a puromycin resistance sequence as a single mRNA transcript, with an internal ribosome entry site allowing for separate translation of the inserted protein and the puromycin resistance protein. To generate stable expression cell lines, HCT-116 cells were transfected with F279-V5 NTR plasmids and cells which had taken up the plasmid were selected for by multiple passage cycles in media containing escalating concentrations of puromycin.

HCT-116 cell lines stably expressing NfsA_Ec and NfsB_Ec from F279-V5 had already been generated at the ACSRC and these cell lines were made available for this work.

\subsubsection{Western blot of NfsA_Ec wild type and mutant expression from HCT-} 116

Expression of the four NfsA_Ec mutant proteins compared to NfsA_Ec wild type in HCT-116 was assessed by western blotting. The cell line transfected with mutant 1_56 showed no NfsA_Ec expression whatsoever. The other three mutants showed decreased expression relative to NfsA_Ec wild type. This decreased expression was consistently observed in two western blots run using independently collected cell samples, with one blot pictured in Figure 5-9. When previously attempting to stably express a range of wild type NTRs from HCT-116 we had observed both non-existent and variable NTR expression levels (Prosser et al., 2013) but we were not expecting to see substantial variations in expression of the mutant NTRs presented here, which each differed from NfsA_Ec wild type by fewer than five amino acid residues. While variant $1 \_56$ does not express from HCT-116 and is also unable to be purified in sufficient soluble form following over-expression from E. coli these two issues were not generally found to cooccur when examining other wild type or mutant NTRs.

It should be noted that expression issues were not limited to these four mutants, in parallel work conducted by Alex Mowday, heterogeneous expression from HCT-116 was also observed for a larger suite of 17 NfsA_Ec variants. This observation will be expanded on in the discussion of this chapter. 

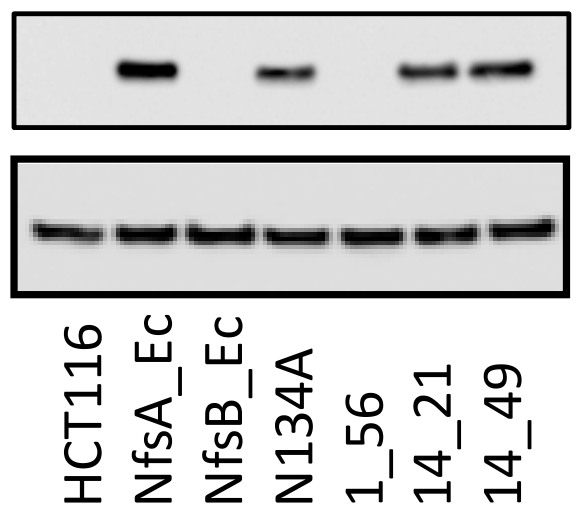

NfsA antibody

$\beta$-actin

\begin{tabular}{cc}
\hline & $\begin{array}{c}\text { Expression relative } \\
\text { to NfsA_Ec (\%) }\end{array}$ \\
\hline HCT116 & - \\
NfsA_Ec & $100 \%$ \\
NfsB_Ec & - \\
N134A & $23 \%$ \\
1_56 & - \\
14_21 & $25 \%$ \\
14_49 & $31 \%$ \\
\hline
\end{tabular}

Figure 5-9 Western blot showing expression of NfsA_Ec wild type and mutant proteins from stable HCT-116:NTR cell lines. HCT-116 cell samples were taken cells lysed and NfsA_Ec expression determined by immunoblot. $\beta$-actin loading control also shown. Relative protein expression determined by densitometry using ImageJ.

\subsubsection{Testing for functional NTR expression using NTR activated fluorogenic probes}

To test for functional expression of the mutant NfsA_Ec proteins, the cell lines were exposed to three NTR activated fluorogenic probes designed and synthesised at the ACSRC. These probes were chosen on the basis of their structural diversity (structures presented in Figure 1-10) and because N134A, 1_56, 14_21 and 14_49 were all found to metabolise these three probes at comparable levels to NfsA_Ec wild type when expressed in E. coli (results not shown).

Four weeks post the initial transfection cells were seeded into a 6 well plate and exposed to the blue fluorogenic probe FSL61. Four days later the same cell lines were seeded and exposed to the green probe FSL76 and red probe FSL178. Cells were imaged with a FLoid ${ }^{\circledR}$ Cell Imaging Station, a bench top imaging device with pre-set relief phase and blue, red and green fluorescent channels. As can be seen in Figure 5-10, while the NfsA_Ec wild type and N134A cell lines show an ability to metabolise all probes, this activity was markedly decreased in the 14_21 and 14_49 cell lines and completely absent for 1_56 (the latter being the cell line for which no NfsA_Ec expression could be detected by western blot). 
<smiles>CN(CCNC(=O)[O-])Cc1cc([N+](=O)[O-])cc2c(=O)c3ccccc3[nH]c12</smiles>

FSL76<smiles>CC(=O)Cn1ccc(=O)c2cc([N+](=O)[O-])ccc21</smiles>

FSL61

HCT116

NfsA_Ec

N134A

1_56

14_21

14_49

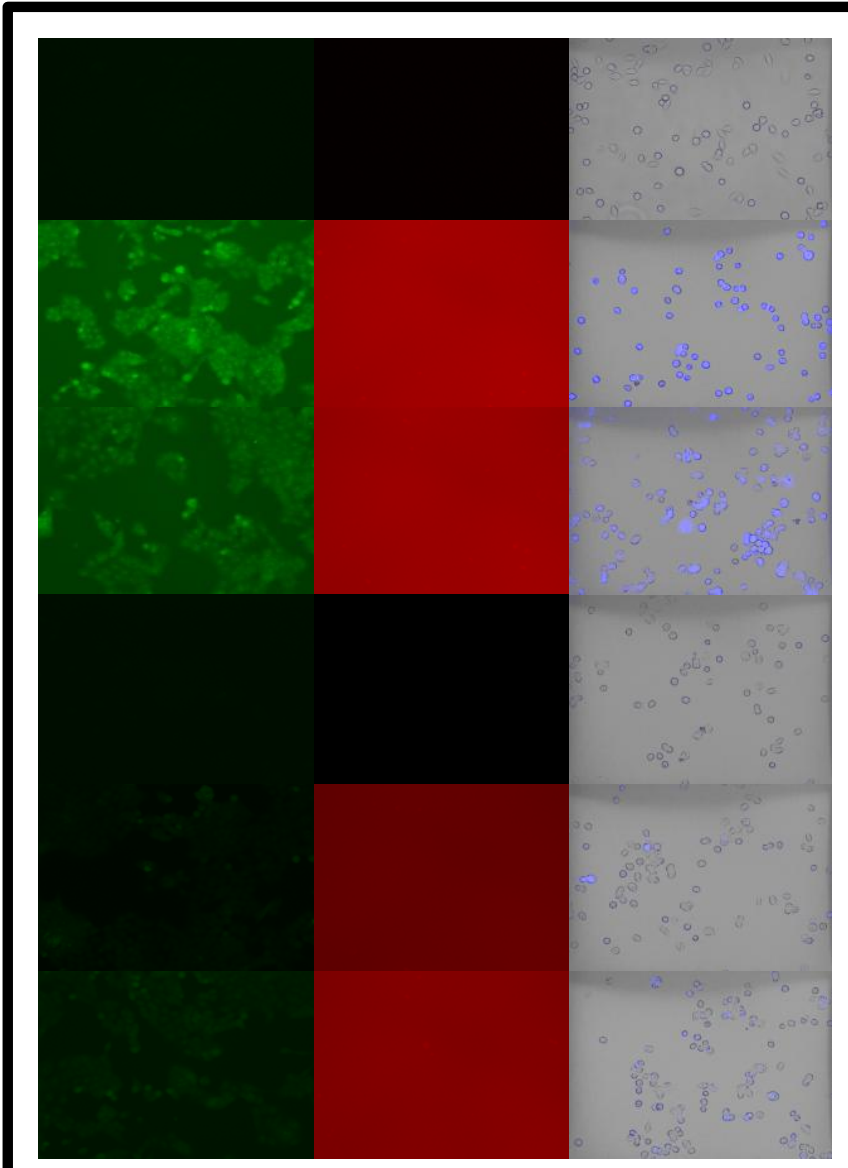

Figure 5-10 Activation of three different NTR specific fluorogenic probes by HCT-116 cells expressing NfsA_Ec wild type and mutant proteins. 300,000 (FSL76 and FSL178) or 100,000 (FSL61) cells were seeded into each well of a 6 well plate and incubated with $50 \mu \mathrm{M}$ of probe for 2 hours at $37^{\circ} \mathrm{C}$. Images were taken using a FLoid ${ }^{\circ}$ Cell Imaging Station, with identical settings for each cell line. The relief phase overlay is omitted from images of FSL76 and FSL178 activation to allow a clearer view of probe activation.

\subsubsection{Metabolism of EF5 by HCT-116: NTR cell lines}

Despite concerns that the lowered levels of NfsA_Ec mutant expression might complicate interpretation of results, we sought to determine if the NfsA_Ec mutants were metabolising EF5 to a cell entrapped product when expressed in HCT-116. This was done by seeding cells into a 6 well plate, incubating them with EF5, and then fixing the cells and staining them with the Alexa 488 conjugated antibody ELK 5.1, which binds to 
EF5-protein adducts. The samples were then run on a flow cytometer, and single cell events assessed for Alexa 488 fluorescence.

Shown are results of incubating the cells with $10 \mu \mathrm{M}-\mathrm{hr}$ EF5 (Figure 5-11). The cell line expressing 1_56 showed near a non-detectable ability to metabolise EF5, similar to that seen on expression of NfsB_Ec. The other three mutants do at least show an ability to metabolise EF5 to a cell entrapped product but it is apparent that this activity is not higher than that exhibited by cell lines expressing wild type NfsA_Ec, and if anything show slightly lowered activity. Exposing the same cell lines to $1 \mu \mathrm{M}-\mathrm{hr}$ EF5, did not substantially separate out the wild type and mutant activities (results not shown).

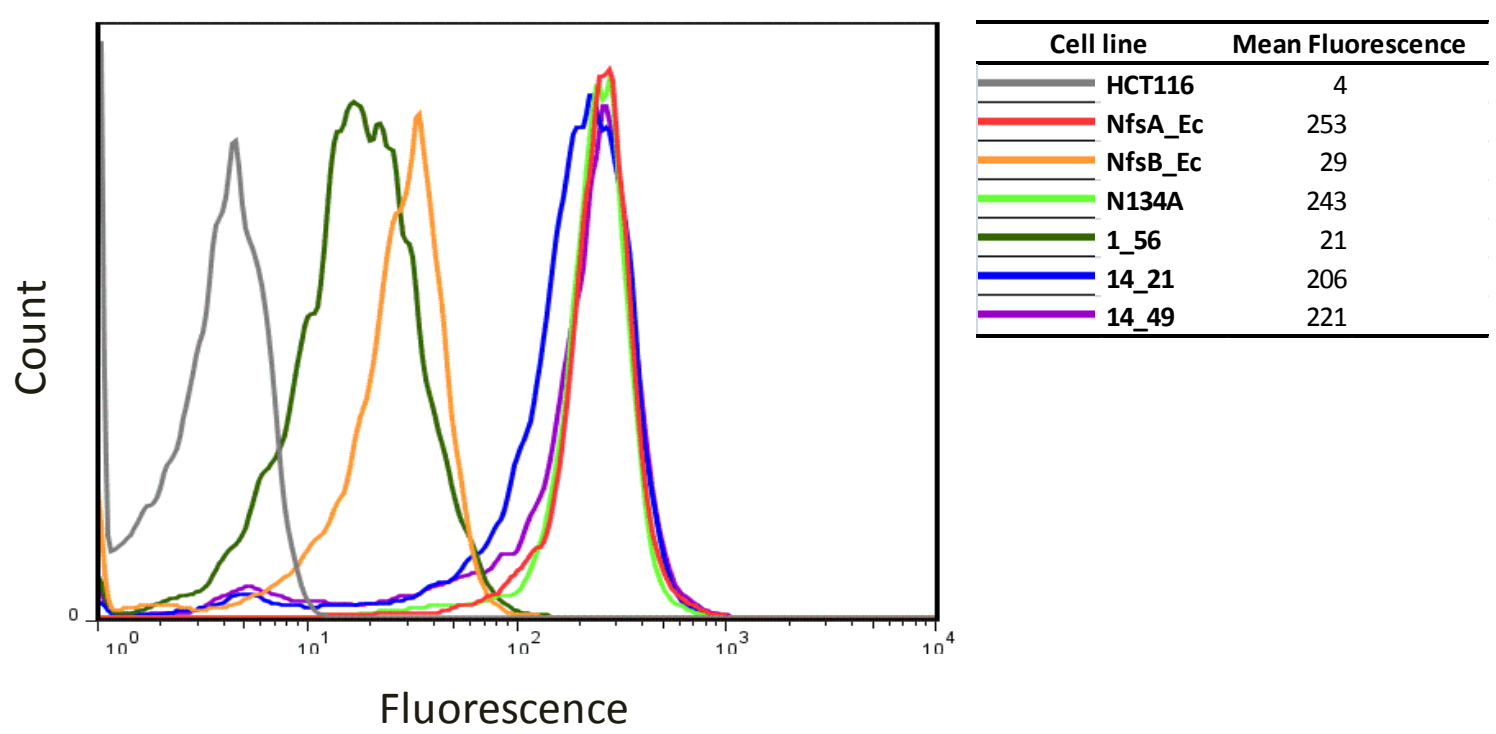

Figure 5-11 Metabolism of EF5 by stable HCT-116:NTR expression cell lines 1 x $10^{6}$ cells were seeded into a 6 well plate and incubated with $10 \mu \mathrm{M}$-hr EF5 at $37^{\circ} \mathrm{C}$. Cells were fixed and stained with Alexa 488 ELK5.1 antibody $(100 \mu \mathrm{g} / \mathrm{ml})$ and analysed on a Becton Dickinson LSRII flow cytometer. Collected data was analysed using FlowJo software and mean fluorescence of each cell line determined. This experiment was repeated twice each time showing the same trends in enzyme activity but with low replication of raw fluorescence values. Displayed are cell population histograms from one representative experiment.

If results are normalised by the levels of NTR over-expression the mutants N134A, 14_21 and 14_49 appear to show greater EF5 metabolism than NfsA_Ec wild type, however such a comparison is not really valid. The protocol used here can quantitatively measure the level of in vivo EF5 metabolism in tumour xenografts seeded with low percentages of HCT-116:NfsA_Ec cells (an example of which can be seen in Figure 3-12). However, considered retrospectively, this protocol has not been optimised for quantitatively 
measuring EF5 metabolism by the 100\% HCT-116: NTR cell cultures used here, (previously with such in vitro cell cultures this protocol has been typically just used to provide a yes/no read out of NTR activity) and it is uncertain if features such as the quantity of antibody employed or the level of overall EF5 exposure would enable linear detection of EF5 adducts over this activity range. Other potential strategies for assessing these mutants will be discussed at the conclusion of this chapter.

\subsection{Results II: Preliminary work investigating a high-throughput screen for identification of mutants with 2-NI improved metabolism}

\subsubsection{A fluorescence activated cell sorting (FACS) based screening strategy}

In addition to the directed evolution work already presented in this chapter preliminary work investigating an alternative strategy for identifying mutants showing improved 2-NI metabolism was carried out. As this was more of a pilot study than a large-scale project, results will be summarised in brief. This strategy uses an adapted version of the SOS assay, in which DNA damage up-regulates expression of GFP rather than $\beta$-galactosidase, to screen for prodrug activating NTRs (Figure 5-12). This was to enable the use of fluorescence activated cell sorting (FACS) to select clones experiencing high levels DNA damage, as with FACS theoretically $10^{6}-10^{8}$ mutant clones can be examined in a realistic timeframe, in contrast to previously presented microplate screening techniques. This system has been successfully used by Dr Janine Copp to find single NfsA_Ec mutants with improved PR-104A metabolism from large random mutant libraries generated through error prone PCR (described in more detail in Chapter 7). As 2-NI imaging agents do not elicit an SOS response NTR activity with these compounds cannot be directly assayed for - instead, the 2-NI compound RB6145, which does generate an SOS response on nitro-reduction, was used as a surrogate compound to screen a large NfsA_Ec mutant library. 


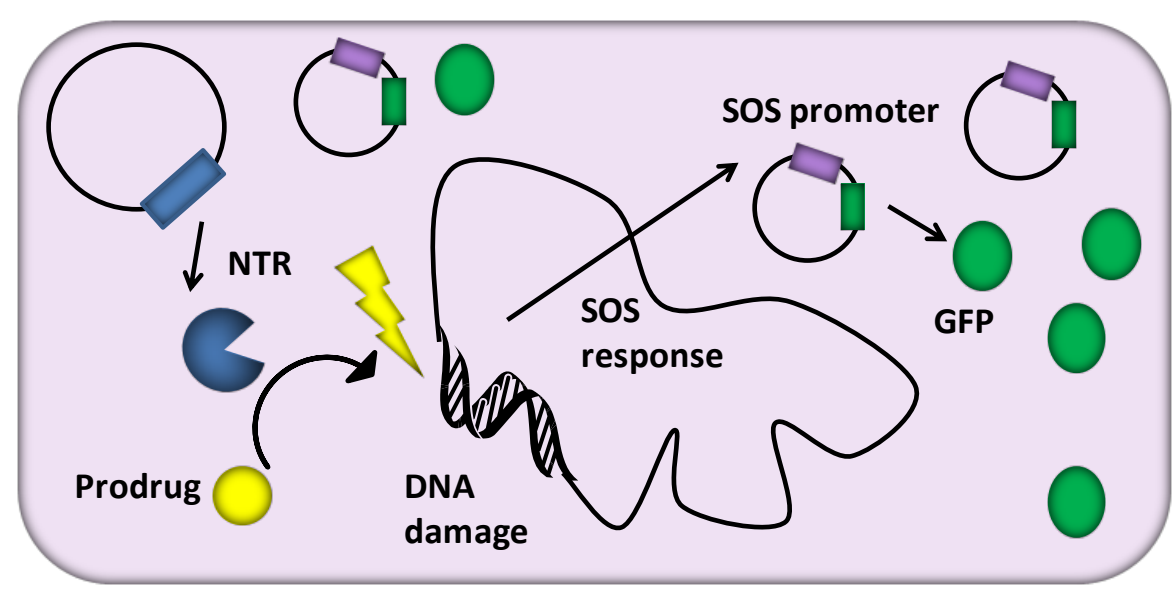

Figure 5-12 Schematic of the GFP-SOS assay designed for the screening of large mutant libraries using FACS. Assays carried out using specialised E. coli strain 7KO (W3110 based strain with seven E. coli oxidoreductase genes knocked out; (see Table 2-2) carrying the plasmid pGFP (Table 2-4) which allows for expression of GFP-mut3 under control of the sfiA/sulA SOS responsive promoter. E. coli strains are challenged with prodrugs and cells expressing NTRs that most efficiently convert this prodrug to a DNA damaging form will trigger the SOS DNA damage repair response to the greatest extent, upregulating GFP-mut3. Cultures expressing different NTRs can then be assessed for prodrug dependent fluorescence using a plate reader or individual fluorescent cells can be enriched using FACS.

\subsubsection{Large NfsA_Ec mutant library fully randomised (NNK codon) at four amino acid sites}

High-throughput screening would enable assessment of libraries which contain fully randomised amino acid identity at more than one site. Such screening could uncover mutations which are only beneficial in combination with other mutant residues (Reetz et al., 2006). These would not be discovered by the previous strategy of combining only a small number of mutations which had all previously yielded improved metabolism at a single mutation level. A mutant gene library which contained NNK codons at sites 41, 45, 83 and 134 was cloned into the pUCX plasmid and validated in a similar manner to that described in section 5.2.5 - sequences of five random clones confirming correct library assembly can be seen in Appendix 3. This simultaneous randomisation gives theoretically 1,048,576 possible variants (calculated using the CASTER mutagenesis tool (Reetz and Carballeira, 2007)).

\subsubsection{FACS selection of GFP expressing cells post RB6145 challenge}

This plasmid library was electroporated into the 7KO pGFP E. coli strain. 7KO is W3110 based strain with seven E. coli oxidoreductase genes knocked (Table 2-2) carrying the plasmid pGFP (Table 2-4) which allows for conditional expression of GFP-mut3 protein under control of the SOS responsive promoter sfiA/sulA. $7 \mathrm{KO}$ was used as the host strain 
instead of SOS-R2 to avoid potential interference from the SOS-R2 genomic sfiA/sulA:lacZ phusion. Following plasmid electroporation the $7 \mathrm{KO}$ pGFP cells were grown as a mixed culture in liquid media containing antibiotics to select for positive (pUCX:ntr) transformants, and a portion of this culture used to establish sub-cultures that were then challenged with $1 \mu \mathrm{M}$ RB6145. Post 4 hours of challenge cells were collected then washed and diluted in phosphate buffered saline (PBS). The most strongly GFP expressing cells were then selected by FACS, with 25,000 "events" falling within a gate that demarked the top $10 \%$ of fluorescent events. The cells giving rise to these events were collected and plated on solid media. From this, only $\sim 7000$ colonies were recovered, a $\sim 25 \%$ recovery rate.

\subsubsection{Screening the 2-NI metabolism of FACS selected clones}

Colonies (455 in total) were picked into five 96 well microplates, with duplicate wild type, and S41Y and 14_21 controls included on each plate. Clones showing greater 2-NI metabolism than NfsA_Ec wild type were identified by RB6145 SOS assays followed by EF5, F-miso and HX4 growth inhibition assays. The respective pUCX:ntr plasmids for the eight clones showing the greatest overall 2-NI dependent growth inhibition were transformed into E. coli SOS-R2. IC 50 values for EF5, F-miso and HX4 were determined in parallel with NfsA_Ec wild type and the mutants S41Y and 14_21; Figure 5-13. While all mutants were better able to sensitise E. coli to the 2-NI imaging agents than NfsA_Ec wild type, none were more active than the previously identified 14_21 multi-site mutant and the majority were actually less active than the single mutant S41Y. The DNA sequence of these eight mutants was determined and mutations are shown in Figure

\section{5-13B.}

While none of the new mutants were more active with 2-NI imaging agents than those previously discovered, the sequences returned do show that this method recovers quite different mutants - only seven out of the 32 amino acid residue changes seen had previously been identified as improving 2-NI metabolism. It is possible that if the RB6145 FACS screening strategy used here is better optimised, variants with greater activity could be discovered in the future. 
A)

\begin{tabular}{|c|c|c|c|}
\hline & EF5 IC $\mathrm{C}_{50}(\mu \mathrm{M})$ & F-miso IC ${ }_{50}(\mu \mathrm{M})$ & $\mathrm{HX} 4 \mathrm{IC}_{50}(\mu \mathrm{M})$ \\
\hline NfsA_Ec & $134 \pm 3$ & $105.5 \pm 9$ & $232 \pm 18$ \\
\hline S41Y & $66 \pm 1$ & $40.5 \pm 4$ & $127 \pm 3$ \\
\hline 14_21 & $37 \pm 4$ & $25 \pm 3$ & $63 \pm 3$ \\
\hline NNK15 & $94 \pm 3$ & $59 \pm 4$ & $99 \pm 1$ \\
\hline NNK16 & $50 \pm 5$ & $35 \pm 3$ & $82 \pm 0$ \\
\hline NNK21 & $82 \pm 4$ & $66 \pm 7$ & $146 \pm 4$ \\
\hline NNK27 & $63 \pm 3$ & $41 \pm 1$ & $126 \pm 2$ \\
\hline NNK44 & $106 \pm 12$ & $62 \pm 4$ & $168 \pm 5$ \\
\hline NNK46 & $74 \pm 3$ & $46 \pm 5$ & $116 \pm 4$ \\
\hline NNK72 & $66 \pm 3$ & $46 \pm 4$ & $145 \pm 38$ \\
\hline NNK92 & $76 \pm 1$ & $50 \pm 2$ & $166 \pm 1$ \\
\hline
\end{tabular}

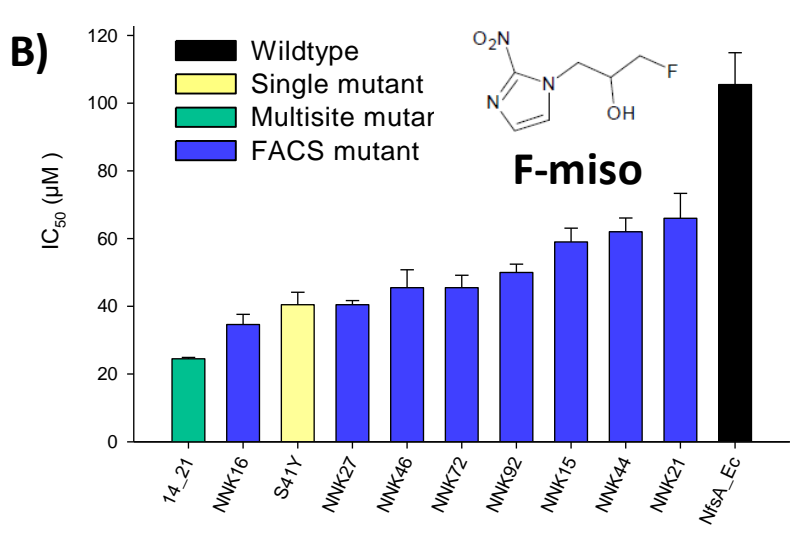

NTR over-expression strain
C)

\begin{tabular}{ccccc}
\hline Mutants & S41X & C45X & F83X & N134X \\
\hline NNK15 & L & H & S & G \\
NNK16 & F & A & L & R \\
NNK21 & L & G & S & I \\
NNK27 & M & S & L & V \\
NNK44 & H & P & G & G \\
NNK46 & L & W & S & A \\
NNK72 & A & - & L & A \\
NNK92 & E & L & F & M \\
\hline
\end{tabular}

Figure 5-13 Mutations and 2-NI metabolism of NfsA_Ec mutants recovered from a FACS sort of an NfsA_Ec 414583134 NNK mutant library. A) EF5, F-miso and HX4 IC 50 values for SOS-R2 E. coli over-expression strains were determined as previously described. Data are the average of two independent experiments \pm SEM. B) To illustrate the mutants' 2-NI metabolism a graph of $\mathrm{F}$-miso $\mathrm{IC}_{50}$ values with strains ordered by increasing $\mathrm{IC}_{50}$ value. $\mathrm{C}$ ) Amino acid residue changes found in clones. Any residue changes which had been previously found to increase 2-NI metabolism when present as a single mutation are marked in red.

\subsection{Discussion}

The work presented in this chapter was carried out to generate NfsA_Ec variants with improved ability to metabolise of 2-NI PET suitable imaging agents. This work has generated a number of multi-site NfsA_Ec variants better able to sensitise E. coli to 2-NI imaging agents. The underlying rationale was that if this activity carries over to a clinical setting, we would be able to image the expression of these NfsA_Ec variants using less ${ }^{18} \mathrm{~F}$-2-NI than would be required to image NfsA_Ec wild type, thus decreasing hypoxic background signal. However we do not yet know if NTR dependent increased E. coli sensitisation to 2-NI imaging agents translates to a measurable increase in 2-NI metabolism on application in a clinical context. 


\subsubsection{Generation of NfsA_Ec variants which show improved 2-NI metabolism on expression in $E$. coli}

To generate NfsA_Ec variants with improved 2-NI metabolism, 12 active site residues identified as likely to be involved in EF5 binding were individually subjected to sitesaturation mutagenesis, with ten of the most promising single mutations then combined in a degenerate gene library. From this eight individual multi-mutant sequences were identified which, when expressed in E. coli, conferred a $3.2-4.8$ fold increased sensitisation to EF5, F-miso, and HX4 compared to NfsA_Ec wild type. As discussed below five of the eight mutants contained either a S41C/L103V/N134A or S41Y/L103V/N134A triple mutation motif.

\subsubsection{Fluorescent activated cell sorting of mutant libraries to identify 2-NI active clones}

Also presented was initial work involving a high-throughput FACS based screening strategy used to assess a portion of a large NfsA_Ec variant library. This screening did not produce variants that were more active than previously identified multi-site mutants, however it is possible that with optimisation this FACS screening strategy may provide more active clones.

Using current protocols the fold enrichment for 2-NI active clones may not actually have been very great. In parallel to the mutant library FACS sort a control experiment was also run, in which cultures of cells expressing either NfsB_Ec (which shows negligible RB6145 metabolism) or NfsA_Ec were challenged with RB6145, mixed in a 9:1 NfsB:NfsA ratio and then this mix FACS sorted for GFP positive cells. Post FACS sort the NfsA expressing cells now made up $\sim 50 \%$ of the tested population, only a five-fold enrichment of NfsA compared to NfsB - results not shown. One feature that could be decreasing recovery of active clones is that the extra toxicity exerted on E. coli cells throughout the FACs process (i.e. the tagging of cells with electric charge) may be preferentially killing cells with highly active NTRs as these will already be experiencing stress due to DNA damage and cytotoxicity from reduced RB6145. That at least some elements of this protocol are toxic to the $E$. coli can be seen by the fact that only $\sim 25 \%$ of sorted "events" generated colonies post FACS. If it was shown that E. coli carrying the most active NTRs are unable to recover post FACS sort this problem could potentially be bypassed by following a strategy of "PCR rescue" in which recovered events are immediately used post-sort as the PCR template for specific amplification of the 
recovered NTRs, which could then be re-cloned back into pUCX vectors and retransformed back into E. coli for downstream testing.

Using previously presented microplate technologies the numbers of clones which can be screened is in the low 1000's and this limitation is probably impacting recovery of highly active variants as only a limited number of residues can be simultaneously mutated at one time. If enrichment ratios using the FACS based protocol can be raised, it is probably worth continuing to assess larger mutant libraries using this strategy.

\subsubsection{Models of multi-site mutants improved in 2-NI metabolism}

The mutation motifs S41C/L103V/N134A and S41Y/L103V/N134A recurred in a number of the multi-site mutants that exhibited improved 2-NI metabolism. To investigate changes these mutations may make to the active site of NfsA_Ec the PyMOL mutagenesis wizard was used to generate models of both of these mutants. Possible explanations for the beneficial effect of the S41C mutation were not readily apparent from the model structure (structure not shown). However pictures of NfsA_Ec wild type (Figure 5-14A), the S41Y/L103V/N134A model (Figure 5-14B) and over-laid predicted modes of EF5 binding (first shown in Figure 5-1) are shown and discussed. It can be seen that in this mutant model the orientation of the Tyr41 residue completely blocks one of the predicted modes of binding. However EF5 binding similar to the second predicted mode may instead be more favoured following S41Y mutation, as here the aromatic Tyr side chain and the FMN could act to sandwich the 2-NI head group in between them. The L103V and N134A mutations could plausibly promote this, as the smaller mutant residues would provide more space for the bulky aromatic Tyr side chain.

While this is only a model of the S41Y mutation and of EF5 binding we have some indication that a similar orientation may occur in the actual protein. A very recently completed crystal structure of the NfsA_Ec S41Y single mutant (overall solved to a high resolution of 1.3 angstrom) shows disorder specifically at the S41Y site, suggesting that the Tyr41 side chain can adopt multiple conformations. That which appears to best fit the densities however is one in which S41Y similarly "sandwiches" a unknown molecule (at this stage tentatively identified as fumarate) between itself and FMN, leading us to speculate that this sandwich conformation may be favourable, and may also occur on binding of aromatic substrates such as PR-104A or EF5 (Dr. Christopher Squire, School of Biological Sciences, University of Auckland, personal communication). 


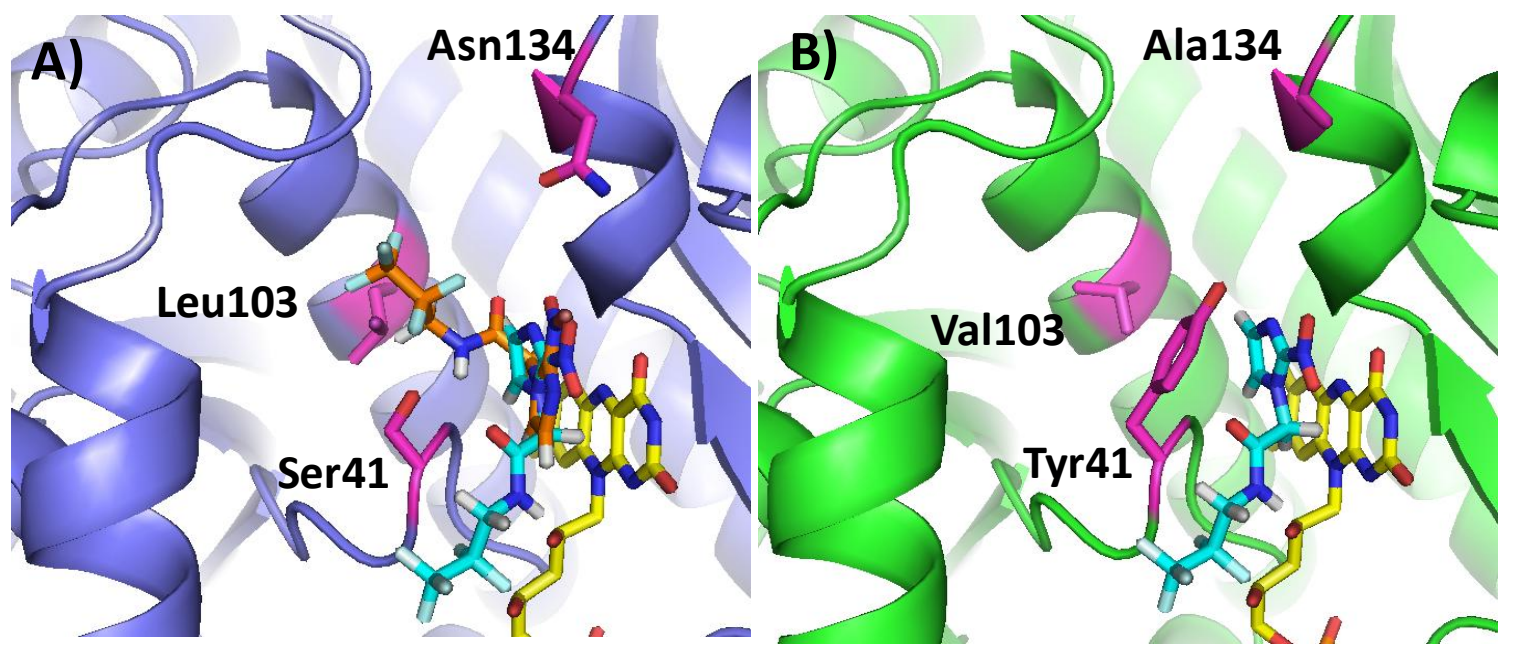

Figure 5-14 Possible effects of S41Y L103V N134A mutations on predicted EF5 binding modes. A) Shown is a representation of the NfsA_Ec crystal structure (PDB code 1F5V) with wild type residues Ser41, Leu103 and Asn134 coloured purple and shown in stick format. Also shown is EF5 in the two predicted modes of binding (orange and cyan carbons) presented in Figure 5-1 and the FMN co-factor (yellow carbons. B) Model of NfsA_Ec S41Y/L103V/N134A (NfsA_Ec variant 14_49) with the 2nd predicted mode of EF5 binding overlaid. Mutant model and images generated in PyMOL using the mutagenesis wizard - each selected residue from the NfsA_Ec crystal structure (1F5V) was mutated and the rotamer which was the most frequently occurring and showed no clashes to neighbouring residues selected. (Schrödinger, LLC).

\subsubsection{Apparent kinetic parameters of NfsA_Ec variants}

When apparent kinetic parameters were determined for the improved mutants, six of the eight single mutants and both of the multi-site mutants tested showed a decrease in apparent $K_{m}$, with the majority of these mutants also showing a larger decrease in apparent $k_{\text {cat }}$ such that the specificity constant was lowered overall. Our laboratory had previously observed, with mutants of FRase I_Vf evolved for improved CB1954 activation, that increases in prodrug metabolism were often driven by decreases in $K_{m}$ rather than increases in catalytic turnover rate (Swe et al., 2012). As will be shown in Chapter Seven, the same trend was seen for NfsA_Ec variants with improved PR-104A metabolism. These observations also fit with those presented in the previous chapter, when assessment of a range of wild type NfsA homologues found that purified protein $k_{\text {cat }}$ in no way correlated with sensitisation of an E. coli host strain to EF5, while a moderate correlation between $E$. coli growth inhibition and apparent $K_{m}$ was observed. It is possible that because our screening strategies incorporate the SOS assay, which is run using low sublethal concentrations of compounds, we are favouring identification of variants which can 
operate at these low concentrations. It should be noted however that there are other possible explanations for this lowered $k_{\text {cat }} / K_{m}$ phenomenon, which shall be raised in the final chapter of this thesis.

If we are indeed favouring selection of mutants capable of maximal metabolism at low 2NI concentrations this may well prove favourable for clinical noninvasive imaging. The concentration of ${ }^{18} \mathrm{~F}-2 \mathrm{NI}$ imaging agent that a bacterial NTR would be exposed to in a clinical context is unknown but it is likely to be very low; PET imaging of hypoxia is conducted using approximately 1000 fold lower concentrations of EF5 than that used in the mouse xenograft experiments shown in Figure 3-11 (Koch et al., 2010).

\subsubsection{Expression of NfsA_Ec variants from HCT-116 cells}

As discussed in the previous chapter, attempts by our collaborators to generate stable HCT-116 cells expressing a range of wild type NTRs were complicated by the fact that NTR expression levels were extremely varied, and in many cases decreased over time. We had not anticipated this occurring on expression of mutant NfsA_Ec proteins, as wild type NfsA_Ec expression was unproblematic. However to date researchers within our collaborative group have attempted to generate 21 different HCT-116:NfsA_Ec variant cell lines and found that the majority of NfsA_Ec variants tested show either absent or lowered expression levels. Four of the 21 NTRs to have been tested are the NfsA_Ec variants presented in this chapter; and of these one (mutant 1_56) failed to express at all, while the other three showed lower expression than NfsA_Ec. The 17 other mutants were tested by Alex Mowday and comprised ten single and seven multi-site NfsA_Ec mutants evolved for increased metabolism of the prodrug PR-104A (generation and screening of these mutants will be overviewed in Chapter Seven). The expression and NTR activity of these 17 cell lines were tested after the time period in culture $(\sim 3$ weeks $)$ required to generate $100 \%$ puromycin resistant cell lines, in which all cells should in theory be expressing an NfsA_Ec variant. However, six out of ten single mutants and four out of seven multi-site mutants showed no/negligible expression; and two single and one multisite mutant showed substantially decreased expression compared to NfsA_Ec wild type (Alex Mowday, personal communication). In summary, out of the 21 NfsA_Ec variants that have been tested thus far, 11 failed to express and a further six showed lower than expected expression levels. 
While different codon usage patterns between species have been known to hamper heterologous protein expression we think it unlikely that the changes at a DNA level have caused the variations in NfsA_Ec mutant expression, as in some cases NfsA_Ec expression is completely destroyed following only one mutation. Additionally two multimutant NfsA_Ec variants can be expressed even though they carry codon changes which, when present in NfsA_Ec single mutants, led to no/substantially lowered NfsA_Ec expression from HCT-116 (Alex Mowday - personal communication). It also does not appear that lowered expression and activity is a consequence of protein instability, at least as measured by thermostability (to be more fully discussed in section 6.2.17 in Chapter 6 . The varied NTR expression levels are also unlikely to be a result of clonal variation in HCT-116 expression levels, as each of our stable cell lines are generated from pooled populations of transfected cells.

The cause(s) of this wide variation in functional expression, brought about in some cases by just one amino acid change, are unknown, but one possibility is that the promiscuous metabolism of certain of these NTRs is cytotoxic to the HCT-116 cells and thus over the weeks that we maintain the cell lines in culture the functional expression of these NTRs is disfavoured. Activity and NTR expression from many 100\% puromycin resistant cell lines has been observed to sharply decrease with time (Alex Mowday, Dr Chris Guise, personal communication). Additionally, a number of NTRs that are completely nonexpressed/inactive after the time period of culturing required to generate the $100 \%$ puromycin resistant cell lines (typically at least 2-3 weeks) will actually show functional activity and expression when tested very shortly after transfection (Chris Guise and Alex Mowday, personal communication and additionally an example of this will be shown in the next chapter). Substrate candidates that the NTRs showing problematic expression could be interacting with to generate cytotoxicity could include flavins, quinones (such as those involved in essential mitochondrial metabolism) or some other as of yet unidentified promiscuous substrate.

\subsubsection{Further testing of the 2-NI metabolism NfsA_Ec variants}

This inability to successfully test mutant NTRs has intensified the need to assess the activity of these enzymes on expression from more clinically applicable vectors. As will be discussed in Chapter Seven we have pursued this by developing a collaboration to test the in vitro and in vivo imaging agent and prodrug activating properties of a number of our bacterial NTRs, when expressed from a clinically relevant Clostridium tumour 
targeting bacterial strain. It is hoped that heterologous NTR expression from a bacterial (as opposed to human) host cell will constitute an environment more consistent with the E. coli host used to screen and select the improved NTR variants. 


\section{Chapter 6: Identification and engineering of nitroreductases that activate a hypoxia resistant PET probe}

\subsection{Introduction}

\subsubsection{Design of a de novo NTR imaging agent with decreased hypoxic activation}

As discussed in previous chapters, non-invasive clinical imaging of bacterial nitroreductase enzymes would greatly aid the development of nitroreductase based suicide gene therapy. This was attempted by repurposing 2-nitroimidazole based PET suitable imaging agents, which we have shown to be activated in an oxygen insensitive manner by a number of bacterial NTRs, primarily those from the NfsA family. While the advantage of using clinically advanced compounds for bacterial NTR imaging cannot be overstated, it is also counter-productive to use an imaging agent where background activation (in this case, by tumour hypoxia) will be in the same location as the ultimate NTR target. We have attempted to simplify this potential issue in two ways, the first being the evolution of NfsA_Ec for more efficient metabolism of 2-NI compounds, to increase the differential activity between human oxygen sensitive NTRs and bacterial NTRs. However another option is to redesign the chemical matter so as to decrease activation by hypoxia.

To achieve this requires a compound that is not easily reduced by endogenous human one-electron nitroreductases. One predictive measure of this is a compound's oneelectron reduction potential. This is an experimentally derived value indicating how easily the compound gains electrons (typically as compared to a normal hydrogen electrode). A higher one-electron reduction potential indicates greater electron affinity and easier reduction of the substrate by human oxygen sensitive NTRs (Olive, 1979; Orna and Mason, 1989; Wardman P., 2001). For a nitro-compound to be well suited for reduction under hypoxia it should show a one-electron reduction potential greater than $-0.450 \mathrm{mV}$ (Denny, 2000). Although other side chains do influence one-electron reduction potential, the location of the nitro-group on a heterocyclic ring is typically the largest determining factor. For nitroimidazole compounds ease of reduction proceeds as $2-\mathrm{NI}>5-\mathrm{NI}>4-\mathrm{NI}$ (Tocher, 1997; Wardman, 1985). As evidenced by their use as hypoxia imaging agents 2- 
NI compounds are within the favourable range for reduction. e.g. misonidazole has a oneelectron reduction potential of $-0.389 \mathrm{mV}$ (Wardman, 1985).

To generate a PET-capable probe our ACSRC collaborator Dr Jeff Smaill designed a 5NI analogue of EF5 intended to give higher specificity as a bacterial NTR imaging agent. This compound was synthesised and termed SN33623 (Figure 6-1). The identical side chain to EF5 was chosen as it was anticipated that the antibodies raised against EF5 would also detect SN33623 binding - as indeed was found to be the case (Adam Patterson, personal communication).
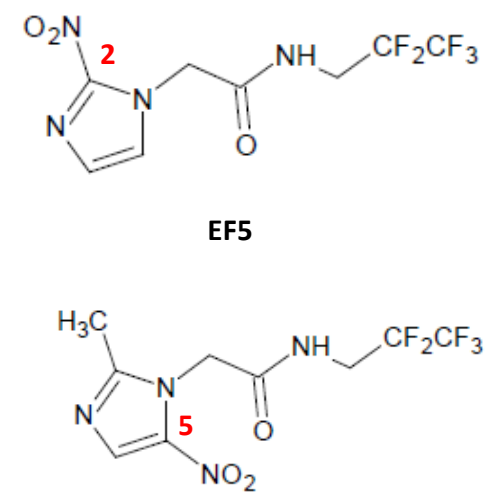

SN33623

Figure 6-1 Chemical structures of EF5 and the de novo potential NTR imaging agent SN33623. Position of the functional nitro group marked in red.

Our collaborators at the ACSRC measured the one electron reduction potential of SN33623 as being - $0.501 \mathrm{mV}$, lower than that of EF5 $(-0.395 \mathrm{mV})$ and outside the range considered to indicate efficient reduction by human one-electron nitroreductase enzymes. When tested in vitro, there were trace levels of SN33623 binding when cells were exposed to the compound under strict anoxia but this was far lower than that seen with EF5, as detected using fluorescently labelled antibodies.

\subsubsection{Reduction of SN33623 by bacterial NTRs}

This chapter presents work assessing the ability of bacterial NTRs to reduce SN33623, when expressed both from E. coli and from the human cancer cell line HCT-116. As will be shown, initial testing of NTR activity with SN33623 indicated that while a subset of NfsB family members could reduce the compound, NfsB_Ec itself could not. As such our 
efforts then turned to creating SN33623 active mutants of NfsB_Ec, using the protein sequences of SN33623 active NfsB homologues to guide targeted mutation.

\subsubsection{Aims}

- Assess the ability of members of the 58-NTR library to reduce SN33623

- Identify key mutations that prevent native NfsB_Ec from reducing SN33623

- Engineer NfsB_Ec mutants which can reduce SN33623

- Evaluate the ability of selected NTRs to reduce SN33623 when expressed from the human cancer cell line HCT-116

\subsection{Results}

\subsubsection{In vivo hypoxia dependent metabolism of $\mathrm{SN33623}$}

This experiment was performed in conjunction with two research collaborators at the ACSRC: PhD candidate Alex Mowday (who carried out all animal handling), and Dr. Maria Abbattista (who operated the confocal microscope).

Prior to commencing this study it was imperative to determine whether the in vivo tumour hypoxia metabolism and subsequent binding of SN33623 was indeed markedly lower than that of EF5. During a research visit to the ACSRC I was involved in one of the experiments designed to assess this. Mice carrying HCT-116 tumours were injected with either EF5 or SN33623 at $60 \mathrm{mg} / \mathrm{kg}$. As an internal control to demonstrate levels of hypoxia present in each tumour the mice were also treated with pimonidazole. As previously stated, pimonidazole is a 2-NI compound that can be used for immunohistochemical detection of hypoxia (Varia et al., 1998). Two hours post EF5/SN33623 treatment the tumours were excised, fixed, and tumour sections cut and mounted on glass slides. The samples were then co-stained with a Cy5 conjugated antibody to detect EF5/SN33623 binding and a FITC conjugated antibody to detect pimonidazole binding. It has previously been fully determined that there is no crossreaction between these two antibodies (Dr Maria Abbattista, personal communication). EF5 or SN33623 binding is in red, pimonidazole in green and any overlap between the two when images are overlaid seen in yellow (Figure 6-2). As shown by pimonidazole binding, both tumours have clear hypoxic regions, as would be expected. EF5 binding is readily detectable and occurs in the same areas of hypoxia as pimonidazole whereas SN33623 exhibits negligible binding due to hypoxia. It should be noted that this 
experiment as it stands does not determine if SN33623 actually penetrates to the tumour this facet will be elaborated on in the discussion.
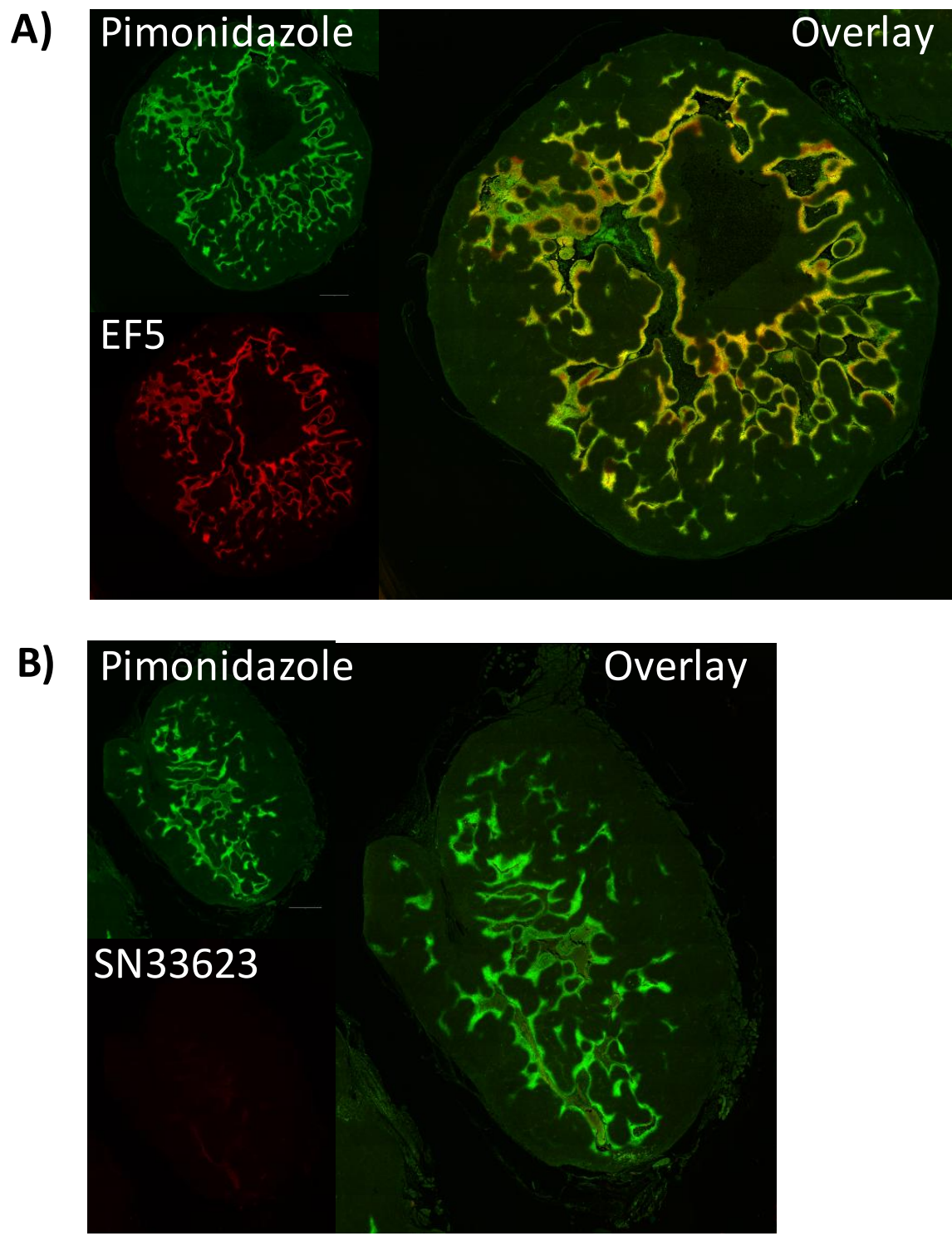

Figure 6-2 Hypoxia dependent binding of A) EF5 and B) SN33623 in a mouse HCT116 tumour xenograft model. Subcutaneous HCT-116 tumours were established in NIH III nude mice and grown to $\sim 10 \mathrm{~mm}$ diameter. Mice were treated with pimonidazole and either EF5 or SN33623 (dosing of all compounds at $60 \mathrm{mg} / \mathrm{kg}$ via intraperitoneal injection) and tumours excised, fixed, paraffin embedded, sectioned and mounted on glass slides for immunohistochemical staining with $(40 \mu \mathrm{g} / \mathrm{mL})$ anti-pimonidazole antibody FITC-Hypoxyprobe-1 (Chemicon ${ }^{\circledR}$ International, clone 4.3.11.3) followed by staining with $(75 \mu \mathrm{g} / \mathrm{mL})$ anti-EF5/SN33623 antibody Cy5-ELK5.1 Images were acquired and overlaid using a Zeiss LSM 710 confocal microscope (x20 magnification - $1 \mathrm{~mm}$ scale marked on pimonidazole images) and Zen2010 software. The separate images taken to detect pimonidazole binding (green) and SN33623/EF5 (red) binding are depicted both individually (left hand panels) and in overlay (right-hand panels; dual registration appearing as yellow). 


\subsubsection{Screening of the expanded NTR candidate library with SN33623}

To determine the most appropriate assay(s) to measure the activity of bacterial NTRs with the potential NTR imaging agent SN33623, its ability to cause both growth inhibition and induction of the SOS response in the core SOS-R2 NTR over-expression library was determined.

\subsubsection{Growth inhibition}

As shown in Chapter 4, strong activation of 2-NI imaging agents is a property restricted to the NfsA enzyme family. In contrast exposing the same NTRs to SN33623 and monitoring growth inhibition revealed a wider activation profile (Figure 6-3). The 5-NI drug was still reduced by the majority of NfsA enzymes, but comparable activity was also seen with five of the $12 \mathrm{NfsB}$ enzymes in the library.

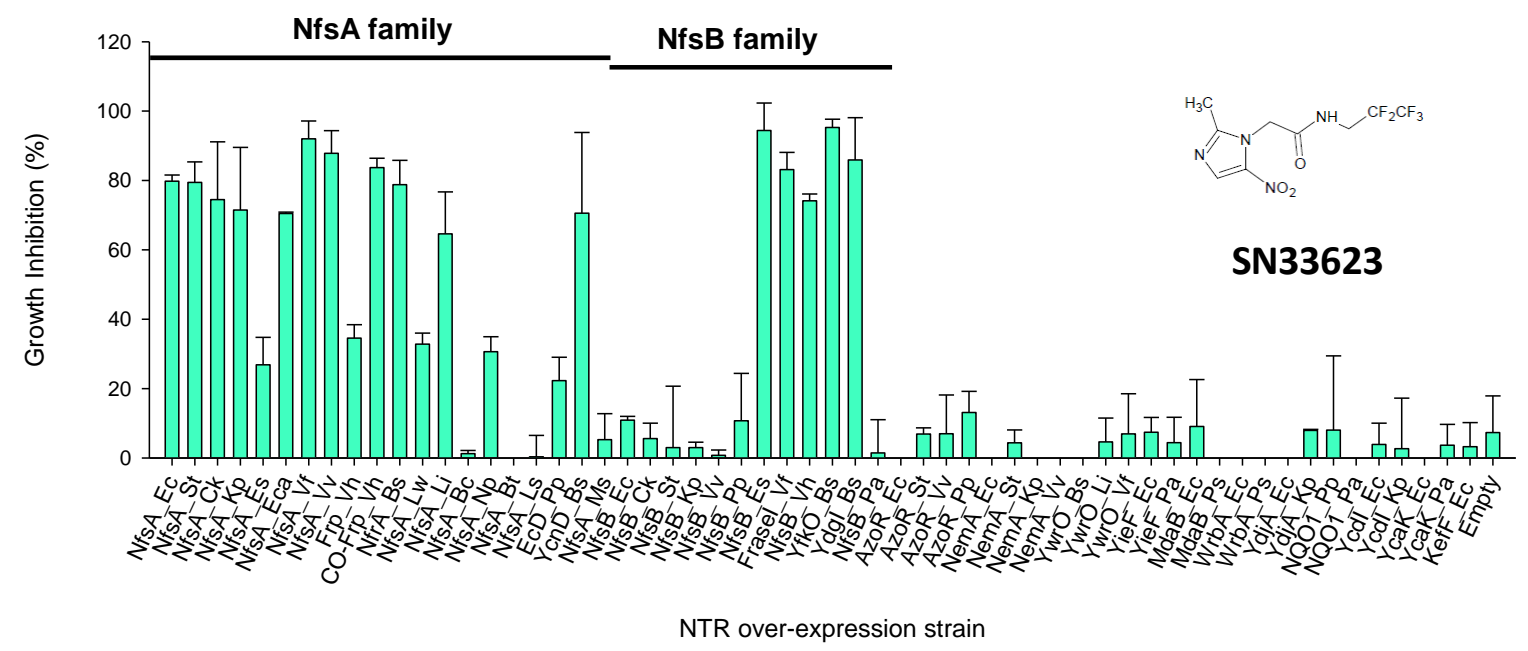

Figure 6-3 SN33623 mediated growth inhibition of SOS-R2 E. coli NTR over-expression strains. Mid-exponential phase over-expression strains were challenged in 384 well plate format with $150 \mu \mathrm{M} \mathrm{SN} 33623$ for 4 hours. Growth was measured as culture turbidity $\left(\mathrm{OD}_{600}\right)$ pre and post drug challenge. Growth inhibition is presented as the percentage decrease in the growth of challenged cultures relative to the unchallenged controls for each strain. Data are the average of two independent experiments \pm SEM.

\subsubsection{SOS response}

In Chapter 3 it was shown that while the reduced forms of the 2-NI imaging agents are cytotoxic at high concentrations, they do not evoke a strong E. coli SOS response. However this was not the case for the 5-NI compound SN33623 which at sub-lethal concentrations could evoke an SOS response in the SOS-R2 NTR library (Figure 6-4). NTR activity with SN33623 as determined by growth inhibition and SOS assay induction 
was closely correlated $\left(\mathrm{R}^{2}=0.91\right.$ for a linear trend line when comparing data presented in Figure 6-3 and Figure 6-4; not shown).

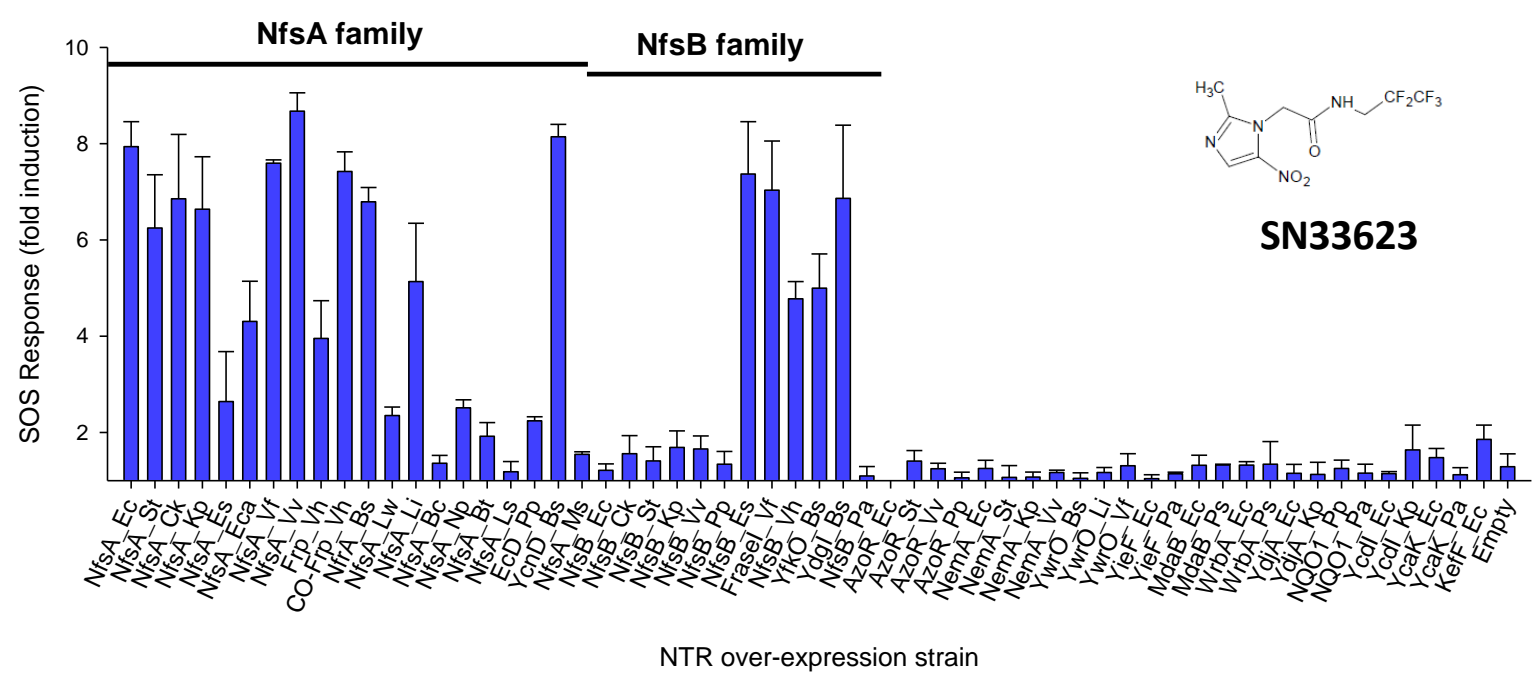

Figure 6-4 SN33623 mediated induction of the SOS response $E$. coli SOS-R2 NTR overexpression strains. Over-expression strains were grown in 96 well microplates and challenged with $8 \mu \mathrm{M}$ SN33623 for 4 hours prior to quantification of SOS response by $\beta$-galactosidase assay. Fold induction is derived from the Miller units recorded on drug exposure divided by those of unchallenged control wells. Data are the average of two independent experiments \pm SEM.

\subsubsection{SN33623 metabolism and evolutionary relationships of NfsB family enzymes}

While the activation of SN33623 by a subset of NfsB enzymes was novel and interesting, NfsB_Ec was clearly unable to metabolise SN33623. This was disappointing as NfsB_Ec is the best-studied and most developed NTR for GDEPT, yet it has never been shown to be imageable. On examining the relationship between all $12 \mathrm{NfsB}$ family members in our library (Figure 6-5) it became apparent that while there was a great deal of diversity between the five NfsBs which were active with SN33623, there was a close relationship (> 50\% shared amino acid identity with NfsB_Ec) between the majority that were inactive (all except for the outlier enzyme NfsB_Pa). This observation suggested that activation of SN33623 may be a general property of the NfsB family of enzymes, with the cluster of NTRs closely related to NfsB_Ec having acquired one or more specific mutations that "block" this activity. If so, we anticipated that it might be possible to engineer in SN33623 metabolism to NfsB_Ec by changing the residue(s) at these site(s) to the equivalent residue(s) from an SN33623 capable NfsB. 


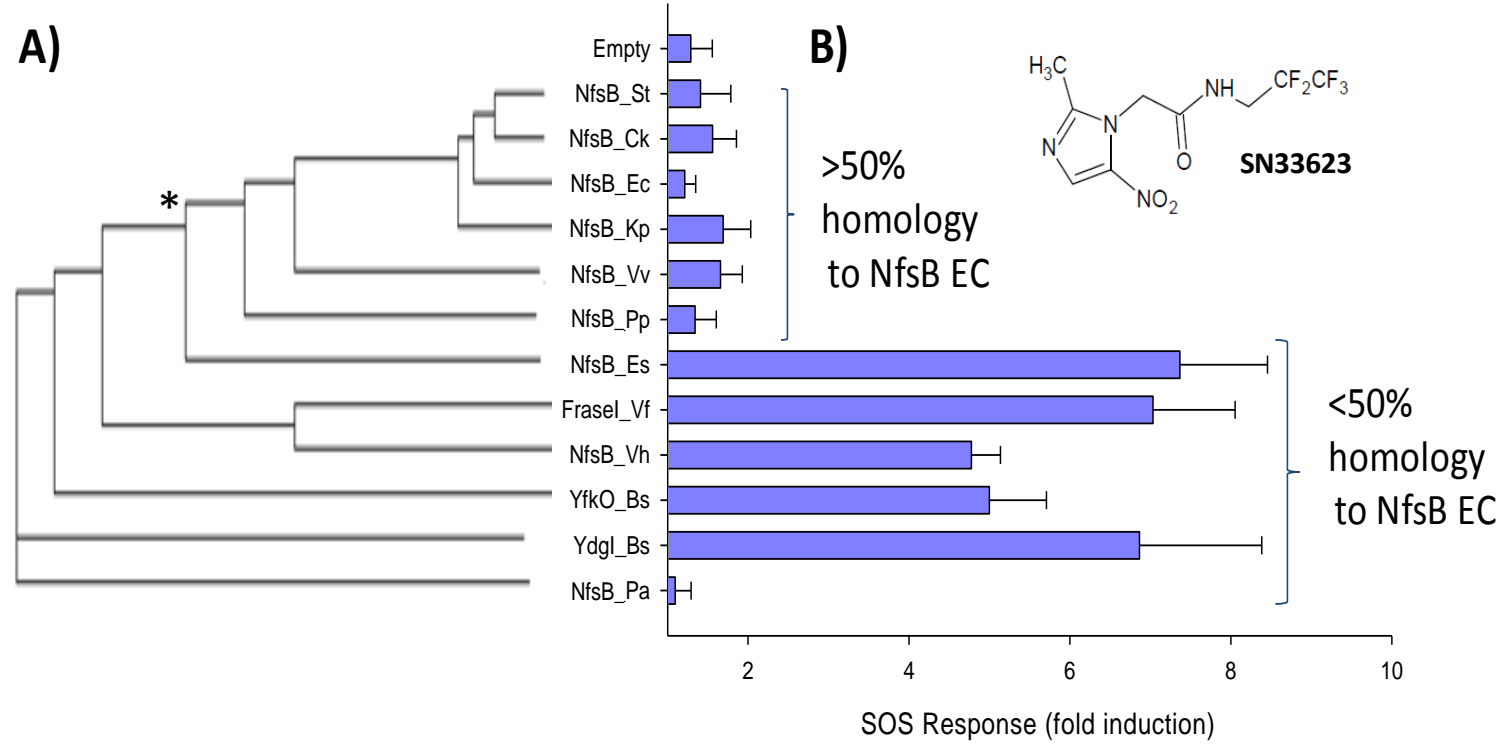

Figure 6-5 Relationships between NfsB family enzymes A) Cladogram representing relationship between the protein sequences of the $12 \mathrm{NfsB}$ enzymes in our NTR over-expression library. Generated using ClustalW2 tool (http://www.ebi.ac.uk/Tools/msa/clustalw2/) on default settings B) Activity of the NfsBs with the imaging agent SN33623 as measured by the SOS chromotest (as seen in Figure 6-4), with NfsBs labelled as to their homology with NfsB_Ec. Homology is defined as $\%$ of shared amino acid identity between the sequences. A* marks point at which NfsB_Es diverges from the other closer NfsB_Ec homologues, past which we hypothesize that mutations blocking SN33623 metabolism occurred.

\subsubsection{Identification of amino acid residues important in SN33623 activation}

To further investigate this idea we compared the protein sequence of the six "NfsB_Eclike" NTRs with that of NfsB_Es, the SN33623 active NfsB that shares the greatest homology (48\% identity) with NfsB_Ec (Figure 6-6 A). As it seemed likely that the amino acid(s) responsible for the change in SN33623 activity would be in the enzyme's active site or binding pocket, we examined a solved crystal structure of NfsB_Ec bound to the prodrug CB1954 and focused only on amino acids within $9.5 \AA$ of the prodrug substrate. This analysis identified 46 amino acids of interest. Of these, 38 amino acid sites could be immediately ruled out as key for SN33623 metabolism, as at these sites NfsB_Es had the same amino acid as at least one SN33623-inactive NfsB. Of the remaining eight sites three were simply a "gap" in the NfsB_Es protein sequence, and modification of the proteins by insertion or deletion in this loop region was not pursued as we decided to first focus on the less disruptive strategy of introducing substitution mutations. This left five sites at which a differing amino acid between NfsB_Es and NfsB_Ec might play a role in determining the ability to metabolise SN33623 (Figure 6-6 B). We decided to focus on the last four of these sites as the change at site 13 (between the structurally similar Ser and Thr) seemed least likely to have a profound effect. 


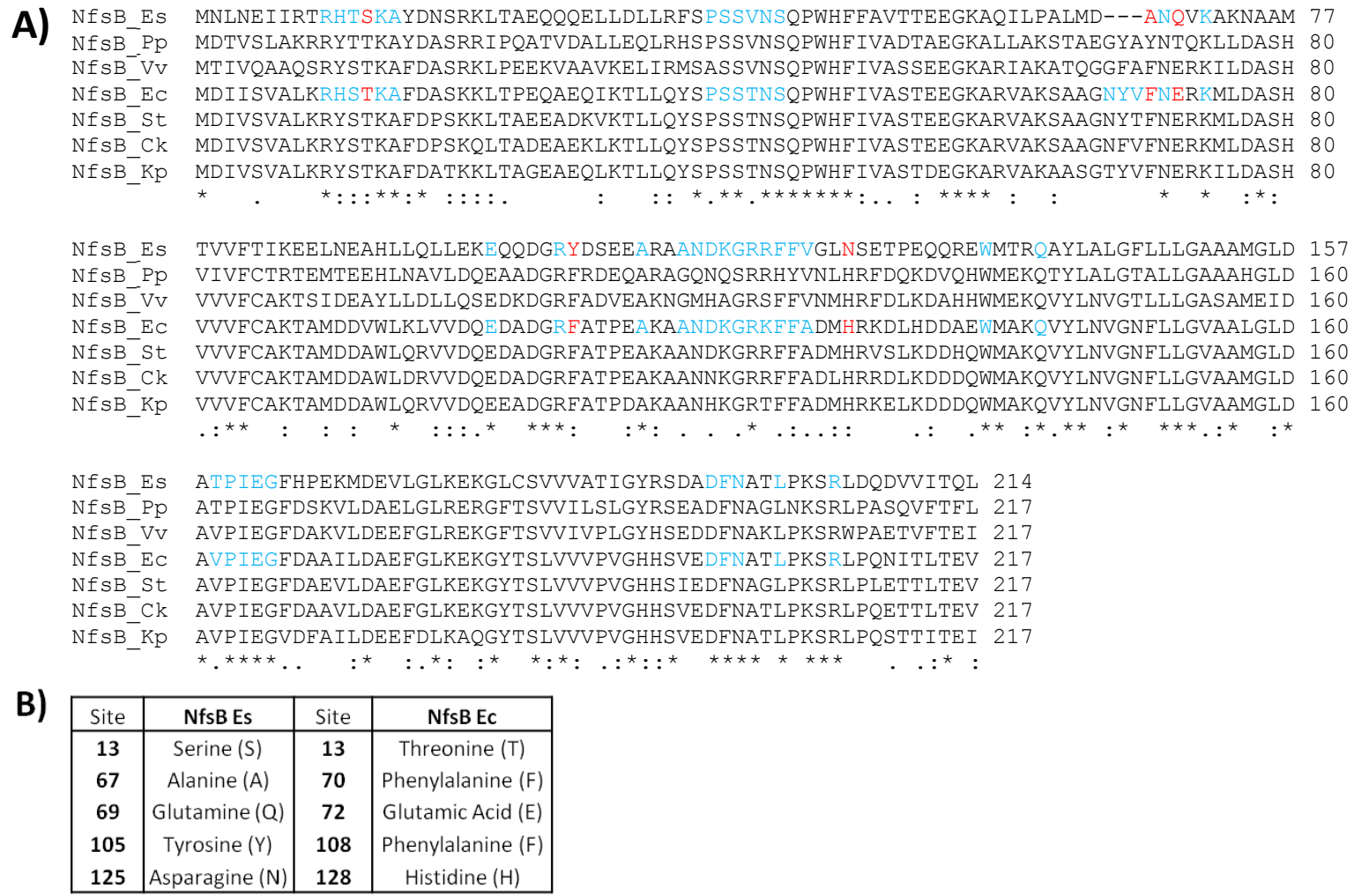

Figure 6-6 Identification of amino acid residues important in SN33623 activation A) Protein alignment of seven NfsB family enzymes. Residues highlighted in blue are those within $9.5 \AA$ of bound CB1954 in NfsB_Ec (determined using the crystal structure 1IDT and protein viewer DeepView (SwissPDB)) and the analogous residues in NfsB_Es. The five residues highlighted in red are those from this group which differ between NfsB_Es and any of the SN33623-inactive NfsBs. Alignment carried out using ClustalW2-Multiple Alignment Tool (http://www.ebi.ac.uk/Tools/msa/clustalw2) on default settings B) Table summarising the five differing residues- differing site numbers are due to the three amino acid "gap" present in NfsB_Es.

\subsubsection{Identification of residues playing a key role in SN33623 metabolism in NfsB_Es}

To determine if any of the four NfsB_Ec amino acids identified in Figure 6-6 B (omitting T13) were blocking metabolism of SN33623 we first substituted those amino acids into the corresponding residue location in NfsB_Es, and assessed if these changes decreased the activity of the resulting NfsB_Es variant with $\mathrm{SN} 33623$. The $\mathrm{SN} 33623 \mathrm{IC}_{50}$ for $E$. coli over-expressing each of the A67F, Q69E, Y105F and N125H single residue variants of NfsB_Es was determined (Figure 6-7). As can be seen, the change which had the greatest effect on SN33623 metabolism was A67F, which eliminated nearly all activity with SN33623. Two other mutations, Q69E and Y105F, also reduced SN33623 metabolism, causing $\sim 2$ fold and $\sim 5$ fold increases in $\mathrm{IC}_{50}$ values, respectively. The fourth mutation, $\mathrm{N} 125 \mathrm{H}$, had no measurable effect on SN33623 metabolism. 


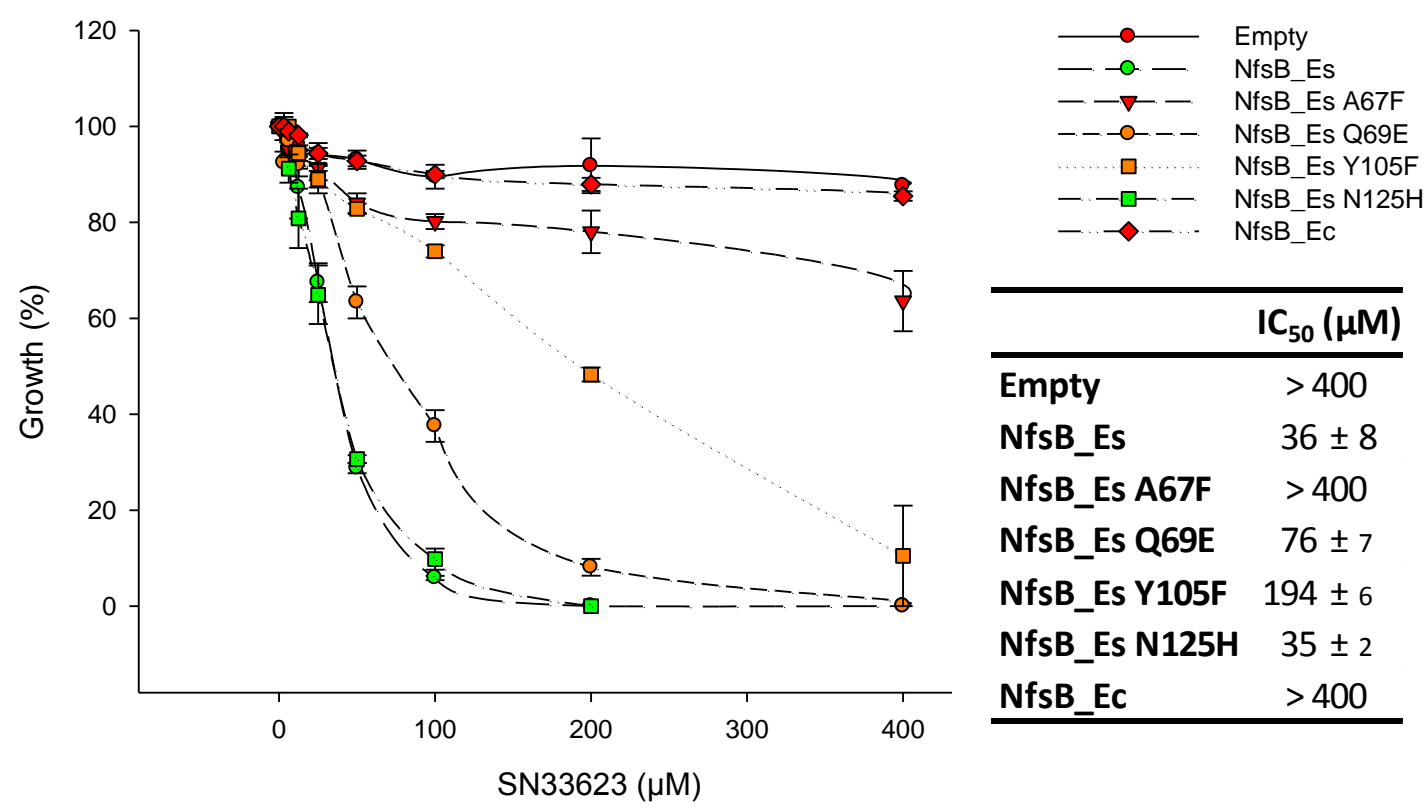

Figure 6-7 SN33623 induced growth inhibition of $E$. coli SOS-R2 NfsB_Es mutant overexpression strains. Growing NTR over-expression strains were exposed to serially diluted SN33623 for four hours. Shown to the left are growth curves, with growth defined as the percentage $\mathrm{OD}_{600}$ increase, compared to unchallenged strain controls, during the drug incubation period. Symbols are coloured green (high activity), orange (medium activity) and red (low to no activity) to denote the relative ability to metabolise SN33623. Inset is a table showing $\mathrm{IC}_{50}$ values, calculated using SigmaPlot ${ }^{\mathrm{TM}}$ 10.0. Values represent the average of three independent experiments \pm SEM.

\subsubsection{Specificity of the decrease in SN33623 metabolism}

To confirm that the observed reductions in NfsB_Es variant activity with SN33623 were a consequence of the specific residue substitutions, and not due to a global deleterious effect on enzyme stability/activity, the ability of these variants to metabolise other compounds was tested. Seen in Figure 6-8 are results with three diverse compounds, the nitroaromatic prodrug PR-104A, the antibiotic nitrofurazone and the nitro quenched fluorophore FSL41 (also known as SN29884 (Singleton et al., 2007)). These results demonstrated that the variant NfsB_Es enzymes retained overall NTR functionality, particularly apparent in the case of PR-104A for which the mutations A67F, Q69E and Y105F actually improved NfsB_Es metabolism. 


\begin{tabular}{|c|c|c|c|}
\hline & \multicolumn{3}{|c|}{$I_{50}(\mu \mathrm{M})$} \\
\hline & SN33623 & PR-104A & NFZ \\
\hline Empty & $>400$ & $1150 \pm 88$ & $>100$ \\
\hline NfsB_Es & $36 \pm 1$ & $910 \pm 26$ & $34 \pm 3$ \\
\hline NfsB_Es A67F & $>400$ & $519 \pm 11$ & $26 \pm 1$ \\
\hline NfsB_Es Q69E & $76 \pm 7$ & $753 \pm 34$ & $29 \pm 4$ \\
\hline NfsB_Es Y105F & $194 \pm 6$ & $675 \pm 51$ & $28 \pm 2$ \\
\hline NfsB_Es N125H & $35 \pm 2$ & $860 \pm 30$ & $41 \pm 4$ \\
\hline
\end{tabular}
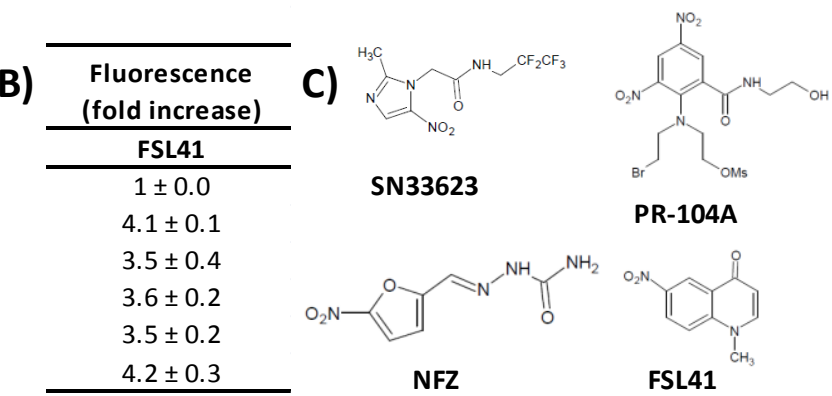

Figure 6-8 Metabolism of SN33623, PR-104A, Nitrofurazone (NFZ) or FSL41 by SOSR-2 $E$. coli strains over-expressing NfsB_Es mutants A) $\mathrm{IC}_{50}$ Values were determined as previously described. Values are the average of at least two independent experiments \pm SEM. SN33623 IC $\mathrm{I}_{50} \mathrm{~S}$ reproduced from Figure 6 for comparison. B) NTR over-expression cultures were grown in 96 well microplates and exposed to $25 \mu \mathrm{M}$ FSL41 (fluorescent when reduced) for 1 hour before culture fluorescence was determined (ex355/em460) and compared to that of unchallenged strain controls. Values are the average of two independent experiments \pm SEM C) Chemical structures of compounds tested.

\subsubsection{Generation of NfsB_Ec mutants that can metabolise SN33623}

As the F67A, Q69E or Y105F mutations in NfsB_Es led to a decrease in SN33623 metabolism it was hypothesised that the opposite substitutions at the equivalent 70,72 and/or 108 sites of NfsB_Ec would generate variants which could activate SN33623. To assess this, all seven possible combinations of F70A, E72Q and F108Y NfsB_Ec variants (i.e. three single mutants, three double mutants, and one triple mutant) were made and tested for SN33623 metabolism (Figure 6-9). It was discovered that changing the amino acids at these sites did indeed engineer in SN33623 activity to NfsB_Ec. The single mutant with the lowest SN33623 IC $_{50}$ was NfsB_Ec F70A, followed by F108Y. E72Q conferred no improvement in activity as a single mutant, but did lower the $\mathrm{SN} 33623 \mathrm{IC}_{50}$ slightly when paired with either of the other two mutations. However the largest synergistic effect came from combining F70A and F108Y as, with $\left(\mathrm{IC}_{50}=38 \mu \mathrm{M}\right)$ or without $\left(\mathrm{IC}_{50}=45 \mu \mathrm{M}\right)$ an accompanying E72Q mutation, NfsB_Ec F70A/F108Y conferred a sensitivity to SN33623 comparable to that of wild type NfsB_Es $\left(\mathrm{IC}_{50}=37\right.$ $\mu \mathrm{M})$. 


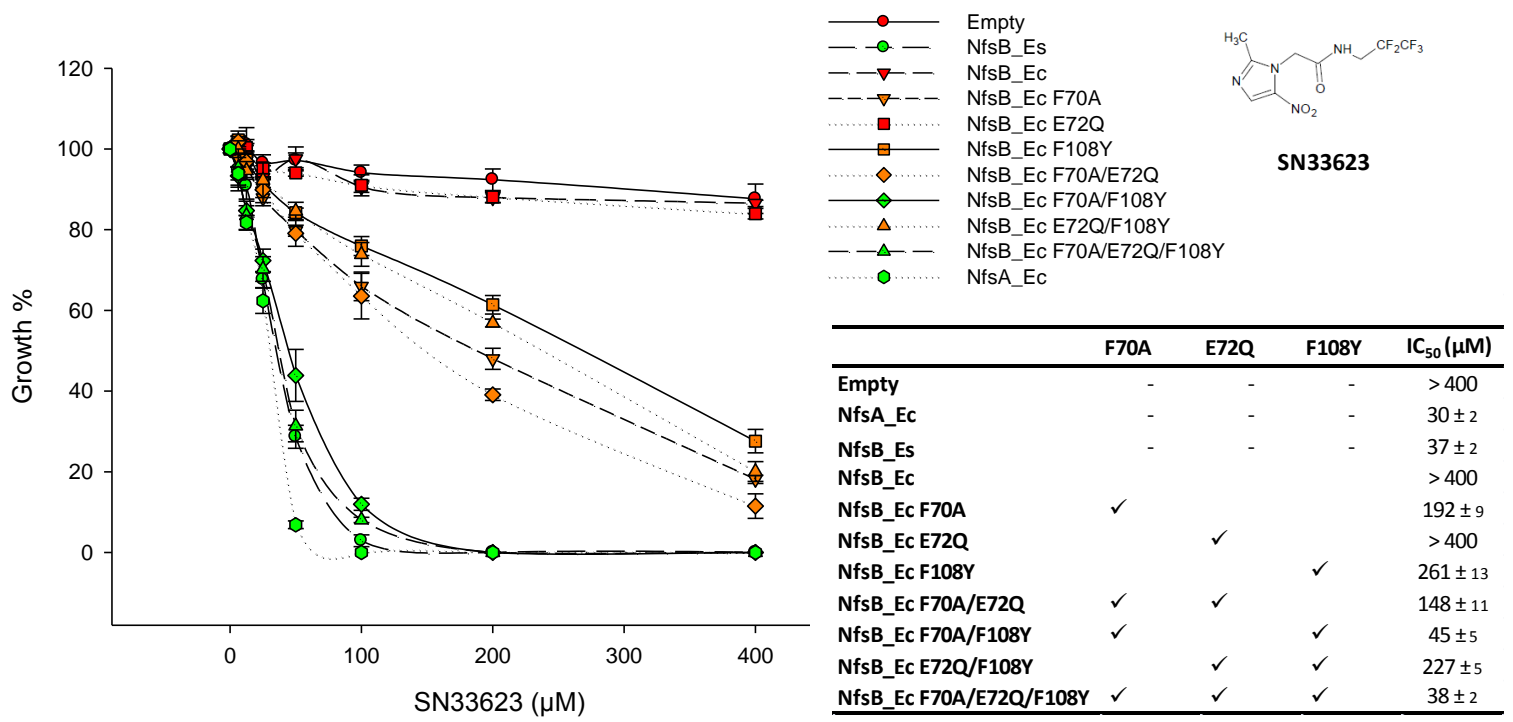

Figure 6-9 SN33623 mediated growth inhibition of $E$. coli SOS-R2 over-expressing engineered NfsB_Ec variants. Mid-exponential phase NTR over-expression strains were exposed to serially diluted SN33623 for four hours. Shown to the left are growth inhibition curves, with growth defined as the percentage $\mathrm{OD}_{600}$ increase relative to unchallenged strain controls, during the drug incubation period. Symbols are coloured green (high activity), orange (medium activity) and red (no activity) to denote the strains relative abilities to metabolise SN33623. Inset is a table indications mutations present in each NTR and strain $\mathrm{IC}_{50}$ values, calculated using SigmaPlot ${ }^{\mathrm{TM}} 10.0$ Values represent the average of three independent experiments \pm SEM.

\subsubsection{Metabolism of 2-NI imaging agents by NfsB_Ec mutants engineered for activity with the 5-NI SN33623}

The HX4 and EF5 dependent growth inhibition of E. coli strains over-expressing NfsB_Ec SN33623 active mutants was tested alongside a strain over-expressing NfsA_Ec, to determine if the introduced mutations also had a positive effect on 2-NI metabolism (Figure 6-10). It was discovered that the four variants containing the F70A mutation had gained a low but nonetheless detectable ability to metabolise EF5 (yielding an $\mathrm{IC}_{50}$ of ca. $1 \mathrm{mM}$, relative to ca. $150 \mu \mathrm{M}$ for NfsA_Ec); whereas the remaining variants were essentially indistinguishable from NfsB_Ec or the empty plasmid control. In contrast, none of the engineered NfsB_Ec variants were distinguishable from wild type NfsB_Ec in terms of conferring sensitivity to HX4 (but all strains expressing these variants were markedly more sensitive to HX4 than the empty plasmid control; yielding $\mathrm{IC}_{50} \mathrm{~S}$ of ca. $800 \mu \mathrm{M}$, relative to ca. $600 \mu \mathrm{M}$ for NfsA_Ec, and $\gg 1 \mathrm{mM}$ for the empty plasmid control). 
A)

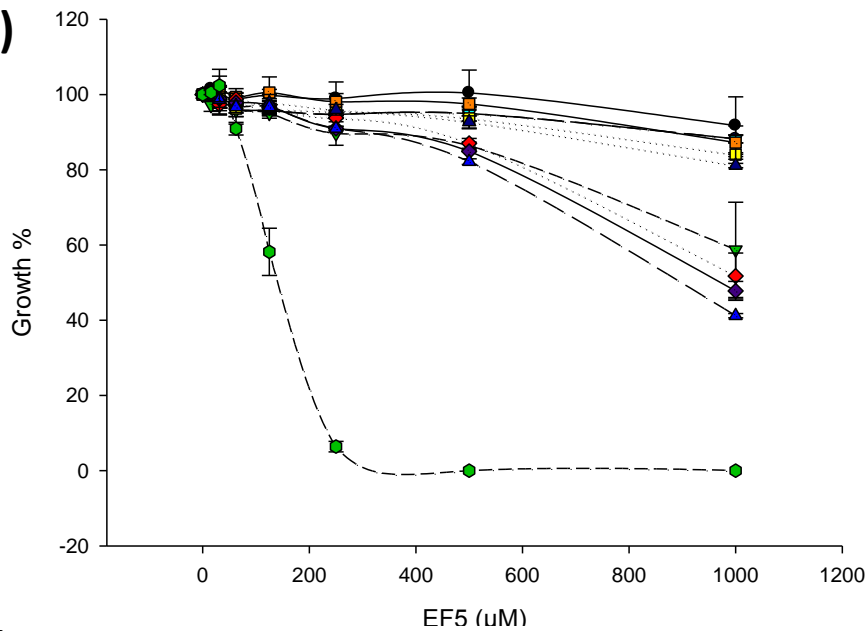

B)

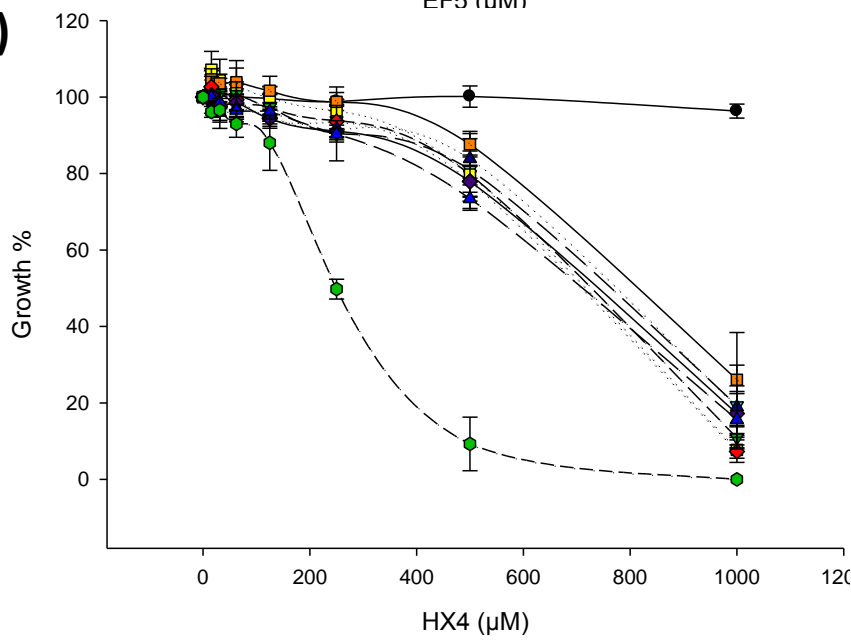

$\longrightarrow$ Empty

$-\nabla-\quad$ NssB_Ec

- - $\nabla$ - - NfsB_Ec F70A

…. NfsB_Ec E72Q

…..... NfsB Ec F70A/E72Q

NIB_EC F70AE72Q

NfsB_Ec F70A/F108Y

NfsB_Ec E72Q/F108Y

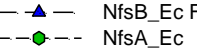

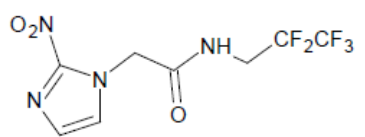

EF5 $\longrightarrow$ Empty

$\longrightarrow$ Empty

- $\nabla$ - - NFB

- NISB_ECF7OA

……… NfsB_Ec E72Q

\begin{tabular}{cl}
$\square$ & NfsB_Ec F108Y \\
\hdashline..... & NfsBEC F70A/E720
\end{tabular}

$\longrightarrow$ NfsB_Ec F70A/F108Y

…....... NfsB_Ec E72Q/F108Y

- A - NfsB_Ec F70A/E72Q/F108Y

- - - N - NsAEC<smiles>O=[N+]([O-])c1nccn1Cc1cn(C(CO)CF)nn1</smiles>

HX4

Figure 6-10 A)EF5 and B)HX4 mediated growth inhibition of $E$. coli SOS-R2 overexpressing NfsB_Ec mutants engineered for improved SN33623 metabolism Mid-exponential phase NTR over-expression strains were exposed to serially diluted EF5 or HX4 for four hours. Shown to the left are growth inhibition curves, with growth defined as the percentage $\mathrm{OD}_{600}$ increase relative to unchallenged strain controls, during the drug incubation period. Values represent the average of two independent experiments \pm SEM.

\subsubsection{Metabolism of SN33623 by NfsB_Ec mutants evolved for superior CB1954 activation.}

Having shown that the amino acid substitutions F70A and F108Y could markedly improve metabolism of SN33623 by NfsB_Ec, we next wanted to see if this knowledge could be used to confer PET imaging potential to variants of NfsB_Ec that had previously been evolved for improved prodrug reduction. For this aspect of the study, two double mutants generated by Dr Peter Searle's research group were chosen, NfsB_Ec T41L/N71S and NfsB_Ec T41L/F70A; these have been reported to be 14-17 fold more potent than wild type NfsB_Ec at sensitising human cancer cells to CB1954 (Jaberipour et al., 2010). We hypothesised that the serendipitous occurrence of the F70A substitution 
in one of these mutants may have already conferred a moderate level of activity with SN33623 to this variant.

The effect on SN33623 and CB1954 metabolism of adding the mutations F70A and F70A/F108Y to Dr Peter Searle's previously evolved enzymes was tested Figure 6-11.

A)

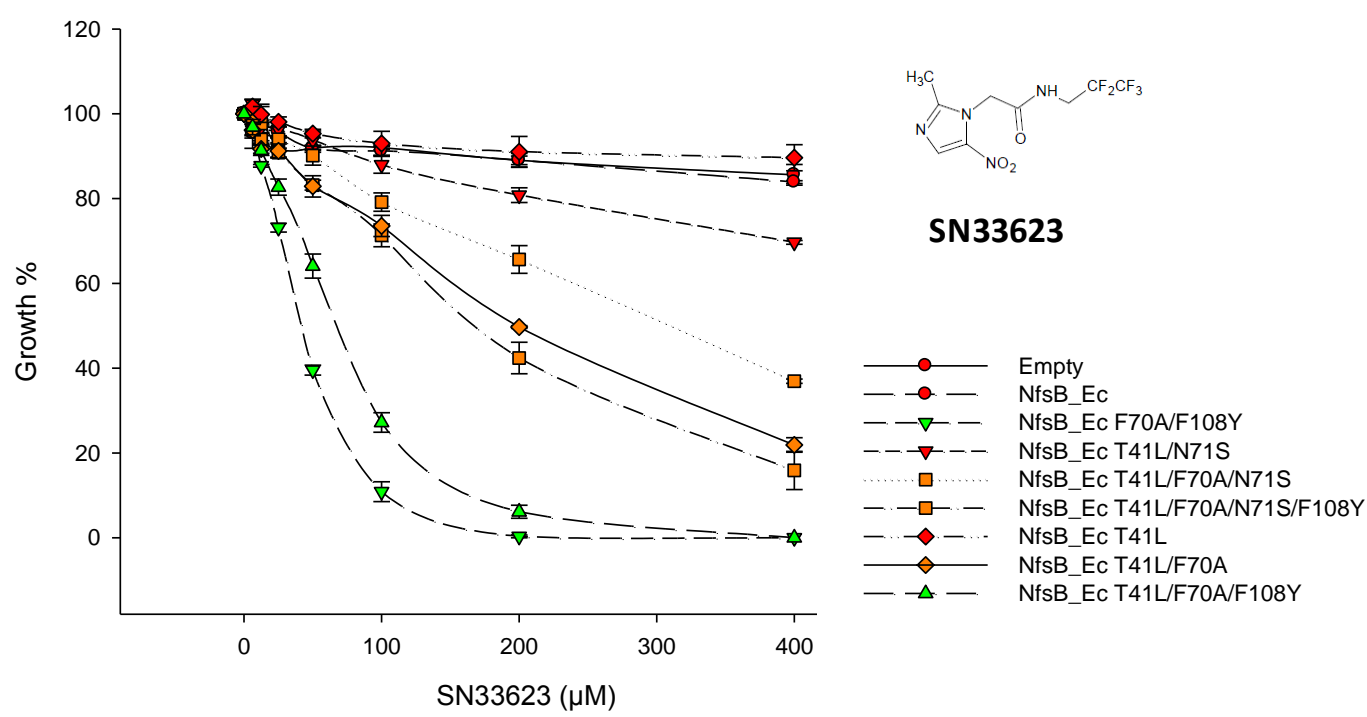

B)

\begin{tabular}{lcc}
\hline & \multicolumn{2}{c}{$\mathrm{IC}_{50}(\boldsymbol{\mu M})$} \\
\hline & SN33623 & CB1954 \\
\hline Empty & $>400$ & $>800$ \\
NfsB_Ec & $>400$ & $376 \pm 6$ \\
NfsB_Ec F70A/F108Y & $41 \pm 1$ & $110 \pm 13$ \\
NfsB_Ec T41L/N71S & $>400$ & $186 \pm 6$ \\
NfsB_Ec T41L/F70A/N71S & $311 \pm 6$ & $246 \pm 10$ \\
NfsB_Ec T41L/F70A/N71S/F108Y & $174 \pm 11$ & $322 \pm 19$ \\
NfsB_Ec T41L & $>400$ & $291 \pm 11$ \\
NfsB_Ec T41L/F70A & $199 \pm 1$ & $229 \pm 25$ \\
\hline NfsB_Ec T41L/F70A/F108Y & $70 \pm 5$ & $201 \pm 15$
\end{tabular}

${ }^{1}$ NfsB_Ec mutant reported by Dr Peter Searle to show improved CB1954 metabolism (Jaberipour et al., 2010)

Figure 6-11 SN33623 and CB1954 mediated growth inhibition of $E$. coli SOS-R2 overexpressing engineered NfsB_Ec mutants A) Mid-exponential phase NTR over-expression strains were exposed to serially diluted SN33623 for four hours. Shown are growth inhibition curves, with growth defined as the percentage $\mathrm{OD}_{600}$ increase, compared to unchallenged strain controls, during the drug incubation period. Symbols are coloured green (high activity), orange (medium activity) and red (no activity) to denote the strains relative abilities to metabolise SN33623 B) Table showing the $\mathrm{IC}_{50}$ s for SN33623 and CB1954. $\mathrm{IC}_{50} \mathrm{~s}$ were derived from the SN33623 growth curves in A) and equivalent experiments using CB1954 and calculated using SigmaPlot ${ }^{\mathrm{TM}}$ 10.0. All values represent the average of three independent experiments \pm SEM. 
When both the F70A/F108Y ("SN33623-improving") mutations were combined with the T41L/N71S ("CB1954-improving") mutations, this did indeed yield an NTR with improved SN33623 metabolism. However, the final $\mathrm{IC}_{50}$ of E. coli over-expressing the quadruple mutant was $174 \mu \mathrm{M}$, substantially higher than the $\mathrm{IC}_{50}$ for the F70A/F108Y double mutant (measured as $41 \mu \mathrm{M}$ in this set of experiments). The addition of F70A and F108Y also compromised CB1954 metabolism, reducing the CB1954 $\mathrm{IC}_{50}$ from $186 \mu \mathrm{M}$ to $322 \mu \mathrm{M}$ (albeit still slightly better than the native NfsB_Ec $\mathrm{IC}_{50}$ of $376 \mu \mathrm{M}$ ). To summarise: when both sets of mutations were combined, the resulting NfsB_Ec variant exhibited better SN33623 and CB1954 metabolism than wild type NfsB_Ec; but substantially less SN33623 metabolism than the specialised F70A/F108Y variant, and substantially less CB1954 metabolism than the specialised T41L/N71S variant.

The strategy was more successful when it came to the T41L/F70A double mutant, which due to the F70A residue change, already had SN33623 activity similar to the NfsB_Ec F70A single mutant ( $\mathrm{IC}_{50} \mathrm{~S}$ of $199 \mu \mathrm{M}$ and $192 \mu \mathrm{M}$ respectively). Adding a F108Y mutation to the T41L/F70A double mutant again proved synergistic for SN33623 metabolism, lowering the $\mathrm{IC}_{50}$ to $78 \mu \mathrm{M}$, and this time not impairing the ability to metabolise CB1954. While neither of these modified mutants were quite as active with SN33623 as the double NfsB_Ec F70A/F108Y, it is possible that the metabolism they do show is sufficient for imaging purposes.

\subsubsection{Reduction of SN33623 by His6-tagged purified proteins.}

To validate the activity of the mutants at purified protein level, the gene sequences were cloned into pET28a+, and purified using nickel affinity chromatography following expression from the E. coli strain BL21. Wild type proteins NfsB_Ec, NfsA_Ec and NfsB_Es were also purified and tested. While we had initially intended to derive the apparent kinetic parameters for each of the NTRs with SN33623 this was complicated by the reduction of SN33623 being extremely slow. Particularly at lower SN33623 concentrations there was only a small difference between background oxidase activity (direct electron transfer from NADPH to dissolved $\mathrm{O}_{2}$ in the reaction medium, measured as the amount of enzyme catalysed NADPH depletion observed in the absence of any additional substrate) and SN33623 dependent NADPH consumption. Comparable levels of slow in vitro activity have been observed by other groups when studying the reduction of the 5-NI compound metronidazole by other bacterial NTRs, and it was found that full determination of kinetic parameters was only possible when using a chemical oxygen 
scavenging system to remove the oxygen from the reaction cuvettes to lessen background oxidase activity (Olekhnovich et al., 2009) (efforts to reproduce this system are ongoing in the Ackerley lab). As a substitute for measuring the full kinetic parameters, the relative rates of NADPH consumption at $500 \mu \mathrm{M}$ SN33623 were determined and these showed that, at least by this measure, the mutations F70A and F108Y did indeed confer SN33623 metabolism to NfsB_Ec.

\begin{tabular}{lcc}
\hline & \multicolumn{2}{c}{$\begin{array}{c}\text { Substrate reduced } \\
\text { ( } \boldsymbol{\mu} \text { moles/min/mg of protein) }\end{array}$} \\
\hline NTR & SN33623 & EF5 \\
\hline NfsA_Ec & $2700 \pm 400$ & $40,700 \pm 1900$ \\
NfsB_Es & $2400 \pm 200$ & $\mathrm{NM}^{2}$ \\
NfsB_Ec & $\mathrm{ND}^{1}$ & $\mathrm{NM}^{2}$ \\
NfsB_Ec F70A/F108Y & $930 \pm 200$ & $\mathrm{NM}^{2}$ \\
NfsB_Ec T41L & $\mathrm{ND}^{1}$ & $\mathrm{NM}^{2}$ \\
NfsB_Ec T41L/F70A/F108Y & $440 \pm 150$ & $\mathrm{NM}^{2}$ \\
\hline${ }^{1}$ No activity detected & & \\
${ }^{2}$ Not measured & &
\end{tabular}

Table 6-1 NADPH consumption rate in the presence of SN33623 by purified His6-tagged NTRs. Purified NTRs were incubated for 1-2 minutes with $500 \mu \mathrm{M}$ SN33623/EF5 and $200 \mu \mathrm{M}$ NADPH. NADPH consumption was determined by monitoring decrease in light absorbance at $340 \mathrm{~nm}$ using an extinction co-efficient of $6,220 \mathrm{M}^{-1} \mathrm{~cm}^{-1}$. NADPH consumption in the absence of SN33623 was determined for each enzyme and subtracted from the final rate value. NfsA_Ec reduction in the presence of EF5 is shown in comparison. Reactions were measured in at least quadruplicate and errors represent \pm one standard deviation.

\subsubsection{Docking of SN33623 and CB1954 into the active mutant NfsB_Es F70A}

To further investigate the ability of the NfsB_Ec mutants to metabolise SN33623 the compound was docked in silico into the active site of a model of NfsB_Ec F70A. A number of X-ray crystal structures of NfsB_Ec have been solved, both unbound (Parkinson et al., 2000) and in complex with other substrates and inhibitors (Johansson et al., 2003; Lovering et al., 2001; Race et al., 2005). NTR amino acid residues are not believed to directly participate in the chemical reactions through which substrate oxidation and reduction occurs. NTRs instead catalyse these by stabilising the charge on reduced FMN and by providing binding pockets to hold the NAD $(\mathrm{P}) \mathrm{H}$ and substrate within an appropriate distance for direct hydride transfer with the FMN co-factor (Koder et al., 2002; Race et al., 2005). 
The NfsB_Ec F70A structure was generated using a solved crystal structure of NfsB_Ec as a template (PDB code 1DS7- (Parkinson et al., 2000)) with residue substitution carried out in PyMOL v1.5 (Schrödinger, LLC). It was decided that docking into a F108Y structure would not be attempted as this mutation site is further than F70A from the FMN binding site and docking into a static model is unlikely to able to detect any effect F108Y may have on substrate binding (Jack Flanagan, personal communication).

NfsB_Ec is a homodimer with two active sites, each formed by residues from both monomers. Docking into both active sites of NfsB_Ec was undertaken, though the two sites are very similar- showing a root-mean-square deviation of only $0.5 \AA$ for all main chain atoms within $5 \AA$ of the FMN molecules (Parkinson et al., 2000).

Firstly the validity of our docking parameters (described in section 2.16) was assessed by docking the prodrug substrate CB1954, to see if we could recapitulate the drug binding orientation observed in x-ray crystallography studies (Johansson et al., 2003) - this was successful as shown in Figure 6-12, providing some degree of confidence around the general modelling strategy as applied to NfsB_Ec.

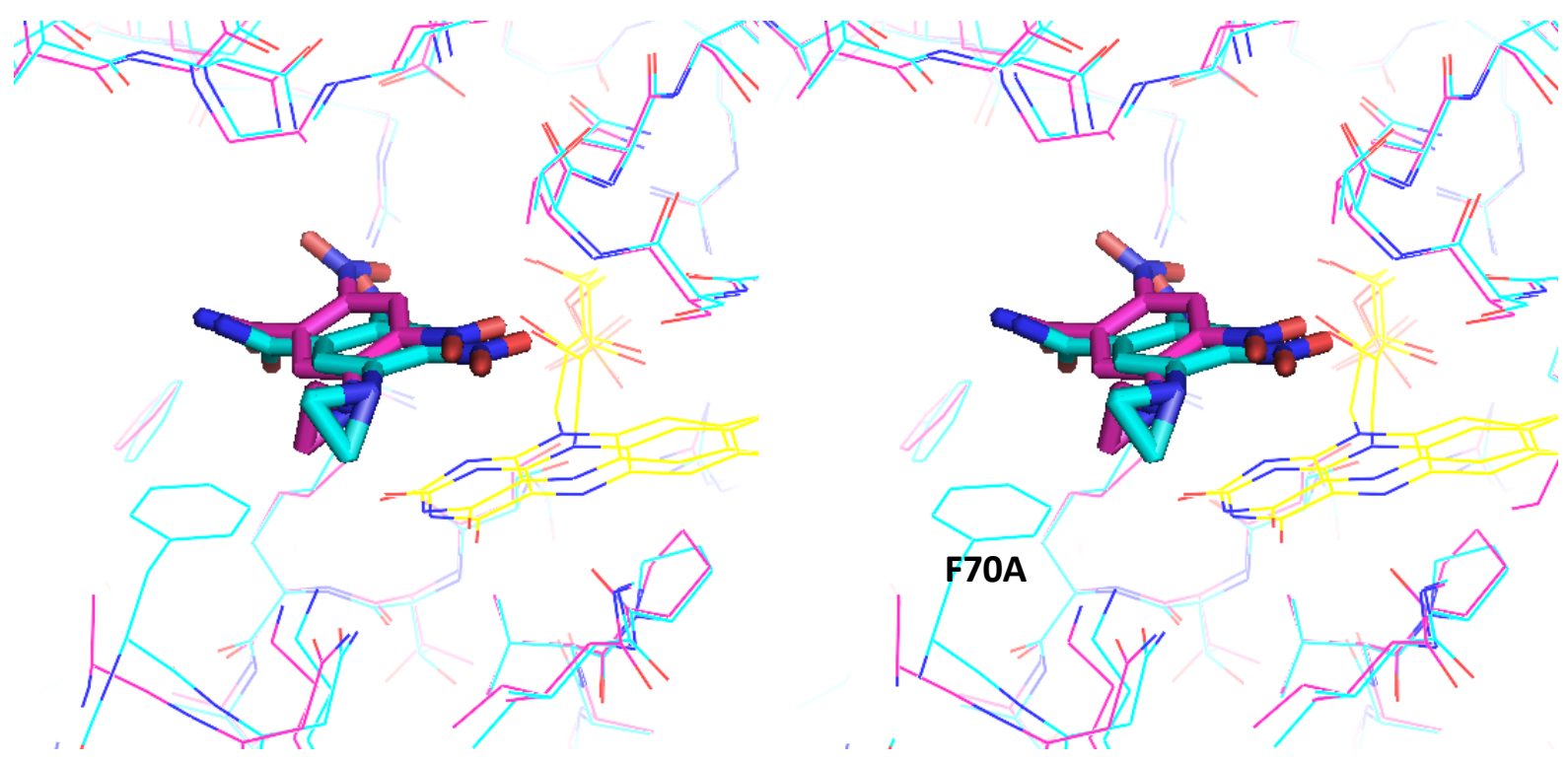

Figure 6-12 Stereo view of CB1954 docked into the NfsB_Ec F70A model (pink carbons), superimposed over X-ray crystal structure of CB1954 bound in NfsB_Ec (PDB code1IDT) (cyan carbons). Site of F70A mutation labelled, CB1954 molecules presented in stick format and FMN co-factors coloured yellow. NfsB_Ec F70A model generated from X-ray crystal structure 1DS7 using PyMOLv1.5, images also generated in PyMOL. Docking performed using GOLDv5.1 and ranked by scoring function ChemPLP. This image shows the first ranked solution for one active site, and similar orientations were seen in the second and fourth ranked solutions for docking into the second active site. 
Next the docking of SN33623 into NfsB_Ec F70A was carried out. Twenty solutions were generated for each active site, and ranked by the scoring function ChemPLP. These were visually inspected for binding orientations which placed the $-\mathrm{NO}_{2}$ functional group of SN33623 close to the FMN cofactor. In the first active site the highest scored solution showed such an orientation, while in the second active site this orientation was seen in both the second and third ranked solutions. In all three solutions the pentafluorine tail of SN33623 was oriented directly toward the space in the structure that arose after mutating the bulky aromatic sidechain of Phe to the smaller methyl sidechain of Ala. Two of the binding orientations observed, which differ in the exact positioning of the pentafluorine tail, can be seen in stereo view in Figure 6-13A. In Figure 6-13C the original NfsB_Ec wild type structure is superimposed over these solutions, demonstrating the manner in which the Phe wild-type residue might block SN33623 binding. 


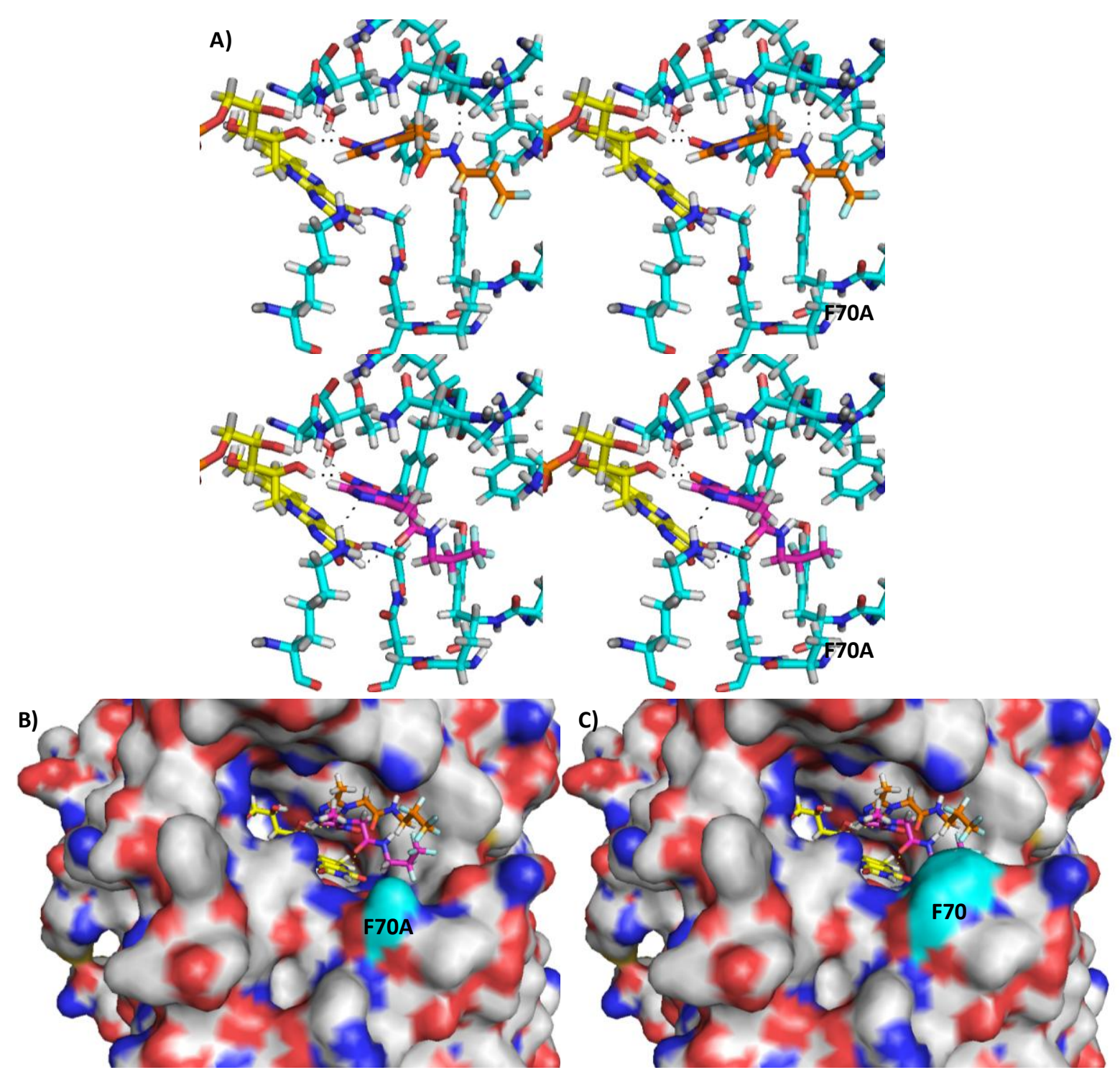

Figure 6-13 Docked structures of SN33623 in NfsB_Ec F70A model A) Stereo images of two different docked orientations of SN33623, shown with carbons in either pink or orange. Putative hydrogen bonds between SN33623 and protein are marked as dashes. The FMN co-factor is coloured yellow. B) Both binding orientations shown in A in a surface representation of the NfsB_Ec F70A model, with mutated F70A residue in cyan. C) Surface representation of X-ray crystal structure of NfsB_Ec (PDB code 1DS7) superimposed on the binding modes shown in A, with Phe70 in cyan. FMN presented in stick form. NfsB_Ec F70A model generated from X-ray crystal structure 1DS7 using PyMOLv1.5. Docking performed using GOLDv5.1. Images generated in PyMOL.

\subsubsection{Testing of bacterial NTR activity when expressed by the human cancer cell line HCT-116}

We next sought to validate that the F70A/ F108Y mutations introduced into NfsB_Ec were truly conferring an ability to metabolise SN33623 to a cell entrapped product and moreover, that this activity could occur on expression of the enzyme from a human cell. As such SN33623 metabolism was tested for these variants (alongside other NTRs) when 
expressed from the human cancer cell line HCT-116. The NTRs chosen for transfection into HCT-116 are shown in Table 6-2, and consisted of three different groups of enzymes: 1) wild type NTRs; 2) variants of NfsB_Ec engineered for "NfsB_Es-like" metabolism of SN33623; and 3) variants of NfsB_Ec enzymes previously engineered by Dr Peter Searle for maximal CB1954 metabolism (including the single mutant NfsB_Ec T41L). As a number of these NTRs were new to this study they required generation of novel F279-V5:ntr constructs and stable HCT-116 NTR expression cell lines.

Stable HCT-116 NTR expression cell lines were generated as previously described, by transfer of the desired NTR sequence into the F279-V5 puromycin resistant expression vector using Gateway ${ }^{\mathrm{TM}}$ cloning, transfection of HCT-116 cells, and selection for plasmid uptake by multiple passage cycles in media containing escalating concentrations of puromycin. HCT-116 NfsA_Ec and NfsB_Ec stable expression cell lines had been previously generated at the ACSRC and were made available for this study.

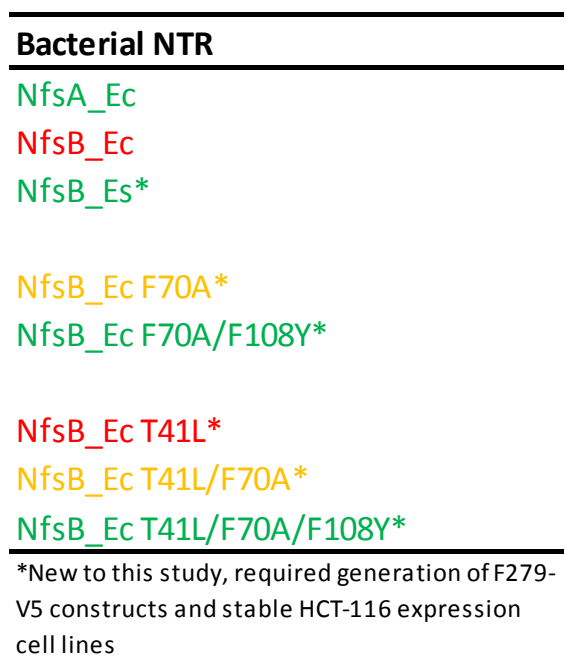

Table 6-2 Bacterial NTRs selected for testing of SN33623 metabolism on expression from the human cancer cell line HCT-116. NTRs coloured according to the $\mathrm{IC}_{50}$ of SN33623 challenged E. coli SOS-R2 NTR over-expression cell lines. Green indicates $\leq 70 \mu \mathrm{M}$, orange 70 $400 \mu \mathrm{M}$ and red $>400 \mu \mathrm{M}$.

\subsubsection{SN33623 metabolism by stable expression HCT-116:ntr cell lines} Once stably-transfected cell lines had been generated for all desired NTRs the ability of those cell lines to metabolise SN33623 was tested. This was done by seeding cells into a 6 well plate, exposing them to SN33623 for 2 hours, fixing the cells and then staining them with the Alexa 488 conjugated antibody ELK 5.1 (as discussed in Section 6.2.1 this antibody recognises both EF5 and SN33623). The samples were then run on a flow 
cytometer, and single cell events assessed for Alexa 488 fluorescence (Figure 6-14). The SN33623 activity of the cell lines expressing wild type NTRs was consistent with the activity they had demonstrated in E. coli, i.e. NfsB_Ec could not metabolise SN33623 while both NfsA_Ec and NfsB_Es could. In contrast, when the NfsB_Ec mutants engineered specifically for metabolism of SN33623 (NfsB_Ec F70A and NfsB_Ec F70A/F108Y) were examined, neither showed any detectable activity with this compound (Figure 6-14A). However, the third group (derived from an enhanced CB1954metabolising variant previously generated by Dr Peter Searle, all containing the additional T14L mutation) were more promising (Figure 6-14B). As expected NfsB_Ec T41L had no activity with SN33623 but both NfsB_Ec T41L/F70A, and to a greater extent NfsB_Ec T41L/F70A/F108Y, did metabolise the compound. 
A)

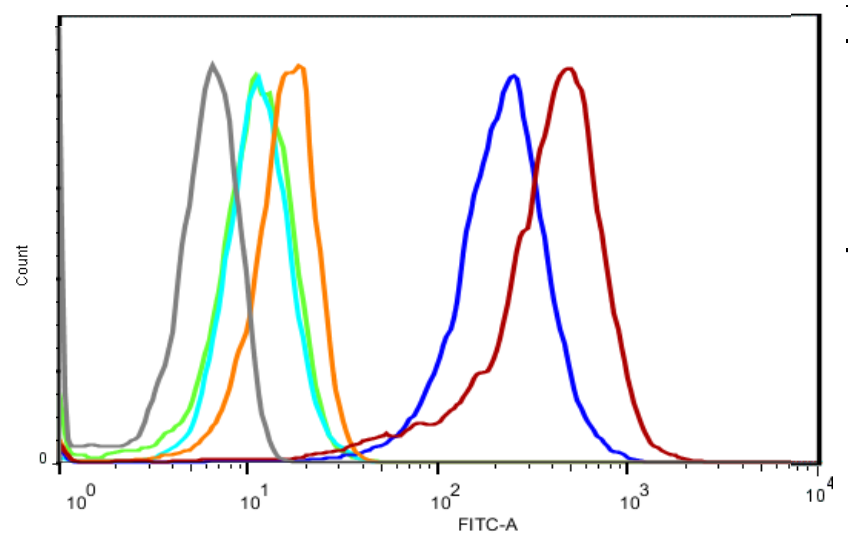

\begin{tabular}{lc}
\hline \multicolumn{1}{c}{ Cell lines } & Mean fluorescence \\
\hline HCT116 & 7 \\
NfsA_Ec & 438 \\
NfsB_Ec & 18 \\
NfsB_Es & 244 \\
NfsB_Ec F70A & 12 \\
NfsB_Ec F70A/F108Y & 12 \\
\hline
\end{tabular}

B)

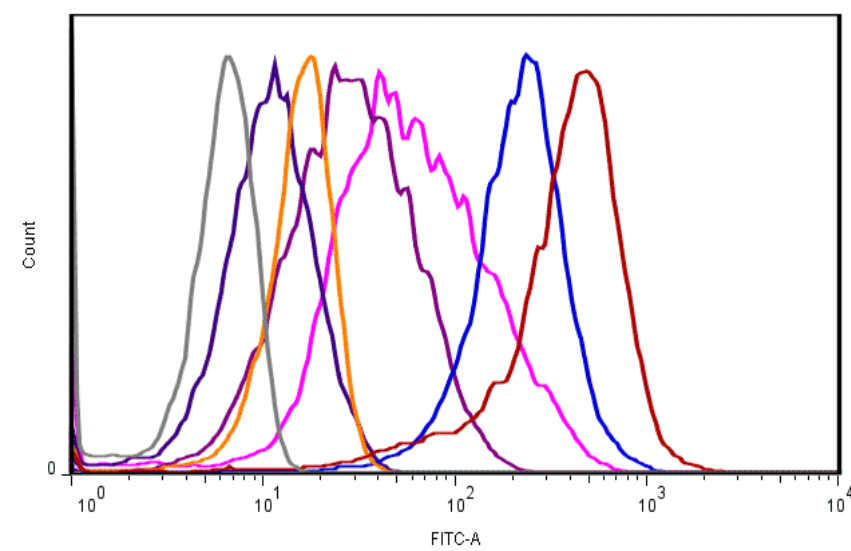

\begin{tabular}{lc}
\hline Cell lines & Mean fluorescence \\
\hline HCT116 & 7 \\
NfsA_Ec & 438 \\
NfsB_Ec & 18 \\
NfsB_Es & 244 \\
NfsB_Ec T41L & 12 \\
NfsB_Ec T41L/F70A & 37 \\
NfsB_Ec T41L/F70A/F108Y & 85 \\
\hline
\end{tabular}

Figure 6-14 Metabolism of SN33623 by stable HCT-116:ntr expression cell lines 1 x $10^{6}$ cells were seeded into a 6 well plate and incubated with $20 \mu \mathrm{M} \mathrm{SN} 33623$ for two hours at $37^{\circ} \mathrm{C}$. Cells were fixed and stained with Alexa 488 ELK5.1 antibody $(100 \mu \mathrm{g} / \mathrm{ml})$ and analysed on a Becton Dickinson LSRII flow cytometer. Collected data was analysed using FlowJo software and mean fluorescence of each cell line determined. For clarity results with cell lines expressing the NfsB variants containing the T41L mutation are shown separately in B) with results of HCT-116 and HCT-116 expressing wild type proteins included for comparison. This experiment was repeated three times over the course of four weeks, each time showing the same trends in enzyme activity but with low replication of raw fluorescence values. Displayed are cell population histograms from one representative experiment. 


\subsubsection{Western blot analysis of NTR expression from stable HCT-116:ntr cell lines}

Immediately prior to SN33623 treatment a subsample of cells was taken and used to determine NfsB_Ec protein expression levels. These samples were lysed and a western blot carried out, probing for NTR expression using a anti-serum raised against NfsB_Ec (Figure 6-15). All of the mutant proteins were found to express at a slightly lower level than NfsB_Ec wild type (1.6-3.8 fold lower than wild type NfsB_Ec as measured by densitometry). However, the NfsB_Ec mutants that had not demonstrated the expected SN33623 metabolism (F70A and F70A/F108Y) were not expressed at a noticeably lower level than those that could metabolise SN33623 (T41L/F70A and T41L/F70A/F108Y).

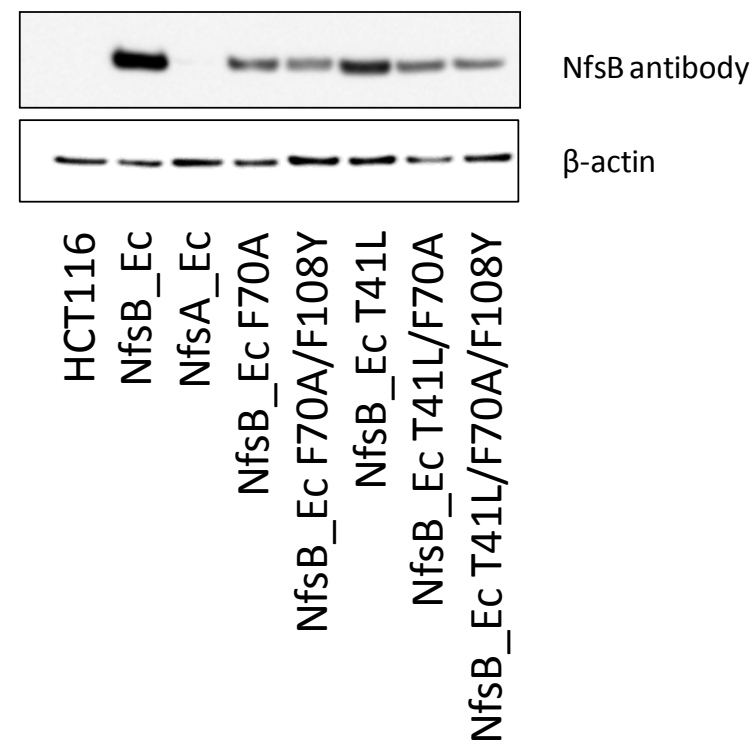

Figure 6-15 Western blot showing expression of NfsB_Ec wild type and mutant proteins from stable HCT-116:ntr cell lines. HCT-116 cell samples were taken, lysed and NfsB_Ec expression determined by immunoblot. $\beta$-actin loading control also shown. This western was conducted using protein samples collected prior to exposure to SN33623, results of which can be seen in Figure 6-14. This trend in expression levels NfsB_Ec $>$ T41L > the four other NfsB_Ec variants were repeated over two western blots of separately taken samples.

\subsubsection{Metabolism of the NTR activated fluorogenic probe FSL61 by HCT-116}

To further interrogate the inability of the NfsB_Ec F70A and F70A/F108Y mutants to metabolise SN33623 all cell lines were tested for an ability to activate the fluorogenic probe FSL61 (an analogue of the previously used FSL41 (Figure 6-8), which is better suited for microscopy as it is more cell entrapped post-activation) (Figure 6-16). Cells were collected, seeded and exposed to FSL61 in 6 well plates, after which media was 
replaced and images taken using the bench top FLoid® Cell Imaging Station. The two mutants that did not contain the T41L mutation showed a markedly lower ability to metabolise FSL61 than the other three mutant proteins, which in turn appeared less avid for FSL61 than wild type NfsB_Ec. This strongly suggested that the NfsB_Ec F70A and NfsB_Ec F70A/F108Y proteins detected in the respective cell lines (Figure 1-14) were non-functional.

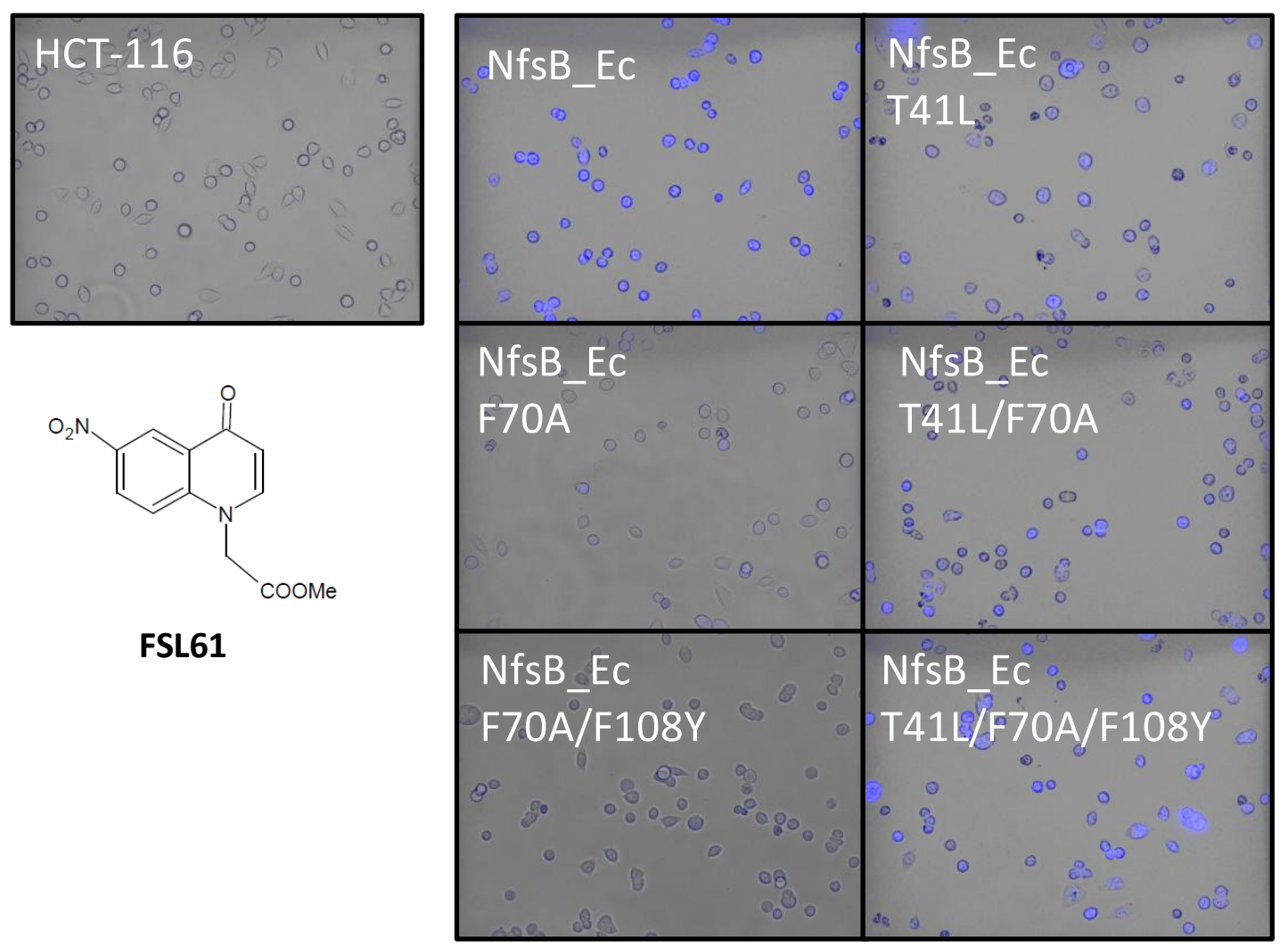

Figure 6-16 Metabolism of the fluorogenic probe FSL61 by stably transfected HCT-116

NTR cell lines. 100,000 cells of the indicated strains were seeded into individual wells of a 6 well plate and incubated with media containing $50 \mu \mathrm{M}$ FSL61 at $37^{\circ} \mathrm{C}$ for two hours. Images were taken in the relief phase and blue fluorescence channel and overlaid using a FLoid® Cell Imaging Station, with identical settings for each cell line.

\subsubsection{Transient expression and exposure to $\mathrm{SN} 33623$}

To confirm whether the observed inactivity of the HCT-116 NfsB_Ec F70A/F108Y cell line was because this NTR is not amenable to long term functional expression in HCT116, the ability of the different NTRs to metabolise SN33623 after transient transfection was tested. HCT-116 cells were transfected with a F279-V5 plasmid expressing either NfsA_Ec wild type, NfsB_Ec wild type, NfsB_Ec F70A/F108Y or NfsB_Ec T41L/F70A/F108Y. Post transfection $(24 \mathrm{~h})$ the cells were collected and to estimate the proportion of cells within each transiently transfected population that were expressing a 
functional NTR an portion of each population $\left(0.5 \times 10^{6}\right.$ cells $)$ were taken, exposed to FSL61 and evaluated for single cell fluorescence by flow cytometry (Figure 6-17A). $1 \mathrm{x}$ $10^{6}$ of remaining cells were exposed to SN33623 for 2 hours, fixed, and stained with anti -EF5/SN33623 antibody. Post antibody staining the SN33623 treated cells were also examined using flow cytometry (Figure 6-17B) and the percentage of each cell population which metabolised the compounds compared (Figure 6-17C). As comparison to the transient expression cells from NfsA_Ec and NfsB_Ec stable expression cell lines were concurrently exposed to SN33623 and FSL61 and analysed in the same manner.

While a moderate proportion (25\%) of cells transiently transfected with the NfsB_Ec wild type plasmid construct were positive for NTR activity (i.e. could metabolise FSL61), only $3 \%$ of these cells were located within the gated region indicating activity with SN33623. In contrast, the NfsA_Ec, NfsB_Ec F70A/F108Y and NfsB_Ec T41L/F70A/F108Y transiently transfected cell lines all showed a markedly higher proportion of SN33623 active cells (39\%-69\%). These results demonstrate that NfsB_Ec F70A/F108Y expressing cells can metabolise SN33623 when tested within 24 hours post the initial plasmid transfection, but not following the long-term culturing required to generate a stable cell line. 


\section{A) FSL61}
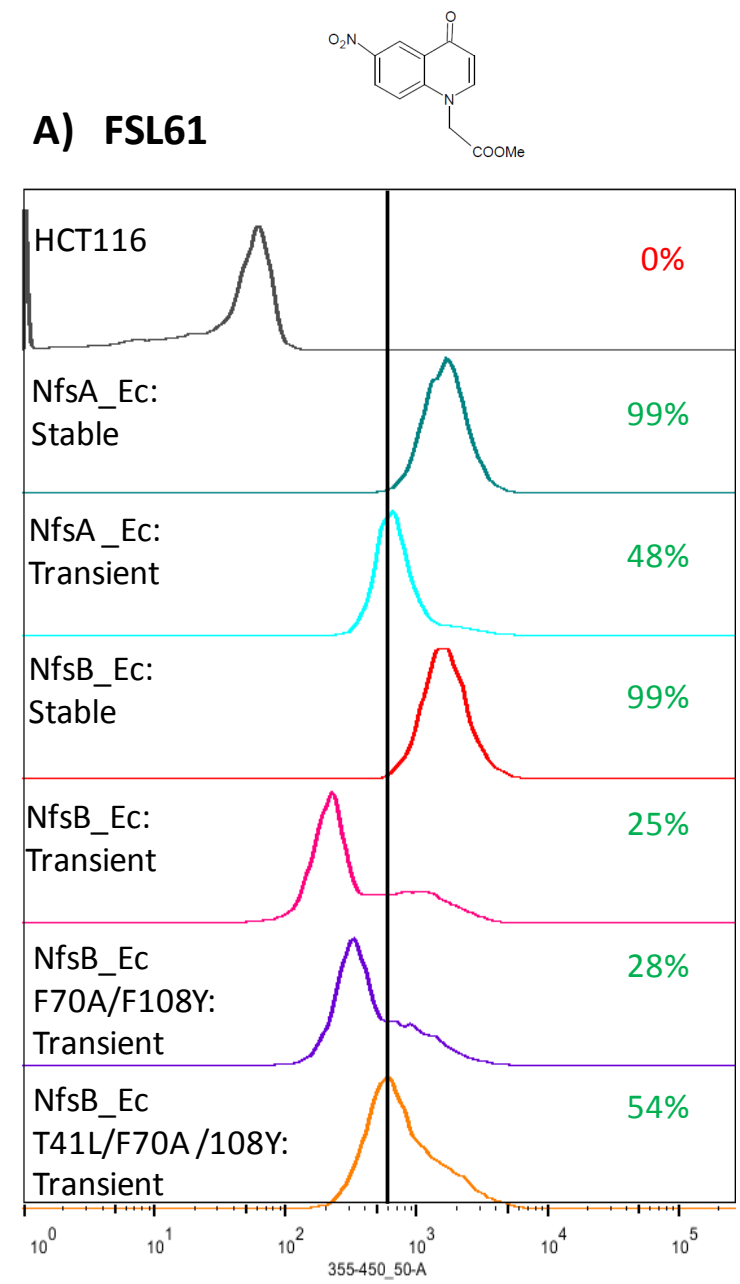

\section{B) SN33623}

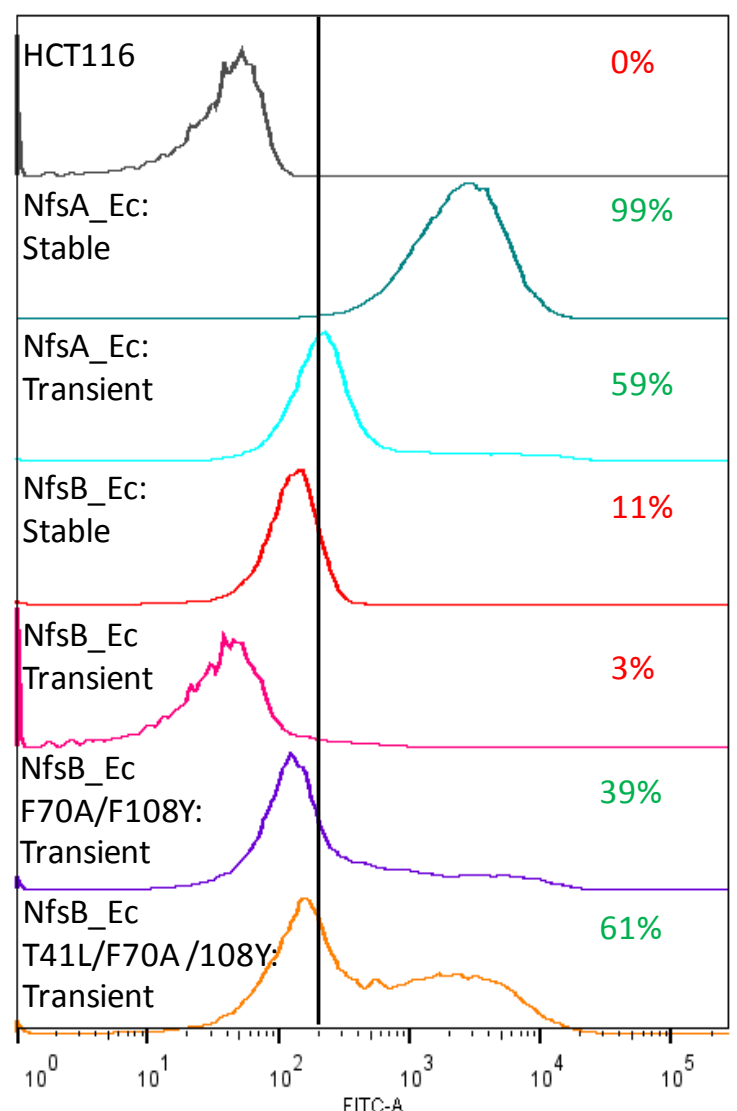

\begin{tabular}{ccc}
\hline & & Cells \\
& $\begin{array}{c}\text { Cells } \\
\text { metabolising } \\
\text { FSL61 (\%) }\end{array}$ & $\begin{array}{c}\text { metabolising } \\
\text { SN33623 (\%) }\end{array}$ \\
\hline HCT116 & 0 & 0 \\
Stable expression: & & \\
NfsA_Ec & 99 & 99 \\
NfsB_Ec & 99 & 11 \\
Transient expression: & & \\
NfsA_Ec & 48 & 59 \\
NfsB_Ec & 25 & 3 \\
NfsB_Ec F70A/F108Y & 28 & 39 \\
NfsB_Ec T41L/F70A/F108Y & 54 & 61 \\
\hline
\end{tabular}

Figure 6-17 Metabolism of A) FSL61 and B) SN33623 by stable and transient HCT-116 NTR expression cell lines. 4 × $10^{6}$ HCT-116 cells were transfected with a F279-V5: $n$ tr construct, and after 24 hours collected and exposed to $50 \mu \mathrm{M}$ FSL61 $\left(0.5 \times 10^{6}\right.$ cells) or $20 \mu \mathrm{M}$ SN33623 (1 x $10^{6}$ cells) for 2 hours at $37^{\circ} \mathrm{C}$. Cells from stable expression lines of HCT-116 NfsA_Ec and NfsB_Ec were also exposed to the compounds. FSL-61 treated cells were immediately analysed on a BD LSRII flow cytometer. SN33623 cells were fixed and stained with antibody Alexa 488 ELK3.51 $(100 \mu \mathrm{g} / \mathrm{ml})$ before flow cytometer analysis. Collected data were compared using FlowJo software. For both data sets a gate was set at the point over which $99 \%$ of the NfsA_Ec stable cell population showed compound metabolism. Cells from other the other samples also within this gate were determined to metabolise FSL61 or SN33623. C) The percentage of each cell line which can metabolise either compound is summarised in table format. 


\subsubsection{Thermostability}

Although western blotting (Figure 1-14) indicated that there are low levels of both NfsB_Ec F70A and NfsB_Ec F70A_F108Y present in stably transfected HCT-116 cell lines, the proteins do not appear to be functional following long term expression. One possible explanation for this phenomenon was that these two mutant proteins are inherently less stable than the NfsB_Ec T41L variants. Of all potential protein stressors, stability in response to increases in temperature is the best studied, and it has been shown in many cases that enzymes with higher thermal stability are also more resistant to other destabilising factors (Burg et al., 1998; Rao et al., 2013). Thermostability can be quantified by a protein's melting temperature $\left(\mathrm{T}_{\mathrm{m}}\right)$, the temperature at which $50 \%$ of the protein is unfolded. The $\mathrm{T}_{\mathrm{m}}$ values of His6-tagged purified proteins were determined using a Protein Thermal Shift ${ }^{\mathrm{TM}}$ assay (Invitrogen, Carlsbad, CA, USA). Here real-time PCR is used to progressively heat the purified proteins which are co-incubated with a Protein Thermal Shift ${ }^{\mathrm{TM}}$ Dye. This is a dye which fluoresces only when it binds to the hydrophobic protein regions exposed as the heated protein unfolds and from these increasing fluorescence levels, measured by the real-time PCR machine, protein $\mathrm{T}_{\mathrm{m}}$ can be determined (Niesen et al., 2007).

\begin{tabular}{lc}
\hline NTR & $\mathbf{T}_{\mathbf{m}}\left({ }^{\circ} \mathbf{C}\right)$ \\
\hline NfsB_Ec & $70.0 \pm 0.0$ \\
NfsB_Ec F70A & $69.6 \pm 0.1$ \\
NfsB_Ec F70A_F108Y & $68.3 \pm 0.1$ \\
NfsB_Ec T41L & $73.6 \pm 0.1$ \\
NfsB_Ec T41L F70A & $74.0 \pm 0.0$ \\
NfsB_Ec T41L F70A F108Y & $73.0 \pm 0.0$ \\
\hline
\end{tabular}

Table 6-3 Melting temperatures $\left(T_{m}\right)$ of His6-tagged purified NfsB_Ec and NfsB_Ec mutant variants Purified proteins were incubated with Protein Thermal Shift ${ }^{\mathrm{TM}}$ Dye and heated in a Rotor-Gene 6000 real-time PCR machine. Increasing fluorescence of the protein preparations was recorded and Rotor-Gene 6000 Series software 1.7 used to generate $T_{m}$ values from the inflection point. Each value is the average of duplicate measures of samples from the same His6tagged protein preparation \pm one standard deviation.

Measured protein melting temperatures were not widely different. It does appear that the T41L mutation has a positive effect on protein thermostability, both when present as a single mutation and in conjunction with F70A and F70A/F108Y and these results do not rule out protein stability playing perhaps a small contributing role in the functional 
activity of the T41L containing mutants as compared to NfsB_Ec F70A and NfsB_Ec F70A/F108Y. However both the F70A and F108Y mutations barely decrease NfsB_Ec stability suggesting that decreased protein stability is not the factor causing in the inactivity of NfsB_Ec F70A and NfsB_Ec F70A_F108Y on stable expression from HCT116.

\subsection{Discussion}

The work presented within this thesis seeks to develop bacterial NTRs as reporter genes for clinical noninvasive imaging of tumour tropic vectors. Whereas previous chapters have focused on repurposing PET probes that were originally developed to image tumour hypoxia, the research described here focuses on a de novo imaging agent, specifically designed to be capable of metabolism by bacterial NTRs, but not by human enzymes under hypoxia. Clinical development of entirely new chemical matter has the substantial disadvantage of requiring expensive preclinical and Phase I clinical trials, whereas repurposing existing PET probes would not incur these costs and time delays. However, effective new chemical matter may prove necessary if existing 2-NI hypoxia imaging agents cannot be successfully repurposed for effective vector imaging. The development pathway for novel radiobiological compounds may also become more simple with time, due to a push for development of PET imaging agents for more varied applications (Coenen et al., 2010) and progress in technologies for miniaturized automated production of varied PET probes (Li and Conti, 2010; Yuin et al., 2012).

\subsubsection{Further investigation of hypoxia resistance and NTR labelling}

The de novo compound presented in this chapter is currently named SN33623, and is the 5-NI analogue of EF5. Critically we showed that SN33623 shows clearly lower in vivo binding in a HCT-116 tumour xenograft. However this experiment does not show that SN33623 actually penetrates tumours as effectively as EF5, and whether SN33623 still retains good ability to give a quantitative measure of NTR expression in vivo. Alex Mowday has recently shown that SN33623 in vivo reduction by NfsA_Ec can be readily detected by immunohistochemistry in a mouse xenograft model of a human lung tumour NCI-H1299, containing approximately 5\% NfsA-expressing cells. Such a result does tell us that the pharmacokinetics of SN33623 are such that NfsA_Ec over-expression can be detected in vivo but for full comparison to the experiment presented in Figure 6-2 this 
needs to be repeated in HCT-116 tumours seeded with varying levels of NTR experiments to determine this are planned at the ACSRC.

When drug binding is measured by fluorescently-conjugated antibodies in vitro under anoxia there is at least 13 fold more fluorescence in EF5 treated cell samples than in those treated with SN33623, however the fluorescence of the SN33623 sample can still be distinguished from that of untreated cells - in vitro it is not a completely anoxia "clean" compound. It should be noted that there are three groups who have done preclinical work developing Gallium-68 or ${ }^{99 \mathrm{~m}}$ Tc labelled 5-NI compounds, with a variety of side chains, as possible hypoxia imaging agents (Das et al., 2003; Giglio et al., 2012; Mallia et al., 2005). These groups do report uptake of the compound in tumours in mice, although when it has been directly compared in vivo to $\left[{ }^{18} \mathrm{~F}\right] \mathrm{F}-\mathrm{MISO}$ the actual tumour uptake and retention of a 5-NI Gallium-68 labelled compound was significantly lower, with the measurable tumour/muscle ratio for the novel imaging agent due to the very rapid clearance of unbound hydrophilic compound from muscle as compared to the more lipophilic F-miso (Fernández et al., 2013). We are in the preliminary stages of investigating the use of 4-NI compounds for NTR imaging, as they have lower oneelectron reduction potentials than even 5-NI compounds. Preliminary results indicate that a 4-NI analogue of EF5 shows less binding under anoxia than SN33623 but that the compound can still be reduced by NfsA_Ec. It should be noted that, while ease of screening should not determine our choice of drug, an advantage of 5-NI compounds is that they elicit an SOS response, something not seen with either 2-NI or 4-NI compounds (preliminary work- results not shown). Directed evolution of NTRs for increased activity with a 5-NI compound would thus be simpler as substantially less scarce imaging agent would be required and active NTRs could be directly assayed for in situations which require high-throughput FACS based screening, such as interrogating large error-prone mutant libraries.

\subsubsection{Bacterial NTRs and SN33623}

A serendipitous outcome of developing SN33623 was an expansion in the variety of NTRs that could potentially be used for noninvasive imaging, as a number of NTRs from the NfsB family showed SN33623 activity comparable to that of NfsA_Ec. Irrespective of whether further development of SN33623 is successful it should at least prove to be a useful pre-clinical tool as it can be activated by a number of NTRs, such as NfsB_Es, which cannot metabolise EF5. 
One NTR that could not metabolise SN33623 was NfsB_Ec. We reasoned that it would be highly advantageous to identify specific mutations that could be introduced into NfsB_Ec, to leverage the wealth of previous research focused on this enzyme, in particular the highly active CB1954 metabolising variants generated by Dr Peter Searle using directed evolution (Jaberipour et al., 2010). While NfsB_Es, the SN33623 active NTR most closely related to NfsB_Ec, shared only $48 \%$ amino acid identity, by aligning NfsB_Ec against all the SN33623 metabolising NfsB family members and focusing only on residues close to the active site the number of likely target residues for mutagenesis was reduced to just four. Ultimately only two amino acid changes (F70A and F108Y) were required to make NfsB_Ec capable of metabolising SN33623. It was shown that these mutations also conferred SN33623 metabolism when engineered into two NfsB_Ec mutants previously reported by Peter Searle to show increased metabolism of the prodrug CB1954. As one of these mutants already serendipitously contained the F70A mutation it was already inherently able to metabolise SN33623, but this property was increased by F108Y addition.

\subsubsection{Expression of NfsB_Ec mutants from HCT-116}

To validate and further study the NfsB_Ec SN33623 active mutants a number of stably transfected HCT-116:ntr cell lines were generated, and NTR function and expression was tested. The results of this work (which was performed in parallel with much of the human cell work presented in Chapter 5) re-emphasise the heterogeneity and unpredictability of NTR expression from HCT-116 cell lines. Particularly dramatic was the strong effect single residue changes can have on the long term ability of NfsB_Ec variants to express functionally in these cells. For example, it was shown that while the F70A mutation (and possibly also the F108Y mutation) had a detrimental effect on long term functional expression, this could rescued to a substantial extent by one additional mutation, T41L.

The apparent expression of F70A and F70A/F108Y in the stable cell lines was comparable to the functional variants of these proteins which contained $\mathrm{T} 41 \mathrm{~L}$, as depicted in the western blot in Figure 1-14. As such F70A and F70A/F108Y are expressed but appear inactive for unknown reasons. One possibility that was tested was if the NfsB_Ec F70A and F70A/F108Y mutant proteins were less stable and had a greater propensity to unfold, with the addition of the third T41L mutation stabilising them. To determine if this was likely the thermostability of each of the purified proteins was measured and while T41L was found to be stabilising this was not a to a particularly marked degree. It is 
perhaps possible that this difference in protein stability, as measured by continuing stability on temperature increase, is exacerbated inside the HCT-116 cellular environment in the face of other stressors, but such a conclusion is fairly speculative. It should be noted that protein thermostability does not correlate with successful stable expression for either the NfsA_Ec variants presented in Chapter 5 (results not shown) or wild type NTRs and the larger set of NfsA_Ec variants for which Alex Mowday attempted to generate stable cell lines (Dr Janine Copp, personal communication).

It is perhaps possible that, if these non-T41L containing NfsB_Ec variants carry out metabolism that is unfavourable to the host human cell as discussed in Chapter 5 , that this is favouring accumulations of mutations in NfsB_Ec F70A and F70A/F108Y which are making the NTRs non-functional. Another speculative possibility for difference in activities between the $\pm \mathrm{T} 41 \mathrm{~L}$ variants is that perhaps they metabolise competing substrates, such as quinones, inside the HCT-116 cells to differing degrees - with perhaps higher quinone metabolism in the non T41L containing mutants inhibiting the metabolism of the exogenously applied FSL61and SN33623 nitro-compounds. This would not however explain why NfsB_Ec F70A/F108Y was functional immediately after transfection but not on long term culturing.

Ultimately measuring the ability of the HCT-116 NTR cell lines to metabolise SN33623 did demonstrate that, in contrast to wild type NfsB_Ec, the NfsB_Ec F70A/F108Y mutants could reduce SN33623. This activity could be detected when measured $\sim 24$ hours after NTR plasmid transfection of the HCT-116 cells or in stable NTR expression cell lines when the mutants also contained the T41L substitution. NTR expression in a therapuetic context from a replicating viral vector would likely be more similar to a transient style transfection situation. It is promising that these mutations could confer metabolism of a potential PET capable probe to previously published CB1954 improved NfsB_Ec variants - in one case without decreasing CB1954 metabolism - as this could in future allow noninvasive clinical imaging of such variants of NfsB_Ec, the enzyme that has been the primary focus of NTR based GDEPT for the past two decades. 


\section{Chapter 7: Directed evolution of NfsA_Ec variants with improved prodrug activation}

\subsection{Introduction}

The primary focus of work presented in this thesis has been to develop the noninvasive imaging capability of bacterial nitroreductase enzymes. However, research in this chapter focuses more on use of directed evolution to generate an optimised PR-104A reducing variant of NfsA_Ec, while retaining its native ability to activate 2-NI PET probes. The key goal was to generate an optimal nitroreductase for PR-104A GDEPT - that has the added capacity to activate PET probes - to facilitate translation of NTR based suicide gene therapy to the clinic. The experimental work presented in this chapter was primarily conducted in collaboration with Dr Janine Copp (Dr Copp focusing primarily on improvement of PR-104A metabolism, while I tested enhanced variants for retention of 2NI PET probe metabolism).

\subsection{Clostridium directed enzyme prodrug therapy}

The intended vector for the optimised enzymes generated in this work was Clostridium sporogenes. Bacteria found within the Clostridium genera are obligate anaerobes which in the presence of oxygen can survive in the form of non-vegetative spores. As such a number of non pathogenic Clostridium species have been investigated as biological anticancer agents- the treatment involving the injection of Clostridium spores which are only able to germinate within the hypoxic and necrotic cores of solid tumours (Morrissey et al., 2010; Umer et al., 2012). Early clinical trials of Clostridium treatment demonstrated that systemic delivery of the spores was safe, that the bacteria germinated within tumours and that in some patients this caused tumour oncolysis - although not to levels sufficient to improve patient outcomes (Carey et al., 1967; Heppner and Möse, 1978). More recent investigations have focused on combination treatments. An attenuated strain of Clostridium novyi, termed C. novyi-NT showed promising preclinical results when combined with both DNA or microtubule targeting chemotherapies (Dang et al., 2001) and with radiotherapy regimes (Bettegowda et al., 2003). A phase I trial to assess the safety of $C$. novyi-NT spores in cancer patients is currently recruiting (http://clinicaltrials.gov/ct2/show/NCT01118819). Another strategy involves "arming" the bacteria with recombinant proteins. This has been made possible by advances over the last decade in techniques for the genetic manipulation of recalcitrant species, such as $C$. 
sporogenes, which show high inherent tumour colonisation properties (Kuehne et al., 2011; Liu et al., 2002; Theys et al., 2006). Proteins chosen for expression have included those expected to either directly trigger an anti-cancer response, for example interleukin-2 (Barbé et al., 2005), or else prodrug converting enzymes. Treatment modalities reported to cause tumour growth delay in preclinical models have used recombinant $C$. sporogenes as an expression vector and include $E$. coli derived cytosine deaminase and 5-FC (Liu et al., 2002), an NfsB homologue from Haemophilus influenza in combination with CB1954 (Theys et al., 2006) and NfsB_Ec coupled with either CB1954 or PR-104 treatment (Liu et al., 2008). In the latter instance, PR-104 was found to generate a substantially greater effect than CB1954 in limiting the growth of NfsB_Ec-labeled mouse xenografts, despite being administered at much lower dose relative to the equivalent human MTD (ca. 1.3fold the human MTD for PR-104, vs. ca. 50-fold for CB1954; Adam Patterson, personal communication). The superiority of PR-104 for nitroreductase GDEPT was therefore clearly established.

\subsubsection{Translation of nitroreductase armed Clostridium to a clinical setting}

In partnership with the ACSRC we have joined an international collaboration seeking to advance NTR GDEPT to advanced preclinical, and then clinical, trial. It is not possible to fully discuss the details of this collaboration, with many aspects of it unable to be disclosed due to a confidentiality agreement signed by all parties. A conservative approach will be taken to avoid breaching the terms of this agreement, including not mentioning the identities or precise roles of the other parties involved. Briefly, we are seeking to stably integrate into a $C$. sporogenes strain an NfsA_Ec variant that has been evolved for maximal PR-104A reduction, while retaining the ability to metabolise 2-NI PET probes, in particular HX4. This is an exciting opportunity to clinically translate not just our work involving nitro-aromatic prodrug activation but also noninvasive imaging, as the collaboration will allow microPET studies of the tumour tracking properties of Clostridium expressing NfsA_Ec variants to be conducted.

In addition to PR-104A and HX4 metabolism the abilities of the NfsA_Ec variants to metabolise metronidazole will also be assessed. Anti-cancer treatment using Clostridium has the advantage that, in the event of adverse events, the bacteria can be cleared from patients with the antibiotic metronidazole, commonly used clinically to treat Clostridium infections (Zar et al., 2007). Metronidazole is a 5-NI compound which has been used for decades in humans, and its toxicity is activated by reduction of its nitro-group (Goodwin 
et al., 1998; Leitsch et al., 2007). This means that if the NfsA_Ec variant introduced into C. sporogenes can also reduce metronidazole the $C$. sporogenes strain will likely become hyper-sensitised to metronidazole treatment - a positive bio-safety control for presentation to clinical trial regulatory committees.

\subsubsection{Evolution of NfsA_Ec for improved PR-104A activation}

To decide which NTRs would be integrated in Clostridium we first focused on identifying mutants with improved PR-104A metabolism and these were then co-screened for retention of activity with HX4. This was done because success of the cancer therapy will be intrinsically enhanced by any improvement in the prodrug activating capabilities of the NTR, while theoretically imaging will be successful as long as a certain (at this point unknown) threshold of NTR activity with HX4 is reached.

Dr. Janine Copp identified single mutations which could be made to NfsA_Ec to improve its metabolism of PR-104A. Beneficial single mutations were found by targeting active site residues using site saturation mutagenesis, in a process similar to that described in Chapter 5. Additional mutations, at sites not previously modelled as being involved in PR-104A binding, were found by screening large random mutation gene libraries generated by error-prone PCR and enriching for positive clones through the FACS based SOS:GFP system previously described (Section 5.3.1). Improved mutants were identified by monitoring for increased SOS response in PR-104A treated E. coli NTR overexpression strains and by measuring PR-104A $\mathrm{IC}_{50} \mathrm{~s}$. Ten identified mutations were then chosen for inclusion into a synthetically constructed mutant gene library (GenScript, $N J$, USA) which, through the use of specifically designed redundancy codons, could code for all possible 512 combinations of these mutations (Table 7-1). 


\begin{tabular}{c}
\hline NfsA_Ec \\
Mutation \\
\hline I5T \\
S41Y \\
E99G \\
L103M \\
K222E \\
R225A \\
R225G \\
R225P \\
F227S \\
L229V \\
\hline
\end{tabular}

Table 7-1 Single mutations to NfsA_Ec determined by Dr Janine Copp to confer improved PR-104A metabolism.

Overall there is little overlap between these ten mutations and those observed in Chapter 5 to improve 2-NI metabolism, with the one exception being S41Y, which was recovered independently in both studies. Thus, it was uncertain whether or not 2-NI metabolism would be retained to a substantial degree in NfsA_Ec variants selected for maximal PR104A metabolism.

\subsection{Aims}

- Assess the 2-NI metabolism of NfsA_Ec single mutants which show improved activity with PR-104A

- Aid Dr Janine Copp in screening an NfsA_Ec multi-mutant gene library to find clones showing improved PR-104A reduction

- Characterise activity of final selected clones with other drugs of importance, principally 2-NI imaging agents.

\subsection{Results}

\subsubsection{Evaluation of the activity of wild type NTRs with HX4}

Successful noninvasive imaging of the therapeutic $C$. sporogenes would require the introduced NTR to reduce ${ }^{18}$ F-HX4. Prior to screening of mutant NTRs the activity of the full wild type NTR library with cold HX4 was examined by measuring growth inhibition post exposure to $200 \mu \mathrm{M}$ or $600 \mu \mathrm{M}$ HX4 (Figure 7-1). Consistent with previously tested 2-NI compounds the majority of NfsA family members (19/20) showed frank activity 
with the compound. A number of NfsB family enzymes (including NfsB_Ec) also showed low levels of activity with the compound.

To confirm that NfsB_Ec has activity with HX4 the abilities of His6-tagged purified NfsA_Ec and NfsB_Ec to reduce HX4 in vitro were measured (Figure 7-2). It can be seen that, although substantially less active than NfsA_Ec, NfsB_Ec is far more active with HX4 than it is with previously tested 2-NI imaging agents such as EF5.
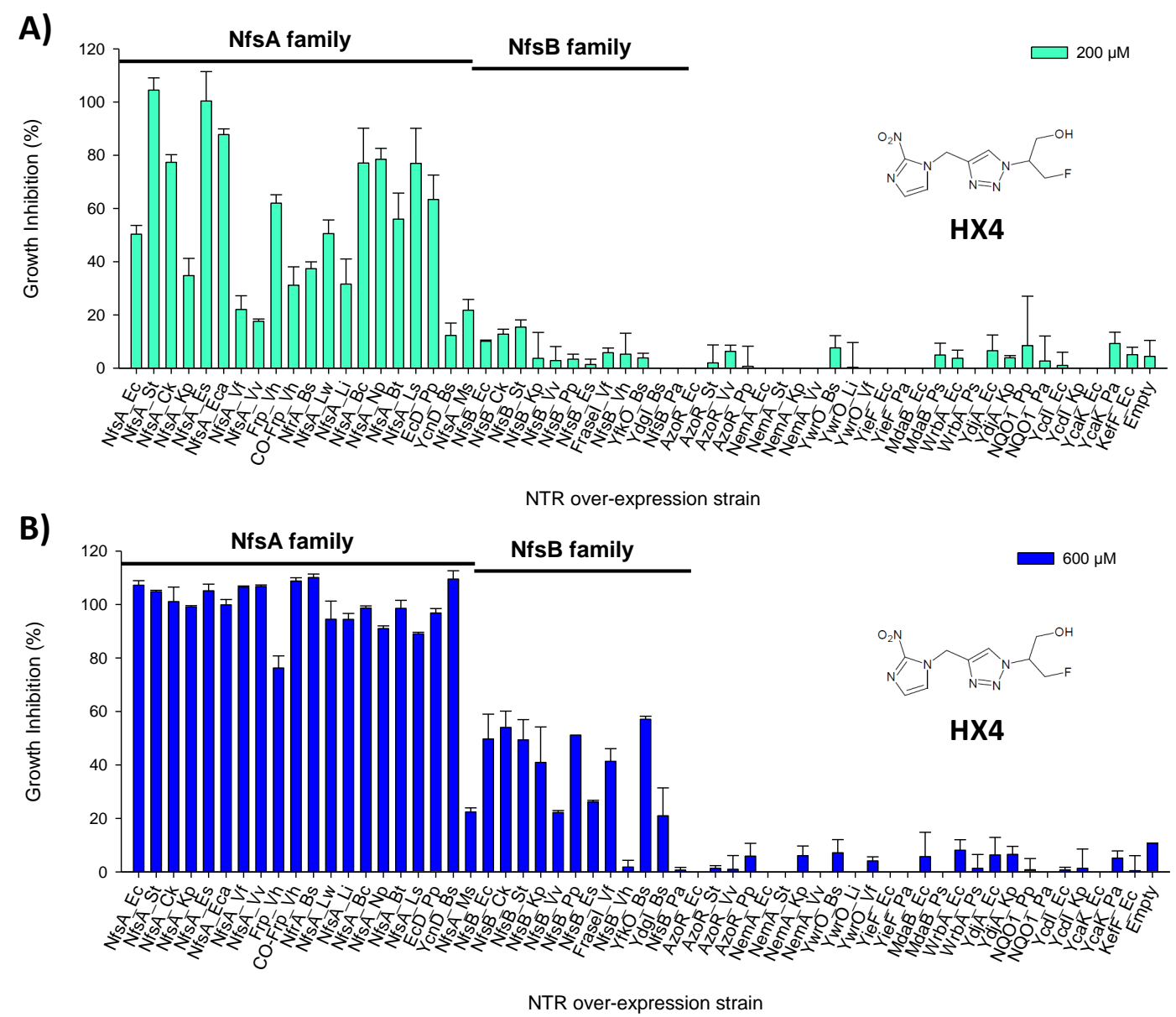

Figure 7-1 HX4 mediated growth inhibition of SOS-R2 E. coli NTR over-expression strains. Mid-exponential phase over-expression strains were challenged in 384 well plate format with HX4 at A) $200 \mu \mathrm{M}$ and B) $600 \mu \mathrm{M}$ for 4 hours. Growth was measured as culture turbidity $\left(\mathrm{OD}_{600}\right)$ pre and post challenge. Growth inhibition is presented as the percentage decrease in the growth of challenged cultures relative to unchallenged controls for each strain. Data are the average of three independent experiments \pm S.E.M. 


\begin{tabular}{|c|c|c|c|c|}
\hline NTR & Compound & $K_{m}(\mu \mathrm{M})^{\mathrm{a}}$ & $\begin{array}{c}k_{\text {cat }} \\
\left(S^{-1}\right)^{a}\end{array}$ & $\begin{array}{c}k_{\text {cat }} / \mathrm{K}_{\mathrm{m}} \\
\left(\mathrm{mM}^{-1} \mathrm{~S}^{-1}\right)\end{array}$ \\
\hline \multirow[t]{2}{*}{ NfsA_Ec } & $\mathrm{EF} 5^{\mathrm{b}}$ & $160 \pm 10$ & $12.1 \pm 0.2$ & 76 \\
\hline & HX4 & $180 \pm 25$ & $13.5 \pm 0.6$ & 75 \\
\hline \multirow[t]{2}{*}{ NfsB_Ec } & $\mathrm{EF} 5^{\mathrm{b}}$ & $1800 \pm 840$ & $0.4 \pm 0.1$ & 0.2 \\
\hline & HX4 & $1120 \pm 160$ & $29.5 \pm 2.4$ & 26 \\
\hline
\end{tabular}

Figure 7-2 Kinetic parameters for the reduction of HX by purified His6-tagged NTRs Rate of reduction at varying concentrations of HX4 and a fixed concentration of excess NADPH $(0.20$ $\mathrm{mM}$ ) were monitored by decrease in absorbance at $340 \mathrm{~nm}$, rates measured in quadruplicate reactions. Apparent $K_{m}$ and $k_{c a t}$ derived using Sigmaplot 10.0 (Systat Software Inc., Richmond, CA). The extinction coefficient used was $19,000 \mathrm{M}^{-1} \mathrm{~cm}^{-1}$ for explanation of determination see section 2.11.1.

\subsubsection{Metabolism of 2-NI imaging agents by NfsA_Ec single mutants showing improved activity with PR-104A}

To assess the effects that the ten identified single mutations favourable for PR-104A activation had on 2-NI metabolism, the $\mathrm{IC}_{50} \mathrm{~S}$ with $\mathrm{HX} 4, \mathrm{EF} 5$ and F-miso were determined for each of the mutant over-expression (SOS-R2) strains (Figure 7-3). Consistent with the results of Chapter 5, the S41Y expression strain was again $\sim$ two fold more sensitised to the 2-NIs than NfsA_Ec wild type. This mutation however conferred the only notable improvement in activity with EF5 and HX4 - the majority of single-residue mutations had little effect or were detrimental, particularly those at site 225 for which up to two fold decreases in drug sensitisation were observed. A more uniform activity profile was seen with F-miso. However, in no case was 2-NI metabolism completely lost (exact compound $\mathrm{IC}_{50}$ values for the SOS-R2 empty plasmid control strain could not be measured due to compound solubility limitations, but are $>5 \mathrm{mM}$; results not shown).

None of the single mutations were found to completely abrogate HX4 activity, offering promise that multi-site mutants selected for improved PR-104A metabolism will retain some ability to metabolise HX4. 


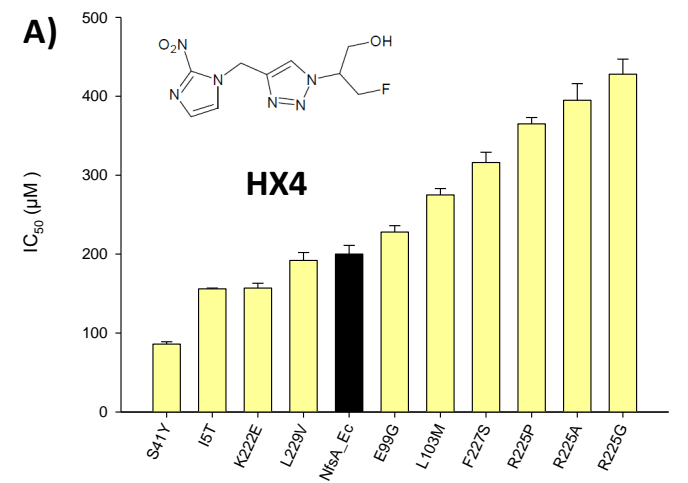

C)

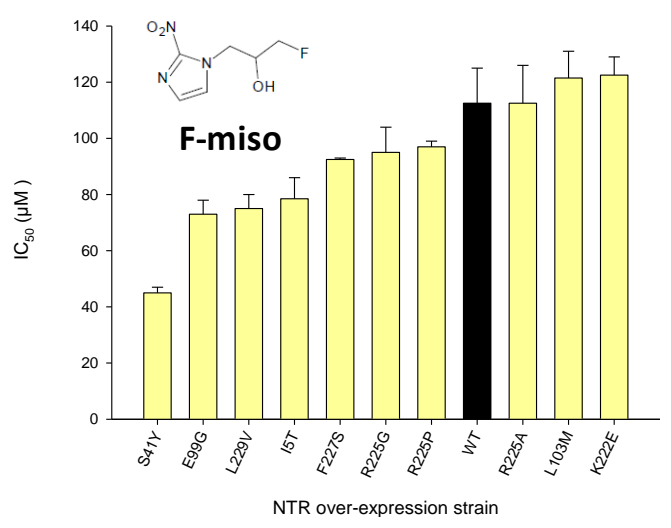

B)

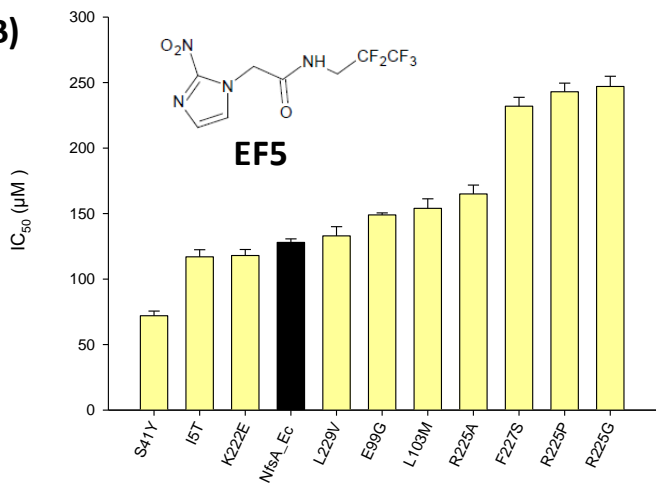

D)

\begin{tabular}{|c|c|c|c|c|c|c|}
\hline & \multicolumn{3}{|c|}{$\mathrm{IC}_{50}(\mu \mathrm{M})$} & \multicolumn{3}{|c|}{ Fold increase in sensitisation ${ }^{1}$} \\
\hline & HX4 & EF5 & F-miso & HX4 & EF5 & F-miso \\
\hline NfsA_Ec & $200 \pm 11$ & $128 \pm 3$ & $113 \pm 13$ & - & - & - \\
\hline I5T & $156 \pm 1$ & $117 \pm 4$ & $79 \pm 8$ & 1.3 & 1.1 & 1.4 \\
\hline S41Y & $86 \pm 3$ & $72 \pm 4$ & $45 \pm 2$ & 2.3 & 1.8 & 2.5 \\
\hline E99G & $228 \pm 8$ & $149 \pm 2$ & $73 \pm 5$ & 0.9 & 0.9 & 1.5 \\
\hline L103M & $275 \pm 8$ & $154 \pm 7$ & $122 \pm 10$ & 0.7 & 0.8 & 0.9 \\
\hline K222E & $157 \pm 6$ & $118 \pm 5$ & $123 \pm 7$ & 1.3 & 1.1 & 0.9 \\
\hline R225A & $395 \pm 21$ & $165 \pm 7$ & $113 \pm 14$ & 0.5 & 0.8 & 1.0 \\
\hline R225G & $428 \pm 19$ & $247 \pm 8$ & $95 \pm 9$ & 0.5 & 0.5 & 1.2 \\
\hline R225P & $365 \pm 8$ & $243 \pm 7$ & $97 \pm 2$ & 0.5 & 0.5 & 1.2 \\
\hline F227S & $316 \pm 13$ & $232 \pm 7$ & $93 \pm 1$ & 0.6 & 0.6 & 1.2 \\
\hline L229V & $192 \pm 10$ & $133 \pm 7$ & $75 \pm 5$ & 1.0 & 1.0 & 1.5 \\
\hline
\end{tabular}

Figure 7-3 2-NI IC $\mathrm{IC}_{50}$ values of SOS-R2 E. coli strains over-expressing NfsA_Ec wild type or single mutants evolved for improved PR-104A metabolism. Drug dependent growth inhibition was monitored by measuring strain turbidity $\left(\mathrm{OD}_{600}\right)$ pre and post four hour incubation with a two-fold dilution series of A) HX4 B) EF5 C) F-miso. Percentage growth relative to unchallenged controls was determined and SigmaPlot ${ }^{\mathrm{TM}} 10.0$ used to calculate the $\mathrm{IC}_{50}$. Graphed strains are ordered by increasing $\mathrm{IC}_{50}$, D) Data shown in table format. Data are the average of three independent experiments \pm SEM.

\subsubsection{Screening of a multi site NfsA_Ec gene library for mutants exhibiting} improved PR-104A metabolism

Generation of an E. coli SOS-R2 pUCX NfsA_Ec multi-site mutant library was accomplished and validated in the similar manner to that described in Chapter 5 - with the one exception being that degenerate mutant genes were ordered pre-synthesised from GenScript rather than being generated by overlap PCR. PCR screening of random colonies enabled the gene insert ratio to be estimated at $85 \%$ therefore a library of $\sim 1900$ clones were screened to give $95 \%$ coverage of the 512 possible combinations of the ten single mutations. Each library plate was first screened with duplicate $10 \mu \mathrm{M}$ PR-104A SOS assays and clones which showed $\mathrm{a} \geq 3$ fold higher SOS response than NfsA_Ec wild type controls were taken forward for repeat screening with SOS assays using 
progressively lowered concentrations of PR-104A (results not shown). Ultimately improved metabolism was confirmed by PR-104A $\mathrm{IC}_{50}$ assay with results for the ten most improved clones shown in Figure 7-4.

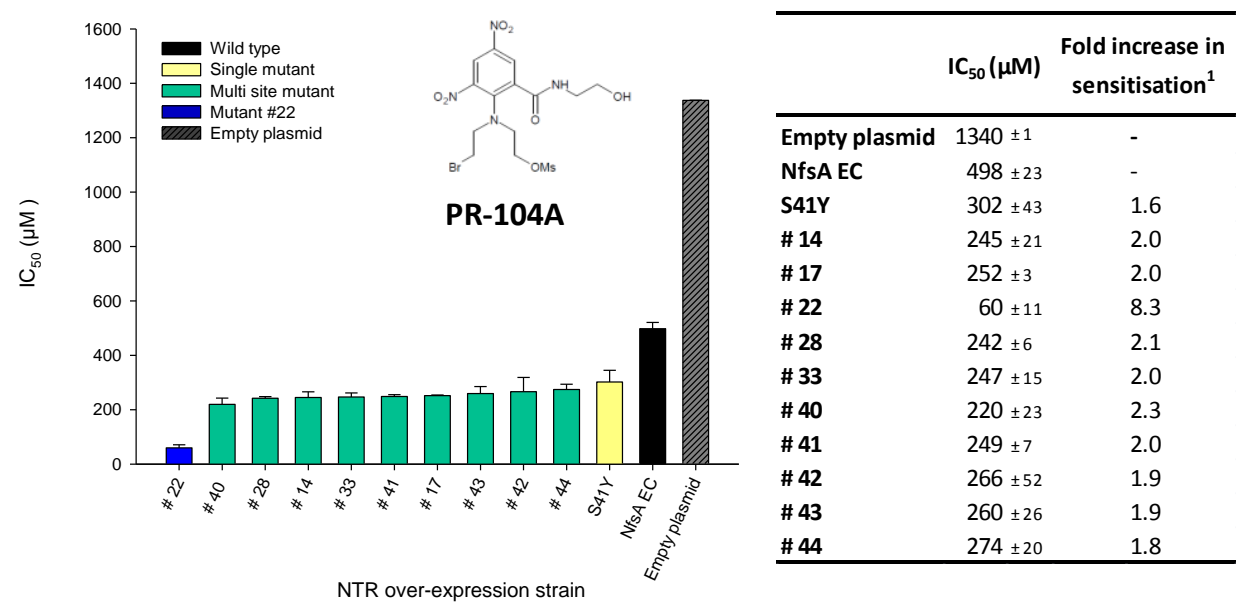

Figure 7-4 PR-104A IC 50 values of SOS-R2 E. coli strains over-expressing mutants identified as showing improved PR-104A activation. $\mathrm{IC}_{50}$ values determined as previously described. Strains are graphed by increasing $\mathrm{IC}_{50}$ and also presented in inset table. Data are the average of three independent experiments \pm SEM. The $\mathrm{IC}_{50}$ value for the SOS-R2 empty plasmid control could be determined at readily achievable concentrations of PR-104A and as such is included. Note: while I aided with construction and initial screening of the multi-site mutant library the experiment in this figure was carried out by Dr. Janine Copp.

The majority of the multi-site mutants only conferred slightly greater PR-104A sensitisation than the single mutation S41Y. The one exception was the clone \#22, which with an $\mathrm{IC}_{50}$ of $60 \mu \mathrm{M}$ showed an 8.3 fold increase in sensitisation compared to NfsA_Ec wild type and a 5.0 fold increase compared to S41Y.

The DNA sequence of each of these ten clones was determined (Table 7-2). All contained two to five mutations. Some mutations were highly represented - for example, all clones contained the S41Y mutation, 8/10 the F227S and 9/10 showed mutations at Arg225. In contrast the mutation L229V did not appear at all, E99G was present only once and K222E twice. 


\subsubsection{2-NI metabolism of multi site NfsA_Ec mutants selected for improved PR-104A activity}

The 2-NI metabolism of these ten multi site mutants was measured by determining IC $_{50}$ values for HX4, EF5 and F-miso (Figure 7-5). Surprisingly (given the number of singleresidue mutations observed to diminish 2-NI activity; Figure 7-3) all multi mutant strains showed an unchanged or greater sensitisation to these compounds than wild type NfsA_Ec.

A)

C)
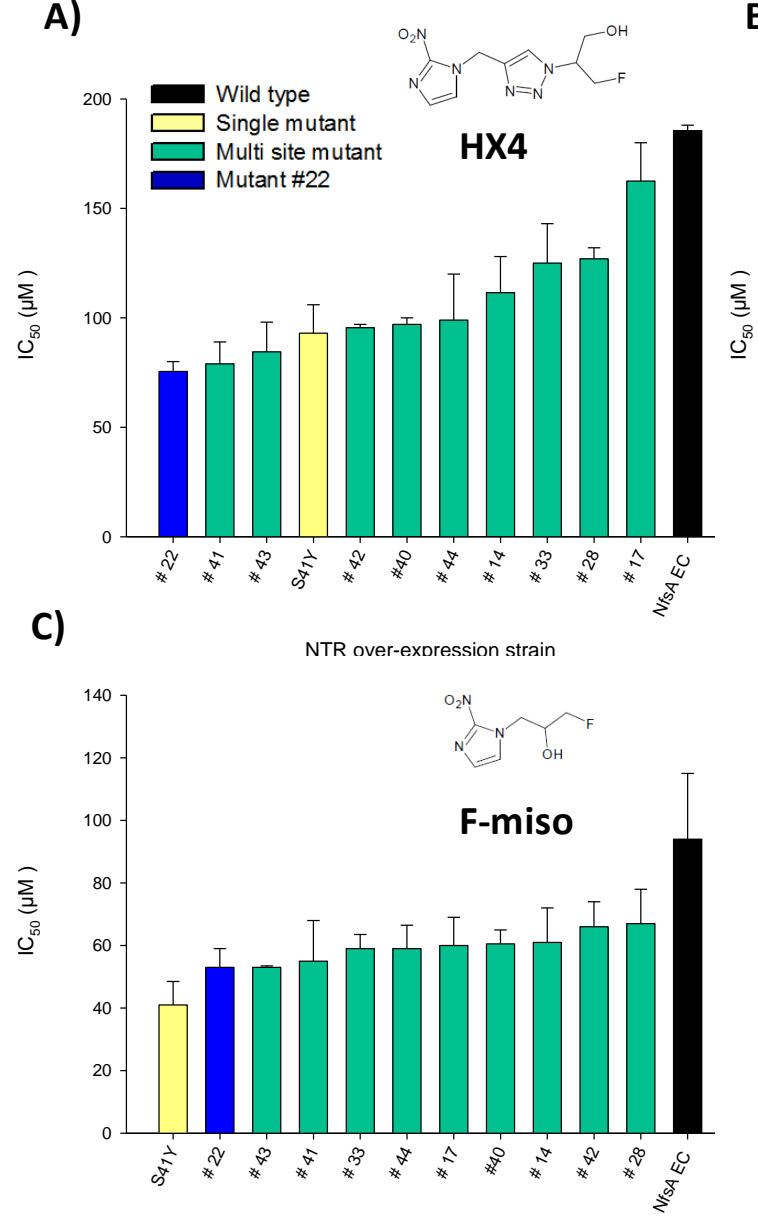

NTR over-expression strain
B)

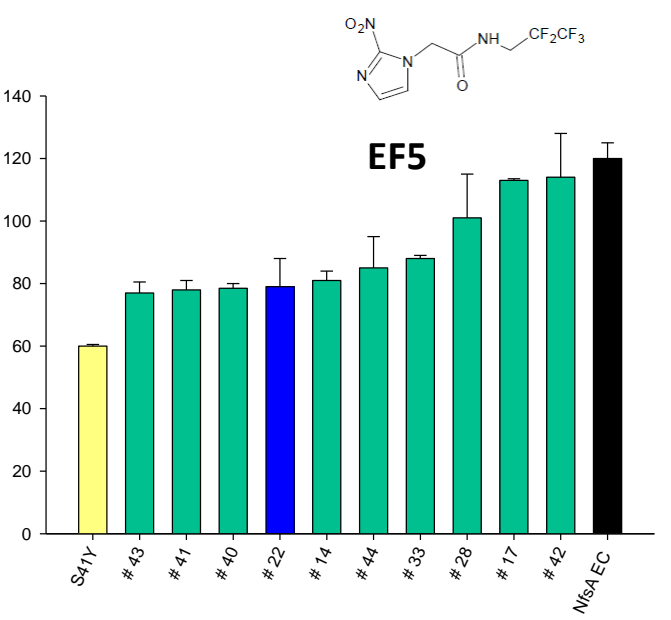

D)

\begin{tabular}{|c|c|c|c|c|c|c|}
\hline & \multicolumn{3}{|c|}{$\mathrm{IC}_{50}(\mu \mathrm{M})$} & \multicolumn{3}{|c|}{${\text { Fold increase in sensitisation }{ }^{1}}^{1}$} \\
\hline & HX4 & EF5 & F-miso & HX4 & EF5 & F-miso \\
\hline NfsA EC & $186 \pm 3$ & $120 \pm 5$ & $94 \pm 21$ & - & - & - \\
\hline S41Y & $93 \pm 13$ & $60 \pm 1$ & $41 \pm 8$ & 2.0 & 2.0 & 2.3 \\
\hline \# 14 & $112 \pm 17$ & $81 \pm 3$ & $61 \pm 11$ & 1.7 & 1.5 & 1.5 \\
\hline \# 17 & $163 \pm 18$ & $113 \pm 1$ & $60 \pm 9$ & 1.1 & 1.1 & 1.6 \\
\hline \# 22 & $76 \pm 5$ & $79 \pm 9$ & $53 \pm 6$ & 2.5 & 1.5 & 1.8 \\
\hline$\# \mathbf{2 8}$ & $127 \pm 5$ & $101 \pm 14$ & $67 \pm 11$ & 1.5 & 1.2 & 1.4 \\
\hline \# 33 & $125 \pm 18$ & $88 \pm 1$ & $59 \pm 5$ & 1.5 & 1.4 & 1.6 \\
\hline$\# \mathbf{4 0}$ & $97 \pm 3$ & $79 \pm 2$ & $61 \pm 5$ & 1.9 & 1.5 & 1.5 \\
\hline$\# 41$ & $79 \pm 10$ & $78 \pm 3$ & $55 \pm 13$ & 2.3 & 1.5 & 1.7 \\
\hline$\# 42$ & $96 \pm 2$ & $114 \pm 14$ & $66 \pm 8$ & 1.9 & 1.1 & 1.4 \\
\hline \# 43 & $85 \pm 14$ & $77 \pm 4$ & $53 \pm 1$ & 2.2 & 1.6 & 1.8 \\
\hline \# 44 & $99 \pm 21$ & $85 \pm 10$ & $59 \pm 8$ & 1.9 & 1.4 & 1.6 \\
\hline
\end{tabular}

Figure 7-5 2-NI IC I0 $_{50}$ values of SOS-R2 E. coli strains over-expressing NfsA_Ec wild type or mutants evolved for improved PR-104A metabolism. IC $_{50}$ values were determined as previously described for A) HX4 B) EF5 C) F-miso. Graphed strains are ordered by increasing $\mathrm{IC}_{50}$. D) $\mathrm{IC}_{50} \mathrm{~s}$ shown in table format. Data are the average of three independent experiments \pm SEM. 


\subsubsection{Metabolism of CB1954 and metronidazole by multi site NfsA_Ec mutants selected for improved PR-104A activity}

The ability of the multi site mutants to metabolise the prototypical prodrug CB1954 was assessed by $\mathrm{IC}_{50}$ assay (Figure 7-6A). The CB1954 sensitisation conferred by the mutants varied widely, with the most sensitised mutant strain (\#17- $87 \mu \mathrm{M})$ having a 15 fold lower $\mathrm{IC}_{50}$ than the least $(\# 22-1320 \mu \mathrm{M})$. Compared to NfsA_Ec wild type only \#17 was improved. Activity with metronidazole, the 5-NI antibiotic intended for use as a biosafety control, was also assessed (Figure 7-6B). Clone \#17 was again improved compared to NfsA_Ec, this time showing a 12 fold lowered $\mathrm{IC}_{50}$ - a fold increase in sensitisation actually larger than that seen for this strain on PR-104A treatment. The other mutants showed more moderate improvements, with the exception of \#22 which had again lost activity. It should be noted that while \#22 strain shows decreased sensitisation to these substrates the NTR is still metabolising them - the SOS-R2 pUCX empty strain has an $\mathrm{IC}_{50}$ of $>5 \mathrm{mM}$ for both metronidazole and CB1954 (results not shown). 

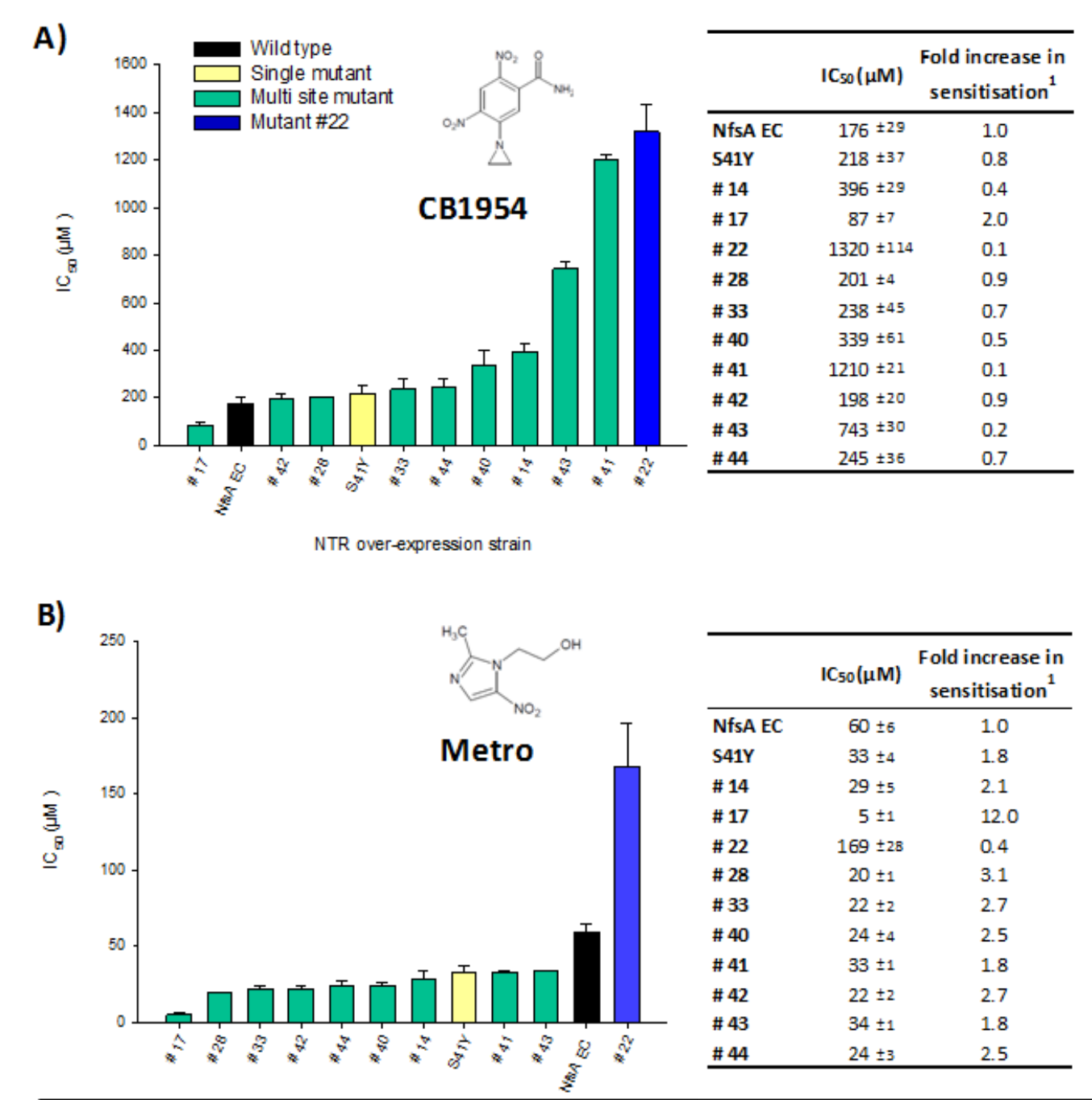

${ }^{1}$ Derived by dividing I $C_{50}$ value on $\mathrm{NfSA}_{5}$ Ec expression by the $I_{50}$ seen on expression of the improved mut ant

Figure 7-6 A) CB1954 and B) metronidazole $\mathrm{IC}_{50}$ values of SOS-R2 E. coli strains overexpressing NfsA_Ec wild type or mutants evolved for improved PR-104A metabolism. IC $_{50}$ values were determined as previously described and graphed in order of increasing $\mathrm{IC}_{50}$. $\mathrm{IC}_{50} \mathrm{~S}$ also shown inset in table format. Data are the average of three independent experiments \pm SEM.

\subsection{Discussion}

Our overall aim with this work is to progress the clinical use of bacterial nitroreductase enzymes in the treatment of cancer. The multi institution collaboration established represents the first time in which the activity of our NTRs will be assessed in a clinically applicable delivery vector - with the added possibility of advancing to clinical trial. Given this opportunity we wished to provide an NTR with the greatest possible activity with our lead prodrug candidate PR-104A. As noninvasive imaging of the recombinant Clostridia by microPET will be attempted we also determined activity of our selected NTRs with HX4 (the PET probe preferred by our collaborators) and the additional 2-NI imaging agents EF5 and F-miso. 


\subsubsection{Selection of the NfsA_Ec variants to be introduced into $C$. sporogenes}

Prior to the work described in this chapter Dr. Janine Copp had identified ten mutations which, when introduced individually into NfsA_Ec, improved metabolism of PR-104A on over-expression in E. coli. To search for multi-site mutants showing further improvement we screened, using PR-104A SOS and $\mathrm{IC}_{50}$ assays, a gene library encompassing sequences with all possible combinations of these ten single mutations. Subsequent to this the ability of the most improved multi-site mutants to sensitise E. coli to 2-NI imaging agents, to the DNA damaging prodrug CB1954, and to the antibiotic metronidazole was determined.

Based on these results NfsA_Ec, S41Y, and the multi-site mutants \#22 and \#17 were selected for testing in $C$. sporogenes and the recombination based introduction of these genes into the bacterium has recently been completed. The NTRs chosen represent the wild type enzyme, both the single (S41Y) and multi-site mutant (\#22) conferring the greatest sensitisation to PR-104A and the multi-site mutant showing the greatest CB1954 and metronidazole sensitisation (\#17).

\subsubsection{MicroPET imaging of Clostridium expressing NfsA_Ec variants}

We are at this point unsure what level of NfsA_Ec activity with ${ }^{18} \mathrm{~F}-\mathrm{HX} 4$ will be required for in vivo microPET imaging of recombinant Clostridia. However it is reassuring that none of the NfsA_Ec multi-site mutants have lost activity with HX4 and many show a moderate improvement in metabolism. This is the case even though these multi-site mutants contain residue changes which, when present singly, were found to be detrimental to 2-NI activity. However the S41Y mutation was found in all multi-site mutants with improved PR1-04A metabolism identified in this chapter and, as S41Y is also the only single mutation which clearly improved HX4 metabolism, this suggests that it is acting as a dominant mutation in regard to 2-NI metabolism. Alongside the mutants presented in this chapter, introduction into C. sporogenes of the mutant 1_56, (which shows improved HX4 metabolism as seen in Chapter 5), has been attempted but as yet these efforts have been unsuccessful. Even if this strain is not completed in the required time-frame for inclusion in microPET ${ }^{18} \mathrm{~F}-\mathrm{HX} 4$ studies, the results from attempted imaging of the remaining $C$. sporogenes-NTR strains will provide valuable data for our long term goal of developing noninvasive imaging of bacterial NTRs. 
In passing it is noted that NfsB_Ec shows a moderate metabolism of $\mathrm{HX} 4$, a feature not seen when this NTR was tested with other 2-NI imaging agents. In subsequent microPET studies it may also be worthwhile to attempt to image NfsB_Ec with ${ }^{18} \mathrm{~F}-\mathrm{HX} 4$. If successful, this could provide a means of leveraging the 20+ years of development of NfsB_Ec as an enzyme for GDEPT, without the need to develop novel chemical matter as per Chapter 6.

\subsubsection{Comparison of the NfsA_Ec variants abilities to metabolise PR-104A and CB1954}

Further examination of the mutant proteins generated during this study might provide useful information about active site confirmations that promote favourable NfsA_Ec-PR104A interactions. While a number of multi-site mutant strains showed improved metabolism of PR-104A, \#22 was substantially more sensitive to PR-104A than all other strains. This apparent increase in metabolism is dependent on the inclusion of the E99G mutation, as can be seen by comparing the activities of \#22 and the otherwise identical \#42 (Figure 7-7). Conversely the changes in the active site brought about by this mutation were found to substantially impede CB1954 metabolism (section 7.4.6).

\begin{tabular}{|c|c|c|c|c|c|c|c|c|c|c|}
\hline & 트 & Уু & $\sum_{\substack{m \\
્}}$ & $\underset{\mathbb{N}}{\stackrel{\mathbb{N}}{\mathbb{N}}}$ & $\underset{\underset{\sim}{\sim}}{\underset{x}{x}}$ & 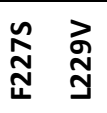 & PR-104A & CB1954 & HX4 & Metro \\
\hline NfsA_EC & & & & & & & - & - & - & - \\
\hline S41Y & $\checkmark$ & & & & & & 1.6 & 0.8 & 2.0 & 1.8 \\
\hline \# 14 & $\checkmark \checkmark$ & & $\checkmark$ & $\checkmark$ & & $\checkmark$ & 2.0 & 0.4 & 1.7 & 2.1 \\
\hline \# 17 & $\checkmark \checkmark$ & & & & $P$ & $\checkmark$ & 2.0 & 2.0 & 1.1 & 12.0 \\
\hline \# 22 & $\checkmark$ & $\checkmark$ & $\checkmark$ & & G & $\checkmark$ & 8.3 & 0.1 & 2.5 & 0.4 \\
\hline \# 28 & $\checkmark \checkmark$ & & $\checkmark$ & & G & $\checkmark$ & 2.1 & 0.9 & 1.5 & 3.1 \\
\hline \# 33 & $\checkmark$ & & $\checkmark$ & & A & $\checkmark$ & 2.0 & 0.7 & 1.5 & 2.7 \\
\hline$\# 40$ & $\checkmark \checkmark$ & & & $\checkmark$ & A & & 2.3 & 0.5 & 1.9 & 2.5 \\
\hline \# 41 & $\checkmark$ & & & & A & $\checkmark$ & 2.9 & 0.1 & 2.3 & 1.8 \\
\hline \# 42 & $\checkmark$ & & $\checkmark$ & & G & $\checkmark$ & 1.9 & 0.9 & 1.9 & 2.7 \\
\hline \# 43 & $\checkmark \checkmark$ & & & & A & $\checkmark$ & 1.9 & 0.2 & 2.2 & 1.8 \\
\hline \# 44 & $\checkmark$ & & & & A & & 1.8 & 0.7 & 1.9 & 2.5 \\
\hline
\end{tabular}

Figure 7-7 Heat map representation of activities of NfsA_Ec multi site mutants with DNA damaging prodrugs PR-104A and CB1954, 2-NI imaging agent HX4 and 5-NI antibiotic metronidazole. Individual mutations present in each protein are indicated to the left. Data shown to the right is fold increase in sensitisation, compared to NfsA_Ec wild type, derived from dividing compound $\mathrm{IC}_{50}$ value for NfsA_Ec wild type over-expression strain by the $\mathrm{IC}_{50}$ seen on mutant over-expression. Values colour coded in activity groups of $0.1-0.8,0.9-1.1,1.2-2.0,2.1$ $-4.0,>4.0$. 
The entirely differing effects of the various mutations in promoting CB1954 vs. PR-104A metabolism suggest that, even though the two drugs share a common dinitrobenzamide core, their requirements for favourable active site binding are very different. A solved Xray crystal structure of NfsA_Ec variant \#22 would be highly beneficial to elucidating the different binding modes of PR-104A and CB1954 in the multi-site mutant binding modes and would provide information for further evolution studies. However, although efforts to crystallise \#22 have been initiated, these efforts have as yet been unsuccessful (Christopher Squire, personal communication). 


\section{Chapter 8: Key findings, conclusions and future directions}

\subsection{Research motivation}

The research described in this thesis was part of a multidisciplinary collaboration aimed at developing nitroreductase GDEPT, an experimental targeted anti-cancer treatment. My project focused on screening and engineering bacterial NTRs with a long-term aim of developing the capability to noninvasively image the location and levels of therapeutic NTR expression in a clinical setting. Notable clinical success of the NTR/GDEPT strategy has yet to be demonstrated but previous research has predominantly used the enzyme/prodrug pair of NfsB from E. coli and the nitroaromatic prodrug CB1954. In contrast, we and our close collaborators at the ACSRC aim to improve this treatment by the use of more potent prodrugs - most immediately, the dinitrobenzamide mustard PR104. In parallel we have sought to identify superior enzymes for GDEPT by using E. coli over-expression libraries to assay for NTRs better able to activate prodrugs and PET probes. Reduction of these probes could enable PET based noninvasive imaging of NTR expression. It is well recognised that this capability would be extremely advantageous to preclinical and clinical development of NTR cancer gene therapy (Baril et al., 2010; Bhaumik et al., 2011; Rojas and Thorne, 2012) but prior to this work no such ability for bacterial NTRs has been described.

\subsection{Key findings}

While no PET suitable reporter probes have previously been described for bacterial NTRs, there do exist a number of 2-NI PET probes developed independently to enable clinical imaging of hypoxia. In the first chapter of this thesis it was shown that, of 11 diverse E. coli oxidoreductase enzymes, only NfsA could reduce the 2-NI hypoxia imaging agents pimonidazole, F-miso, EF3 and EF5. In contrast NfsB_Ec showed little to no ability to reduce 2-NI compounds. NfsA_Ec activity was detected in screens monitoring sensitisation of the E. coli over-expression host to 2-NI compounds, as well as a colourimetric assay monitoring 2-NI activity dependent depletion of NADPH by E. coli cell lysates. Importantly our collaborators went on to show that expression of NfsA_Ec in human cells can be detected in vitro and in vivo by using antibodies to detect the NfsA_Ec catalysed binding of EF5. 
When a larger collection of NTR candidates sourced from different bacteria was examined, 2-NI reduction was found to generally be a property of only the NfsA homologues, although some more distantly related NfsB_Ec homologues showed a moderate ability to metabolise F-miso and HX4. Surprisingly, the activities of individual $\mathrm{NfsAs}$ as measured by either growth inhibition $\left(\mathrm{IC}_{50}\right)$ or NADPH depletion assays showed little correlation. Evaluation of kinetic parameters for the reduction of EF5 by a number of purified NfsA enzymes suggested that this discrepancy may be due to each assay emphasising a different parameter of enzymatic activity, i.e. the results of the NADPH depletion assay showed good correlation with the enzymes' apparent $k_{\text {cat }}$ but not the apparent $K_{m}$; while the opposite scenario was observed for the growth inhibition assays.

To improve NTR activation of 2-NI PET probes, NfsA_Ec was selected for directed evolution by site saturation mutagenesis, targeting 12 residues surrounding the NfsA_Ec active site. In total, 31 different single mutations were identified that improved F-miso and/or EF5 metabolism. Ten of these were combined in a degenerate gene library and screened to recover variants containing additive or synergistic combinations of these mutations. Eight individual multi-mutant sequences were identified that, when expressed in E. coli, conferred a 3.2 - 4.8 fold increased sensitisation to EF5, F-miso, and HX4 compared to NfsA_Ec wild type. The triple mutation motif S41X/L103V/N134A (where $\mathrm{X}$ indicates either Cys or Tyr) was strongly represented in these eight variants. From a model of the S41Y single mutation variant and preliminary $\mathrm{x}$-ray crystallography data generated by a collaborator it appears that the S41Y substitution may favour binding of aromatic substrates by promoting stacking of the substrate between the FMN cofactor and the aromatic side chain of tyrosine. Disappointingly, no improvements in EF5 metabolism by HCT-116 cells expressing any of these variants were observed relative to cells expressing wild type NfsA_Ec, although decreased expression of the mutant NfsA_Ec variants and/or technical limitations of the assay may have contributed to this outcome.

As an alternative strategy to improve the sensitivity of NTR PET imaging, our collaborators designed and synthesised a 5-NI analogue of EF5, named SN33623. Experiments to date (with my involvement) have indicated that SN33623 is substantially less responsive to hypoxia than EF5. Screening of a wide range of NTR candidates revealed that a subset of $\mathrm{NfsB}$ enzymes could readily metabolise this compound but that this subset did not include NfsB_Ec. Sequence alignment and site-directed mutagenesis 
were then used to ultimately identify two key mutations (F70A and F108Y) that can be introduced into either $\mathrm{NfsB}$ _Ec wild type or previously evolved NfsB_Ec variants to confer high levels of SN33623 activity. These variants were also shown to metabolise SN33623 (unlike wild type NfsB_Ec) when expressed in HCT-116, though for some variants this activity was only evident immediately following NTR transfection, with a loss of function observed following long term expression from HCT-116.

In a final aspect of this study a collaborative directed evolution study was conducted to improve PR-104A metabolism by NfsA_Ec while retaining 2-NI metabolism. SOS assay screening of a multi-site NfsA_Ec mutant library was conducted to identify variants with improved PR-104A metabolism, followed by counter-screening for metabolism of other clinically relevant nitroaromatic compounds. Importantly, all mutants were found to retain 2-NI metabolism, most likely due to the presence of a dominant S41Y mutation in all selected variants. On the basis of activity with (primarily) PR-104A and (secondarily) HX4, CB1954 and metronidazole, four promising NfsA_Ec variants were chosen for future evaluation in $C$. sporogenes, targeting human clinical trials.

\subsection{Critical evaluation of the $E$. coli screens used in this study}

Laboratory strains of E. coli are well studied, fast to grow and highly tractable to genetic modification. These and other features underpin the widespread use of E. coli as a protein expression system and screening tool in directed evolution studies. However, a critical question for this study is whether this screening of NTR catalysed 2-NI growth inhibition in $E$. coli is genuinely predictive of the ability of an NTR to act as a reporter gene in a preclinical or clinical context. At the broadest level, it appears that E. coli growth inhibition can accurately predict complete absence or presence of imaging agent metabolism by HCT-116:ntr strains. This approach accurately identified NfsA_Ec as the only one of 11 diverse enzymes capable of metabolising EF5 and also predicted the ability of strains of HCT-116:NfsB_Ec and various NfsB_Ec variants to metabolise SN33623 (albeit in some cases only upon transient NTR expression). However, we do not know the extent to which the E. coli based screening successfully reports on incremental degrees of activities with either imaging agents or prodrugs, nor to what extent improvements in NTR catalytic activity measured using these screens will carry over to a clinical setting - critical points for evaluating the use of E. coli for directed evolution to improve such activities. 


\subsubsection{Variations in bacterial NTR expression levels from human cells}

Our collaboration had aimed to assess the validity of the E. coli based screens in vitro and in vivo preclinical models of stably transfected HCT-116 cell lines, however accurate determination of bacterial NTR activity was confounded by the unpredictable and varying levels of functional NTR expression observed. Particularly problematic was that the expression and/or activity of some NTRs was found to diminish over time.

Reasons for the heterogeneous expression of different NTRs, introduced in some cases by a single amino acid change, are unknown. One possibility is that the NTRs showing problematic expression catalyse metabolic activities that are cytotoxic to human cells, such that functional expression of these NTRs is selected against over time. This hypothesis could be tested by PCR amplifying and sequencing F279-V5:ntr plasmids from cell lines that have lost functional NTR expression over several weeks in culture, to determine if the NTR or plasmid regions have developed mutations that are diminishing expression of functional NTRs. Investigating whether certain NTRs are indeed perturbing HCT-116 metabolism and if so, what cellular processes they are interfering with, would be a much more extensive project; but could perhaps be attempted by metabolomic studies in which chromatography-mass spectrometry technologies were applied to compare the metabolite profiles from wild type HCT-116 versus cells expressing different NTRs that are tolerated to various degrees (Bennett et al., 2009). It should be noted that other explanations are also possible, for example some unidentified protein level instability for which protein thermostability is a poor surrogate measure, or that the observed variable NTR expression is a consequence of multiple different factors (which could complicate any study to determine the exact underlying reason(s) for this issue).

It is important to reiterate that most vectors for GDEPT would not be expected to mimic a stable transfection / expression scenario, and that our findings emphasise the need to evaluate selected NTRs in the context of their intended clinical vector. Our collaboration will in the near future be able to assess the expression and activity of a number of NfsA_Ec variants in Clostridium, and we hope that this bacterial vector will allow for NTR expression more similar to that seen from E. coli rather than the problematic HCT116 expression. In the future we hope to also progress testing of a selection of NTRs in different viral vectors, either through developing further collaborations or through use of viruses which are already available to us (e.g. ONYX-411; Singleton et al., 2007). Assessing functional NTR expression across a range of the most clinically relevant 
vectors will enable us to evaluate the validity of our $E$. coli based directed evolution and to determine if the currently observed variable NTR expression levels are indicative of a greater problem.

\subsubsection{Purified protein activities of evolved NTR variants}

To assess whether our mutant NTRs generated via $E$. coli based directed evolution studies were truly improved in ability to catalyse the targeted reactions, we examined activity at a purified protein level - however, our results were not clear cut. In multiple different directed evolution projects conducted in our laboratory (FRaseI activation of CB1954 (Swe et al., 2012) and NfsA_Ec activation of EF5 or PR-104A (this study)) many NTR variants selected for increased ability to sensitise E. coli to these compounds counterintuitively had, when tested as purified proteins, decreased specificity constants for the respective reactions. The apparent $K_{m}$ values for the selected mutants were near universally decreased, consistent with improved enzymatic activity; but because the apparent $k_{\text {cat }}$ values were also decreased (in some cases dramatically) this frequently led to a net reduction in specificity constant. As previously discussed, the specificity constant $k_{\text {cat }} / K_{m}$ can be a poor measure of the relative activities of different enzymes, and these kinetic parameters could indicate that the mutants have improved activities with the target substrates at very low concentrations, with discovery of such mutants having been favoured in the SOS assay. However an alternative possibility is that the mutants confer increased in vivo sensitisation to these compounds because they have lost ability to metabolise endogenous $E$. coli substrates that perhaps otherwise compete with the prodrug/imaging agent for the NTR active site. This possibility is not without precedent, for example the role that competing thymidine metabolism has on modulating HSV1-TK phosphorylation of xenobiotic prodrugs and imaging agents is well recognised (Gambhir et al., 2000; Kokoris and Black, 2002). In one example, a mutant of HSV1-TK that conferred greater in vivo GCV sensitisation than wild type actually had (at a purified protein level) a 40 fold decrease in specificity constant for GCV (with both an increased $K_{m}$ and a decreased $k_{\text {cat }}$ ) but also a 3000 fold lowered specificity constant with the competing thymidine substrate (Kokoris et al., 1999).

Deconvolution of the effects that competing endogenous metabolites may have on activity of NTRs expressed in E. coli would be difficult as, in contrast to the identified thymidine substrate of HSV1-TK, the exact identity of potential competing endogenous substrate(s) is unknown and NTRs are known to be promiscuous enzymes (Gonzalez et 
al., 2005; Roldán et al., 2008; Zenno et al., 1996). Quinones are obvious candidates and we could possibly assess the relative abilities of purified NfsA_Ec variants and NfsA_Ec wild type to reduce a number of commercially available quinones that are known to naturally occur in E. coli (Shestopalov et al., 1997). However such a strategy is not ideal, as negative results would not rule out the competing metabolite theory but could simply indicate that we were not testing the major competing substrate(s). An alternative strategy could be to investigate whether it is possible to inhibit the ability of purified wild type NfsA_Ec to reduce PR-104A (a substrate whose reduction can be directly monitored for by light absorbance at $400 \mathrm{~nm}$ ) by co-incubating this reaction mix with E. coli cell lysate. If cell lysate is able to act as a competitive inhibitor of purified NfsA_Ec reduction of PR$104 \mathrm{~A}$ it should then be possible to determine if cell lysate is less able to inhibit the NfsA_Ec variants that slowly reduce PR-104A in vitro but show high PR-104A metabolism on expression in E. coli.

If increases in NTR metabolism of prodrug/imaging agents are being driven not by direct improvements to the reaction, but by a reduction in the ability to metabolise competing substrates, then this could greatly complicate the use of $E$. coli based screening for directed evolution studies as "improved" catalytic activity would heavily dependent on the chemical milieu of the cell in which the NTR is expressed and this could vary widely and unpredictably between $E$. coli and various cancerous cellular environments. This may prove to be less problematic for therapies in which the NTR is expressed from a bacterial vector such as Clostridium or Salmonella as these may show a more similar chemical environment to $E$. coli. Our ongoing trials in $C$. sporogenes may shed some light on this issue.

If in these or other studies it becomes apparent that our "improved" variants are actually less efficient than the wild type enzyme, i.e. that the greatly reduced apparent $k_{c a t}$ values for prodrug/imaging agent reduction do lead to reduced NTR activity in therapeutically relevant expression systems, it may be worthwhile to use the NADPH depletion assay as a screen for directed evolution projects. In this screen the NTR would be exposed to significantly more prodrug/imaging agent than to cellular (potentially competing) metabolites, which should reduce the ability of these metabolites to impact on prodrug/imaging agent reduction. 


\subsubsection{Additional points to consider in assessment of the kinetic assays}

Another possibility that should be briefly investigated is if the NfsA_Ec variants showing low apparent $k_{\text {cat }}$ values for prodrug/imaging agent reduction are being evaluated in a fully active form. NfsA_Ec is active as a homodimer, and it may be possible that some of the NTR mutations are weakening the dimeric stability. It has been reported that in Frp_Vh (a NfsA homologue with strong structural similarity to NfsA_Ec (Kobori et al., 2001)) substituting the Glu99 residue for Lys leads to extreme dimer destabilisation and greatly lowered in vitro activity (Jawanda et al., 2008). This hypothesis would require that the effects of dimer destabilisation primarily occur in the in vitro (and not in vivo) setting. While I do not consider this to be particularly likely, I note that Glu99 is one of the NfsA_Ec sites at which mutation led to a markedly slower $k_{c a t}$ value for PR-104A reduction, and the mutated residue Arg255 also sits right at the dimer interface, suggesting that it may yet be worthwhile assessing the dimer-monomer equilibriums of a sample of NfsA_Ec variants by size exclusion chromatography (Jawanda et al., 2008).

Finally it should be noted that we have not investigated any potential effects that mutations may have had on the NTR interactions with the electron donors NADPH and NADH. All purified protein reactions were carried out at high (presumably well in excess) concentrations of NADPH, and activities over a range of $\mathrm{NAD}(\mathrm{P}) \mathrm{H}$ concentrations could be assessed to determine if changes to these parameters could explain the relative activities of the NfsA_Ec variants in vivo.

\subsubsection{Summary}

We cannot draw strong conclusions about the validity of our E. coli based directed evolution screening without having tested these NTRs in preclinical models that better approximate the desired therapeutic application of these enzymes. We cannot currently rule out that activity of our improved NTR variants may be context-dependent, modulated by competing $E$. coli cell metabolites. We also do not yet know if variable NTR expression from clinically relevant vectors will occur. It should be noted however that others using E. coli to screen for improved NfsB_Ec mutants have recovered variants that increase prodrug-mediated cytotoxicity in human cells (Grove et al., 2003; Guise et al., 2007; Jaberipour et al., 2010). Most relevant to this study are the two NfsB_Ec mutants (T411/N71S and T41L/F70A) generated by Peter Searle's research group, which on NTR delivery to SKOV13 cancer cells (via a replication defective adenoviral vector) provided significantly better CB1954 sensitisation than NfsB_Ec wild type both in vitro and in vivo 
(Jaberipour et al., 2010). It is reassuring for the validity of our own E. coli based screening systems that these improved mutants, when tested in this study, yielded a lower E. coli CB1954 IC $_{50}$ relative to NfsB_Ec wild-type. However consideration should be given to the fact that these particular mutants were identified through an E. coli screen using high (lethal) concentrations of CB1954 (in comparison to the low sub lethal concentrations used in the SOS assay) and reportedly possess a decreased CB1954 $K_{m}$, but similar $k_{\text {cat, }}$ to wild type NfsB_Ec (Jarrom et al., 2009).

\subsection{Further possible directed evolution studies}

Should the testing of NTR activity in clinically relevant vectors indicate that overall our current E. coli based screening strategies are valid, but that further improvement in imaging agent metabolism would be desirable, there would be a number of ways to pursue this. This thesis presented initial work testing the feasibility of screening large libraries for 2-NI improvement using a FACS based enrichment strategy. While some enrichment for active clones was seen, future use of this technique would depend on an exact determination of the enrichment ratio, how consistent this is and whether it can be improved. Firstly we will need to conclusively determine whether the cells that we want to select (i.e. those that are expressing active NTRs and therefore experiencing the highest levels of DNA damage and GFP upregulation), are less able to survive the FACS process than those expressing inactive NTRs. The most straightforward way of doing this would be to compare colony survival ratios of NfsA_Ec populations which have been exposed to titrated RB6145 concentrations and then subjected to FACS sorting and collection based on an arbitrary gate with no GFP selection pressure. If a detrimental effect is seen at desirable RB6145 concentrations, it should next be assessed if PCR rescue is able to efficiently rescue the DNA sequences of highly GFP expressing cells. Also worthwhile would be examining if the currently observed 5-fold enrichment for GFP positive cells changes if initial starting concentrations of GFP positive versus GFP negative cells are lower (e.g. a 1:99, or 1:999 positive to negative ratio, rather than the 1:9 previously tested), as low concentrations of GFP positive cells are more likely to reflect the situation in mutant libraries.

A parallel or alternative approach would be to build on the increased 2-NI metabolism already shown by the multi-site mutants such as S41X/L103V/N134A. This could be done by using these gene sequences as templates for site saturation mutagenesis of other 
active site residues, in particular targeting residues such as Phe42, Cys45 or Phe83 at which multiple amino acid changes were previously observed to improve NfsA_Ec wild type activity. Depending on the success of the FACS approach we could also pursue improving these mutants further by mutations outside of the active site through screening of random mutagenesis libraries generated by error-prone PCR.

\subsection{Research applications}

\subsubsection{The use of diverse NTRs in GDEPT}

Our discovery of novel wild type and engineered NTRs for GDEPT applications removes the requirement for all prodrugs or imaging agents to be efficient substrates of NfsB_Ec. Appropriate chemical selection for a GDEPT therapy already requires simultaneous consideration of many important factors such as previous clinical testing, potential offtarget toxicity, bystander potential, and pharmacokinetic availability. Essentially drug design for GDEPT requires balancing many potentially competing features and it would be advantageous to be able to focus solely on optimising the chemical criteria, without having to also add on the additional selection pressure of picking the best substrate for a single enzyme target. A particularly apparent example of the benefit of screening a wide range of NTRs is that NfsB_Ec does not reduce any of the clinical 2-NI imaging agents (EF5, EF3, F-miso and Pimo) which we intially assessed it against.

It should be noted that mutant NTRs arising from 2-NI evolution studies will not necessarily prove optimal for enzyme prodrug therapy, as residue changes that aid 2-NI metabolism will not necessarily improve prodrug metabolism and could quite possibly impede it. Nonetheless, it is promising that the NfsA_Ec multi-site mutants selected in this study for improved 2-NI metabolism retained or slightly improved PR-104A metabolism (Chapter 5), and vice versa for variants selected primarily for improved PR104A reduction (Chapter 7). 2-NI directed evolution efforts may also provide valuable information about how to engineer in imaging agent activation to NTRs with high prodrug activity, analogous to the manner in which SN33623 metabolism was engineered into CB1954 improved NfsB_Ec variants (Chapter 6). Additionally, 2-NI evolved NTRs could also be useful in preclinical development of GDEPT as testing their 2-NI metabolism in a microPET setting would enable us to determine the threshold level of bacterial NTR/2-NI activity required for accurate and sensitive PET imaging. 


\subsubsection{Noninvasive PET imaging of NTR based therapy}

Collaborators at the University of Maastricht are in the very early stages of assessing whether tumour xenografts stably expressing NfsA_Ec can be noninvasively imaged using ${ }^{18} \mathrm{~F}-\mathrm{HX} 4$ microPET. This will provide a valuable initial indication of whether 2-NI hypoxia imaging agents can be successfully repurposed as noninvasive reporter probes to detect NTR-labelled cells. In a more clinically relevant context, another series of experiments will also be carried out to determine if a Clostridium tumour seeking strain expressing NfsA_Ec variants can also be tracked in vivo using ${ }^{18}$ F-HX4. Studies of these Clostridium strains will also assess the tumour response to prodrugs such as PR-104A. We are not yet certain that the NTRs we have generated from this directed evolution work are truly improved in the tumour environment, and on this point the intended inclusion in trials of Clostridium expressing NfsA_Ec wild type (which shows good purified protein activity and stable functional expression from HCT-116) is reassuring. An additional point of interest will be whether endogenous Clostridium NTRs are also able to reduce ${ }^{18} \mathrm{~F}-\mathrm{HX} 4$ to a cell entrapped form. C. sporogenes has an NfsA_Ec homologue (19\% amino acid identity and 34\% similarity identified via blastP search) and C. sporogenes are sensitive to the 2-NI anti-parasitic prodrug benznidazole (Hof, 1989), which suggests that there is potential for some level of endogenous 2-NI reduction to occur. If ${ }^{18} \mathrm{~F}-\mathrm{HX} 4$ reduction due to $C$. sporogenes NTRs does occur to a level sufficient to be detected by PET, this could actually prove beneficial for the in vivo tracking of the Clostridium as it may increase the total ${ }^{18} \mathrm{~F}-\mathrm{HX} 4$ Clostridium signal.

\subsubsection{The potential use of bacterial NTRs as vector reporter genes independent of GDEPT}

At this very early point in assessment of NTR/2-NI microPET imaging we cannot make predictions about the possible efficacy of this reporter gene/probe pair as compared to the clinically tested hNIS/ ${ }^{99 \mathrm{~m}}$ Tc SPECT and HSV1-TK based PET imaging. However if it does prove to offer at least comparable sensitivity and accuracy to existing modalities, there are reasons to think there would be a place for this reporter/gene pair for imaging in a wider context. For example, it is likely that the addition of HSV1-tk for imaging purposes would be inappropriate for certain specific oncolytic viruses such as JX-594 (Parato et al., 2011) and others in various stages of clinical and preclinical development (Haddad et al., 2012; McCart et al., 2004; Thorne et al., 2007; Zhang et al., 2007) where a portion of the viral tumour specificity is due to the deletion of its endogenous thymidine 
kinase. In regards to hNIS, its endogenous expression in the stomach, bladder and thyroid means hNIS is not suited as a reporter gene for vectors directed towards these areas (Barton et al., 2003). In addition NTRs ( 700 bp) are nearly four times smaller than hNIS (Barton et al., 2003), which could make NTRs more appropriate for insertion into the backbones of viruses with defined packaging limits, particularly in situations where some of the viral insert capacity is already taken up by expression of other therapeutic genes or reporter genes for optical imaging (Penheiter et al., 2012).

\subsection{Concluding remarks}

It is promising for our long-term goal of developing NTR based GDEPT that the NfsA family can efficiently metabolise PET-suitable imaging agents already in clinical use, and in the very near future our multi-disciplinary collaboration will be able to assess if this activity is sufficient to allow noninvasive microPET imaging of NfsA_Ec expression. Furthermore this thesis demonstrates that with enzyme engineering techniques and E. coli based screening we can successfully increase the abilities of NTRs to sensitise their $E$. coli over-expression host to multiple imaging agents and prodrugs. Determining the wider validity of our E. coli based screens has been challenging, but the recently completed generation of $C$. sporogenes spores expressing our NTRs will soon allow for an exciting assessment of both the therapeutic efficacy and noninvasive imaging capabilities of a number of our NfsA_Ec variants in a clinically relevant bacterial vector model. 


\section{Appendix 1:}

\section{$\underline{\text { NfsA_Ck }}$}

MTPTIDLIRGHRSIRHFTDEPISDAQRESIIAAARGTSSSSFLQCSSIIRITDKAMREALVPLTGGQKHV AQAAEFWVFCADFNRHLQICPDAQLGLAEQLLLGTVDTAMMGQNALTAAESLGLGGVYIGGIRN HIEAVTERLKLPKYVLPLFGLCLGWPADNPGVKPRLPAELVVHENHYQPVDAALLAQYDEQIAEY YLTRDSNTRRDTWSDHIRRTIIKENRPFILDYLHKQGWATR

\section{NfsA_Kp}

MTPTIELLRSHRSIRHFTDAPVSDEQRAEIIASAQAASTSSFLQCTSIIRITDPALRERLVPLTGGQQHV AQAAEFWVFCADFNRHLQICPQAQLGLAEQLLIGVVDTALLAQNALTAAESLGLGGVYIGGLRNSI EAVTELLELPQHVLPLFGLCLGWPADNPDIKPRMPAAMLVHENRYQPLDNALLAEYDEQLAHYY LSRGSNARRDTWSDHIRRTIVKESRPFILDYLHKQGWATR

\section{$\underline{\text { NfsA_Eca }}$}

MIPTIDLLQRHRSIRAFTSQAVTDEQRHAIIASAQSASSSSFLQCSSIIRITDPAVRETLIHYTGEQAYV AQAAEFWVFCADFHRHVEIFPQAETGLAEQLLIGCVDTAIMAQNALVAAESLGLGGVFIGGIRNRI ADVTQLLQLPTLVIPLFGLCLGHPDAEPQLKPRMPTAMMLHENVYQPLDRDVLAQYDQQMVEYY LQRTGSRRESWSEHVELTLKKELRPFMLDYLHQQGWAIR

\section{$\underline{\text { NfsA Vf }}$}

MNPVIDTILEHRSIRSFTNEPISKEQLDTIISAGIAASSSSLLQVNSIIRITDKEKRKALVELSGGQPYVE GAAEFLVFCIDFQRHYEMNPEIKAEFTELTLIGAVDAGIMAQNCLLAAESMGLGGVYIGGLRTNAQ GVDDLLELPKNTAVLFGMCLGYPNQAPQKKPRLSPDVIVHENSYQPLDKSKIDEYDEIMQSYYAT RSTNQKQSSWSEQITGKLSQESRPHIKGYLNNKGLAIK

\section{$\underline{\text { NfsA Vv }}$}

MNAVIDTLLSHRSIRKFTDQAITPEQLDTIIRAGLAASSSSLLQVVSIIRITDPAKRQQLAELAGPQHY VETAAEFLVFCIDYQRHATLNSEVQAGFTELTLIGAVDAGIMAQNCLLAAESMGLGGVYIGGLRN KAAEVDALLELPPFSAVLFGMCLGHPDQDPDLKPRLPAEVILHENHYQPLDLNKVEQYDQTMLDY YGKRSSNQKQASWSEQVTGKLAGESRPHILPYLHSKGLATK

\section{$\underline{\text { Frp_Vh }}$}

MNNTIETILAHRSIRKFTAVPITDEQRQTIIQAGLAASSSSMLQVVSIVRVTDSEKRKQLAQFAGNQA YIESAAEFLVFCIDYQRHATINPDVQADFTELTLIGAVDSGIMAQNCLLAAESMGLGGVYIGGLRNS AAQVDKLLGLPENSAVLFGMCLGHPDQNPEVKPRLPAHVVVHENQYQELNLDDIQSYDQTMQAY YASSTSNQKLSSWSQEVTGKLAGESRPHILPYLNSKGLAKR

\section{$\underline{\text { NfrA_Bs }}$}

MNNTIETILNHRSIRSFTDQLLTAEEIDILVKSAQAASTSSYVQAYSIIGVSDPEKKRELSVLAGNQPY VENNGHFFVFCADLHRHQKLAEEKGENISELLENTEMFMVSLIDAALAAQNMSVAAESMGLGICY IGGIRNELDKVTEVLQTPDHVLPLFGLAVGHPANLSGKKPRLPKQAVYHENTYNVNADDFRDTMN AYDQTISDYYRERTNGQREETWSDQILNFMKQKPRTYLNDYVKEKGFNKN

$N f s A_{-} B c$

MNTIIETILNHRSIRHYEDRPLSDEQIRLIVESAQAAATSHFVQAYTILGIQDPGRKQRLAELTGNRH VGTCGHLLIFCADLHKHALAAEMEGVDAQDTLETTEKFMVALIDTALAAQNAALAAESMGLGIC YVGGLRNRLPEVAELLKIPQYVLPLFAMTIGYPADPSAKKPRMAAEHVYFEDEYPADERLLRDLK EYNETVSQYYTKRTDGKRNDTWTGQMAQFFKEPSRVFMKEFVEHQGFDKK 


\section{NfsA Bt}

MNEMIHKMEQHVSVRKYKEESIPKDVVEKMVHAAQHAASSHFVQAYSVIYVTDQELKAKLAELS GNRHVKDCAAFFVCCADLKRLEIACEKHSTEIKHEGVEDFIVATVDASLFAQNLALAAESLGYGIC YIGGIRNNPREVSELLHLPDKVYPVFGMTVGVPDEEHGVKPRLPVAAVLHENGYDEQKYDELLNE YDETMNAYYKERPSNKKNVTWTESMSSFMSKEKRMHMKEFLSERGLNKK

\section{NfsA_Ls}

MSDLIAQMQHHVSVRNFEATPLSAEVKQQLIAAAQSGSSSNFVQAFSIIEVTDLALRTEIATISNSAS YVNQTGTFYVFVADLYRQASMLKAQGQSLAGIQNMEALLVASVDTTIAAEDMAVAAESLGLGIC YIGGIRNDIARVAELLGLPEYTVPLFGLTVGIPKTKNQVKPRLPQINQVAQNQYPRAQFADLKQYD QQIADYYANRGSNQQQADWTSKNLDFFSAPRRPEVGAFLKKQGFTLA

\section{$\underline{\text { Ycnd_Bs }}$}

MNEVIKSLTDHRSIRSYTDEPVAKEQLDQIIQAVQSAPTSINGQQVTVITVQDKERKKKISELSGGQP WIDQAPVFLLFCADFNRAKIALEDLNDIKMEITNGLESVLVGAVDAGIALGTATAAAESLGLGTVPI GAVRGNPQELIELLELPKYVFPVSGLVIGHPADRSAKKPRLPQEAVNYQETYLNQDELTSYIQAYD EKMSEYMNKRTNGKETRNWSQGIASYYERLYYPHIREMLEKQGFKVEK

\section{$\underline{\text { NfsB_Ck }}$}

MDIVSVALKRYSTKAFDPSKQLTADEAEKLKTLLQYSPSSTNSQPWHFIVASTEEGKARVAKSAAG NFVFNERKMLDASHVVVFCAKTAMDDAWLDRVVDQEDADGRFATPEAKAANNKGRRFFADLH RRDLKDDDQWMAKQVYLNVGNFLLGVAAMGLDAVPIEGFDAAVLDAEFGLKEKGYTSLVVVPV GHHSVEDFNAALPKSRLPQETTLTEV

\section{$\underline{\text { NfsB_Kp }}$}

MDIVSVALKRYSTKAFDATKKLTAGEAEQLKTLLQYSPSSTNSQPWHFIVASTDEGKARVAKAAS GTYVFNERKILDASHVVVFCAKTAMDDAWLQRVVDQEEADGRFATPDAKAANHKGRTFFADMH RKELKDDDQWMAKQVYLNVGNFLLGVAAMGLDAVPIEGVDFAILDEEFDLKAQGYTSLVVVPV GHHSVEDFNATLPKSRLPQSTTITEI

\section{NfsV Vv}

MTIVQAAQSRYSTKAFDASRKLPEEKVAAVKELIRMSASSVNSQPWHFIVASSEEGKARIAKATQG GFAFNERKILDASHVVVFCAKTSIDEAYLLDLLQSEDKDGRFADVEAKNGMHAGRSFFVNMHRFD LKDAHHWMEKQVYLNVGTLLLGASAMEIDAVPIEGFDAKVLDEEFGLREKGFTSVVIVPLGYHSE DDFNAKLPKSRWPAETVFTEI

\section{NfsB_Es}

MNLNEIIRTRHTSKAYDNSRKLTAEQQQELLDLLRFSPSSVNSQPWHFFAVTTEEGKAQILPALMD ANQVKAKNAAMTVVFTIKEELNEAHLLQLLEKEQQDGRYDGEEARAANDKGRRFFVGLNSETPE QQREWMTRQAYLALGFLLLGAAAMGLDATPIEGFHPEKMDEVLGLKEKGLRSVVVATIGYRSDA DFNATLPKSRLDQDVVITQL

\section{$\underline{\text { NfsB Vh }}$}

MSHQIITDLNNRYTAKKYDEEKRISQEDMAIIKEAIRLSASSINSQPWKFIVIESDEAKQRFHDTFAN MHQFNQPHAKAASHTILLAYDPKFTKEKFAKRVDAEVTSGHLPADMYDMFMGAYAFAEANTDE NGYNGGWTKAQVYIALGNLLHTLARLGIDSTPMEGVDPTLIGEEFKAELDGHVCEVALAIGYHKD GEDYNHGLPKARLNMDDVITTL

\section{$\underline{\text { YfkO_Bs }}$}

MADLKTQILDAYNFRHATKEFDPNKKVSDSDFEFILETGRLSPSSLGLEPWKFVVVQNPEFREKLR EYTWGAQKQLPTASHFVLILARTAKDIKYDADYIKRHLKEVKQMPQDVYEGYLSKTEEFQKNDL HLLESDRTLFDWASKQTYIALGNMMTAAAQIGVDSCPVEGFQYDHIHRILEEEGLLENGSFDISVM VAFGYRVRDPRPKTRSAVEDVVKWV 


\section{$\underline{\text { YdgI BS }}$}

MIKTNDFMEIMKGRRSIRNYDPTVKISKEEMTEILEEATTAPSSVNAQPWRFLVIDSPEGKEKLAPL ASFNQTQVTTSSAVIAVFADMNNGDYLEEIYSKAVELGYMPQEVKDRQIAALTAHFEKLPAQVNR ETILIDGGLVSMQLMLTARAHGYDTNPIGGYDKENIAETFGLDKERYVPVMLLSIGKAADEGYASY RLPIDKIAEWK

\section{$\underline{\operatorname{AzoR} \text { Vv }}$}

MSRLLVLKSSILGDYSQSNKLVDEFINKFDQQDVVVRDLAQQPLPVLDFQVATALRASGDLSEAQ QAIVDLSDQLIAEIKHADTLIIAAPMYNFTVPTQLKNWIDLIARAGVTFTYTEQGPKGLIEGKKAVV VTTRGGIHKDAASDIITPYLKTVLGFVGITEVEFVYAEALNMGEDFASKGLASASEHLAALTA

\section{$\underline{\text { NemA_St }}$}

MSSAKLFTPLKVGAITATSRVFMAPLTRLRSIEPGDIPTPLMAEYYRQRASAGLIISEATQISAQAKG YAGAPGLHSDEQIAAWKKITQGVHAQGGHMAVQLWHTGRISHASLQPGGQAPVAPSAINAGTRT SLRDENGQAIRVETSTPRALDTHEIPGIVNDFRQAIANAREAGFDLVELHSAHGYLLHQFLSPSSNH RTDRYGGSVENRARLVLEVVDAGIKEWGADRIGIRLSPVGTFQNVDNGPNEEADALYLIEALGKR GIAYLHMSEPDWAGGEPYSDAFREKVRARFHGPIIGAGAYTPEKAEDLIEKGLIDAVAFGRDYIAN PDLVARLQRKAELNPQRPESFYGGGAEGYTDYPTL

\section{NemA_Kp}

MSEAKLFSPLKVGAVTVPNRVFMAPLTRLRSIEPGDIPTPLMGEYYRQRASSGLIITEATQISAQAK GYAGAPGLHSPEQIAAWQKITAGVHAENGHIAVQLWHTGRISHSSLQPGGAAPVAPSALSAGTRTS LRYENGHAIRVDTSMPRALETAEIPGIVNDFRQAVGNARDAGFDLVELHSAHGYLLHQFLSPSANQ RTDQYGGSVENRARLVLEVVDAVSQEWSAERIGIRVSPIGSFQNVDNGPNEEEDALYLISELAKRGI AYLHMSEPDWAGGKPYSEAFRQKVRDRFPGVIIGAGAYTVEKANDLINKGLIDAVAFGRDYIANP DLVARLQKKAALNPQRPESFYGGGAEGYTDYPTL

\section{$\underline{\text { NemA_Vv }}$}

MSKLFEPTQLKQLDLLNRVVMAPMTRARTSQPGNIPNQMMATYYKQRATAGLIISEATQISDDSQ GYSFTPGVYTDEQVAGWKLVTQAVKSQGAAMFCQLWHVGRVSHPVFQKGEQPIAPSALAPVETK VWIADEQGNGNMVDCVEPRAMTQADIDRVVSDFAYAAKRAIEAGFDGIEIHGGNGYLIDQFLRTN SNHRTDNYGGTRENRLRFLLEVVDAVSKAIGANKVGVRLAPFITFKDMNCPDIVPTILDASKQLQA RDIAYLHLSEADWDDAPVIPESFRIELREYFTNTIIVAGSYTQARADEVLEKGYADLVAFGRPFVSN PDLVARLKHQQPLAELDGATLFGGNERGYTDYTALHV

\section{$\underline{\text { YwrO_Bs }}$}

MKILVLAVHPHMETSVVNKAWTEELSQHDNITVRELYKEYPNEAIDVAKEQQLCEQYDRIVFQFP FYWYGSPPLLKKWQDLVLTYGWAFGSEGNALHGKELMLAVSTGSEAEKYQAGGANHYSISELLK PFQATSNLIGMKYLPPYVFYGVNDATAEDISLSAKRLAEYIQQPFV

\section{$\underline{\text { YwrOLLi }}$}

MKTLVIIAHPNIENSRVNRVWKEALLKSTEEVAIHELYEVYPNWDIDVTFEQQQLQNYDKVIIQFPF YWYSYPPLLKKWFDDVFSYGWAYGSKGDKMAGKKLALAVSIGDKKYNYKEDAPIGYSLDTLLTP FRATINHIRADYRGAHTIYGSSFEVTDEEIVENAYVYANKFIKTLN 


\section{Appendix 2:}

\begin{tabular}{|c|c|c|c|c|c|c|c|}
\hline $\begin{array}{c}\text { Mutation R12X } \\
\text { Fold improvement (EF5/F-miso/Both) } \\
\text { Possible redundancy codon }\end{array}$ & $\begin{array}{c}\mathrm{S} \\
(1.6 / 1.7 / 3.3) \\
\mathrm{MGC}\end{array}$ & & & & & & \\
\hline S40X & $\begin{array}{c}\mathrm{T} \\
(1.6 / 1.7 / 3.3) \\
\text { WCT }\end{array}$ & $\begin{array}{c}\text { W } \\
(1.4 / 1.8 / 3.2) \\
\text { TSG }\end{array}$ & & & & & \\
\hline S41X & $\begin{array}{c}\mathbf{M} \\
(2.1 / 2.9 / 5.0)\end{array}$ & $\begin{array}{c}\mathbf{Q} \\
(2.1 / 2.6 / 4.7)\end{array}$ & $\begin{array}{c}\mathrm{Y} \\
(1.9 / 2.6 / 4.5)\end{array}$ & $\begin{array}{c}\text { C } \\
(1.9 / 2.6 / 4.5)\end{array}$ & $\begin{array}{c}\mathbf{G} \\
(1.8 / 1.6 / 3.4)\end{array}$ & $\mathrm{C}+\mathrm{Y}$ & $\mathrm{G}+\mathrm{C}$ \\
\hline F42 & $\begin{array}{c}\text { none } \\
\mathbf{N} \\
(2.2 / 2.0 / 4.2) \\
\text { none }\end{array}$ & $\begin{array}{c}\text { none } \\
\text { I } \\
(1.9 / 2.2 / 4.1) \\
\text { WTC }\end{array}$ & $\begin{array}{c}\text { TMT } \\
\mathbf{W} \\
(1.8 / 2.0 / 3.8) \\
\text { none }\end{array}$ & $\begin{array}{c}\text { WGT } \\
\mathbf{A} \\
(2.0 / 1.6 / 3.6) \\
\text { none }\end{array}$ & $\begin{array}{c}\text { RGT } \\
\mathbf{R} \\
(1.5 / 2.0 / 3.5) \\
\text { none }\end{array}$ & $\begin{array}{c}\text { TVT } \\
\mathbf{H} \\
(1.8 / 1.6 / 3.4) \\
\text { none }\end{array}$ & DGT \\
\hline L43 & $\begin{array}{c}\mathbf{D} \\
(1.4 / 1.3 / 2.7) \\
\text { none }\end{array}$ & $\begin{array}{c}\mathbf{E} \\
(1.3 / 1.1 / 2.4) \\
\text { none }\end{array}$ & & & & & \\
\hline C45 & $\begin{array}{c}\mathbf{M} \\
(2.2 / 2.0 / 4.2) \\
\text { none }\end{array}$ & $\begin{array}{c}\mathbf{E} \\
(2.2 / 1.7 / 3.9) \\
\text { none }\end{array}$ & $\begin{array}{c}\text { L } \\
(2.0 / 1.7 / 3.7) \\
\text { none }\end{array}$ & $\begin{array}{c}\mathbf{H} \\
(2.2 / 1.1 / 3.3) \\
\text { none }\end{array}$ & $\begin{array}{c}\text { D } \\
(1.7 / 1.1 / 2.8) \\
\text { none }\end{array}$ & & \\
\hline F83 & $\begin{array}{c}\text { D } \\
(1.7 / 2.1 / 3.8) \\
\text { none }\end{array}$ & $\begin{array}{c}\mathbf{G} \\
(1.6 / 1.8 / 3.4) \\
\text { none }\end{array}$ & $\begin{array}{c}\text { A } \\
(1.7 / 1.3 / 3.0) \\
\text { none }\end{array}$ & $\begin{array}{c}\mathbf{R} \\
(1.7 / 1.4 / 3.1) \\
\text { none }\end{array}$ & & & \\
\hline L103 & $\begin{array}{c}\mathrm{V} \\
(1.7 / 1.3 / 3.0) \\
\text { STW } \\
\end{array}$ & $\begin{array}{c}\mathrm{Q} \\
(1.6 / 1.1 / 2.7) \\
\text { CWG }\end{array}$ & & & & & \\
\hline N134 & $\begin{array}{c}\text { A } \\
(1.7 / 2.3 / 4.0) \\
\text { none }\end{array}$ & $\begin{array}{c}\mathbf{R} \\
(1.8 / 1.9 / 3.7) \\
\text { none }\end{array}$ & $\begin{array}{c}\mathbf{W} \\
(1.6 / 0.8 / 2.4) \\
\text { none }\end{array}$ & & & & \\
\hline H215 & $\begin{array}{c}\mathbf{R} \\
(1.4 / 1.5 / 2.9) \\
\text { CRT }\end{array}$ & $\begin{array}{c}\mathbf{K} \\
(1.3 / 1.6 / 2.9) \\
\text { none }\end{array}$ & & & & & \\
\hline
\end{tabular}

Table A2-1 All possible degenerate codons which designated only the NfsA_Ec wild type amino acid residue or the indicated mutant residue. Mutations chosen for inclusion into a multi-mutant gene library are marked as red while other possible codons are marked as blue. Codons calculated using online tool (http://guinevere.otago.ac.nz/cgi-bin/aef/AA-Calculator.pl). 


\section{Appendix 3:}

\begin{tabular}{ccccccccccc}
\hline Random Clone & R12S & S40T & S41C & S41Y & F42I & C45L & C45M & L103V & N134A & H215R \\
\hline 1 & & & $\checkmark$ & & $\checkmark$ & & & & $\checkmark$ & $\checkmark$ \\
2 & $\checkmark$ & $\checkmark$ & & $\checkmark$ & & & & & $\checkmark$ & \\
3 & $\checkmark$ & & & & & $\checkmark$ & & $\checkmark$ & & \\
4 & & & $\checkmark$ & & $\checkmark$ & & & $\checkmark$ & & \\
5 & $\checkmark$ & $\checkmark$ & & & & & & & & $\checkmark$ \\
6 & & & & $\checkmark$ & $\checkmark$ & & $\checkmark$ & $\checkmark$ & & \\
7 & & & & & & $\checkmark$ & & $\checkmark$ & $\checkmark$ & \\
8 & & $\checkmark$ & $\checkmark$ & & $\checkmark$ & & & $\checkmark$ & $\checkmark$ & $\checkmark$ \\
9 & & $\checkmark$ & & & & & & $\checkmark$ & & \\
10 & $\checkmark$ & & & $\checkmark$ & $\checkmark$ & & & & & $\checkmark$ \\
\hline
\end{tabular}

Table A3-1Table of mutations found in random clones from NfsA_Ec mutant library designed to specify either the wild type amino acid residue or the designated mutation at each targeted site.

\begin{tabular}{ccccc}
\hline Random Clone & $\mathbf{4 1}$ & $\mathbf{4 5}$ & $\mathbf{8 3}$ & $\mathbf{1 3 4}$ \\
\hline 1 & $\mathrm{~K}$ & $\mathrm{H}$ & $\mathrm{V}$ & $\mathrm{STOP}$ \\
2 & $\mathrm{P}$ & $\mathrm{A}$ & $\mathrm{S}$ & $\mathrm{S}$ \\
3 & $\mathrm{E}$ & $\mathrm{Q}$ & $\mathrm{C}$ & $\mathrm{G}$ \\
4 & $\mathrm{STOP}$ & $\mathrm{D}$ & $\mathrm{L}$ & $\mathrm{R}$ \\
5 & $\mathrm{~V}$ & $\mathrm{~S}$ & $\mathrm{P}$ & $\mathrm{C}$ \\
\hline
\end{tabular}

Table A3-2 Table of mutations found in random clones from NfsA_Ec mutant library designed to be able to have any of the 20 common amino acid residues, or one stop codon, at sites 41, 45, 83 and 134. 


\section{References}

Abate-Daga, D., Andreu, N., Camacho-Sánchez, J., Alemany, R., Herance, R., Millán, O., and Fillat, C. (2011). Oncolytic adenoviruses armed with thymidine kinase can be traced by PET imaging and show potent antitumoural effects by ganciclovir dosing. PLoS ONE $6, \mathrm{e} 26142$.

Ackerley, D.F., Gonzalez, C.F., Park, C.H., Blake, R., Keyhan, M., and Matin, A. (2004a). Chromate-Reducing Properties of Soluble Flavoproteins from Pseudomonas putida and Escherichia coli. Appl. Environ. Microbiol. 70, 873-882.

Adair, R.A., Roulstone, V., Scott, K.J., Morgan, R., Nuovo, G.J., Fuller, M., Beirne, D., West, E.J., Jennings, V.A., Rose, A., et al. (2012). Cell carriage, delivery, and selective replication of an oncolytic virus in tumor in patients. Sci. Transl. Med. 4, 138 ra77.

Adams, M.A., and Jia, Z. (2006). Modulator of drug activity B from Escherichia coli: crystal structure of a prokaryotic homologue of DT-diaphorase. J. Mol. Biol. 359, 455465.

al-Ramadi, B.K., Fernandez-Cabezudo, M.J., El-Hasasna, H., Al-Salam, S., Bashir, G., and Chouaib, S. (2009). Potent anti-tumor activity of systemically-administered IL2expressing Salmonella correlates with decreased angiogenesis and enhanced tumor apoptosis. Clin. Immunol. 130, 89-97.

Altaner, C. (2008). Prodrug cancer gene therapy. Cancer Lett. 270, 191-201.

Anlezark, G.M., Melton, R.G., Sherwood, R.F., Coles, B., Friedlos, F., and Knox, R.J. (1992). The bioactivation of 5-(aziridin-1-yl)-2,4-dinitrobenzamide (CB1954)-I: Purification and properties of a nitroreductase enzyme from Escherichia coli-A potential enzyme for antibody-directed enzyme prodrug therapy (ADEPT). Biochem. Pharmacol. 44, 2289-2295.

Anlezark, G.M., Melton, R.G., Sherwood, R.F., Wilson, W.R., Denny, W.A., Palmer, B.D., Knox, R.J., Friedlos, F., and Williams, A. (1995). Bioactivation of dinitrobenzamide mustards by an E. coli B nitroreductase. Biochem. Pharmacol. 50, 609618.

Anlezark, G.M., Vaughan, T., Fashola-Stone, E., Michael, N.P., Murdoch, H., Sims, M.A., Stubbs, S., Wigley, S., and Minton, N.P. (2002). Bacillus amyloliquefaciens orthologue of Bacillus subtilis ywrO encodes a nitroreductase enzyme which activates the prodrug CB 1954. Microbiology 148, 297-306.

Asosingh, K., De Raeve, H., De Ridder, M., Storme, G.A., Willems, A., Van Riet, I., Van Camp, B., and Vanderkerken, K. (2005). Role of the hypoxic bone marrow microenvironment in 5T2MM murine myeloma tumor progression. Haematologica 90, 810-817.

Atwell, G.J., Yang, S., Pruijn, F.B., Pullen, S.M., Hogg, A., Patterson, A.V., Wilson, W.R., and Denny, W.A. (2007). Synthesis and structure-activity relationships for 2,4dinitrobenzamide-5-mustards as prodrugs for the Escherichia coli nfsB nitroreductase in gene therapy. J. Med. Chem. 50, 1197-1212. 
Barak, Y., Thorne, S.H., Ackerley, D.F., Lynch, S.V., Contag, C.H., and Matin, A. (2006). New enzyme for reductive cancer chemotherapy, YieF, and its improvement by directed evolution. Mol. Cancer Ther. 5, 97-103.

Barbé, S., Van Mellaert, L., Theys, J., Geukens, N., Lammertyn, E., Lambin, P., and Anné, J. (2005). Secretory production of biologically active rat interleukin-2 by Clostridium acetobutylicum DSM792 as a tool for anti-tumor treatment. FEMS Microbiol. Lett. 246, 67-73.

Baril, P., Martin-Duque, P., and Vassaux, G. (2010). Visualization of gene expression in the live subject using the $\mathrm{Na} / \mathrm{I}$ symporter as a reporter gene: applications in biotherapy. Br. J. Pharmacol. 159, 761-771.

Barton, K.N., Tyson, D., Stricker, H., Lew, Y.S., Heisey, G., Koul, S., De la Zerda, A., Yin, F.-F., Yan, H., Nagaraja, T.N., et al. (2003). GENIS: Gene Expression of Sodium Iodide Symporter for Noninvasive Imaging of Gene Therapy Vectors and Quantification of Gene Expression in vivo. Mol. Ther. 8, 508-518.

Barton, K.N., Stricker, H., Brown, S.L., Elshaikh, M., Aref, I., Lu, M., Pegg, J., Zhang, Y., Karvelis, K.C., Siddiqui, F., et al. (2008). Phase I study of noninvasive imaging of adenovirus-mediated gene expression in the human prostate. Mol. Ther. 16, 1761-1769.

Barton, K.N., Stricker, H., Elshaikh, M.A., Pegg, J., Cheng, J., Zhang, Y., Karvelis, K.C., Lu, M., Movsas, B., and Freytag, S.O. (2011). Feasibility of Adenovirus-Mediated hNIS Gene Transfer and ${ }^{131}$ I Radioiodine Therapy as a Definitive Treatment for Localized Prostate Cancer. Mol. Ther. 19, 1353-1359.

Bennett, B.D., Kimball, E.H., Gao, M., Osterhout, R., Van Dien, S.J., and Rabinowitz, J.D. (2009). Absolute Metabolite Concentrations and Implied Enzyme Active Site Occupancy in Escherichia coli. Nat. Chem. Biol. 5, 593-599.

Bettegowda, C., Dang, L.H., Abrams, R., Huso, D.L., Dillehay, L., Cheong, I., Agrawal, N., Borzillary, S., McCaffery, J.M., Watson, E.L., et al. (2003). Overcoming the hypoxic barrier to radiation therapy with anaerobic bacteria. PNAS 100, 15083-15088.

Bettegowda, C., Foss, C.A., Cheong, I., Wang, Y., Diaz, L., Agrawal, N., Fox, J., Dick, J., Dang, L.H., Zhou, S., et al. (2005). Imaging bacterial infections with radiolabeled 1(2'-deoxy-2'- fluoro- $\beta$-D-arabinofuranosyl)-5-iodouracil. PNAS 102, 1145-1150.

Beyer, T., Townsend, D.W., Brun, T., Kinahan, P.E., Charron, M., Roddy, R., Jerin, J., Young, J., Byars, L., and Nutt, R. (2000). A Combined PET/CT Scanner for Clinical Oncology. J. Nucl. Med. 41, 1369-1379.

Bhaumik, S., and Gambhir, S.S. (2002). Optical imaging of Renilla luciferase reporter gene expression in living mice. PNAS 99, 377-382.

Bhaumik, S., Sekar, T.V., Depuy, J., Klimash, J., and Paulmurugan, R. (2011). Noninvasive optical imaging of nitroreductase gene-directed enzyme prodrug therapy system in living animals. Gene Ther. 19, 295-302. 
Black, M.E., Newcomb, T.G., Wilson, H.M., and Loeb, L.A. (1996). Creation of drugspecific herpes simplex virus type 1 thymidine kinase mutants for gene therapy. PNAS. $93,3525-3529$.

Bleehen, N.M., Maughan, T.S., Workman, P., Newman, H.F., Stenning, S., and Ward, R. (1991). The combination of multiple doses of etanidazole and pimonidazole in 48 patients: a toxicity and pharmacokinetic study. Radiother. Oncol. 20 Suppl 1, 137-142.

Boland, M.P., Knox, R.J., and Roberts, J.J. The differences in kinetics of rat and human DT diaphorase result in a differential sensitivity of derived cell lines to CB 1954 (5(aziridin-1-yl)-2,4-dinitrobenzamide). Biochem. Pharmacol. 41, 867-875.

Bornscheuer, U., and Kazlauskas, R.J. (2011). Survey of protein engineering strategies. Curr. Protoc. Protein. Sci. 66: 26.7.1-26.7.14.

Brader, P., Stritzker, J., Riedl, C.C., Zanzonico, P., Cai, S., Burnazi, E.M., Ghani, E.R., Hricak, H., Szalay, A.A., Fong, Y., et al. (2008). Escherichia coli Nissle 1917 Facilitates Tumor Detection by Positron Emission Tomography and Optical Imaging. Clin. Cancer Res. 14, $2295-2302$.

Brader, P., Kelly, K.J., Chen, N., Yu, Y.A., Zhang, Q., Zanzonico, P., Burnazi, E.M., Ghani, R.E., Serganova, I., Hricak, H., et al. (2009a). Imaging a Genetically Engineered Oncolytic Vaccinia Virus (GLV-1h99) Using a Human Norepinephrine Transporter Reporter Gene. Clin. Cancer Res. 15, 3791-3801.

Brader, P., Kelly, K., Gang, S., Shah, J.P., Wong, R.J., Hricak, H., Blasberg, R.G., Fong, Y., and Gil, Z. (2009b). Imaging of Lymph Node Micrometastases Using an Oncolytic Herpes Virus and $\left[{ }^{18}\right.$ F]FEAU PET. PLoS ONE 4, e4789.

Breitbach, C.J., Reid, T., Burke, J., Bell, J.C., and Kirn, D.H. (2010). Navigating the clinical development landscape for oncolytic viruses and other cancer therapeutics: No shortcuts on the road to approval. Cytokine Growth Factor Rev. 21, 85-89.

Breitbach, C.J., Burke, J., Jonker, D., Stephenson, J., Haas, A.R., Chow, L.Q.M., Nieva, J., Hwang, T.-H., Moon, A., Patt, R., et al. (2011a). Intravenous delivery of a multimechanistic cancer-targeted oncolytic poxvirus in humans. Nature 477, 99-102.

Breitbach, C.J., De Silva, N.S., Falls, T.J., Aladl, U., Evgin, L., Paterson, J., Sun, Y.Y., Roy, D.G., Rintoul, J.L., Daneshmand, M., et al. (2011b). Targeting tumor vasculature with an oncolytic virus. Mol. Ther. 19, 886-894.

Bremner, J.C. (1993). Assessing the bioreductive effectiveness of the nitroimidazole RSU1069 and its prodrug RB6145: with particular reference to in vivo methods of evaluation. Cancer Metastasis Rev. 12, 177-193.

Brezden, C.B., McClelland, R.A., Rauth, A. M., (1997). Apoptosis and 1-methyl-2nitroimidazole toxicity in CHO cells. Br. J. Cancer. 76, 180-188.

Briat, A., and Vassaux, G. (2006). Preclinical applications of imaging for cancer gene therapy. Expert Rev. Mol. Med. 8, 1-19. 
Bridgewater, J.A., Knox, R.J., Pitts, J.D., Collins, M.K., and Springer, C.J. (1997). The bystander effect of the nitroreductase/CB1954 enzyme/prodrug system is due to a cellpermeable metabolite. Hum. Gene Ther. 8, 709-717.

Bryant, C., and DeLuca, M. (1991). Purification and characterization of an oxygeninsensitive $\mathrm{NAD}(\mathrm{P}) \mathrm{H}$ nitroreductase from Enterobacter cloacae. J. Biol. Chem. 266, 4119-4125.

Bryant, D.W., McCalla, D.R., Leeksma, M., and Laneuville, P. (1981). Type I nitroreductases of Escherichia coli. Can. J. Microbiol. 27, 81-86.

Buchanan, A., Ferraro, F., Rust, S., Sridharan, S., Franks, R., Dean, G., McCourt, M., Jermutus, L., and Minter, R. (2012). Improved drug-like properties of therapeutic proteins by directed evolution. Protein Eng. Des. Sel. 25, 631-638.

Burg, B.V. den, Vriend, G., Veltman, O.R., Venema, G., and Eijsink, V.G.H. (1998). Engineering an enzyme to resist boiling. PNAS 95, 2056-2060.

Busch, T.M., Hahn, S.M., Evans, S.M., and Koch, C.J. (2000). Depletion of Tumor Oxygenation during Photodynamic Therapy: Detection by the Hypoxia Marker EF3 [2(2-Nitroimidazol-1[H]-yl)-N-(3,3,3-trifluoropropyl)acetamide]. Cancer Res. 60, 26362642.

Carey, J., Brynda, J., Wolfová, J., Grandori, R., Gustavsson, T., Ettrich, R., and Smatanová, I.K. (2007). WrbA bridges bacterial flavodoxins and eukaryotic NAD(P)H:quinone oxidoreductases. Protein Science 16, 2301-2305.

Carey, R.W., Holland, J.F., Whang, H.Y., Neter, E., and Bryant, B. (1967). Clostridial oncolysis in man. Eur. J. Cancer (1965) 3, 37-46.

Carlin, S., and Humm, J.L. (2012). PET of Hypoxia: Current and Future Perspectives. J. Nucl. Med. 53, 1171-1174.

Chatterjee, P.K., and Sternberg, N.L. (1995). A general genetic approach in Escherichia coli for determining the mechanism(s) of action of tumoricidal agents: application to DMP 840, a tumoricidal agent. PNAS 92, 8950.

Chen, R., Parry, J.J., Akers, W.J., Berezin, M.Y., Naqa, I.M.E., Achilefu, S., Edwards, W.B., and Rogers, B.E. (2010). Multimodality Imaging of Gene Transfer with a Receptor-Based Reporter Gene. J. Nucl. Med. 51, 1456-1463.

Chiocca, E.A. (2002). Oncolytic viruses. Nat. Rev. Cancer 2, 938-950.

Chitneni, S.K., Bida, G.T., Dewhirst, M.W., and Zalutsky, M.R. (2012). A simplified synthesis of the hypoxia imaging agent 2-(2-Nitro-1H-imidazol-1-yl)-N-(2,2,3,3,3[(18)F]pentafluoropropyl)-acetamide ([(18)F]EF5). Nucl. Med. Biol. 39, 1012-1018.

Choi, J.-W., Lee, J., Nishi, K., Kim, Y.-S., Jung, C.-H., and Kim, J.-S. (2008). Crystal structure of a minimal nitroreductase, ydjA, from Escherichia coli $\mathrm{K} 12$ with and without FMN cofactor. J. Mol. Biol. 377, 258-267. 
Chung-Faye, G., Palmer, D., Anderson, D., Clark, J., Downes, M., Baddeley, J., Hussain, S., Murray, P.I., Searle, P., Seymour, L., et al. (2001). Virus-directed, enzyme prodrug therapy with nitroimidazole reductase: a phase I and pharmacokinetic study of its prodrug, CB1954. Clin. Cancer Res. 7, 2662-2668.

Cobb, L.M., Connors, T.A., Elson, L.A., Khan, A.H., Mitchley, B.C.V., Ross, W.C.J., and Whisson, M.E. (1969). 2,4-Dinitro-5-ethyleneiminobenzamide (CB 1954): A potent and selective inhibitor of the growth of the Walker carcinoma 256. Biochem. Pharmacol. $18,1519-1527$.

Coenen, H.H., Elsinga, P.H., Iwata, R., Kilbourn, M.R., Pillai, M.R.A., Rajan, M.G.R., Wagner Jr., H.N., and Zaknun, J.J. (2010). Fluorine-18 radiopharmaceuticals beyond [18F]FDG for use in oncology and neurosciences. Nucl. Med. Bio. 37, 727-740.

Cortial, S., Chaignon, P., Iorga, B.I., Aymerich, S., Truan, G., Gueguen-Chaignon, V., Meyer, P., Moréra, S., and Ouazzani, J. (2010). NADH oxidase activity of Bacillus subtilis nitroreductase NfrA1: insight into its biological role. FEBS Lett. 584, 3916-3922.

Cronin, M., Akin, A.R., Collins, S.A., Meganck, J., Kim, J.-B., Baban, C.K., Joyce, S.A., Van Dam, G.M., Zhang, N., Van Sinderen, D., et al. (2012). High Resolution In Vivo Bioluminescent Imaging for the Study of Bacterial Tumour Targeting. PLoS ONE 7, e30940.

Dachs, G.., and Tozer, G.. (2000). Hypoxia modulated gene expression: angiogenesis, metastasis and therapeutic exploitation. Eur. J. Cancer 36, 1649-1660.

Dang, L.H., Bettegowda, C., Huso, D.L., Kinzler, K.W., and Vogelstein, B. (2001). Combination bacteriolytic therapy for the treatment of experimental tumors. PNAS 98, 15155-15160.

Das, T., Banerjee, S., Samuel, G., Sarma, H.D., Korde, A., Venkatesh, M., and Pillai, M.R.A. (2003). 99mTc-labeling studies of a modified metronidazole and its biodistribution in tumor bearing animal models. Nucl. Med. Biol. 30, 127-134.

Datsenko, K.A., and Wanner, B.L. (2000). One-step inactivation of chromosomal genes in Escherichia coli K-12 using PCR products. PNAS 97, 6640-6645.

Deller, S., Macheroux, P., and Sollner, S. (2008). Flavin-dependent quinone reductases. Cell. Mol. Life Sci. 65, 141-160.

Dempsey, M.F., Wyper, D., Owens, J., Pimlott, S., Papanastassiou, V., Patterson, J., Hadley, D.M., Nicol, A., Rampling, R., and Brown, S.M. (2006). Assessment of ${ }^{123}$ IFIAU imaging of herpes simplex viral gene expression in the treatment of glioma. Nucl. Med. Commun. 27, 611-617.

Denny, W.A. (2000). The role of hypoxia-activated prodrugs in cancer therapy. Lancet Oncol. 1, 25-29.

Denny, W.A., and Wilson, W.R. (1993). Bioreducible mustards: a paradigm for hypoxiaselective prodrugs of diffusible cytotoxins (HPDCs). Cancer Metastasis Rev. 12, 135151. 
De Visser, K.E., Eichten, A., and Coussens, L.M. (2006). Paradoxical roles of the immune system during cancer development. Nat. Rev. Cancer 6, 24-37.

DeVita, V.T., and Chu, E. (2008). A History of Cancer Chemotherapy. Cancer Res. 68, 8643-8653.

Dokić, D.D. (2005). [Technetium-99m radiopharmaceuticals for in vivo diagnostics]. Med. Pregl. 58, 180-184.

Doss, M., Zhang, J.J., Bélanger, M.-J., Stubbs, J.B., Hostetler, E.D., Alpaugh, K., Kolb, H.C., and Yu, J.Q. (2010). Biodistribution and radiation dosimetry of the hypoxia marker $18 \mathrm{~F}-\mathrm{HX} 4$ in monkeys and humans determined by using whole-body PET/CT. Nucl. Med. 31, 1016-1024.

Duarte, S., Carle, G., Faneca, H., Lima, M.C.P. de, and Pierrefite-Carle, V. (2012). Suicide gene therapy in cancer: Where do we stand now? Cancer Lett. 324, 160-170.

Eisenthal, R., Danson, M.J., and Hough, D.W. (2007). Catalytic efficiency and $k_{c a t} / K_{M \text { : a }}$ useful comparator? Trends Biotech. 25, 247-249.

Emptage, C.D., Knox, R.J., Danson, M.J., and Hough, D.W. (2009). Nitroreductase from Bacillus licheniformis: A stable enzyme for prodrug activation. Biochem. Pharmacol. 77, 21-29.

Fernández, S., Dematteis, S., Giglio, J., Cerecetto, H., and Rey, A. (2012). Synthesis, in vitro and in vivo characterization of two novel ${ }^{68} \mathrm{Ga}$-labelled 5-nitroimidazole derivatives as potential agents for imaging hypoxia. Nucl. Med. Biol. 40, 273-279.

Fontanellas, A., Hervas-Stubbs, S., Sampedro, A., Collantes, M., Azpilicueta, A., Mauleón, I., Pañeda, A., Quincoces, G., Prieto, J., Melero, I., et al. (2009). PET imaging of thymidine kinase gene expression in the liver of non-human primates following systemic delivery of an adenoviral vector. Gene Ther. 16, 136-141.

Forbes, N.S. (2010). Engineering the perfect (bacterial) cancer therapy. Nat. Rev. Cancer $10,785-794$.

Forbes, N.S., Munn, L.L., Fukumura, D., and Jain, R.K. (2003). Sparse initial entrapment of systemically injected Salmonella typhimurium leads to heterogeneous accumulation within tumors. Cancer Res. 63, 5188-5193.

Fralick, J.A. (1996). Evidence that TolC is required for functioning of the Mar/AcrAB efflux pump of Escherichia coli. J.Bacteriol. 178, 5803.

Freytag, S.O., Movsas, B., Aref, I., Stricker, H., Peabody, J., Pegg, J., Zhang, Y., Barton, K.N., Brown, S.L., Lu, M., et al. (2007). Phase I trial of replication-competent adenovirus-mediated suicide gene therapy combined with IMRT for prostate cancer. Mol. Ther. 15, 1016-1023.

Gambhir, S.S., Bauer, E., Black, M.E., Liang, Q., Kokoris, M.S., Barrio, J.R., Iyer, M., Namavari, M., Phelps, M.E., and Herschman, H.R. (2000). A mutant herpes simplex virus type 1 thymidine kinase reporter gene shows improved sensitivity for imaging reporter gene expression with positron emission tomography. PNAS. 97, 2785. 
Ganai, S., Arenas, R.B., and Forbes, N.S. (2009). Tumour-targeted delivery of TRAIL using Salmonella typhimurium enhances breast cancer survival in mice. Br. J. Cancer 101, 1683-1691.

Ganjoo, K.N., Cranmer, L.D., Butrynski, J.E., Rushing, D., Adkins, D., Okuno, S.H., Lorente, G., Kroll, S., Langmuir, V.K., and Chawla, S.P. (2011). A phase I study of the safety and pharmacokinetics of the hypoxia-activated prodrug TH-302 in combination with doxorubicin in patients with advanced soft tissue sarcoma. Oncology 80, 50-56.

Garber, K. (2006). China Approves World's First Oncolytic Virus Therapy For Cancer Treatment. J. Natl. Cancer Inst. 98, 298-300.

Giglio, J., Fernández, S., Pietzsch, H.-J., Dematteis, S., Moreno, M., Pacheco, J.P., Cerecetto, H., and Rey, A. (2012). Synthesis, in vitro and in vivo characterization of novel 99mTc-'4+1'-labeled 5-nitroimidazole derivatives as potential agents for imaging hypoxia. Nucl. Med. Biol. 39, 679-686.

Gilad, A.A., Ziv, K., McMahon, M.T., Zijl, P.C.M. van, Neeman, M., and Bulte, J.W.M. (2008). MRI Reporter Genes. J. Nucl. Med. 49, 1905-1908.

Glieder, A., Meinhold, P. (2003) High-Throughput Screens Based on NAD(P)H Depletion in Methods in Molecular Biology, vol. 230: Directed Enzyme Evolution: Screening and Selection Methods, F.H. Arnold and G. Georgiou. (Humana Press Inc., Totowa, NJ).

Gollamudi, R., Ghalib, M.H., Desai, K.K., Chaudhary, I., Wong, B., Einstein, M., Coffey, M., Gill, G.M., Mettinger, K., Mariadason, J.M., et al. (2009). Intravenous administration of Reolysin ${ }^{\circledR}$, a live replication competent RNA virus is safe in patients with advanced solid tumors. Invest. New Drugs 28, 641-649.

Gonzalez, C., Ackerley, D., Keyhan, M., Matin, A., and Blake, R. (2005a). Evaluation of class II chromate reductases and their bioremediation potential. In Remediation of Contaminated Sediments, P. White, and R. Olfenbuttel, eds. (Columbus, OH: Battelle Press.)

Gonzalez, C.F., Ackerley, D.F., Lynch, S.V., and Matin, A. (2005b). ChrR, a soluble quinone reductase of Pseudomonas putida that defends against $\mathrm{H}_{2} \mathrm{O}_{2}$. J. Biol. Chem. 280, 22590-22595.

Goodwin, A., Kersulyte, D., Sisson, G., Veldhuyzen van Zanten, S.J., Berg, D.E., and Hoffman, P.S. (1998). Metronidazole resistance in Helicobacter pylori is due to null mutations in a gene $(\mathrm{rdxA})$ that encodes an oxygen-insensitive NADPH nitroreductase. Mol. Microbiol. 28, 383-393.

Gray, L.H., Conger, A.D., Ebert, M., Hornsey, S., and Scott, O.C.A. (1953). The Concentration of Oxygen Dissolved in Tissues at the Time of Irradiation as a Factor in Radiotherapy. Br. J. Radiol. 26, 638-648.

Grove, J.I., Lovering, A.L., Guise, C., Race, P.R., Wrighton, C.J., White, S.A., Hyde, E.I., and Searle, P.F. (2003). Generation of Escherichia coli Nitroreductase Mutants Conferring Improved Cell Sensitization to the Prodrug CB1954. Cancer Res. 63, 5532 5537. 
Grunbaum, Z., Freauff, S.J., Krohn, K.A., Wilbur, D.S., Magee, S., and Rasey, J.S. (1987). Synthesis and characterization of congeners of misonidazole for imaging hypoxia. J. Nucl. Med. 28, 68-75.

Guise, C.P., Wang, A.T., Theil, A., Bridewell, D.J., Wilson, W.R., and Patterson, A.V. (2007a). Identification of human reductases that activate the dinitrobenzamide mustard prodrug PR-104A: a role for NADPH:cytochrome P450 oxidoreductase under hypoxia. Biochem. Pharmacol. 74, 810-820.

Guise, C.P., Grove, J.I., Hyde, E.I., and Searle, P.F. (2007b). Direct positive selection for improved nitroreductase variants using SOS triggering of bacteriophage lambda lytic cycle. Gene Ther. 14, 690-698.

Guise, C.P., Abbattista, M.R., Singleton, R.S., Holford, S.D., Connolly, J., Dachs, G.U., Fox, S.B., Pollock, R., Harvey, J., Guilford, P., et al. (2010). The bioreductive prodrug PR-104A is activated under aerobic conditions by human aldo-keto reductase 1C3. Cancer Res. 70, 1573-1584.

Haddad, D., Chen, C.-H., Carlin, S., Silberhumer, G., Chen, N.G., Zhang, Q., Longo, V., Carpenter, S.G., Mittra, A., Carson, J., et al. (2012). Imaging Characteristics, Tissue Distribution, and Spread of a Novel Oncolytic Vaccinia Virus Carrying the Human Sodium Iodide Symporter. PLoS ONE 7, e41647.

Harrington, K.J., Vile, R.G., Melcher, A., Chester, J., and Pandha, H.S. (2010). Clinical trials with oncolytic reovirus: Moving beyond phase I into combinations with standard therapeutics. Cytokine Growth Factor Rev. 21, 91-98.

Helsby, N.A., Ferry, D.M., Patterson, A.V., Pullen, S.M., and Wilson, W.R. (2004). 2Amino metabolites are key mediators of CB 1954 and SN 23862 bystander effects in nitroreductase GDEPT. Br. J. Cancer 90, 1084-1092.

Heo, J., Reid, T., Ruo, L., Breitbach, C.J., Rose, S., Bloomston, M., Cho, M., Lim, H.Y., Chung, H.C., Kim, C.W., et al. (2013). Randomized dose-finding clinical trial of oncolytic immunotherapeutic vaccinia JX-594 in liver cancer. Nat. Med. 19, 329-336.

Heppner, P.D.F., and Möse, J.R. (1978). The liquefaction (oncolysis) of malignant gliomas by a non pathogenic Clostridium. Acta. Neurochir. 42, 123-125.

Herrmann, K., Benz, M.R., Krause, B.J., Pomykala, K.L., Buck, A.K., and Czernin, J. (2011). (18)F-FDG-PET/CT in evaluating response to therapy in solid tumors: where we are and where we can go. J. Nucl. Med. Mol. Imaging 55, 620-632.

Hicks, R.J., and Hofman, M.S. (2012). Is there still a role for SPECT-CT in oncology in the PET-CT era? Nat. Rev. Clin. Oncol.9, 712-720.

Hill, P.J., Stritzker, J., Scadeng, M., Geissinger, U., Haddad, D., Basse-Lüsebrink, T.C., Gbureck, U., Jakob, P., and Szalay, A.A. (2011). Magnetic Resonance Imaging of Tumors Colonized with Bacterial Ferritin-Expressing Escherichia coli. PLoS ONE 6, e25409.

Hof, H. (1989). Antibacterial activities of the antiparasitic drugs nifurtimox and benznidazole. Antimicrob. Agents Chemother. 33, 404-405. 
Hoffman, R.M. (2011). Tumor-seeking Salmonella amino acid auxotrophs. Curr. Opin. Biotechnol. 22, 917-923.

Horvat, C.N. (2012). Development and Applications of Nitroreductase Activated Masked Fluorophores. PhD Thesis. School of Biological Sciences, Victoria University of Wellington, Wellington.

Immonen, A., Vapalahti, M., Tyynelä, K., Hurskainen, H., Sandmair, A., Vanninen, R., Langford, G., Murray, N., and Ylä-Herttuala, S. (2004). AdvHSV-tk gene therapy with intravenous ganciclovir improves survival in human malignant glioma: a randomised, controlled study. Mol. Ther. 10, 967-972.

Jaberipour, M., Vass, S.O., Guise, C.P., Grove, J.I., Knox, R.J., Hu, L., Hyde, E.I., and Searle, P.F. (2010). Testing double mutants of the enzyme nitroreductase for enhanced cell sensitisation to prodrugs: effects of combining beneficial single mutations. Biochem. Pharmacol. 79, 102-111.

Jacobs, A., Voges, J., Reszka, R., Lercher, M., Gossmann, A., Kracht, L., Kaestle, C., Wagner, R., Wienhard, K., and Heiss, W. (2001). Positron-emission tomography of vector-mediated gene expression in gene therapy for gliomas. The Lancet 358, 727-729.

Jameson, M.B., Rischin, D., Pegram, M., Gutheil, J., Patterson, A.V., Denny, W.A., and Wilson, W.R. (2010). A phase I trial of PR-104, a nitrogen mustard prodrug activated by both hypoxia and aldo-keto reductase 1C3, in patients with solid tumors. Cancer Chemother. Pharmacol. 65, 791-801.

Janssen, H.L.K., Hoebers, F.J., Sprong, D., Goethals, L., Williams, K.J., Stratford, I.J., Haustermans, K.M., Balm, A.J., and Begg, A.C. (2004). Differentiation-associated staining with anti-pimonidazole antibodies in head and neck tumors. Radiother. Oncol. 70, 91-97.

Jarrom, D., Jaberipour, M., Guise, C.P., Daff, S., White, S.A., Searle, P.F., and Hyde, E.I. (2009). Steady-State and Stopped-Flow Kinetic Studies of Three Escherichia coli NfsB Mutants with Enhanced Activity for the Prodrug CB1954. Biochemistry 48, 7665-7672.

Jawanda, N., Ebalunode, J., Gribenko, A., Briggs, J., Lee, J.C., and Tu, S.-C. (2008). A single-residue mutation destabilizes Vibrio harveyi flavin reductase FRP dimer. Arch. Biochem. Biophys. 472, 51-57.

Jenkins, T.C., Naylor, M.A., O’Neill, P., Threadgill, M.D., Cole, S., Stratford, I.J., Adams, G.E., Fielden, E.M., Suto, M.J., and Stier, M.A. (1990). Synthesis and evaluation of alpha-[[(2-haloethyl)amino]methyl]-2-nitro-1H-imidazole-1-ethanols as prodrugs of alpha-[(1-aziridinyl)methyl]-2-nitro-1H-imidazole-1-ethanol (RSU-1069) and its analogues which are radiosensitizers and bioreductively activated cytotoxins. J. Med. Chem. 33, 2603-2610.

Jerabek, P.A., Patrick, T.B., Kilbourn, M.R., Dischino, D.D., and Welch, M.J. (1986). Synthesis and biodistribution of 18F-labeled fluoronitroimidazoles: potential in vivo markers of hypoxic tissue. Int. J. Rad. Appl. Instrum. A 37, 599-605.

Johansson, E., Parkinson, G.N., Denny, W.A., and Neidle, S. (2003). Studies on the Nitroreductase Prodrug-Activating System. Crystal Structures of Complexes with the 
Inhibitor Dicoumarol and Dinitrobenzamide Prodrugs and of the Enzyme Active Form. J. Med. Chem. 46, 4009-4020.

Joseph, P., Jaiswal, A.K., Stobbe, C.C., and Chapman, J.D. (1994). The role of specific reductases in the intracellular activation and binding of 2-nitroimidazoles. Int. J. Radiat. Oncol. Biol. Phys. 29, 351-355.

Kasinskas, R.W., and Forbes, N.S. (2007). Salmonella typhimurium Lacking Ribose Chemoreceptors Localize in Tumor Quiescence and Induce Apoptosis. Cancer Res. 67, 3201-3209.

Kaufman, H.L., and Bines, S.D. (2010). OPTIM trial: a Phase III trial of an oncolytic herpes virus encoding GM-CSF for unresectable stage III or IV melanoma. Future Oncol. 6, 941-949.

Kaufman, H.L., Kim, D.W., DeRaffele, G., Mitcham, J., Coffin, R.S., and Kim-Schulze, S. (2010). Local and distant immunity induced by intralesional vaccination with an oncolytic herpes virus encoding GM-CSF in patients with stage IIIc and IV melanoma. Ann. Surg. Oncol. 17, 718-730.

Kelly, E., and Russell, S.J. (2007). History of Oncolytic Viruses: Genesis to Genetic Engineering. Mol. Ther. 15, 651-659.

Kelly, K.R., Espitia, C.M., Mahalingam, D., Oyajobi, B.O., Coffey, M., Giles, F.J., Carew, J.S., and Nawrocki, S.T. (2012). Reovirus therapy stimulates endoplasmic reticular stress, NOXA induction, and augments bortezomib-mediated apoptosis in multiple myeloma. Oncogene 31, 3023-3038.

Kennedy, A.S., Raleigh, J.A., Perez, G.M., Calkins, D.P., Thrall, D.E., Novotny, D.B., and Varia, M.A. (1997). Proliferation and hypoxia in human squamous cell carcinoma of the cervix: first report of combined immunohistochemical assays. Int. J. Radiat. Oncol. Biol. Phys. 37, 897-905.

Kim, K.H., Dmitriev, I., O’Malley, J.P., Wang, M., Saddekni, S., You, Z., Preuss, M.A., Harris, R.D., Aurigemma, R., Siegal, G.P., et al. (2012). A phase I clinical trial of Ad5.SSTR/TK.RGD, a novel infectivity-enhanced bicistronic adenovirus, in patients with recurrent gynecologic cancer. Clin. Cancer Res. 18, 3440-3451.

King, I., Itterson, M., and Bermudes, D. (2009). Tumor-Targeted Salmonella typhimurium Overexpressing Cytosine Deaminase: A Novel, Tumor-Selective Therapy. In Gene Therapy of Cancer, W. Walther, and U.S. Stein, eds. (Humana Press), pp. 649659.

Kirn, D.H., and Thorne, S.H. (2009). Targeted and armed oncolytic poxviruses: a novel multi-mechanistic therapeutic class for cancer. Nat. Rev. Cancer 9, 64-71.

Klutz, K., Willhauck, M.J., Wunderlich, N., Zach, C., Anton, M., SenekowitschSchmidtke, R., Göke, B., and Spitzweg, C. (2011). Sodium Iodide Symporter (NIS)Mediated Radionuclide $\left({ }^{131} \mathrm{I},{ }^{188} \mathrm{Re}\right)$ Therapy of Liver Cancer After Transcriptionally Targeted Intratumoral in vivo NIS Gene Delivery. Human Gene Ther. 22, 1403-1412. 
Knox, R.J., Boland, M.P., Friedlos, F., Coles, B., Southan, C., and Roberts, J.J. (1988). The nitroreductase enzyme in walker cells that activates 5-(aziridin-1-yl)-2,4dinitrobenzamide (CB 1954) to 5-(aziridin-1-YL)-4-hydroxylamino-2-nitrobenzamide is a form of $\mathrm{NAD}(\mathrm{P}) \mathrm{H}$ dehydrogenase (quinone) (EC 1.6.99.2). Biochem. Pharmacol. 37, 4671-4677.

Knox, R.J., Friedlos, F., Marchbank, T., and Roberts, J.J. (1991). Bioactivation of CB 1954: reaction of the active 4-hydroxylamino derivative with thioesters to form the ultimate DNA-DNA interstrand crosslinking species. Biochem. Pharmacol. 42, 16911697.

Kobori, T., Sasaki, H., Lee, W.C., Zenno, S., Saigo, K., Murphy, M.E., and Tanokura, M. (2001). Structure and site-directed mutagenesis of a flavoprotein from Escherichia coli that reduces nitrocompounds: alteration of pyridine nucleotide binding by a single amino acid substitution. J. Biol. Chem. 276, 2816-2823.

Koch, C.J., Scheuermann, J.S., Divgi, C., Judy, K.D., Kachur, A.V., Freifelder, R., Reddin, J.S., Karp, J., Stubbs, J.B., Hahn, S.M., et al. (2010). Biodistribution and dosimetry of 18F-EF5 in cancer patients with preliminary comparison of 18F-EF5 uptake versus EF5 binding in human glioblastoma. Eur. J. Nuc. Med. Mol. Imaging 37, 20482059.

Koder, R.L., and Miller, A.F. (1998). Steady-state kinetic mechanism, stereospecificity, substrate and inhibitor specificity of Enterobacter cloacae nitroreductase. Biochim. Biophys. Acta-Protein Struct. Molec. Enzym. 1387, 395-405.

Koder, R.L., Haynes, C.A., Rodgers, M.E., Rodgers, D.W., and Miller, A.-F. (2002). Flavin Thermodynamics Explain the Oxygen Insensitivity of Enteric Nitroreductases $\uparrow$. Biochemistry 41, 14197-14205.

Kogelnik, H.D., Meyer, J.H., Jentzsch, K., Szepesi, T., Kärcher, K.H., Maida, E., Mamoli, B., Wessely, P., and Zaunbauer, F. (1978). Further clinical experiences of a phase I study with the hypoxic cell radiosensitizer misonidazole. Br. J. Cancer Suppl. 3, 281-285.

Koike, H., Sasaki, H., Kobori, T., Zenno, S., Saigo, K., Murphy, M.E.., Adman, E.T., and Tanokura, M. (1998). 1.8 A crystal structure of the major NAD(P)H:FMN oxidoreductase of a bioluminescent bacterium, Vibrio fischeri: overall structure, cofactor and substrateanalog binding, and comparison with related flavoproteins. J. Mol. Biol. 280, 259-273.

Kokoris, M.S., and Black, M.E. (2002). Characterization of Herpes Simplex Virus type 1 thymidine kinase mutants engineered for improved ganciclovir or acyclovir activity. Protein Sci. 11, 2267-2272.

Kokoris, M.S., Sabo, P., Adman, E.T., and Black, M.E. (1999). Enhancement of tumor ablation by a selected HSV-1 thymidine kinase mutant. Gene Ther. 6, 1415-1426.

Komar, G., Seppänen, M., Eskola, O., Lindholm, P., Grönroos, T.J., Forsback, S., Sipilä, H., Evans, S.M., Solin, O., and Minn, H. (2008). 18F-EF5: a new PET tracer for imaging hypoxia in head and neck cancer. J. Nucl. Med. 49, 1944-1951. 
Kuehne, S.A., Heap, J.T., Cooksley, C.M., Cartman, S.T., and Minton, N.P. (2011). ClosTron-mediated engineering of Clostridium. Methods Mol. Biol. 765, 389-407.

Kumar, A., and Singh, S. (2012). Directed evolution: tailoring biocatalysts for industrial applications. Crit. Rev. Biotechnol.

Kuo, C., Coquoz, O., Troy, T.L., Xu, H., and Rice, B.W. (2007). Three-dimensional reconstruction of in vivo bioluminescent sources based on multispectral imaging. J. Biomed. Opt 12, 024007-024007.

Kwak, Y.H., Lee, D.S., and Kim, H.B. (2003). Vibrio harveyi nitroreductase is also a chromate reductase. Appl. Environ. Microbiol. 69, 4390-4395.

Leitsch, D., Kolarich, D., Wilson, I.B.H., Altmann, F., and Duchêne, M. (2007). Nitroimidazole action in Entamoeba histolytica: a central role for thioredoxin reductase. PLoS Biol. 5, e211.

Li, Z., and Conti, P.S. (2010). Radiopharmaceutical chemistry for positron emission tomography. Advanc. Drug Deliv. Rev. 62, 1031-1051.

Li, N., Zhou, J., Weng, D., Zhang, C., Li, L., Wang, B., Song, Y., He, Q., Lin, D., Chen, D., et al. (2007). Adjuvant Adenovirus-Mediated Delivery of Herpes Simplex Virus Thymidine Kinase Administration Improves Outcome of Liver Transplantation in Patients with Advanced Hepatocellular Carcinoma. Clin Cancer Res 13, 5847-5854.

Li, X., Fu, G.-F., Fan, Y.-R., Liu, W.-H., Liu, X.-J., Wang, J.-J., and Xu, G.-X. (2003). Bifidobacterium adolescentis as a delivery system of endostatin for cancer gene therapy: selective inhibitor of angiogenesis and hypoxic tumor growth. Cancer Gene. Ther. 10, $105-111$.

Lim, J.L., and Berridge, M.S. (1993). An efficient radiosynthesis of [18F]fluoromisonidazole. Appl. Radiat. Isot. 44, 1085-1091.

Liochev, S.I., Hausladen, A., and Fridovich, I. (1999). Nitroreductase A is regulated as a member of the soxRS regulon of Escherichia coli. PNAS 96, 3537.

Liu, G., Zhou, J., Lv, H., Xiang, X., Wang, J., Zhou, M., and Qv, Y. (2007). Azoreductase from Rhodobacter sphaeroides AS1.1737 is a flavodoxin that also functions as nitroreductase and flavin mononucleotide reductase. Appl. Microbiol. Biotechnol. 76, $1271-1279$.

Liu, G., Zhou, J., Jin, R., Zhou, M., Wang, J., Lu, H., and Qu, Y. (2008a). Enhancing survival of Escherichia coli by expression of azoreductase AZR possessing quinone reductase activity. Appl. Microbiol. Biotechnol. 80, 409-416.

Liu, M., Lei, B., Ding, Q., Lee, J.C., and Tu, S.-C. (1997). Vibrio harveyi NADPH:FMN Oxidoreductase: Preparation and Characterization of the Apoenzyme and MonomerDimer Equilibrium. Arch. Biochem. Biophys. 337, 89-95.

Liu, S.C., Minton, N.P., Giaccia, A.J., and Brown, J.M. (2002). Anticancer efficacy of systemically delivered anaerobic bacteria as gene therapy vectors targeting tumor hypoxia/necrosis. Gene Ther. 9, 291-296. 
Liu, S.-C., Ahn, G.-O., Kioi, M., Dorie, M.-J., Patterson, A.V., and Brown, J.M. (2008b). Optimized clostridium-directed enzyme prodrug therapy improves the antitumor activity of the novel DNA cross-linking agent PR-104. Cancer Res. 68, 7995-8003.

Loh, K.D., Gyaneshwar, P., Markenscoff Papadimitriou, E., Fong, R., Kim, K.-S., Parales, R., Zhou, Z., Inwood, W., and Kustu, S. (2006). A previously undescribed pathway for pyrimidine catabolism. PNAS. 103, 5114-5119.

Loon, J., Janssen, M.H.M., Öllers, M., Aerts, H.J.W.L., Dubois, L., Hochstenbag, M., Dingemans, A.-M.C., Lalisang, R., Brans, B., Windhorst, B., et al. (2010). PET imaging of hypoxia using [18F]HX4: a phase I trial. Eur J Nucl Med Mol Imaging 37, 1663-1668.

Lord, E.M., Harwell, L., and Koch, C.J. (1993). Detection of hypoxic cells by monoclonal antibody recognizing 2-nitroimidazole adducts. Cancer Res 53, 5721-5726.

Lovering, A.L., Hyde, E.I., Searle, P.F., and White, S.A. (2001). The structure of Escherichia coli nitroreductase complexed with nicotinic acid: three crystal forms at 1.7 $\AA$ A, $1.8 \AA$ and $2.4 \AA$ resolution. J. Mol. Biol. 309, 203-213.

Lu, M., Freytag, S.O., Stricker, H., Kim, J.H., Barton, K., and Movsas, B. (2011). Adaptive seamless design for an efficacy trial of replication-competent adenovirusmediated suicide gene therapy and radiation in newly-diagnosed prostate cancer (ReCAP Trial). Contemp. Clin. Trials 32, 453-460.

Luo, C., Wang, X., Long, J., and Liu, J. (2006). An NADH-tetrazolium-coupled sensitive assay for malate dehydrogenase in mitochondria and crude tissue homogenates. $J$. Biochem. Biophys.Methods 68, 101-111.

Ma, J., and Waxman, D.J. (2007). Collaboration between Hepatic and Intratumoral Prodrug Activation in a P450 Prodrug-activation Gene Therapy Model for Cancer Treatment. Mol. Cancer Ther. 6, 2879-2890.

Mahy, P., Geets, X., Lonneux, M., Levêque, P., Christian, N., De Bast, M., Gillart, J., Labar, D., Lee, J., and Grégoire, V. (2008). Determination of tumour hypoxia with [18F]EF3 in patients with head and neck tumours: a phase I study to assess the tracer pharmacokinetics, biodistribution and metabolism. Eur. J. Nucl. Med. Mol. Imaging 35, $1282-1289$.

Mallia, M.B., Mathur, A., Subramanian, S., Banerjee, S., Sarma, H.D., and Venkatesh, M. (2005). A novel [99mTc[triple bond] $\mathrm{N}] 2+$ complex of metronidazole xanthate as a potential agent for targeting hypoxia. Bioorg. Med. Chem. Lett. 15, 3398-3401.

Marcu, L.G., Bezak, E., and Filip, S.M. (2012). The role of PET imaging in overcoming radiobiological challenges in the treatment of advanced head and neck cancer. Cancer Treat. Rev. 38, 185-193.

Mattes, W.B., Hartley, J.A. and Kohn, K.W. (1986). DNA sequence selectivity of guanine-N7 alkylation by nitrogen mustards. Nucleic Acids Res. 14, 2971-2987.

Mayer, K.M., and Arnold, F.H. (2002). A colorimetric assay to quantify dehydrogenase activity in crude cell lysates. J. Biomol. Screen 7, 135-140. 
McCart, J.A., Mehta, N., Scollard, D., Reilly, R.M., Carrasquillo, J.A., Tang, N., Deng, H., Miller, M., Xu, H., Libutti, S.K., et al. (2004). Oncolytic Vaccinia Virus Expressing the Human Somatostatin Receptor SSTR2: Molecular Imaging after Systemic Delivery Using 111In-Pentetreotide. Mol.Ther. 10, 553-561.

McCormack, E., Silden, E., West, R.M., Pavlin, T., Micklem, D.R., Lorens, J., Haug, B.E., Cooper, M.E., and Gjertsen, B.T. (2012). Nitroreductase, a near infrared reporter platform for in vivo time-domain optical imaging of metastatic cancer. Cancer Res.

McKeage, M.J., Gu, Y., Wilson, W.R., Hill, A., Amies, K., Melink, T.J., and Jameson, M.B. (2011). A phase I trial of PR-104, a pre-prodrug of the bioreductive prodrug PR104A, given weekly to solid tumour patients. BMC Cancer 11, 432.

Mees, G., Dierckx, R., Vangestel, C., and Wiele, C. (2009). Molecular imaging of hypoxia with radiolabelled agents. Eur. J. Nucl. Med. Mol. Imaging 36, 1674-1686.

Mesnil, M., and Yamasaki, H. (2000). Bystander Effect in Herpes Simplex VirusThymidine Kinase/Ganciclovir Cancer Gene Therapy: Role of Gap-junctional Intercellular Communication1. Cancer Res. 60, 3989-3999.

Miller, J.H. (1972). Experiments in molecular genetics (Cold Spring Harbor Laboratory Press).

Ministry of Health. (2012). Cancer: New Registrations and Deaths 2009. Wellington: Ministry of Health.

Min, J.J. and Gambhir, S.S. (2008). Molecular imaging of PET reporter gene expression. Handb. Exp. Pharmacol. 185 Pt2, 277-303.

Mitchell, D.J., and Minchin, R.F. (2008). E. coli nitroreductase/CB1954 gene-directed enzyme prodrug therapy: role of arylamine $\mathrm{N}$-acetlytransferase 2. Cancer Gene Ther. 15, 758-764.

Moch, C., Schrögel, O., and Allmansberger, R. (2000). Transcription of the nfrA-ywcH operon from Bacillus subtilis is specifically induced in response to heat. J. Bacteriol. 182, 4384-4393.

Moolten, F.L. (1986). Tumor chemosensitivity conferred by inserted herpes thymidine kinase genes: paradigm for a prospective cancer control strategy. Cancer Res. 46, 52765281.

Morokutti, A., Lyskowski, A., Sollner, S., Pointner, E., Fitzpatrick, T.B., Kratky, C., Gruber, K., and Macheroux, P. (2005). Structure and function of YcnD from Bacillus subtilis, a flavin-containing oxidoreductase. Biochemistry 44, 13724-13733.

Morrissey, D., O'Sullivan, G.C., and Tangney, M. (2010). Tumour targeting with systemically administered bacteria. Curr. Gene Ther. 10, 3-14.

Mortensen, L.S., Johansen, J., Kallehauge, J., Primdahl, H., Busk, M., Lassen, P., Alsner, J., Sørensen, B.S., Toustrup, K., Jakobsen, S., et al. (2012). FAZA PET/CT hypoxia imaging in patients with squamous cell carcinoma of the head and neck treated with radiotherapy: results from the DAHANCA 24 trial. Radiother. Oncol. 105, 14-20. 
Mowday, A.M. (2010). The evaluation of bacterial nitroreductases for gene therapy. Masters Thesis. Auckland Cancer Society Research Centre. Faculty of Medical and Health Sciences. University of Auckland. Auckland.

Msaouel, P., Dispenzieri, A., and Galanis, E. (2009a). Clinical testing of engineered oncolytic measles virus strains in the treatment of cancer: An overview. Curr. Opin. Mol. Ther. $11,43$.

Msaouel, P., Iankov, I.D., Allen, C., Aderca, I., Federspiel, M.J., Tindall, D.J., Morris, J.C., Koutsilieris, M., Russell, S.J., and Galanis, E. (2009b). Noninvasive Imaging and Radiovirotherapy of Prostate Cancer Using an Oncolytic Measles Virus Expressing the Sodium Iodide Symporter. Mol. Ther. 17, 2041-2048.

Nakanishi, M., Yatome, C., Ishida, N., and Kitade, Y. (2001). Putative ACP Phosphodiesterase Gene (acpD) Encodes an Azoreductase. J. Biol. Chem. 276, 4639446399.

Nasu, Y., Saika, T., Ebara, S., Kusaka, N., Kaku, H., Abarzua, F., Manabe, D., Thompson, T.C., and Kumon, H. (2007). Suicide gene therapy with adenoviral delivery of HSV-tK gene for patients with local recurrence of prostate cancer after hormonal therapy. Mol. Ther. 15, 834-840.

Niesen, F.H., Berglund, H., and Vedadi, M. (2007). The use of differential scanning fluorimetry to detect ligand interactions that promote protein stability. Nat. Protocols 2, 2212-2221.

Nokhbeh, M.R., Boroumandi, S., Pokorny, N., Koziarz, P., Paterson, E.S., and Lambert, I.B. (2002). Identification and characterization of SnrA, an inducible oxygen-insensitive nitroreductase in Salmonella enterica serovar Typhimurium TA1535. Mutat. Res. 508, $59-70$.

Nolting, D., C. Gore, J., and Pham, W. (2011). Near-Infrared Dyes: Probe Development and Applications in Optical Molecular Imaging. Curr. Org. Synth. 8, 521-534.

Norman, A., Hestbjerg Hansen, L., and Sorensen, S.J. (2005). Construction of a ColD cda Promoter-Based SOS-Green Fluorescent Protein Whole-Cell Biosensor with Higher Sensitivity toward Genotoxic Compounds than Constructs Based on recA, umuDC, or sulA Promoters. Appl. Environ. Microbiol. 71, 2338-2346.

Olekhnovich, I.N., Goodwin, A., and Hoffman, P.S. (2009). Characterization of the $\mathrm{NAD}(\mathrm{P}) \mathrm{H}$ oxidase and metronidazole reductase activities of the RdxA nitroreductase of Helicobacter pylori. FEBS Journal 276, 3354-3364.

Olive, P.L. (1979). Correlation between Metabolic Reduction Rates and Electron Affinity of Nitroheterocycles. Cancer Res 39, 4512-4515.

Orna, M.V., and Mason, R.P. (1989). Correlation of kinetic parameters of nitroreductase enzymes with redox properties of nitroaromatic compounds. J. Biol. Chem. 264, 1237912384.

Padhani, A. (2006). PET imaging of tumour hypoxia. Cancer Imaging 6, S117-S121. 
Palmer, D.H., Mautner, V., Mirza, D., Oliff, S., Gerritsen, W., Van der Sijp, J.R.M., Hubscher, S., Reynolds, G., Bonney, S., Rajaratnam, R., et al. (2004). Virus-directed enzyme prodrug therapy: intratumoral administration of a replication-deficient adenovirus encoding nitroreductase to patients with resectable liver cancer. J. Clin. Oncol. 22, 15461552.

Parato, K.A., Breitbach, C.J., Le Boeuf, F., Wang, J., Storbeck, C., Ilkow, C., Diallo, J.S., Falls, T., Burns, J., Garcia, V., et al. (2011). The Oncolytic Poxvirus JX-594 Selectively Replicates in and Destroys Cancer Cells Driven by Genetic Pathways Commonly Activated in Cancers. Mol. Ther. 20, 749-758.

Park, B.-H., Hwang, T., Liu, T.-C., Sze, D.Y., Kim, J.-S., Kwon, H.-C., Oh, S.Y., Han, S.-Y., Yoon, J.-H., Hong, S.-H., et al. (2008). Use of a targeted oncolytic poxvirus, JX594, in patients with refractory primary or metastatic liver cancer: a phase I trial. Lancet Oncol. 9, 533-542.

Parkinson, G.N., Skelly, J.V., and Neidle, S. (2000). Crystal Structure of FMNDependent Nitroreductase from Escherichia coli B: A Prodrug-Activating Enzyme. J. Med. Chem. 43, 3624-3631.

Patel, P., Young, J.G., Mautner, V., Ashdown, D., Bonney, S., Pineda, R.G., Collins, S.I., Searle, P.F., Hull, D., Peers, E., et al. (2009). A phase I/II clinical trial in localized prostate cancer of an adenovirus expressing nitroreductase with CB1954 [correction of CB1984]. Mol. Ther. 17, 1292-1299.

Patridge, E.V., and Ferry, J.G. (2006). WrbA from Escherichia coli and Archaeoglobus fulgidus Is an NAD(P)H:Quinone Oxidoreductase. J. Bacteriol. 188, 3498-3506.

Penheiter, A., J. Russell, S., and K. Carlson, S. (2012). The Sodium Iodide Symporter (NIS) as an Imaging Reporter for Gene, Viral, and Cell-based Therapies. Curr. Gene Ther. 12, 33-47.

Pennington, J.M., and Rosenberg, S.M. (2007). Spontaneous DNA breakage in single living Escherichia coli cells. Nat. Gen. 39, 797-802.

Peñuelas, I., Haberkorn, U., Yaghoubi, S., and Gambhir, S.S. (2005a). Gene therapy imaging in patients for oncological applications. Eur. J. Nucl. Med. Mol. Imaging 32 Suppl 2, S384-403.

Peñuelas, I., Mazzolini, G., Boán, J.F., Sangro, B., Martí-Climent, J., Ruiz, M., Ruiz, J., Satyamurthy, N., Qian, C., Barrio, J.R., et al. (2005b). Positron Emission Tomography Imaging of Adenoviral-Mediated Transgene Expression in Liver Cancer Patients. Gastroenterol. 128, 1787-1795.

Peterson, F.J., Mason, R.P., Hovsepian, J., and Holtzman, J.L. (1979). Oxygen-sensitive and -insensitive nitroreduction by Escherichia coli and rat hepatic microsomes. J. Biol. Chem. 254, 4009-4014.

Ponomarev, V. (2009). Nuclear imaging of cancer cell therapies. J. Nucl. Med. 50, 10131016. 
Prosser, G.A. (2011). Discovery and Optimisation of Bacterial Nitroreductases for use in Anti-cancer Gene Therapy. PhD Thesis. School of Biological Sciences, Victoria University of Wellington, Wellington.

Prosser, G.A., Copp, J.N., Syddall, S.P., Williams, E.M., Smaill, J.B., Wilson, W.R., Patterson, A.V., and Ackerley, D.F. (2010a). Discovery and evaluation of Escherichia coli nitroreductases that activate the anti-cancer prodrug CB1954. Biochem. Pharmacol. 79, 678-687.

Prosser, G.A., Copp, J.N., Mowday, A.M., Guise, C.P., Syddall, S.P., Williams, E.M., Horvat, C.N., Swe, P.M., Ashoorzadeh, A., Denny, W.A., et al. (2013). Creation and screening of a multi-family bacterial oxidoreductase library to discover novel nitroreductases that efficiently activate the bioreductive prodrugs CB1954 and PR-104A. Biochem. Pharmacol. 85, 1091-1103.

Quillardet, P., Huisman, O., D’Ari, R., and Hofnung, M. (1982). SOS chromotest, a direct assay of induction of an SOS function in Escherichia coli K-12 to measure genotoxicity. PNAS 79, 5971-5975.

Race, P.R., Lovering, A.L., Green, R.M., Ossor, A., White, S.A., Searle, P.F., Wrighton, C.J., and Hyde, E.I. (2005). Structural and Mechanistic Studies of Escherichia coli Nitroreductase with the Antibiotic Nitrofurazone. J. Biol. Chem. 280, 13256 -13264.

Rahmim, A., and Zaidi, H. (2008). PET versus SPECT: strengths, limitations and challenges. Nucl. Med. Commun. 29, 193-207.

Rainov, N.G. (2000). A Phase III Clinical Evaluation of Herpes Simplex Virus Type 1 Thymidine Kinase and Ganciclovir Gene Therapy as an Adjuvant to Surgical Resection and Radiation in Adults with Previously Untreated Glioblastoma Multiforme. Hum. Gene Ther. 11, 2389-2401.

Rajecki, M., Kangasmäki, A., Laasonen, L., Escutenaire, S., Hakkarainen, T., Haukka, J., Ristimäki, A., Kairemo, K., Kangasniemi, L., Kiljunen, T., et al. (2011). Sodium iodide symporter SPECT imaging of a patient treated with oncolytic adenovirus Ad5/3- $\Delta 24$ hNIS. Mol. Ther. 19, 629-631.

Rajendran, J.G., Schwartz, D.L., O’Sullivan, J., Peterson, L.M., Ng, P., Scharnhorst, J., Grierson, J.R., and Krohn, K.A. (2006). Tumor Hypoxia Imaging with [F-18] Fluoromisonidazole Positron Emission Tomography in Head and Neck Cancer. Clin. Cancer Res. 12, 5435-5441.

Rao, G., Lee, J.-K., and Zhao, H. (2013). Directed evolution of phloroglucinol synthase PhlD with increased stability for phloroglucinol production. Appl. Microbiol. Biotechnol. 97, 1-7.

Rasey, J.S., Grunbaum, Z., Magee, S., Nelson, N.J., Olive, P.L., Durand, R.E., and Krohn, K.A. (1987). Characterization of radiolabeled fluoromisonidazole as a probe for hypoxic cells. Radiat. Res. 111, 292-304.

Rasey, J.S., Hofstrand, P.D., Chin, L.K., and Tewson, T.J. (1999). Characterization of [18F]fluoroetanidazole, a new radiopharmaceutical for detecting tumor hypoxia. J. Nucl. Med. 40, 1072-1079. 
Razgulin, A., Ma, N., and Rao, J. (2011). Strategies for in vivo imaging of enzyme activity: an overview and recent advances. Chem. Soc. Rev. 40, 4186-4216.

Reetz, M.T., and Carballeira, J.D. (2007). Iterative saturation mutagenesis (ISM) for rapid directed evolution of functional enzymes. Nat. Protoc. 2, 891-903.

Reetz, M.T., Carballeira, J.D., Peyralans, J., Höbenreich, H., Maichele, A., and Vogel, A. (2006). Expanding the substrate scope of enzymes: combining mutations obtained by CASTing. Chemistry 12, 6031-6038.

Rischin, D., Hicks, R.J., Fisher, R., Binns, D., Corry, J., Porceddu, S., and Peters, L.J. (2006). Prognostic significance of [18F]-misonidazole positron emission tomographydetected tumor hypoxia in patients with advanced head and neck cancer randomly assigned to chemoradiation with or without tirapazamine: a substudy of Trans-Tasman Radiation Oncology Group Study 98.02. J. Clin. Oncol. 24, 2098-2104.

Robins, K., Hooks, D., Rehm, B., and Ackerley, D.F. Escherichia coli NemA is an efficient chromate reductase that can be biologically immobilized to provide a cell free system for remediation of hexavalent chromium. PLoS ONE 8, e59200.

Rojas, J.J., and Thorne, S.H. (2012). Theranostic Potential of Oncolytic Vaccinia Virus. Theranostics 2, 363-373.

Roldán, M.D., Pérez-Reinado, E., Castillo, F., and Moreno-Vivián, C. (2008). Reduction of polynitroaromatic compounds: the bacterial nitroreductases. FEMS Microbiol. Rev. 32, 474-500.

Russell, S.J., Peng, K.-W., and Bell, J.C. (2012). Oncolytic virotherapy. Nat. Biotechnol. 30, 658-670.

Sambrook, J., and Russell, D.W. (2001). Molecular Cloning: A Laboratory Manual (Cold Spring Harbor Laboratory Press).

Sangro, B., Mazzolini, G., Ruiz, M., Ruiz, J., Quiroga, J., Herrero, I., Qian, C., Benito, A., Larrache, J., Olaguie, C., et al. (2010). A phase I clinical trial of thymidine kinasebased gene therapy in advanced hepatocellular carcinoma. Cancer Gene Ther. 17, 837843.

Schmidt, C. (2011). Amgen spikes interest in live virus vaccines for hard-to-treat cancers. Nat. Biotechnol. 29, 295-296.

Sekar, T.V., Foygel, K., Willmann, J.K., and Paulmurugan, R. (2012). Dual-therapeutic reporter genes fusion for enhanced cancer gene therapy and imaging. Gene Ther. 20, 529537.

Senzer, N.N., Kaufman, H.L., Amatruda, T., Nemunaitis, M., Reid, T., Daniels, G., Gonzalez, R., Glaspy, J., Whitman, E., Harrington, K., et al. (2009). Phase II clinical trial of a granulocyte-macrophage colony-stimulating factor-encoding, second-generation oncolytic herpesvirus in patients with unresectable metastatic melanoma. J. Clin. Oncol. 27, 5763-5771. 
Shapiro, E., and Baneyx, F. (2002). Stress-based identification and classification of antibacterial agents: second-generation Escherichia coli reporter strains and optimization of detection. Antimicrob. Agents Chemother. 46, 2490-2497.

Shestopalov, A.I., Bogachev, A.V., Murtazina, R.A., Viryasov, M.B., and Skulachev, V.P. (1997). Aeration-dependent changes in composition of the quinone pool in Escherichia coli: Evidence of post-transcriptional regulation of the quinone biosynthesis. FEBS Letters 404, 272-274.

Singleton, D.C., Li, D., Bai, S.Y., Syddall, S.P., Smaill, J.B., Shen, Y., Denny, W.A., Wilson, W.R., and Patterson, A.V. (2007). The nitroreductase prodrug SN 28343 enhances the potency of systemically administered armed oncolytic adenovirus ONYX411NTR. Cancer Gene Ther. 14, 953-967.

Sirsi, S.R. (2012). Advances in Ultrasound Mediated Gene Therapy Using Microbubble Contrast Agents. Theranostics 2, 1208-1222.

Soghomonyan, S.A., Doubrovin, M., Pike, J., Luo, X., Ittensohn, M., Runyan, J.D., Balatoni, J., Finn, R., Tjuvajev, J.G., Blasberg, R., et al. (2005). Positron emission tomography (PET) imaging of tumor-localized Salmonella expressing HSV1-TK. Cancer Gene Ther. 12, 101-108.

Spitzweg, C., Harrington, K.J., Pinke, L.A., Vile, R.G., and Morris, J.C. (2001). The Sodium Iodide Symporter and Its Potential Role in Cancer Therapy. JCEM 86, 33273335 .

Stojdl, D.F., Lichty, B., Knowles, S., Marius, R., Atkins, H., Sonenberg, N., and Bell, J.C. (2000). Exploiting tumor-specific defects in the interferon pathway with a previously unknown oncolytic virus. Nat. Med. 6, 821-825.

Strong, J.E., Coffey, M.C., Tang, D., Sabinin, P., and Lee, P.W. (1998). The molecular basis of viral oncolysis: usurpation of the Ras signaling pathway by reovirus. EMBO J 17, 3351-3362.

Swe, P.M., Copp, J.N., Green, L.K., Guise, C.P., Mowday, A.M., Smaill, J.B., Patterson, A.V., and Ackerley, D.F. (2012). Targeted mutagenesis of the Vibrio fischeri flavin reductase FRase I to improve activation of the anticancer prodrug CB1954. Biochem. Pharmacol. 84, 775-783.

Syddall, S. (2009). Novel Bacterial Nitroreductases and Bioreductive Substrates for Gene Therapy Applications (Molecular Medicine and Pathology - University of Auckland).

Tanner, J.J., Lei, B., Tu, S.C., and Krause, K.L. (1996). Flavin reductase P: structure of a dimeric enzyme that reduces flavin. Biochemistry 35, 13531-13539.

Theys, J., Pennington, O., Dubois, L., Anlezark, G., Vaughan, T., Mengesha, A., Landuyt, W., Anné, J., Burke, P.J., Dûrre, P., et al. (2006). Repeated cycles of Clostridium-directed enzyme prodrug therapy result in sustained antitumour effects in vivo. Br. J. Cancer 95, 1212-1219.

Thorne, S.H., Hwang, T.-H.H., O’Gorman, W.E., Bartlett, D.L., Sei, S., Kanji, F., Brown, C., Werier, J., Cho, J.-H., Lee, D.-E., et al. (2007). Rational strain selection and 
engineering creates a broad-spectrum, systemically effective oncolytic poxvirus, JX-963. J. Clin. Invest. 117, 3350-3358.

Thorne, S.H., Barak, Y., Liang, W., Bachmann, M.H., Rao, J., Contag, C.H., and Matin, A. (2009). CNOB/ChrR6, a new prodrug enzyme cancer chemotherapy. Mol. Cancer Ther. 8, 333-341.

Thorwarth, D., Eschmann, S.-M., Scheiderbauer, J., Paulsen, F., and Alber, M. (2005). Kinetic analysis of dynamic 18F-fluoromisonidazole PET correlates with radiation treatment outcome in head-and-neck cancer. BMC Cancer 5, 152.

Tjuvajev, J.G., Finn, R., Watanabe, K., Joshi, R., Oku, T., Kennedy, J., Beattie, B., Koutcher, J., Larson, S., and Blasberg, R.G. (1996). Noninvasive imaging of herpes virus thymidine kinase gene transfer and expression: a potential method for monitoring clinical gene therapy. Cancer Res. 56, 4087-4095.

Tjuvajev, J.G., Joshi, A., Callegari, J., Lindsley, L., Joshi, R., Balatoni, J., Finn, R., Larson, S.M., Sadelain, M., and Blasberg, R.G. (1999). A general approach to the noninvasive imaging of transgenes using cis-linked herpes simplex virus thymidine kinase. Neoplasia 1, 315-320.

Tocher, J.H. (1997). Reductive activation of nitroheterocyclic compounds. Gen. Pharmacol: Vascul. System 28, 485-487.

Toso, J.F., Gill, V.J., Hwu, P., Marincola, F.M., Restifo, N.P., Schwartzentruber, D.J., Sherry, R.M., Topalian, S.L., Yang, J.C., Stock, F., et al. (2002). Phase I study of the intravenous administration of attenuated Salmonella typhimurium to patients with metastatic melanoma. J. Clin. Oncol. 20, 142-152.

Trosko, J.E. (2003). The role of stem cells and gap junctional intercellular communication in carcinogenesis. J. Biochem. Mol. Biol. 36, 43-48.

Umer, B., Good, D., Anné, J., Duan, W., and Wei, M.Q. (2012). Clostridial Spores for Cancer Therapy: Targeting Solid Tumour Microenvironment. J. Toxicol. 2012, 1-8.

Vaara, M. (1992). Agents that increase the permeability of the outer membrane. Microbiol. Rev. 56, 395-411.

Varghese, A.J., and Whitmore, G.F. (1980). Binding to Cellular Macromolecules as a Possible Mechanism for the Cytotoxicity of Misonidazole. Cancer Res 40, 2165-2169.

Varia, M.A., Calkins-Adams, D.P., Rinker, L.H., Kennedy, A.S., Novotny, D.B., Fowler Jr., W.C., and Raleigh, J.A. (1998). Pimonidazole: A Novel Hypoxia Marker for Complementary Study of Tumor Hypoxia and Cell Proliferation in Cervical Carcinoma. Gynecol. Oncol. 71, 270-277.

Vass, S.O., Jarrom, D., Wilson, W.R., Hyde, E.I., and Searle, P.F. (2009). E. coli NfsA: an alternative nitroreductase for prodrug activation gene therapy in combination with CB1954. Br. J. Cancer 100, 1903-1911. 
Vaupel, P., Kallinowski, F., and Okunieff, P. (1989). Blood Flow, Oxygen and Nutrient Supply, and Metabolic Microenvironment of Human Tumors: A Review. Cancer Res. 49, 6449-6465.

Waerzeggers, Y., Monfared, P., Viel, T., Winkeler, A., Voges, J., and Jacobs, A.H. (2009). Methods to monitor gene therapy with molecular imaging. Methods 48, 146-160.

Wang, J., Foehrenbacher, A., Su, J., Patel, R., Hay, M.P., Hicks, K.O., and Wilson, W.R. (2012). The 2-Nitroimidazole EF5 Is a Biomarker for Oxidoreductases That Activate the Bioreductive Prodrug CEN-209 under Hypoxia. Clin. Cancer Res. 18, 1684-1695.

Wang, Y., Iyer, M., Annala, A.J., Chappell, S., Mauro, V., and Gambhir, S.S. (2005). Noninvasive Monitoring of Target Gene Expression by Imaging Reporter Gene Expression in Living Animals Using Improved Bicistronic Vectors. J. Nucl. Med. 46, $667-674$.

Wardman (2001). Electron Transfer and Oxidative Stress as Key Factors in the Design of Drugs Selectively Active in Hypoxia. Curr. Med. Chem. 8, 739-761.

Wardman, P. (1985). Some reactions and properties of nitro radical-anions important in biology and medicine. Environ. Health Perspect. 64, 309.

Wardman, P. (2007). Chemical Radiosensitizers for Use in Radiotherapy. Clin. Oncol.19, $397-417$.

Watanabe, M., Nishino, T., Takio, K., Sofuni, T., and Nohmi, T. (1998). Purification and characterization of wild-type and mutant "classical" nitroreductases of Salmonella typhimurium. L33R mutation greatly diminishes binding of FMN to the nitroreductase of S. typhimurium. J. Biol. Chem. 273, 23922-23928.

Weibel, S., Stritzker, J., Eck, M., Goebel, W., and Szalay, A.A. (2008). Colonization of experimental murine breast tumours by Escherichia coli K-12 significantly alters the tumour microenvironment. Cell. Microbiol. 10, 1235-1248.

Whitmore, G.F., Varghese, A.J., Gulyas, S. (1986). Reaction of 2-nitroimidazole metabolites with guanine and possible biological consequences. IARC Sci. Publ.70, 185196.

Williams, R.E., Rathbone, D.A., Scrutton, N.S., and Bruce, N.C. (2004). Biotransformation of Explosives by the Old Yellow Enzyme Family of Flavoproteins. Appl. Environ. Microbiol. 70, 3566-3574.

Wilson, W.R., Pullen, S.M., Hogg, A., Helsby, N.A., Hicks, K.O., and Denny, W.A. (2002). Quantitation of Bystander Effects in Nitroreductase Suicide Gene Therapy Using Three-Dimensional Cell Cultures. Cancer Res. 62, 1425-1432.

Yaghoubi, S.S., and Gambhir, S.S. (2007). PET imaging of herpes simplex virus type 1 thymidine kinase (HSV1-tk) or mutant HSV1-sr39tk reporter gene expression in mice and humans using [18F]FHBG. Nat. Prot. 1, 3069-3074. 
Yaghoubi, S.S., Jensen, M.C., Satyamurthy, N., Budhiraja, S., Paik, D., Czernin, J., and Gambhir, S.S. (2009). Noninvasive detection of therapeutic cytolytic T cells with 18FFHBG PET in a patient with glioma. Nat. Clin. Pract. Oncol. 6, 53-58.

Yanto, Y., Hall, M., and Bommarius, A.S. (2010). Nitroreductase from Salmonella typhimurium: characterization and catalytic activity. Org. Biomol. Chem. 8, 1826-1832.

Yu, Y.A., Shabahang, S., Timiryasova, T.M., Zhang, Q., Beltz, R., Gentschev, I., Goebel, W., and Szalay, A.A. (2004). Visualization of tumors and metastases in live animals with bacteria and vaccinia virus encoding light-emitting proteins. Nat. Biotechnol. 22, 313320.

Yu, Y.A., Zhang, Q., and Szalay, A.A. (2008). Establishment and characterization of conditions required for tumor colonization by intravenously delivered bacteria. Biotechnol. Bioeng. 100, 567-578.

Yuin, P., Esterby, M., and Van Dam, R.M. (2012). Emerging Technologies for Decentralized Production of PET Tracers. In Positron Emission Tomography - Current Clinical and Research Aspects, C.H. Hsieh, ed. (InTech), DOI: 10.5772/31390.

Zar,F.A., Bakkanagari, S.R., Moorthi, K.M.L.S.T., and Davis, M.B. (2007). A comparison of vancomycin and metronidazole for the treatment of Clostridium difficileassociated diarrhea, stratified by disease severity. Clin. Infect. Dis. 45, 302-307.

Zenno, S., Saigo, K., Kanoh, H., and Inouye, S. (1994). Identification of the gene encoding the major $\mathrm{NAD}(\mathrm{P}) \mathrm{H}$-flavin oxidoreductase of the bioluminescent bacterium Vibrio fischeri ATCC 7744. J.Bacteriol. 176, 3536.

Zenno, S., Koike, H., Kumar, A.N., Jayaraman, R., Tanokura, M., and Saigo, K. (1996a). Biochemical characterization of NfsA, the Escherichia coli major nitroreductase exhibiting a high amino acid sequence homology to Frp, a Vibrio harveyi flavin oxidoreductase. J. Bacteriol. 178, 4508-4514.

Zenno, S., Koike, H., Tanokura, M., and Saigo, K. (1996b). Conversion of NfsB, a minor Escherichia coli nitroreductase, to a flavin reductase similar in biochemical properties to FRase I, the major flavin reductase in Vibrio fischeri, by a single amino acid substitution. J. Bacteriol. 178, 4731-4733.

Zenno, S., Kobori, T., Tanokura, M., and Saigo, K. (1998). Conversion of NfsA, the major Escherichia coli nitroreductase, to a flavin reductase with an activity similar to that of Frp, a flavin reductase in Vibrio harveyi, by a single amino acid substitution. J. Bacteriol. 180, 422-425.

Zhang, Q., Yu, Y.A., Wang, E., Chen, N., Danner, R.L., Munson, P.J., Marincola, F.M., and Szalay, A.A. (2007). Eradication of Solid Human Breast Tumors in Nude Mice with an Intravenously Injected Light-Emitting Oncolytic Vaccinia Virus. Cancer Res. 67, 10038-10046.

Zhao, M., Yang, M., Li, X.-M., Jiang, P., Baranov, E., Li, S., Xu, M., Penman, S., and Hoffman, R.M. (2005). Tumor-targeting bacterial therapy with amino acid auxotrophs of GFP-expressing Salmonella typhimurium. PNAS 102, 755-760. 
Zhao, M., Geller, J., Ma, H., Yang, M., Penman, S., and Hoffman, R.M. (2007). Monotherapy with a tumor-targeting mutant of Salmonella typhimurium cures orthotopic metastatic mouse models of human prostate cancer. PNAS 104, 10170-10174.

Zurkiya, O., Chan, A.W.S., and Hu, X. (2008). MagA is sufficient for producing magnetic nanoparticles in mammalian cells, making it an MRI reporter. Mag. Reson. Med. 59, 1225-1231. 